\title{
Convex Optimisation for \\ Communication Systems
}

\author{
Sudhir Singh \\ B.E.(Hons), University of Wollongong
}

February 2015

A thesis submitted for the Degree of Doctor of Philosophy of Victoria University of Wellington

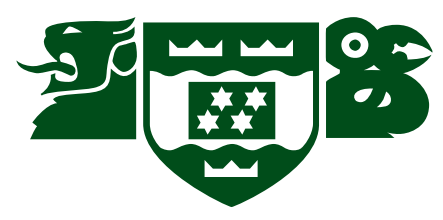

Faculty of Engineering

School of Engineering and Computer Science

Victoria University of Wellington 



\section{Declaration}

The contents of this thesis are the results of original research and have not been submitted for a higher degree to any other university or institution.

Much of the work in this thesis has been published as journal papers or peer reviewed conference proceedings. Following is a list of these papers.

1. S. Singh, P. D. Teal, P. A. Dmochowski, and A. J. Coulson, "Power allocation in underlay cognitive radio systems with feasibility detection," In Proc. Australian Communications Theory Workshop (AusCTW), pages 135-139, Feb. 2012 .

2. S. Singh, P. D. Teal, P. A. Dmochowski, and A. J. Coulson, "Interference management in cognitive radio systems - A convex optimisation approach," In Proc. IEEE Int. Conf. Commun. (ICC'12), pages 1884-1889, Jun. 2012.

3. S. Singh, P. D. Teal, P. A. Dmochowski, and A. J. Coulson, "Statistically robust cognitive radio beamforming," In Proc. Australian Communications Theory Workshop (AusCTW), pages 128-133, Feb. 2013.

4. S. Singh, P. D. Teal, P. A. Dmochowski, and A. J. Coulson, "Statistically robust cooperative beamforming for cognitive radio networks," In Proc. IEEE Int. Conf. Commun. (ICC'13), pages 2727-2732, Jun. 2013.

5. S. Singh, P. D. Teal, P. A. Dmochowski, and A. J. Coulson, "Interference Management in Cognitive Radio Systems With Feasibility Detection," IEEE Trans. Veh. Technol., 62(8):3711-3720, Oct. 2013.

6. S. Singh, P. D. Teal, P. A. Dmochowski, and A. J. Coulson, "Robust Cognitive Radio Cooperative Beamforming," IEEE Trans. Wireless Commun., 13(11):6370-6381, Nov. 2014. 
The research represented in this thesis has been performed jointly with Dr Paul D. Teal, Dr Pawel A. Dmochowski, and Dr Alan J. Coulson. The substantial majority of this work is my own.

Sudhir Singh

Victoria University of Wellington

February 2015 


\section{Acknowledgements}

I would like to take this opportunity to thank all the people who have contributed to this thesis.

I am grateful to Alan Coulson for giving me the opportunity to undertake this project and whose research programme formed the basis for this thesis. I am grateful to the management of Callaghan Innovation for supporting this project. I would also like to thank my supervisors, Paul Teal and Pawel Dmochowski who have helped shape the research direction.

My cat Billi for patiently sitting beside me as this thesis was written. Finally, and most importantly, my partner Sunita for always encouraging and supporting me. 



\section{Abstract}

In this thesis new robust methods for the efficient sharing of the radio spectrum for underlay cognitive radio (CR) systems are developed. These methods provide robustness against uncertainties in the channel state information (CSI) that is available to the cognitive radios. A stochastic approach is taken and the robust spectrum sharing methods are formulated as convex optimisation problems. Three efficient spectrum sharing methods; power control, cooperative beamforming and conventional beamforming are studied in detail.

The CR power control problem is formulated as a sum rate maximisation problem and transformed into a convex optimisation problem. A robust power control method under the assumption of partial CSI is developed and also transformed into a convex optimisation problem. A novel method of detecting and removing infeasible constraints from the power allocation problem is presented that results in considerably improved performance. The performance of the proposed methods in Rayleigh fading channels is analysed by simulations.

The concept of cooperative beamforming for spectrum sharing is applied to an underlay CR relay network. Distributed single antenna relay nodes are utilised to form a virtual antenna array that provides increased gains in capacity through cooperative beamforming. It is shown that the cooperative beamforming problems can be transformed into convex optimisation problems. New robust cooperative beamformers under the assumption of partial and imperfect CSI are developed and also transformed into convex optimisation problems. The performance of the proposed methods in Rayleigh fading channels is analysed by simulations.

Conventional beamforming to allow efficient spectrum sharing in an underlay CR system is studied. The beamforming problems are formulated and transformed into convex optimisation problems. New robust beamformers under the assumption of partial and imperfect CSI are developed and also transformed into convex optimisation problems. The performance of the proposed methods in Rayleigh fading channels is analysed by simulations. 



\section{Notation and Symbols}

$\mathbb{R} \quad$ The set of real numbers

$\mathbb{R}_{+} \quad$ The set of non-negative real numbers

$\mathbb{R}_{++} \quad$ The set of positive real numbers

$\mathbb{R}^{n} \quad$ The set of real $n$-vectors

$\mathbb{R}^{m \times n} \quad$ The set of real $m \times n$ matrices

$\mathbb{C} \quad$ The set of complex numbers

$\mathbb{C}^{n} \quad$ The set of complex $n$-vectors

$\mathbb{S}^{n} \quad$ The set of symmetric $n \times n$ matrices

$\mathbb{S}_{+}^{n} \quad$ The set of symmetric positive semidefinite matrices

$\mathbb{S}_{++}^{n} \quad$ The set of symmetric positive definite matrices

dom Domain of a function

$\succeq \quad$ Element-wise greater than or equal for vectors; Positive semidefiniteness for matrices

$\succ \quad$ Element-wise greater than for vectors; Positive definiteness for matrices

$\preceq \quad$ Element-wise less than or equal for vectors; Negative semidefiniteness for matrices

$\prec \quad$ Element-wise less than for vectors; Negative definiteness for matrices

$\mathbb{E}\{\cdot \quad$ Expectation operator

$\operatorname{Pr}\{\cdot\} \quad$ Probability operator

$\mathcal{N}_{\mathcal{C}}(\cdot, \cdot) \quad$ Complex normally distributed with given mean and covariance

$\odot \quad$ Element by element Schur-Hadamard product

o Vector with all elements equal to zero

1 Vector with all elements equal to one

I Identity matrix

$\operatorname{diag}(\cdot) \quad$ Square diagnonal matrix with elements of input vector placed on the main diagonal

$(\cdot)^{H} \quad$ Complex Hermitian conjugate

$(\cdot)^{T} \quad$ Matrix or vector transpose

$|\cdot|^{2} \quad$ Magnitude squared for scalars and element-wise magnitude squared for vectors 
$\|\cdot\|_{2} \quad \ell_{2}$ norm of a vector

$(\cdot)^{1 / 2} \quad$ Square root for scalars, element-wise square root for vectors and the matrix square root for matrices

$\nabla \quad$ Vector differential operator

$\mathcal{R}(\cdot) \quad$ Range of a matrix

$\operatorname{tr}(\cdot) \quad$ Matrix trace

$\operatorname{rank}(\cdot) \quad$ Matrix rank

$\Re\{\cdot\} \quad$ Real part

$\Im\{\cdot\} \quad$ Imaginary part

$\min (\cdot) \quad$ Minimum element of a vector

$\exp (\cdot) \quad$ Exponential operator

$\log (\cdot) \quad$ Natural logarithm

$\log _{2}(\cdot) \quad$ Base 2 logarithm 


\section{Acronyms}

$\begin{array}{ll}\text { ACF } & \text { Auto-Correlation Function } \\ \text { AF } & \text { Amplify-and-Forward } \\ \text { AWGN } & \text { Additive White Gaussian Noise } \\ \text { BER } & \text { Bit Error Rate } \\ \text { CAF } & \text { Cyclic Auto-Correlation Function } \\ \text { CBD } & \text { Covariance Based Detection } \\ \text { CBF } & \text { Coordinated Downlink Beamforming } \\ \text { CDF } & \text { Cumulative Distribution Function } \\ \text { CP } & \text { Cyclic Prefix } \\ \text { CR } & \text { Cognitive Radio } \\ \text { CSD } & \text { Cyclic Spectrum Density } \\ \text { CSI } & \text { Channel State Information } \\ \text { CSIR } & \text { Channel State Information at the Receiver } \\ \text { CSIT } & \text { Channel State Information at the Transmitter } \\ \text { DF } & \text { Decode-and-Forward } \\ \text { DSA } & \text { Dynamic Spectrum Access } \\ \text { EBD } & \text { Eigenvalue Based Detection } \\ \text { ED } & \text { Energy Detector } \\ \text { FCC } & \text { Federal Communications Commission } \\ \text { GLRT } & \text { Generalised Likelihood-Ratio Test } \\ \text { GP } & \text { Geometric Program } \\ \text { HMM } & \text { Hidden Markov Model } \\ \text { KKT } & \text { Karush-Kuhn-Tucker } \\ \text { LMI } & \text { Linear Matrix Inequality } \\ \text { LP } & \text { Linear Program } \\ \text { LRT } & \text { Likelihood-Ratio Test } \\ \text { LTE } & \text { Long-Term Evolution } \\ \text { MIMO } & \text { Multiple-Input Multiple-Output } \\ \text { ML } & \text { Maximum-Likelihood } \\ \text { MSE } & \text { Mean Square Error } \\ \text { MU } & \text { Multi-User } \\ \text { MVDR } & \text { Minimum Variance Distortionless Response } \\ & \end{array}$


NF Noise Figure

OFDM Orthogonal Frequency Division Multiplexing

OSA Opportunistic Spectrum Access

OSTBC Orthogonal Space-Time Block Code

PDF Probability Density Function

PU Primary User

QCQP Quadratically Constrained Quadratic Program

QoS Quality of Service

QP Quadratic Program

SDP Semidefinite Program

SDR Semidefinite Relaxation

SICR Signal-to-Interference Channel Power Ratio

SIMO Single-Input Multiple-Output

SINR Signal-to-Interference-and-Noise Ratio

SIR Signal-to-Interference Ratio

SMI Sample Matrix Inversion

SNR Signal-to-Noise Ratio

SOCP Second-Order Cone Program

SP Stochastic Programming

SS Spectrum Sharing

SU Secondary User

TV Television

WRAN Wireless Regional Area Network 


\section{Contents}

Declaration $\quad$ i

Acknowledgements

Abstract $\quad$ v

Notation and Symbols vii

$\begin{array}{ll}\text { Acronyms } & \text { ix }\end{array}$

1 Introduction 1

1.1 Goal of this Thesis . . . . . . . . . . . . . . . 3

1.2 Structure of this Thesis . . . . . . . . . . . . . . . . . 3

2 Convex Optimisation $\quad 7$

2.1 Convex Sets . . . . . . . . . . . . . . . . . . . 7

2.1 .1 Cones . . . . . . . . . . . . . . . . . . 8

2.1.2 Hyperplanes and Halfspaces . . . . . . . . . . . . . . 8

2.1.3 Euclidean Balls and Ellipsoids . . . . . . . . . . . . 8

2.1.4 Norm balls and Norm Cones . . . . . . . . . . . . . . . . . . 9

2.1.5 Polyhedra . . . . . . . . . . . . . . . . 9 9

2.1.6 Positive Semidefinite Cone . . . . . . . . . . . . . . . . 9

2.2 Convex Functions . . . . . . . . . . . . . . . . . . . . . . . . . . 10

2.2.1 Examples of Convex Functions . . . . . . . . . . . . . 10

2.3 Convex Optimisation Problems . . . . . . . . . . . . . . . 11

2.4 Classes of Convex Optimisation Problems . . . . . . . . . . . . . 12

2.4 .1 Linear Program . . . . . . . . . . . . . . . . . . 12

2.4.2 Linear-fractional Program . . . . . . . . . . . . . . . . 12

2.4.3 Quadratic Program . . . . . . . . . . . . . 13

2.4.4 Quadratically Constrained Quadratic Program . . . . . . . 13

2.4.5 Second-order Cone Program . . . . . . . . . . . . . . . . . . 14

2.4.6 Geometric Program . . . . . . . . . . . . . . . . . . . 14

2.4.7 Semidefinite Program . . . . . . . . . . . . . 15

2.5 Optimality, Duality and Karush-Kuhn-Tucker (KKT) Conditions . . 15 
2.5.1 Optimality Criterion . . . . . . . . . . . . 15

2.5 .2 Duality ...................... 16

2.5.3 Karush-Kuhn-Tucker (KKT) Conditions . . . . . . . . . . 17

2.6 The Epigraph Form . . . . . . . . . . . . . . . . . . . . . . . . . . 18

2.7 Convex Relaxations of Non-convex Problems . . . . . . . . . . . . . 19

2.7.1 Semidefinite Relaxation (SDR) . . . . . . . . . 20

2.7 .2 Lagrangian Relaxation . . . . . . . . . . . . . . . . . . 21

2.7.3 Extracting a Rank-1 Solution . . . . . . . . . . . . . 22

2.8 Algorithms For Solving Convex Optimisation Problems . . . . . . . 22

2.8.1 A Brief History . . . . . . . . . . . . . . . . 23

2.8.2 Unconstrained Minimisation . . . . . . . . . . . . . 24

2.8.3 Descent Algorithms . . . . . . . . . . . . . . . . . . 25

2.8.4 Self-concordance . . . . . . . . . . . . . 27

2.8.5 Equality Constrained Minimisation . . . . . . . . . . . 27

2.8.6 Interior-point Methods . . . . . . . . . . . . . . . . . 28

2.9 Summary . . . . . . . . . . . . . . . . . . . . . . . 32

3 Robust Optimisation $\quad 33$

3.1 Bounded Uncertainty Based Robust Optimisation . . . . . . . . . . 34

3.1 .1 Ellipsoidal Uncertainty . . . . . . . . . . . . . . . . . 34

3.1 .2 The S-Procedure . . . . . . . . . . . . . . 36

3.2 Robust Stochastic Optimisation . . . . . . . . . . . . . . . . 37

3.3 Application of Robust Optimisation in Communication Systems . . 40

3.3.1 Power Control in Wireless Networks . . . . . . . . . . . . . . 40

3.3.2 Receive Beamforming . . . . . . . . . . . . . . 44

3.3 .3 Transmit Beamforming . . . . . . . . . . . . . 50

3.3.4 Relay Beamforming . . . . . . . . . . . . . . . 55

3.4 Summary . . . . . . . . . . . . . . . . . . 60

4 Cognitive Radio Systems $\quad 61$

4.1 Spectrum Sensing for OSA . . . . . . . . . . . . . . . . . 62

4.1 .1 Energy Detection . . . . . . . . . . . . . . 64

4.1.2 Matched Filter Detection . . . . . . . . . . . . . 65

4.1.3 Sensing Based on Feature Detection . . . . . . . . . . . . 65

4.1 .4 Blind Detection . . . . . . . . . . . . . . . 67

4.2 Spectrum Sharing . . . . . . . . . . . . . . . . 68

4.2.1 Transmit Power Control . . . . . . . . . . . . . . . 69

4.2.2 Transmit Beamforming . . . . . . . . . . . . . . . . 69

4.3 Summary . . . . . . . . . . . . . . . . . . 70 
5 Power Control in Underlay Cognitive Radio Systems 71

5.1 Introduction . . . . . . . . . . . . . . . . . . . . . . . . 71

5.2 System Model . . . . . . . . . . . . . . . . . . 73

5.3 SU Power Optimisation . . . . . . . . . . . . . . . . . . 75

5.3.1 High SINR Scenario . . . . . . . . . . . . 76

5.3 .2 Low SINR Scenario . . . . . . . . . . . . . . 77

5.4 SU Power Optimisation with Feasibility Detection . . . . . . . . . . 79

5.5 SU Power Optimisation Under Channel Uncertainties . . . . . . . . 80

5.6 SU Power Optimisation With Feasibility Detection Under Channel Uncertainties . . . . . . . . . . . . . . . . . . 81

5.7 Simulation Results and Discussion . . . . . . . . . . . . . . 82

5.8 Summary . . . . . . . . . . . . . . . . . . . . . . . . . . . . . . . 91

6 Robust Cognitive Radio Cooperative Beamforming 95

6.1 Introduction . . . . . . . . . . . . . . . . . . . . 95

6.2 System Model . . . . . . . . . . . . . . . . . . . . . 97

6.3 Beamformer Optimisation . . . . . . . . . . . . . . 100

6.3.1 Relay Power Minimisation . . . . . . . . . . . . . . . 101

6.3.2 Secondary Receiver SINR Maximisation . . . . . . . . . . . 102

6.4 Robust Beamformer Optimisation . . . . . . . . . . . . . . 105

6.4.1 Partial CSI Availability for the $\mathrm{PU}_{\mathrm{Tx}}$ to $\mathrm{PU}_{\mathrm{Rx}}$ Link . . . . . 106

6.4.2 Partial CSI Availability for the $\mathrm{PU}_{\mathrm{Tx}}$ to $\mathrm{PU}_{\mathrm{Rx}}$ and $\mathrm{SU}_{\mathrm{Rl}}$ to $\mathrm{PU}_{\mathrm{Rx}}$ Links . . . . . . . . . . . . . . . 107

6.4.3 Partial CSI Availability for the $\mathrm{PU}_{\mathrm{Tx}}$ to $\mathrm{PU}_{\mathrm{Rx}}$ Link and Imperfect CSI Availability for the $\mathrm{SU}_{\mathrm{Rl}}$ to $\mathrm{PU}_{\mathrm{Rx}}$ Links . . . . . 113

6.5 Simulation Results and Discussion . . . . . . . . . . . . . . . . 120

6.6 Summary . . . . . . . . . . . . . . . . . . . 127

7 Robust Cognitive Radio Beamforming $\quad 129$

7.1 Introduction . . . . . . . . . . . . . . . . . . . . . . 129

7.2 System Model . . . . . . . . . . . . . . . . . . . . . . . . 131

7.3 Beamformer Optimisation Under Full CSI . . . . . . . . . . . . . 132

7.3.1 SU Transmitter Power Minimisation . . . . . . . . . . . . 133

7.3.2 SU Receiver SNR Maximisation . . . . . . . . . . . . . . . . 134

7.3.3 Minimum PU Receiver SINR Maximisation . . . . . . . . . . 134

7.4 Robust Beamformer Optimisation Under Partial And Imperfect CSI 135

7.4.1 Robust SU Transmitter Power Minimisation . . . . . . . . . 138

7.4.2 Robust SU Outage Probability Minimisation . . . . . . . . . 141

7.4.3 Robust Maximum PU Outage Probability Minimisation . . . 142

7.5 Simulation Results and Discussion . . . . . . . . . . . . . . . 144

7.6 Summary . . . . . . . . . . . . . . . . . . . 152 
8 Conclusions and Further Research

155

8.1 Conclusions . . . . . . . . . . . . . . . . . . . . 155

8.2 Discussion . . . . . . . . . . . . . . . . . . . . . 157

8.3 Suggestions for Future Research . . . . . . . . . . . . . . . . . 159 


\section{Chapter 1}

\section{Introduction}

The last decade has seen a phenomenal increase in consumer usage of smartphones, tablets, laptops and other mobile devices. This has led to an exponential increase of mobile data traffic as consumers surf the web, check email, use apps and watch and send videos and photos through these mobile devices. According to Cisco's global mobile data traffic forecast [47], the industry's most comprehensive annual study, in 2013,

- Global mobile data traffic grew more than $80 \%$ from 820 petabytes per month to 1.5 exabytes per month.

- Mobile data traffic was nearly 18 times the size of the entire global Internet traffic in 2000.

The above statistics demonstrates the consumers' insatiable appetite for mobile data, but the story does not end there. In [47], it is forecasted that by 2018,

- Global mobile data traffic will exceed 15 exabytes per month.

- Smartphones will reach $66 \%$ of mobile data traffic.

- Mobile tablet traffic will surpass 2.5 exabytes per month.

- $4 \mathrm{G}$ traffic will be more than half of the total mobile traffic.

After analysing the projected mobile traffic data, a question that naturally arises is; aren't there limits to how much data the mobile networks can support? The upper limit is of course given by Shannon's channel capacity theorem [207], which states that the capacity of a channel is directly proportional to the bandwidth of the channel. A 2010 report by the Federal Communications Commission (FCC) [49] outlined that the growth in wireless data demand will lead to a spectrum deficit, colloquially known as the spectrum crunch, where user demand will outstrip the capacity of the radio spectrum. Although new spectrally efficient technologies, such as LTE [57] provide some relief, this alone will not be enough to meet user 
demand. The FCC has therefore advocated for the release of more spectrum [49] and the more efficient use of currently allocated spectrum [48].

In 2002, a report aimed at improving the manner in which spectrum is managed in the United States was published by the FCC Spectrum Policy Task Force [48]. One of the main findings of this report was that in many bands, spectrum access rather than the physical scarcity of spectrum is a significant problem and there are substantial unused resources in frequency, time and space. The efficiency of spectrum utilisation can be significantly improved if unlicensed users are allowed to access the spectrum when it is unoccupied by the incumbent users. Cognitive radio (CR) $[102,163,164]$ has been proposed as an intelligent and effective technology for exploiting underutilised spectral resources by reusing unused spectrum in a dynamic and opportunistic manner. The use of CRs will inevitably create increased interference and thus degrade the performance of the licensed users. To maintain the impact to an acceptable level, this interference must be intelligently managed by the CR systems. This can be achieved in two ways. Firstly, the spectrum could be used in a mutually exclusive manner. Here, the CRs sense the spectrum to check for availability and only use it when it is deemed to be free. Secondly, the CRs could use the spectrum concurrently (spectrum sharing) with the licensed users, provided the interference is kept within some acceptable limits. This could be achieved by controlling the transmission parameters, such as the transmit power or the transmit direction, of the CR system. Through an appropriately formulated optimisation problem, the transmission parameters could be designed to achieve the goals of the CR system while providing guaranteed quality of service (QoS) to the licensed users.

Convex optimisation [29] is one of several optimisation techniques that could be used to solve CR spectrum sharing problems. The main advantage of a convex formulation of a problem is that the optimisation problem has only one minimum, which is the global minimum. Hence, convex optimisation problems can always be solved, either analytically or numerically, to obtain the optimum solution. For practical systems, any solutions obtained need to be robust against uncertainty in input data. Solutions to many optimisation problems suffer from sensitivity to uncertain data and even minor uncertainties can render the problems suboptimal or even infeasible [21]. In wireless communications, it is common to have imperfect or partial channel state information (CSI). The imperfections arise due to estimation errors or other factors such as quantisation. Therefore, any spectrum sharing optimisation problems developed for CR systems need to take into consideration the uncertainty in CSI in order to guarantee QoS to the licensed users. 


\subsection{Goal of this Thesis}

The goal of this thesis is to develop effective methods for spectrum sharing in cognitive radio systems by formulating and solving the following problems as convex optimisation problems:

1. robust power allocation in networks with single antenna transmitters and receivers,

2. robust cooperative beamforming in relay networks where single antenna nodes cooperate to form a virtual antenna array, and

3. robust conventional beamforming in networks where the transmitter is equipped with multiple antennas.

The main focus is on developing techniques that are robust against uncertainty in the CSI.

In this thesis, these spectrum sharing methods are presented in the above order for two reasons. Firstly, this organisation provides a logical progression from single antenna systems to multiple antenna systems. Secondly, the insights gained through solving the robust cooperative beamforming problem are applied to the solution of the conventional robust beamforming problem.

\subsection{Structure of this Thesis}

Chapters 2-4 present important background material, and Chapters 5-7 present original research.

A review of convex optimisation theory and techniques is presented in Chapter 2. Many communication problems can be either cast as or be converted into convex optimisation problems. In order to recognise or convert communication problems as convex optimisation problems, one must be familiar with the concepts of convexity and the "tricks of the trade" that allow seemingly non-convex problems to be easily transformed into convex problems. The concepts of convex sets, convex functions and convex optimisation problems are introduced. A review of Lagrange duality and the well known Karush-Kuhn-Tucker conditions for optimality are provided. A powerful technique known as convex relaxation, which allows non-convex problems to be relaxed into convex problems is reviewed. The chapter concludes with a review of algorithms for solving convex optimisation problems.

An overview of robust optimisation techniques is provided in Chapter 3. Robust optimisation is generally used when there is some degree of uncertainty in the input data. The uncertainty may arise due to noise, measurement errors or partial knowledge of the data. A review of the two most widely used robust optimisation 
methods, the bounded uncertainty based optimisation and stochastic optimisation, is provided. Application of these methods to communication problems, specifically, power control, conventional receive and transmit beamforming and cooperative relay beamforming, is presented. This chapter lays the foundation needed for the development of the problems posed in Chapters 5-7.

Chapter 4 discusses the cognitive radio concept and dynamic spectrum access techniques. The state of the art spectrum sensing algorithms and spectrum sharing methods are reviewed.

In Chapter 5, power allocation problems for multiple cognitive radio users sharing spectrum with a pair of primary (licensed) users are formulated. The problems are transformed into convex optimisation problems and solved numerically using standard convex optimisation solution methods. A novel method of detecting and removing cognitive users who are unable to satisfy their QoS requirements is proposed. Using a stochastic optimisation approach, robust power allocation problems under the assumption of partial CSI are developed. The robust problems are shown to be convex optimisation problems and solved numerically using standard convex optimisation solution methods. Results in the form of sum rate cumulative distribution functions (CDF) for various Rayleigh fading channels are presented.

Chapter 6 considers the problem of cooperative beamforming in a cognitive radio relay network that shares spectrum with a pair of primary users. Due to poor channel conditions, the cognitive source is unable to communicate directly with the cognitive destination and hence employs the cognitive relays for assistance. All transmitters and receivers are assumed to be equipped with only single antennas. The idea of cooperative beamforming is applied to the geographically distributed cognitive relay nodes which cooperate to beamform towards the cognitive destination while maintaining the interference generated at the primary user below some acceptable level. Firstly, under the assumption of the availability of perfect CSI for all links, the beamforming problems are formulated as convex optimisation problems and solved numerically using standard convex optimisation solution methods. Secondly, under the assumption of partial and imperfect CSI at the cognitive system, a stochastic optimisation approach is taken and new robust cognitive cooperative relay beamformers are proposed. These robust problems are transformed into convex optimisation problems and solved numerically using standard convex optimisation solution methods. CDFs of primary receiver and cognitive destination receiver signal-to-interference-and-noise ratio (SINR) for Rayleigh fading channels are presented.

In Chapter 7, robust cognitive radio beamformers are developed for a cognitive transmitter equipped with multiple antennas that shares spectrum with multiple primary user pairs. A stochastic approach is taken and new robust beamformers are developed under the assumption of partial and imperfect CSI. The beamforming 
problems are shown to be convex optimisation problems and solved numerically using standard convex optimisation solution methods. The performance of the proposed robust beamformers are demonstrated through CDFs of primary receiver SINR and cognitive receiver signal-to-noise ratio (SNR) in Rayleigh fading channels.

The thesis is concluded by Chapter 8, which summarises the original contributions of the preceding chapters and suggests topics for future research. 



\section{Chapter 2}

\section{Convex Optimisation}

Convex optimisation has been studied by mathematicians for over a century [29]. Recently it has been recognised as a powerful tool by the signal processing community [146]. Convex optimisation involves minimisation of a convex objective function subject to convex constraints. There are numerous applications, some of which include estimation and signal processing [17], communications and networks [25, 149, 216], electronic circuit design [74, 195], automatic control systems [30], statistics [29], and finance [192].

There are many advantages of formulating a problem as a convex optimisation problem. Some of these include:

- Any local minima of convex problems is also the global minimum.

- Convex problems can always be solved numerically even though a closed form solution may not exist.

- Constraints can be easily added to the problem.

- Convex problems can be solved very reliably and efficiently using interiorpoint methods in polynomial-time $[62,175]$.

Many problems that arise in communications signal processing can be cast or converted into convex optimisation problems which allow analytical or numerical solutions to be calculated easily. This chapter provides an overview of convex optimisation theory and techniques.

\subsection{Convex Sets}

A set $C$ is convex if a line segment between any two points $C$ lies in $C$ [29, p. 21]. This can be mathematically represented as

$$
\theta x_{1}+(1-\theta) x_{2} \in C,
$$



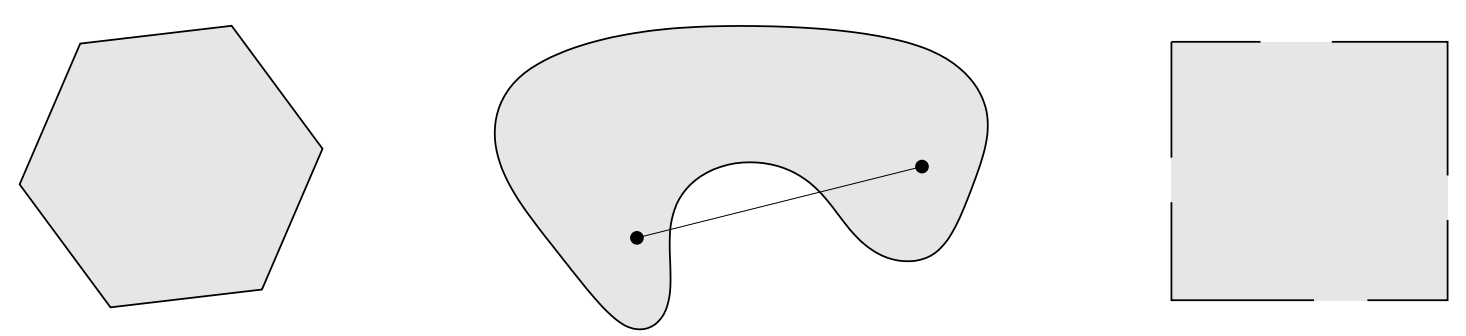

Figure 2.1: Convex and non-convex sets. Left. The hexagon which includes its boundary is convex. Middle. The kidney shaped set is not convex. Right. The square contains some boundary points but not others hence is not convex. Reproduced from [29].

for any $x_{1}, x_{2} \in C$ and $0 \leq \theta \leq 1$. Examples of convex and non-convex sets are shown in Fig. 2.1. Lines and line segments also form convex sets.

\subsubsection{Cones}

A set $C$ is called a cone if for every $x \in C$ and $\theta \geq 0, \theta x \in C$ [29, p. 25]. If the set $C$ is convex and a cone then it is called a convex cone. Convex cones satisfy the following condition

$$
\theta_{1} x_{1}+\theta_{2} x_{2} \in C
$$

for any $x_{1}, x_{2} \in C$ and $\theta_{1}, \theta_{2} \geq 0$. The non-negative orthant is an example of a convex cone.

\subsubsection{Hyperplanes and Halfspaces}

Hyperplanes and halfspaces form convex sets. A hyperplane is given by [29, p. 27]

$$
\left\{\mathbf{x} \mid \mathbf{a}^{T} \mathbf{x}=b\right\}
$$

and a halfspace by

$$
\left\{\mathbf{x} \mid \mathbf{a}^{T} \mathbf{x} \leq b\right\}
$$

where $\mathbf{a} \in \mathbb{R}^{n}, \mathbf{a} \neq 0$ and $b \in \mathbb{R}$.

\subsubsection{Euclidean Balls and Ellipsoids}

Euclidean balls and ellipsoids form convex sets. A Euclidean ball in $\mathbb{R}^{n}$ is given by $[29$, p. 29]

$$
B\left(\mathbf{x}_{c}, r\right)=\left\{\mathbf{x} \mid\left\|\mathbf{x}-\mathbf{x}_{c}\right\|_{2} \leq r\right\}
$$

where $\mathbf{x}_{c}$ is the center of the ball and $r>0$ is its radius. Hence, $B\left(\mathbf{x}_{c}, r\right)$ consists of all points within a distance $r$ of the center $\mathbf{x}_{c}$. 
Ellipsoids have the form

$$
\mathcal{E}=\left\{\mathbf{x} \mid\left(\mathbf{x}-\mathbf{x}_{c}\right)^{T} \mathbf{P}^{-1}\left(\mathbf{x}-\mathbf{x}_{c}\right) \leq 1\right\}
$$

where $\mathbf{P}$ is a symmetric and positive definite matrix and $\mathbf{x}_{c}$ the center of the ellipsoid. By taking $\mathbf{A}=\mathbf{P}^{\frac{1}{2}}$, ellipsoids can also be represented as

$$
\mathcal{E}=\left\{\mathbf{x}_{c}+\mathbf{A} \mathbf{u} \mid\|\mathbf{u}\|_{2} \leq 1\right\}
$$

\subsubsection{Norm balls and Norm Cones}

Norm balls and norm cones form convex sets. A norm ball of radius $r$ and center at $\mathbf{x}_{c}$ is given by $[29$, p. 30$]$

$$
B\left(\mathbf{x}_{c}, r\right)=\left\{\mathbf{x} \mid\left\|\mathbf{x}-\mathbf{x}_{c}\right\| \leq r\right\}
$$

where $\|\cdot\|$ is any norm on $\mathbb{R}^{n}$. The norm cone associated with the norm $\|\cdot\|$ is the set

$$
C=\{(\mathbf{x}, t) \mid\|\mathbf{x}\| \leq t\} \subseteq \mathbb{R}^{n+1},
$$

where $t>0$. The second-order cone is the norm cone for the Euclidean norm. It is also known as the Lorentz cone or ice-cream cone.

\subsubsection{Polyhedra}

A polyhedron is the intersection of a finite number of hyperplanes and halfspaces [29, p. 31]. It forms a convex set.

\subsubsection{Positive Semidefinite Cone}

Positive semidefinite matrices form a convex cone, hence a convex set [29, p. 34]. This can be proven using (2.2) and the definition of positive semidefiniteness as follows. For any $\theta_{1}, \theta_{2} \geq 0, \mathbf{A}, \mathbf{B} \in \mathbb{S}_{+}^{n}$ and $\mathbf{x} \in \mathbb{R}^{n}$, we have

$$
\mathbf{x}^{T}\left(\theta_{1} \mathbf{A}+\theta_{2} \mathbf{B}\right) \mathbf{x}=\theta_{1} \mathbf{x}^{T} \mathbf{A} \mathbf{x}+\theta_{2} \mathbf{x}^{T} \mathbf{A} \mathbf{x} \geq 0
$$

Hence $\theta_{1} \mathbf{A}+\theta_{2} \mathbf{B} \in \mathbb{S}_{+}^{n}$, which proves that the set of positive semidefinite matrices form a convex cone. 


\subsection{Convex Functions}

A function $f: \mathbb{R}^{n} \rightarrow \mathbb{R}$ is convex if its domain is a convex set and for any two points $\mathbf{x}, \mathbf{y} \in \operatorname{dom} f$, and $\theta$ with $0 \leq \theta \leq 1$, we have [29, p. 67]

$$
f(\theta \mathbf{x}+(1-\theta) \mathbf{y}) \leq \theta f(\mathbf{x})+(1-\theta) f(\mathbf{y})
$$

This inequality implies that the line segment between $(\mathbf{x}, f(\mathbf{x}))$ and $(\mathbf{y}, f(\mathbf{y}))$ always lies above the function $f$. A function $f$ is concave if $-f$ is convex.

The first-order and second-order conditions can also be used to test the convexity of a function. The first-order condition states that a differentiable function $f$ is convex if and only if $\operatorname{dom} f$ is convex and

$$
f(\mathbf{y}) \geq f(\mathbf{x})+\nabla f(\mathbf{x})^{T}(\mathbf{y}-\mathbf{x})
$$

The right hand side of the inequality (2.12) is the first-order Taylor approximation of $f$ near $\mathbf{x}$. This inequality states that for a convex function, the first-order Taylor approximation is a global under-estimator of the function.

The second-order condition states that if a function $f$ is twice differentiable, i.e., its Hessian or second derivative $\nabla^{2} f$ exists at each point in $\operatorname{dom} f$ then, $f$ is convex if and only if $\operatorname{dom} f$ is convex and its Hessian is positive semidefinite [29, p. 71], i.e.,

$$
\nabla^{2} f(\mathbf{x}) \succeq 0, \quad \forall \mathbf{x} \in \operatorname{dom} f
$$

\subsubsection{Examples of Convex Functions}

Listed below are examples of convex functions [29, p. 71$]$

- $\exp (a x)$ is convex on $\mathbb{R}$, for any $a \in \mathbb{R}$.

- $x^{a}$ is convex on $\mathbb{R}_{++}$when $a \geq 1$ or $a \leq 0$, and concave for $0 \leq a \leq 1$.

- $|x|^{p}$ is convex on $\mathbb{R}$ for $p \geq 1$.

- $\log (x)$ is concave on $\mathbb{R}_{++}$.

- $x \log (x)$ is convex on $\mathbb{R}_{++}$or on $\mathbb{R}_{+}$.

- Every norm on $\mathbb{R}^{n}$ is convex.

- $\max \left\{x_{1}, \ldots, x_{n}\right\}$ is convex on $\mathbb{R}^{n}$.

- A quadratic function $f(\mathbf{x})=\mathbf{x}^{T} \mathbf{P} \mathbf{x}+\mathbf{a}^{T} \mathbf{x}+b$ is convex if $\mathbf{P} \succeq 0$ (second-order condition). 
- The geometric mean $f(\mathbf{x})=\left(\prod_{i=1}^{n} x_{i}\right)^{\frac{1}{n}}$, where $x_{i}$ is the $i$ th element of $\mathbf{x}$, is concave on $\mathbb{R}_{++}^{n}$.

\subsection{Convex Optimisation Problems}

A generic optimisation problem (in minimisation form) is specified as [29, p. 127]

$$
\begin{array}{cl}
\min _{\mathbf{x}} & f_{0}(\mathbf{x}) \\
\text { s.t. } & f_{i}(\mathbf{x}) \leq 0, \quad i=1 \ldots m \\
& h_{i}(\mathbf{x})=0, \quad i=1 \ldots p
\end{array}
$$

The aim of the optimisation problem is to find the $\mathbf{x}$ that minimises $f_{0}(\mathbf{x})$ while satisfying $f_{i}(\mathbf{x}) \leq 0, i=1 \ldots m$, and $h_{i}(\mathbf{x})=0, \quad i=1 \ldots p$. The variable $\mathbf{x} \in \mathbb{R}^{n}$ is called the optimisation variable and the function $f_{0}: \mathbb{R}^{n} \rightarrow \mathbb{R}$ is known as the objective function. The inequalities $f_{i}(\mathbf{x}) \leq 0$ are referred to as inequality constraints and the functions $f_{i}: \mathbb{R}^{n} \rightarrow \mathbb{R}$ are called inequality constraint functions. The equalities $h_{i}(\mathbf{x})=0$ are called equality constraints and the functions $h_{i}: \mathbb{R}^{n} \rightarrow$ $\mathbb{R}$ are known as equality constraint functions.

The domain $\mathcal{D}$ of the optimisation problem is the set of points for which the objective and constraint functions are defined. A point $\mathbf{x} \in \mathcal{D}$ is called a feasible point if all constraints are satisfied. The optimisation problem is feasible if there is at least one feasible point.

A feasible solution $\mathbf{x}^{*}$ is called a globally optimum solution if $f_{0}\left(\mathbf{x}^{*}\right) \leq f_{0}(\mathbf{x})$ for all feasible $\mathbf{x}$. A feasible solution $\overline{\mathbf{x}}$ is called a locally optimum solution if there exists an $\epsilon>0$ such that $f_{0}(\overline{\mathbf{x}}) \leq f_{0}(\mathbf{x})$ for all feasible $\mathbf{x}$ that satisfies $\|\mathbf{x}-\overline{\mathbf{x}}\|_{2} \leq \epsilon$. A fundamental property of convex optimisation problems is that any locally optimal point is also globally optimal.

An optimisation problem is convex if and only if all of the following conditions are satisfied.

- The objective function is convex.

- The inequality constraint functions are convex.

- The equality constraint functions are affine, i.e., have the form $h_{i}(\mathbf{x})=a_{i}^{T} \mathbf{x}+$ $b_{i}$.

- The domain of the optimisation problem is convex. 


\subsection{Classes of Convex Optimisation Problems}

In this section a number of common classes of convex optimisation problems are introduced.

\subsubsection{Linear Program}

An optimisation problem with affine objective and constraint functions is known as a linear program (LP) $[29$, p. 146]. An LP in the general form is specified as

$$
\begin{array}{cl}
\min _{\mathbf{x}} & \mathbf{c}^{T} \mathbf{x}+d \\
\text { s.t. } & \mathbf{G} \mathbf{x} \preceq \mathbf{h}, \\
& \mathbf{A x}=\mathbf{b},
\end{array}
$$

where $\mathbf{x} \in \mathbb{R}^{n}, \mathbf{c} \in \mathbb{R}^{n}, d \in \mathbb{R}, \mathbf{G} \in \mathbb{R}^{m \times n}, \mathbf{h} \in \mathbb{R}^{m}, \mathbf{A} \in \mathbb{R}^{p \times n}$ and $\mathbf{b} \in \mathbb{R}^{p}$.

Geometrically, an LP represents the minimisation of an affine function over a polyhedron feasible set.

\subsubsection{Linear-fractional Program}

A linear-fractional program is specified as [29, p. 151]

$$
\begin{array}{cl}
\min _{\mathbf{x}} & \frac{\mathbf{c}^{T} \mathbf{x}+d}{\mathbf{e}^{T} \mathbf{x}+f} \\
\text { s.t. } & \mathbf{G x} \preceq \mathbf{h}, \\
& \mathbf{A x}=\mathbf{b},
\end{array}
$$

where $\mathbf{x} \in \mathbb{R}^{n}, \mathbf{c}, \mathbf{e} \in \mathbb{R}^{n}, d, f \in \mathbb{R}, \mathbf{G} \in \mathbb{R}^{m \times n}, \mathbf{h} \in \mathbb{R}^{m}, \mathbf{A} \in \mathbb{R}^{p \times n}, \mathbf{b} \in \mathbb{R}^{p}$ and the domain of the objective function is $\left\{\mathbf{x} \mid \mathbf{e}^{T} \mathbf{x}+f>0\right\}$.

The objective function of problem (2.16) is a non-convex function, hence the problem is a non-convex optimisation problem. However, the Charnes-Cooper transformation [42] can be used to transform problem (2.16) into an equivalent LP. The Charnes-Cooper transformation first defines the pair

$$
\mathbf{y}=\frac{\mathbf{x}}{\mathbf{e}^{T} \mathbf{x}+f}, \quad z=\frac{1}{\mathbf{e}^{T} \mathbf{x}+f}
$$


which is then substituted into problem (2.16) to obtain the LP

$$
\begin{array}{cl}
\min _{\mathbf{y}, z} & \mathbf{c}^{T} \mathbf{y}+d z \\
\text { s.t. } & \mathbf{G y}-\mathbf{h} z \preceq \mathbf{0}, \\
& \mathbf{A y}-\mathbf{b} z=\mathbf{0}, \\
& \mathbf{e}^{T} \mathbf{y}+f z=1, \\
& z \geq 0 .
\end{array}
$$

\subsubsection{Quadratic Program}

A convex optimisation problem is called a quadratic program (QP) if the objective is a convex quadratic function and the constraint functions are all affine [29, p. 152]. A QP is expressed as

$$
\begin{array}{cl}
\min _{\mathbf{x}} & \mathbf{x}^{T} \mathbf{P} \mathbf{x}+\mathbf{q}^{T} \mathbf{x}+r \\
\text { s.t. } & \mathbf{G} \mathbf{x} \preceq \mathbf{h}, \\
& \mathbf{A x}=\mathbf{b},
\end{array}
$$

where $\mathbf{x} \in \mathbb{R}^{n}, \mathbf{P} \in \mathbb{S}_{+}^{n}, \mathbf{q} \in \mathbb{R}^{n}, r \in \mathbb{R}, \mathbf{G} \in \mathbb{R}^{m \times n}, \mathbf{h} \in \mathbb{R}^{m}, \mathbf{A} \in \mathbb{R}^{p \times n}$ and $\mathbf{b} \in \mathbb{R}^{p}$.

Geometrically, a QP represents the minimisation of a convex quadratic function over a polyhedron feasible set. The least squares optimisation problem is possibly the most well known QP.

\subsubsection{Quadratically Constrained Quadratic Program}

An optimisation problem is known as a quadratically constrained quadratic program (QCQP) when the objective and inequality constraint functions are all convex quadratics [29, p. 152]. A QCQP is expressed as

$$
\begin{array}{ll}
\min _{\mathbf{x}} & \mathbf{x}^{T} \mathbf{P}_{0} \mathbf{x}+\mathbf{q}_{0}^{T} \mathbf{x}+r_{0} \\
\text { s.t. } & \frac{1}{2} \mathbf{x}^{T} \mathbf{P}_{i} \mathbf{x}+\mathbf{q}_{i}^{T} \mathbf{x}+r_{i} \leq 0, \quad i=1 \ldots m \\
& \mathbf{A} \mathbf{x}=\mathbf{b},
\end{array}
$$

where $\mathbf{x} \in \mathbb{R}^{n}, \mathbf{P}_{i} \in \mathbb{S}_{+}^{n}, i=0,1 \ldots, m, \mathbf{q}_{i} \in \mathbb{R}^{n}, r_{i} \in \mathbb{R}, \mathbf{A} \in \mathbb{R}^{p \times n}$ and $\mathbf{b} \in \mathbb{R}^{p}$. 


\subsubsection{Second-order Cone Program}

The second-order cone program (SOCP) is expressed as [29, p. 156]

$$
\begin{array}{cl}
\min _{\mathbf{x}} & f^{T} \mathbf{x} \\
\text { s.t. } & \left\|\mathbf{A}_{i} \mathbf{x}+\mathbf{b}_{i}\right\|_{2} \leq \mathbf{c}_{i}^{T} \mathbf{x}+d_{i}, \quad i=1 \ldots m \\
& \mathbf{F} \mathbf{x}=\mathbf{g},
\end{array}
$$

where $\mathbf{x} \in \mathbb{R}^{n}, \mathbf{f} \in \mathbb{R}^{n}, \mathbf{A}_{i} \in \mathbf{R}^{n_{i} \times n}, \mathbf{b}_{i} \in \mathbf{R}^{n_{i}}, \mathbf{c}_{i} \in \mathbb{R}^{n}, d_{i} \in \mathbb{R}, \mathbf{F} \in \mathbb{R}^{p \times n}$ and $\mathbf{g} \in \mathbb{R}^{p}$.

The inequality constraint, $\left\|\mathbf{A}_{i} \mathbf{x}+\mathbf{b}_{i}\right\|_{2} \leq \mathbf{c}_{i}^{T} \mathbf{x}+d_{i}$ is called a second-order cone constraint since it represents a second-order (norm) cone (Section 2.1.4).

The SOCP is equivalent to a QCQP when $c_{i}=0, \forall i$ and it reduces to a LP when $\mathbf{A}_{i}=0, \forall i$.

\subsubsection{Geometric Program}

Geometric programs (GP) are a class of optimisation problems that are not convex; however, they can be transformed to convex optimisation problems through a change of variables and a transformation of the objective and constraint functions $[8,18,29]$. We first introduce two important functions, monomials and posynomials, before delving further into GPs.

A monomial is a function $f: \mathbf{R}_{++}^{n} \rightarrow \mathbf{R}$, with $\operatorname{dom} f=\mathbb{R}_{++}^{n}$, defined as [29, p. 160]

$$
f(\mathbf{x})=c x_{1}^{a_{1}} x_{2}^{a_{2}} \cdots x_{n}^{a_{n}}
$$

where $x_{i}$ is the $i$ th element of the vector $\mathbf{x}, c>0$ and $a_{i} \in \mathbb{R}, i=1,2, \ldots, n$ are exponents. A sum of monomial functions is called a posynonomial. A posynomial takes the form [29, p. 160]

$$
f(\mathbf{x})=\sum_{k=1}^{K} c_{k} x_{1}^{a_{1 k}} x_{2}^{a_{2 k}} \cdots x_{n}^{a_{n k}},
$$

where $c_{k}>0$, and $a_{i k} \in \mathbf{R}, i=1,2, \ldots, n$.

A GP is stated as the following optimisation problem:

$$
\begin{array}{ll}
\min _{\mathbf{x}} & f_{0}(\mathbf{x}) \\
\text { s.t. } & f_{i}(\mathbf{x}) \leq 1, \quad i=1, \ldots, m \\
& h_{i}(\mathbf{x})=1, \quad i=1, \ldots, p
\end{array}
$$

where $f_{0}, \ldots, f_{m}$ are posynomials: $f_{i}(\mathbf{x})=\sum_{k=1}^{K_{i}} c_{i k} x_{1}^{a_{i k}^{(1)}} x_{2}^{a_{i k}^{(2)}} \ldots x_{n}^{a_{i k}^{(n)}}$ and $h_{1}, \ldots, h_{p}$ 
are monomials: $h_{i}(\mathbf{x})=c_{i} x_{1}^{a_{i}^{(1)}} x_{2}^{a_{i}^{(2)}} \ldots x_{n}^{a_{i}^{(n)}}$. The GP in (2.23) is a non-convex optimisation problem but can be transformed into a convex problem by a logarithmic change of variables and by taking the logarithm of the objective and constraint functions. This results in the problem [29, p. 162]

$$
\begin{array}{lll}
\min _{\mathbf{y}} & \tilde{f}_{0}(\mathbf{y})=\log \left(\sum_{k=1}^{K_{0}} e^{\mathbf{a}_{0 k}^{T} \mathbf{y}+b_{0 k}}\right), & \\
\text { s.t. } & \tilde{f}_{i}(\mathbf{y})=\log \left(\sum_{k=1}^{K_{i}} e^{\mathbf{a}_{i k}^{T} \mathbf{y}+b_{i k}}\right) \leq 0, & i=1, \ldots, m \\
& \tilde{h}_{i}(\mathbf{y})=\mathbf{g}_{i}^{T} \mathbf{y}+d_{i}=0, & i=1, \ldots, p
\end{array}
$$

where $y_{i}=\log x_{i}, i=0, \ldots, n, b_{i k}=\log c_{i k}, i=0, \ldots, m$, and $d_{i}=\log c_{i}, i=$ $1, \ldots, p$. The vectors $\mathbf{a}_{i k} \in \mathbf{R}^{n}, i=0, \ldots, m$, contain the exponents of the posynomials and the vectors $\mathbf{g}_{\mathbf{i}} \in \mathbf{R}^{n}, i=1, \ldots, p$, contain the exponents of the monomials. Since the functions $\tilde{f}_{i}$ are convex, and $\tilde{h}_{i}$ are affine, $(2.24)$ is a convex optimisation problem.

\subsubsection{Semidefinite Program}

Semidefinite program (SDP) refers to an optimisation problem where a linear function is minimised subject to the constraint that an affine combination of symmetric matrices is positive (negative) semidefinite [29, 105, 234, 248]. A SDP can be represented in the form

$$
\begin{array}{cl}
\min _{\mathbf{x}} & \mathbf{c}^{T} \mathbf{x} \\
\text { s.t. } & x_{1} \mathbf{F}_{1}+\ldots+x_{n} \mathbf{F}_{n}+\mathbf{G} \preceq 0, \\
& \mathbf{A x}=\mathbf{b},
\end{array}
$$

where $\mathbf{G}, \mathbf{F}_{1}, \ldots, \mathbf{F}_{n} \in \mathbb{S}^{k}$, and $\mathbf{A} \in \mathbb{R}^{p \times n}$. The inequality in (2.25) is called a linear matrix inequality (LMI).

The SDP reduces to an LP when the matrices $\mathbf{G}, \mathbf{F}_{1}, \ldots, \mathbf{F}_{n}$ are all diagonal since the LMI in (2.25) becomes $n$ linear inequalities.

\subsection{Optimality, Duality and Karush-Kuhn-Tucker (KKT) Conditions}

\subsubsection{Optimality Criterion}

When the objective function $f_{0}$ of the convex optimisation problem (2.14) is differentiable, then using the first-order condition (2.12), a point $\mathbf{x}$ in the domain of 
(2.14) is optimal if and only if [29, p. 139]

$$
\nabla f_{0}(\mathbf{x})^{T}(\mathbf{y}-\mathbf{x}) \geq \mathbf{0}
$$

for all $\mathbf{y}$ in the domain of (2.14).

For an unconstrained problem, the above condition reduces to the well known condition

$$
\nabla f_{0}(\mathbf{x})^{T}(\mathbf{x})=\mathbf{0}
$$

\subsubsection{Duality}

The Lagrangian function of the optimisation problem (2.14) is given by [29, p. 215]

$$
L(\mathbf{x}, \boldsymbol{\lambda}, \boldsymbol{\nu})=f_{0}(\mathbf{x})+\sum_{i=1}^{m} \lambda_{i} f_{i}(\mathbf{x})+\sum_{i=1}^{p} \nu_{i} h_{i}(\mathbf{x})
$$

where $\lambda_{i} \in \mathbb{R}_{+}$is called the Lagrange multiplier associated with the $i$ th inequality constraint $f_{i}(\mathbf{x}) \leq 0$ and $\nu_{i} \in \mathbb{R}$ is called the Lagrange multiplier associated with the $i$ th equality constraint $h_{i}(\mathbf{x})=0$. The vectors $\boldsymbol{\lambda}$ and $\boldsymbol{\nu}$ are also known as dual variables associated with the problem (2.14).

The dual function associated with the problem (2.14) is defined as [29, p. 216]

$$
g(\boldsymbol{\lambda}, \boldsymbol{\nu})=\inf _{\mathbf{x}} L(\mathbf{x}, \boldsymbol{\lambda}, \boldsymbol{\nu})
$$

A useful property of the dual function is that it is concave, even if the original problem (2.14) is not convex. This property arises from the fact that the dual function is a pointwise infimum of a family of affine functions of $(\boldsymbol{\lambda}, \boldsymbol{\nu})$ which is known to be concave.

The dual function gives the lower bound on the optimal value $p^{*}$ of the problem (2.14), i.e., for any $\boldsymbol{\lambda} \succeq \mathbf{0}$ and any $\boldsymbol{\nu}$ we have

$$
g(\boldsymbol{\lambda}, \boldsymbol{\nu}) \leq p^{*}
$$

Using the above property we can find the best lower bound. This can be obtained by solving the following optimisation problem

$$
\begin{array}{ll}
\max _{\boldsymbol{\lambda}, \boldsymbol{\nu}} & g(\boldsymbol{\lambda}, \boldsymbol{\nu}) \\
\text { s.t. } & \boldsymbol{\lambda} \succeq \mathbf{0} .
\end{array}
$$

This problem is known as the dual problem of the problem (2.14). When dealing with the dual problem, the original problem is sometimes referred to as the primal problem. The solution of the dual problem gives the dual optimal variables $\left(\boldsymbol{\lambda}^{*}, \boldsymbol{\nu}^{*}\right)$. 
We denote the optimal value of the dual problem as $d^{*}$. From (2.30), the following inequality follows

$$
d^{*} \leq p^{*}
$$

which holds even if the primal problem is not convex. This is known as weak duality. If (2.32) holds with equality, i.e., $d^{*}=p^{*}$, then strong duality holds.

The difference $p^{*}-d^{*}$ is called the optimal duality gap of the original problem, because it provides the gap between the optimal value of the primal problem and the best lower bound obtained through the dual function. The optimal duality gap is always nonnegative. The duality gap is zero when strict duality holds.

\subsubsection{Karush-Kuhn-Tucker (KKT) Conditions}

If the objective and constraint functions are differentiable and $\mathbf{x}^{*}$ and $\left(\boldsymbol{\lambda}^{*}, \boldsymbol{\nu}^{*}\right)$ are any primal and dual optimum points for which strong duality holds, then the following conditions hold [29, p. 243]

$$
\begin{array}{rlrl}
f_{i}\left(\mathbf{x}^{*}\right) & \leq 0, & i=1, \ldots, m \\
h_{i}\left(\mathbf{x}^{*}\right) & =0, & i=1, \ldots, p \\
\lambda_{i}^{*} \geq 0, & i=1, \ldots, m \\
\lambda_{i}^{*} f_{i}\left(\mathbf{x}^{*}\right) & =0, \quad & i=1, \ldots, m \\
\nabla f_{0}\left(\mathbf{x}^{*}\right)+\sum_{i=1}^{m} \lambda_{i}^{*} \nabla f_{i}\left(\mathbf{x}^{*}\right)+\sum_{i=1}^{p} \nu_{i}^{*} \nabla h_{i}\left(\mathbf{x}^{*}\right) & =0 . &
\end{array}
$$

These conditions are known as Karush-Kuhn-Tucker (KKT) conditions. For any optimisation problem with differentiable objective and constraint functions for which strong duality holds, implies that any pair of primal and dual optimum points must satisfy the KKT conditions [29, p. 244].

When the optimisation problem is convex, the KKT conditions are sufficient for optimality, i.e., if $\tilde{\mathbf{x}}, \tilde{\boldsymbol{\lambda}}$ and $\tilde{\boldsymbol{\nu}}$ are points that satisfy the KKT conditions then $\tilde{\mathbf{x}}$ and $(\tilde{\boldsymbol{\lambda}}, \tilde{\boldsymbol{\nu}})$ are the primal and dual optimum with zero duality gap.

The first two conditions of (2.33) arise due to the fact that at the optimum, the optimisation problem has to be feasible. The third condition arises from the definition of the Lagrange multipliers associated with the inequality constraint functions of the optimisation problem.

The fourth condition is known as the complementary slackness condition [29, p. 242] and is explained as follows. When strong duality holds, it means that

$$
\begin{aligned}
f_{0}\left(\mathbf{x}^{*}\right) & =g\left(\boldsymbol{\lambda}^{*}, \boldsymbol{\nu}^{*}\right) \\
& =f_{0}\left(\mathbf{x}^{*}\right)+\sum_{i=1}^{m} \lambda_{i}^{*} f_{i}\left(\mathbf{x}^{*}\right)+\sum_{i=1}^{p} \nu_{i}^{*} h_{i}\left(\mathbf{x}^{*}\right) .
\end{aligned}
$$


Since $h_{i}\left(\mathbf{x}^{*}\right)=0, i=1, \ldots, p$, for $(2.34)$ to be true, the following must hold

$$
\sum_{i=1}^{m} \lambda_{i}^{*} f_{i}\left(\mathbf{x}^{*}\right)=0 .
$$

Since each term in (2.35) is nonpositive, it implies that

$$
\lambda_{i}^{*} f_{i}\left(\mathbf{x}^{*}\right)=0, \quad i=1, \ldots, m
$$

The last condition of (2.33) states that since $\mathbf{x}^{*}$ minimises $L\left(\mathbf{x}, \boldsymbol{\lambda}^{*}, \boldsymbol{\nu}^{*}\right)$ over $\mathbf{x}$, the gradient of $L\left(\mathbf{x}, \boldsymbol{\lambda}^{*}, \boldsymbol{\nu}^{*}\right)$ must be equal to zero at $\mathbf{x}^{*}$.

The KKT conditions play an important role in optimisation. In some cases it is possible to obtain analytical solutions to the KKT conditions (and hence, to the optimisation problem). In the general case, many algorithms for convex optimisation can be interpreted as methods for solving the KKT conditions.

\subsection{The Epigraph Form}

The epigraph of a function $f: \mathbb{R}^{n} \rightarrow \mathbb{R}$ is defined as $[29$, p. 75$]$

$$
\operatorname{epi} f=\{(\mathbf{x}, t) \mid \mathbf{x} \in \operatorname{dom} f, f(\mathbf{x}) \leq t\}
$$

which is a subset of $\mathbb{R}^{n+1}$. The epigraph provides the link between convex sets and convex functions. A function is convex if and only if its epigraph is a convex set $[29$, p. 75].

Any optimisation problem be restated in an epigraph form. The epigraph form of problem (2.14) is given by [29, p. 134]

$$
\begin{array}{ll}
\min _{\mathbf{x}, t} & t \\
\text { s.t. } & f_{0}(\mathbf{x})-t \leq 0, \\
& f_{i}(\mathbf{x}) \leq 0, \quad i=1 \ldots m \\
& h_{i}(\mathbf{x})=0, \quad i=1 \ldots p
\end{array}
$$

Restating the problem in the epigraph form results in a linear objective function. The main advantage of having a linear objective function is that it simplifies the development of algorithms that solve convex optimisation problems, since an algorithm that solves convex optimisation problems with linear objective can, using the epigraph form transformation, solve any convex optimisation problem [29, p. 134].

Using the epigraph form, some seemingly non-convex problems can be transformed into convex problems. As an example, consider the minimax problem given 
by

$$
\min _{\mathbf{x}} \quad\|\mathbf{A} \mathbf{x}-\mathbf{b}\|_{\infty}=\max \left\{\left|r_{1}\right|, \ldots,\left|r_{m}\right|\right\}
$$

where $\mathbf{A} \in \mathbb{R}^{m \times n}, \mathbf{x} \in \mathbb{R}^{n}, \mathbf{b} \in \mathbb{R}^{m}$ and $r_{i}$ is the $i$ th element of the vector $\mathbf{A x}-\mathbf{b}$. Using the epigraph form, problem (2.39) can be cast into the following LP [29, p. 293]

$$
\begin{array}{ll}
\min _{\mathbf{x}, t} & t \\
\text { s.t. } & -t \mathbf{1} \preceq \mathbf{A} \mathbf{x}-\mathbf{b} \preceq t \mathbf{1},
\end{array}
$$

where $t \in \mathbb{R}$ and $\mathbf{1}$ is an $m$-element vector with all entries equal to one.

\subsection{Convex Relaxations of Non-convex Problems}

Many problems are non-convex and NP-hard, i.e., very difficult to solve. In this section we show how non-convex problems can be relaxed into convex optimisation problems. The NP-hard problems can then be solved efficiently in polynomial time and bounds on the optimal value obtained. In many cases it is also possible to obtain good, but not necessarily optimum, feasible solutions. Two relaxation methods are generally used, direct relaxation and Lagrangian relaxation. In direct relaxation, each non-convex constrained is replaced by a looser convex constraint, while in Lagrangian relaxation, the Lagrangian dual of the non-convex is problem is solved to obtain a lower bound on the optimal value of the non-convex problem [29, p. 653].

Many important engineering problems can be formulated as QCQPs and generally these problems are non-convex and NP-hard. Due to their practical importance, we will focus on the relaxation of non-convex QCQPs. Some of these problems include $[146,147]$ :

- the Boolean least-squares problem in digital communications [153, 228].

- the maximum-likelihood detection problem in multiple-input multiple-output (MIMO) communications [149, 152, 154, 156, 166, 213, 216, 246, 250].

- the MAXCUT problem in network optimisation [89].

- the optimum coded waveform design for radar detection [61].

- the downlink transmit beamforming problem [25, 41, 82, 119, 120, 212].

- the network beamforming problem [99, 100]. 
A non-convex QCQP has the form

$$
\begin{array}{cl}
\min _{\mathbf{x}} & \mathbf{x}^{T} \mathbf{P}_{0} \mathbf{x}+\mathbf{q}_{0}^{T} \mathbf{x}+r_{0} \\
\text { s.t. } & \mathbf{x}^{T} \mathbf{P}_{i} \mathbf{x}+\mathbf{q}_{i}^{T} \mathbf{x}+r_{i} \leq 0, \quad i=1 \ldots m
\end{array}
$$

where $\mathbf{x} \in \mathbb{R}^{n}, \mathbf{P}_{i} \in \mathbb{S}^{n}, i=0,1 \ldots, m, \mathbf{q}_{i} \in \mathbb{R}^{n}, r_{i} \in \mathbb{R}, \mathbf{A} \in \mathbb{R}^{p \times n}$ and $\mathbf{b} \in \mathbb{R}^{p}$. Problem (2.41) is non-convex when at least one of the $\mathbf{P}_{i}$ is not positive semidefinite.

A powerful direct relaxation technique, known as SDP relaxation (SDR) [29, 146], is generally used to relax and solve problems of this nature. This technique will be described in this section.

\subsubsection{Semidefinite Relaxation (SDR)}

SDR makes use of the definition, $\mathbf{X}=\mathbf{x x}^{T}$, to linearise problem (2.41). This definition of $\mathbf{X}$ implies that $\operatorname{rank}(\mathbf{X})=1$. This definition also implies that $\mathbf{x}^{T} \mathbf{P}_{i} \mathbf{x}=$ $\operatorname{tr}\left(\mathbf{P}_{\mathbf{i}} \mathbf{X}\right)$, hence, problem (2.41) can be rewritten as

$$
\begin{array}{ll}
\min _{\mathbf{x}, \mathbf{X}} & \operatorname{tr}\left(\mathbf{P}_{0} \mathbf{X}\right)+\mathbf{q}_{0}^{T} \mathbf{x}+r_{0} \\
\text { s.t. } & \operatorname{tr}\left(\mathbf{P}_{i} \mathbf{X}\right)+\mathbf{q}_{i}^{T} \mathbf{x}+r_{i} \leq 0, \quad i=1 \ldots m \\
& \mathbf{X}=\mathbf{x} \mathbf{x}^{T} .
\end{array}
$$

The constraint $\mathbf{X}=\mathbf{x x}^{T}$ is non-convex constraint; however, it can be relaxed by replacing it with the looser positive semidefinite constraint $\mathbf{X}-\mathbf{x x}^{T} \succeq 0$. With this relaxation, the relaxed problem can be stated as the following SDP

$$
\begin{array}{ll}
\min _{\mathbf{x}, \mathbf{X}} & \operatorname{tr}\left(\mathbf{P}_{0} \mathbf{X}\right)+\mathbf{q}_{0}^{T} \mathbf{x}+r_{0} \\
\text { s.t. } & \operatorname{tr}\left(\mathbf{P}_{i} \mathbf{X}\right)+\mathbf{q}_{i}^{T} \mathbf{x}+r_{i} \leq 0, \quad i=1 \ldots m \\
& \mathbf{X}-\mathbf{x} \mathbf{x}^{T} \succeq 0 .
\end{array}
$$

Utilising the Schur complement [29, p. 650] to represent the last constraint, problem (2.43) can then be rewritten as

$$
\begin{array}{ll}
\min _{\mathbf{x}, \mathbf{X}} & \operatorname{tr}\left(\mathbf{P}_{0} \mathbf{X}\right)+\mathbf{q}_{0}^{T} \mathbf{x}+r_{0} \\
\text { s.t. } & \operatorname{tr}\left(\mathbf{P}_{i} \mathbf{X}\right)+\mathbf{q}_{i}^{T} \mathbf{x}+r_{i} \leq 0, \quad i=1 \ldots m \\
& {\left[\begin{array}{cc}
\mathbf{X} & \mathbf{x} \\
\mathbf{x}^{T} & 1
\end{array}\right] \succeq 0 .}
\end{array}
$$

Problem (2.44) is called the SDP relaxation of the original non-convex problem (2.41). The optimal value of the relaxed problem gives the lower bound on the optimal value of the original non-convex QCQP.

When the objective and constraints of the original problem (2.41) are homoge- 
neous, i.e., there are no linear terms $\mathbf{q}_{i}^{T} \mathbf{x}$, then a simpler relaxed problem can be obtained. The definition, $\mathbf{X}=\mathbf{x x}^{T}$, is again used to linearise the QCQP. In this case, this definition implies that $\mathbf{X} \succeq 0$ and $\operatorname{rank}(\mathbf{X})=1$. Problem (2.41) can then be rewritten as

$$
\begin{array}{cl}
\min _{\mathbf{X}} & \operatorname{tr}\left(\mathbf{P}_{0} \mathbf{X}\right)+r_{0} \\
\text { s.t. } & \operatorname{tr}\left(\mathbf{P}_{i} \mathbf{X}\right)+r_{i} \leq 0, \quad i=1 \ldots m \\
& \mathbf{X} \succeq 0, \\
& \operatorname{rank}(\mathbf{X})=1 .
\end{array}
$$

The rank constraint is the only non-convex constraint in problem (2.45) and the problem can be relaxed by dropping this rank constraint. The resulting SDP is stated as

$$
\begin{array}{ll}
\min _{\mathbf{X}} & \operatorname{tr}\left(\mathbf{P}_{0} \mathbf{X}\right)+r_{0} \\
\text { s.t. } & \operatorname{tr}\left(\mathbf{P}_{i} \mathbf{X}\right)+r_{i} \leq 0, \quad i=1 \ldots m \\
& \mathbf{X} \succeq 0 .
\end{array}
$$

\subsubsection{Lagrangian Relaxation}

Lagrangian relaxation finds a lower bound on the optimal value of the non-convex problem (2.41) by solving the dual of the problem, which is known to be always convex. The Lagrangian of (2.41) is

$$
\begin{aligned}
L(\mathbf{x}, \boldsymbol{\lambda}) & =\mathbf{x}^{T} \mathbf{P}_{0} \mathbf{x}+\mathbf{q}_{0}^{T} \mathbf{x}+r_{0}+\sum_{i=1}^{m} \lambda_{i}\left(\mathbf{x}^{T} \mathbf{P}_{i} \mathbf{x}^{T}+\mathbf{q}_{i}^{T} \mathbf{x}+r_{i}\right) \\
& =\mathbf{x}^{T} \underbrace{\left(\mathbf{P}_{0}+\sum_{i=1}^{m} \lambda_{i} \mathbf{P}_{i}\right)}_{\mathbf{P}} \mathbf{x}+\underbrace{\left(\mathbf{q}_{0}^{T}+\sum_{i=1}^{m} \lambda_{i} \mathbf{q}_{i}^{T}\right)}_{\mathbf{q}} \mathbf{x}+\underbrace{\left(r_{0}+\sum_{i=1}^{m} \lambda_{i} r_{i}\right)}_{r}
\end{aligned}
$$

and the dual function is

$$
\begin{aligned}
g(\boldsymbol{\lambda}) & =\inf _{\mathbf{x}} L(\mathbf{x}, \boldsymbol{\lambda}) \\
& = \begin{cases}r-\frac{1}{4} \mathbf{q}^{T} \mathbf{P}^{\dagger} \mathbf{q}, & \text { if } \mathbf{P} \succeq 0 \text { and } \mathbf{q} \in \mathcal{R}(\mathbf{P}) \\
-\infty, & \text { otherwise. }\end{cases}
\end{aligned}
$$

The dual problem is therefore given by

$$
\begin{array}{ll}
\max _{\boldsymbol{\lambda}} & r-\frac{1}{4} \mathbf{q}^{T} \mathbf{P}^{\dagger} \mathbf{q} \\
\text { s.t. } & \mathbf{P} \succeq 0, \\
& \boldsymbol{\lambda} \succeq 0 .
\end{array}
$$


By introducing an auxiliary variable $\gamma$ to express problem (2.49) in the epigraph form and utilising the Schur complement, problem (2.49) can be rewritten as the following SDP

$$
\begin{array}{ll}
\max _{\boldsymbol{\lambda}, \gamma} & \gamma \\
\text { s.t. } & {\left[\begin{array}{cc}
\mathbf{P} & \frac{1}{2} \mathbf{q} \\
\frac{1}{2} \mathbf{q}^{T} & r-\gamma
\end{array}\right] \succeq 0,} \\
& \boldsymbol{\lambda} \succeq 0 .
\end{array}
$$

It can be shown that problems (2.44) and (2.50) are duals of each other and the bounds obtained are exactly the same.

\subsubsection{Extracting a Rank-1 Solution}

The Lagrangian relaxation method provides a lower bound on the optimal value of the original non-convex QCQP; however, it does not provide a simple way to compute a good feasible point. On the other hand, the SDR method provides both a lower bound and a positive semidefinite matrix, $\mathbf{X}^{*}$, that can be used to extract a good feasible solution.

Note that $\mathbf{X}^{*}$ is not necessarily rank-1 and two methods are generally used to extract a feasible point. The first method chooses the principal eigenvector of $\mathbf{X}^{*}$ projected into the feasible set of the original problem as the solution $\mathbf{x}^{*}$ [146].

The second method, known as Gaussian sampling [146], tends to be more effective and is more common in practise. In this method, a number of random vectors, $\mathbf{x}$, are picked from the Gaussian distribution $\mathcal{N}\left(\tilde{\mathbf{x}}, \mathbf{X}^{*}-\tilde{\mathbf{x}} \tilde{\mathbf{x}}^{T}\right)$, where $\tilde{\mathbf{x}}$ is the solution of problem (2.44), for the non-homogeneous QCQP or from the Gaussian distribution $\mathcal{N}\left(0, \mathbf{X}^{*}\right)$ for the homogeneous QCQP. After sampling enough random vectors, a good approximate solution, $\mathbf{x}^{*}$, is chosen as the random vector that is feasible and results in the minimum value of the objective of the original nonconvex QCQP. This method actually solves the problem "in expectation", i.e., it solves the problem in which statistical expectations are taken of the objective and constraint functions [146].

\subsection{Algorithms For Solving Convex Optimisation Problems}

In a few cases, by utilising the KKT conditions, one can find an analytical solution to the convex optimisation problem (2.14). However, usually no analytical solutions exist and the problem can only be solved using iterative numerical algorithms. In this section we provide a brief review of the numerical algorithms that are 
commonly used to solve convex optimisation problems.

\subsubsection{A Brief History}

The linear program (2.15) is one class of convex optimisation problem for which no analytical solution exists. Dantzig's simplex algorithm (or simplex method) [59] has been used extensively since the 1940s to solve linear programs. The simplex method has been extremely popular despite the fact that it has been shown to have an exponential worst-case complexity [128]. One reason for its popularity was that the theoretical worst-case behaviour never occurs in real world applications. For practical applications, the simplex method was an extremely efficient algorithm with low empirical complexity [171].

Polynomial-time interior-point methods $[62,175]$ for solving convex optimisation problems have recently become very popular. A polynomial-time method has the characteristic that the arithmetic cost of the accuracy of the method is bounded above by a polynomial of the problem size [171]. Interior-point methods for solving non-linear programming problems have been in use for a long time $[64,72,77,78,110,141]$. These methods were applied to solving linear programs; however, they could not compete with the simplex method. They were only seen as theoretical alternatives and not as practical substitutes for the simplex method $[33,37,64,79,177]$. One reason why these methods failed to gain popularity was because they behaved more or less the same as their worst-case complexity bounds and therefore, for practical applications the simplex method was far better even for small problems [171].

In 1984, Karmarkar [121-124] introduced a revolutionary new polynomial-time interior-point method for solving linear programs which claimed to be orders of magnitude more efficient than the simplex method. In practical applications, this method's actual behaviour turned out to be much better than what was predicted by the worst-case theoretical complexity bound. Karmarkar's seminal work laid the foundations for modern interior-point methods and inspired the works of many researchers, see for example, $[4,15,16,91,132,160,167,189]$. Interior-point methods were later extended to handle convex quadratic programs and certain linear complementarity problems [129].

In 1988, Nesterov and Nemirovski [172-175] made an important breakthrough and showed that interior-point methods for linear programming could be generalised to all convex optimisation problems. A key element to their discovery were barrier functions with a property known as self-concordance [173]. To be useful in practise the barrier function must be computable and Nesterov and Nemirovski showed that a self-concordant barrier function exists for every convex set. 


\subsubsection{Unconstrained Minimisation}

An unconstrained optimisation problem takes the form

$$
\min _{\mathbf{x}} f(\mathbf{x})
$$

where $f: \mathbb{R}^{n} \rightarrow \mathbb{R}$ is convex and twice continuously differentiable. We assume that the problem has an optimum point $\mathbf{x}^{*}$ and $f\left(\mathbf{x}^{*}\right)=p^{*}$. In some cases an analytical solution can be obtained by solving the optimality criterion $\nabla f\left(\mathbf{x}^{*}\right)=0$. However, in many cases an iterative algorithm needs to be employed.

An iterative algorithm computes a series of points $\mathbf{x}^{(0)}, \mathbf{x}^{(1)}, \ldots \in \operatorname{dom} f($ known as a minimising sequence) with $f\left(\mathbf{x}^{(k)}\right) \rightarrow p^{*}$ as $k \rightarrow \infty$. The algorithm terminates when $f\left(\mathbf{x}^{(k)}\right)-p^{*} \leq \epsilon$, where $\epsilon>0$ is some specified tolerance.

When $f(\mathbf{x})$ is strongly convex, i.e., there exists an $m>0$ such that

$$
\nabla^{2} f(\mathbf{x}) \succeq m \mathbf{I}
$$

then it can be shown that $[29$, p. 460$]$

$$
p^{*} \geq f(\mathbf{x})-\frac{1}{2 m}\left\|\nabla^{2} f(\mathbf{x})\right\|_{2}^{2}
$$

This inequality can be considered as a condition for suboptimality and can be restated as

$$
\left\|\nabla^{2} f(\mathbf{x})\right\|_{2} \leq(2 m \epsilon)^{\frac{1}{2}} \Longrightarrow f(\mathbf{x})-p^{*} \leq \epsilon
$$

Equation (2.54) implies that when the the gradient is small at a point, then the point is nearly optimal $[29$, p. 460$]$.

For a strongly convex function, the Hessian has an upper bound, i.e., there exists a constant $M$ such that $[29$, p. 460]

$$
\nabla^{2} f(\mathbf{x}) \preceq M \mathbf{I}
$$

and it can be shown that

$$
p^{*} \leq f(\mathbf{x})-\frac{1}{2 M}\left\|\nabla^{2} f(\mathbf{x})\right\|_{2}^{2}
$$

The constants $m$ and $M$ play an important role in deriving the complexity estimates of iterative algorithms for solving convex optimisation problems. However, these constants are rarely known and the inequality (2.54) cannot be used as a practical terminating criterion. 


\subsubsection{Descent Algorithms}

Generally, descent algorithms produce the minimising sequence $\mathbf{x}^{(k)}, k=1, \ldots$, where $[29$, p. 463]

$$
\mathbf{x}^{(k+1)}=\mathbf{x}^{(k)}+t^{(k)} \Delta \mathbf{x}^{(k)}
$$

where $t^{(k)}>0$ is known as the step size and $\Delta \mathbf{x}^{(k)}$ is called the search or descent direction. On each iteration the next point $\mathbf{x}^{(k+1)}$ is calculated such that $f\left(\mathbf{x}^{(k+1)}\right)<$ $f\left(\mathbf{x}^{(k)}\right)$.

The structure of a general descent algorithm is shown in Algorithm 1.

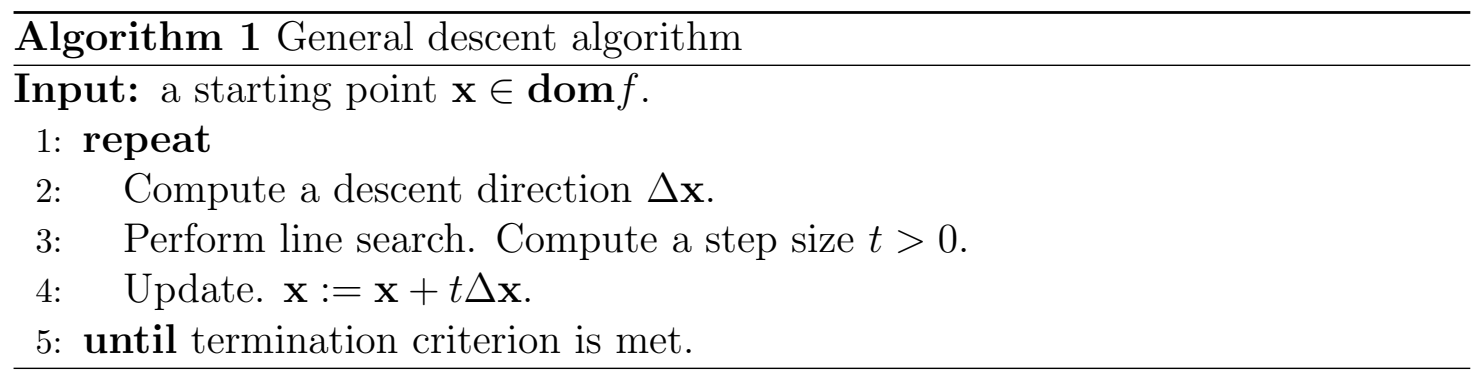

\section{Line Search}

The second step in a general descent algorithm is called a line search which involves finding a the step size, $t$, along the line $\left\{\mathbf{x}+t \Delta \mathbf{x} \mid t \in \mathbb{R}_{+}\right\}$. The aim is to find a $t$ that minimises $f$ along the line $\left\{\mathbf{x}+t \Delta \mathbf{x} \mid t \in \mathbb{R}_{+}\right\}$. Line searches can be either exact or inexact [29, p. 464]. Practical line searches are almost always inexact. Inexact line searches seek to approximately minimise or just reduce $f$. The backtracking line search is an example of a commonly used inexact line search [29, p. 464].

\section{Descent Direction}

The descent direction is chosen to satisfy the following condition

$$
\nabla f\left(\mathbf{x}^{(k)}\right)^{T} \Delta \mathbf{x}^{(k)}<0
$$

This condition follows directly from the optimality criterion (2.26) and ensures that $f\left(\mathbf{x}^{(k+1)}\right)<f\left(\mathbf{x}^{(k)}\right)$. Gradient descent, steepest descent and Newton's method are the three commonly used methods for calculating the descent direction [29, p. 463].

The gradient descent method uses the negative gradient as its descent direction, i.e, $\Delta \mathbf{x}=-\nabla f(\mathbf{x})$. The algorithm terminates when the gradient becomes sufficiently small.

The steepest descent method chooses the descent direction as [29, p. 475]

$$
\Delta \mathbf{x}_{\mathrm{nsd}}=\operatorname{argmin}\left\{\nabla f(\mathbf{x})^{T} \mathbf{v} \mid\|\mathbf{v}\| \leq 1\right\}
$$


This is the direction that makes $\nabla f(\mathbf{x})^{T} \Delta \mathbf{x}_{\mathrm{nsd}}$ the most negative. $\|\cdot\|$ can be any norm on $\mathbb{R}^{n}$. Commonly used norms are the Euclidean norm, the quadratic norm and the $\ell_{1}$ norm. The steepest descent direction becomes the negative gradient when the Euclidean norm is used. Hence, the steepest descent method can be seen as a general form of the gradient descent method. The steepest descent method terminates when the gradient becomes sufficiently small.

Newton's method uses the following descent direction (known as the Newton step) $[29$, p. 484]

$$
\Delta \mathbf{x}_{\mathrm{nt}}=-\nabla^{2} f(\mathbf{x})^{-1} \nabla f(\mathbf{x})
$$

The Newton step is both the minimiser of the second-order Taylor approximation of $f$ and the steepest descent direction when a quadratic norm defined by the Hessian is used. A quantity known as the Newton decrement at $\mathbf{x}$ and defined as

$$
\mu(\mathbf{x})=\left(\nabla f(\mathbf{x})^{T} \nabla^{2} f(\mathbf{x})^{-1} \nabla f(\mathbf{x})\right)^{\frac{1}{2}}
$$

is generally used to determine the termination criterion for Newton's method. The algorithm is terminated when $\frac{\mu(\mathbf{x})^{2}}{2} \leq \epsilon$.

\section{Convergence of Descent Methods}

In this section we summarise the convergence of the three descent methods described above. Convergence is defined as the rate at which the error $f\left(\mathbf{x}^{(k)}\right)-p^{*}$ converges to zero. A detailed convergence analysis is beyond the scope of this work. Detailed analysis can be found in [29, 172-175].

The gradient descent method has approximately linear convergence. The convergence rate is dependent upon the constants $m$ and $M$ described in Section 2.8.2, the parameters used for the backtracking line search and the condition number of the Hessian of $f(\mathbf{x})$. Simplicity is the main advantage of the gradient method. Its main disadvantage is that the convergence rate depends greatly on the condition number of the Hessian; the gradient method becomes extremely slow for practical use when the condition number is large.

The steepest descent method also has approximately linear convergence. The convergence rate is dependent upon the constants $m$ and $M$, the parameters used for the backtracking line search and the norm used. The norm used determines the resulting condition number of the Hessian of the transformed problem and this has a critical effect on the convergence rate of the problem. Choosing the norm can be a difficult task. For example, when the quadratic norm is used, it requires choosing a matrix for the norm calculation and there can be a large number of matrices to choose from. The steepest descent method will work very poorly for some of these choices and very well when a good matrix can be identified. 
Newton's method has a very rapid convergence; quadratic near $\mathbf{x}^{*}$. The convergence rate is only dependent on the constants $m$ and $M$ and the parameters used for the backtracking line search. The main advantage of Newton's method is that the convergence rate does not depend on condition number of the Hessian of the objective. The main disadvantage is that this method requires the computation of the Hessian which can be computationally expensive.

\subsubsection{Self-concordance}

The idea of a self-concordance function was introduced in 1988 by Nesterov and Nemirovski [172-175]. A convex function $f: \mathbb{R} \rightarrow \mathbb{R}$ is self-concordant if

$$
\left|f^{\prime \prime \prime}(x)\right| \leq 2 f^{\prime \prime}(x)^{\frac{3}{2}}
$$

for all $x \in \operatorname{dom} f$. A function $f: \mathbb{R}^{n} \rightarrow \mathbb{R}$ is self-concordant if it is self-concordant when restricted to a line, i.e., if the function $\hat{f}(t)=f(\mathbf{x}+t v)$ is a self-concordant function of $t$ for all $x \in \operatorname{dom} f$ and for all $v$.

As mentioned in Section 2.8.3, the convergence rate of the descent methods depends on the constants $m$ and $M$. However, in practise, these constants are usually not known, hence, the number of iterations required for the algorithms are not generally known. Self-concordant functions, on the other hand, do not suffer from this problem. For example, the complexity of Newtown's method when applied to self-concordant functions does not depend on any unknown constants. It only depends on the parameters for the backtracking line search and the accuracy $\epsilon$. Another advantage of self-concordant functions is that they are affine-invariant, hence, the complexity estimate obtained for Newton's method applied to a selfconcordant function is independent of affine changes of coordinates [29, 172-175].

The logarithmic barrier function used in interior-point methods is an example of a self-concordant function.

\subsubsection{Equality Constrained Minimisation}

Equality constrained minimisation problems take the form $[29$, p. 521]

$$
\begin{array}{cl}
\min _{\mathbf{x}} & f(\mathbf{x}) \\
\text { s.t. } & \mathbf{A x}=\mathbf{b} .
\end{array}
$$

We assume that $f: \mathbb{R}^{n} \rightarrow \mathbb{R}$ is convex and twice continuously differentiable, and $\mathbf{A} \in \mathbb{R}^{p \times n}$ with rank $\mathbf{A}=p<n$.

Below we describe how Newton's method can be modified to handle problems of this nature. The objective of problem (2.63) can be approximated near a feasible point $\mathbf{x}$ with its second-order Taylor approximation near $\mathbf{x}$ and the problem restated 
as

$$
\begin{array}{ll}
\min _{\mathbf{v}} & f(\mathbf{x})+\nabla f(\mathbf{x})^{T} \mathbf{v}+\frac{1}{2} \mathbf{v}^{T} \nabla^{2} f(\mathbf{x}) \mathbf{v} \\
\text { s.t. } & \mathbf{A}(\mathbf{x}+\mathbf{v})=\mathbf{b},
\end{array}
$$

with variable v. Problem (2.64) is a convex quadratic minimisation problem with equality constraints, and the KKT conditions can be used to solve it analytically. Using the second and fifth KKT conditions from (2.33), the optimality conditions for problem (2.64) are

$$
\mathbf{A}\left(\mathbf{x}+\mathbf{v}^{*}\right)=\mathbf{b}, \quad \nabla f(\mathbf{x})+\nabla^{2} f(\mathbf{x}) \mathbf{v}^{*}+\mathbf{w}^{* T} \mathbf{A} \mathbf{v}=0
$$

where $\mathbf{w}$ is the dual variable associated with the equality constraint. From the optimality conditions above, it is clear that $\mathbf{A v}^{*}=0$ and this ensures that a feasible solution is obtained on every step. In the modified Newton's method, we choose $\Delta \mathbf{x}_{\mathrm{nt}}=\mathbf{v}^{*}$, i.e., the Newton step is what must be added to $\mathbf{x}$ to solve the problem when the quadratic approximation is used [29, p. 526]. The optimality conditions can be compactly written as

$$
\left[\begin{array}{cc}
\nabla^{2} f(\mathbf{x}) & \mathbf{A}^{T} \\
\mathbf{A} & 0
\end{array}\right]\left[\begin{array}{c}
\Delta \mathbf{x}_{\mathrm{nt}} \\
\mathbf{w}^{*}
\end{array}\right]=\left[\begin{array}{c}
-\nabla f(\mathbf{x}) \\
0
\end{array}\right] .
$$

The matrix on the left hand side of (2.65) is known as the KKT matrix. A solution to (2.65) only exists when the KKT matrix is nonsingular.

To summarise, equality constrained minimisation problems can be solved using Newton's method by analytically solving a series of convex quadratic minimisation problem with equality constraints that approximate the original problem.

\subsubsection{Interior-point Methods}

In this section, we briefly discuss an interior-point method, known as the barrier method, for solving convex optimisation minimisation problems with both equality and inequality constraints, i.e., problems of the form

$$
\begin{array}{cl}
\min _{\mathbf{x}} & f_{0}(\mathbf{x}) \\
\text { s.t. } & f_{i}(\mathbf{x}) \leq 0, \quad i=1 \ldots m \\
& \mathbf{A x}=\mathbf{b},
\end{array}
$$

where $f_{0}, \ldots, f_{m}: \mathbb{R}^{n} \rightarrow \mathbb{R}$ are convex and twice continuously differentiable, and $\mathbf{A} \in \mathbb{R}^{p \times n}$ with rank $\mathbf{A}=p<n$. The assumption is that the problem is strictly feasible, i.e., there exists an $\mathbf{x}$ in the domain of the problem that satisfies $\mathbf{A x}=\mathbf{b}$ and $f_{i}(\mathbf{x})<0$ for $i=1, \ldots, m$. This assumption implies that strict duality holds, 
i.e., there exist primal and dual optimal points, $\mathbf{x}^{*}, \lambda^{*} \in \mathbb{R}^{m}$ and $\nu^{*} \in \mathbb{R}^{p}$ that satisfy the KKT conditions (2.33).

\section{The Barrier Method}

The aim of the barrier method is to formulate the inequality constrained problem (2.66) as an equality constrained problem to which the method discussed in Section 2.8.5 can be applied. Problem (2.66) can be converted into an equality constrained problem by including the inequality constraint functions into the objective with the use of an appropriate barrier function. Problem (2.66) can then be restated as $[29$, p. 561$]$

$$
\begin{array}{ll}
\min _{\mathbf{x}} & f_{0}(\mathbf{x})+\sum_{i=1}^{m} I_{-}\left(f_{i}(\mathbf{x})\right) \\
\text { s.t. } & \mathbf{A x}=\mathbf{b},
\end{array}
$$

where $I_{-}: \mathbb{R} \rightarrow \mathbb{R}$ is the barrier function. The barrier function acts like a penalty function, applying an infinite penalty when its argument is positive and no penalty when the argument is non-positive. The barrier function also needs to be differentiable so that Newton's method can be applied.

In practice, a logarithmic barrier function is generally used to approximate the ideal barrier function. The function is defined as

$$
I_{-}(u)=-\frac{1}{t} \log (-u)
$$

where $t>0$ sets the accuracy of the approximation. Larger values of $t$ give better approximation accuracy; but also make the objective of problem (2.67) difficult to minimise [29, p. 563]. In practice, the problem (2.66) is solved by solving a series of problems of the form (2.67), and $t$ is increased at each step.

Problem (2.67) can be re-written as

$$
\begin{array}{ll}
\min _{\mathbf{x}} & f_{0}(\mathbf{x})-\sum_{i=1}^{m} \log \left(-f_{i}(\mathbf{x})\right) \\
\text { s.t. } & \mathbf{A x}=\mathbf{b},
\end{array}
$$

with no change to the optimum point. On every step, problem (2.69) is solved using Newton's method for equality constrained problems with the solution of the previous step as the starting point for the next. The set of solutions, $\mathbf{x}^{*}(t)$, are known as the central points, and the path that these solutions follow is called the central path of problem (2.66). The central points are strictly feasible (in the interior of the feasible region), i.e., $f_{i}\left(\mathbf{x}^{*}(t)\right)<0, i=1, \ldots, m$ and satisfy the KKT 
conditions

$$
\begin{aligned}
\mathbf{A x}^{*}(t) & =\mathbf{b} \\
t \nabla f_{0}\left(\mathbf{x}^{*}(t)\right)+\sum_{i=1}^{m} \frac{1}{-f_{i}\left(\mathbf{x}^{*}(t)\right)} \nabla f_{i}\left(\mathbf{x}^{*}(t)\right)+\mathbf{A}^{T} \hat{\nu} & =0
\end{aligned}
$$

where $\hat{\nu}$ is the dual optimal point associated with the equality constraint of problem (2.69). Using the definitions

$$
\lambda_{i}^{*}(t)=-\frac{1}{t f_{i}\left(\mathbf{x}^{*}(t)\right)}, i=1, \ldots, m \quad \nu^{*}=\frac{\hat{\nu}}{t},
$$

(2.70b) can be re-stated as

$$
\nabla f_{0}\left(\mathbf{x}^{*}(t)\right)+\sum_{i=1}^{m} \lambda_{i}^{*}(t) \nabla f_{i}\left(\mathbf{x}^{*}(t)\right)+\mathbf{A}^{T} \nu^{*}=0
$$

Equation (2.71) is seen to be the KKT condition that the gradient of the Lagrangian of the original problem (2.66) vanishes at $\mathbf{x}^{*}(t)$, i.e., $\mathbf{x}^{*}(t)$ is the minimiser of the Lagrangian of the original problem. Hence, $\lambda^{*}(t)$ and $\nu^{*}$ are the dual feasible pair of the original problem. The dual function of the original problem can be shown to be

$$
g\left(\lambda^{*}(t), \nu^{*}\right)=f_{0}\left(\mathbf{x}^{*}(t)\right)-\frac{m}{t}
$$

which gives the suboptimality of the original problem at the current central point. $\frac{m}{t}$ is the duality gap and is used as the termination criterion for the barrier method.

Algorithm 2 shows the general structure of the barrier method. It consists of two nested iterations. The outer iteration increases the $t$ parameter. The inner iteration is the Newton's method used to solve problem (2.69). The $\mu$ and $t^{(0)}$

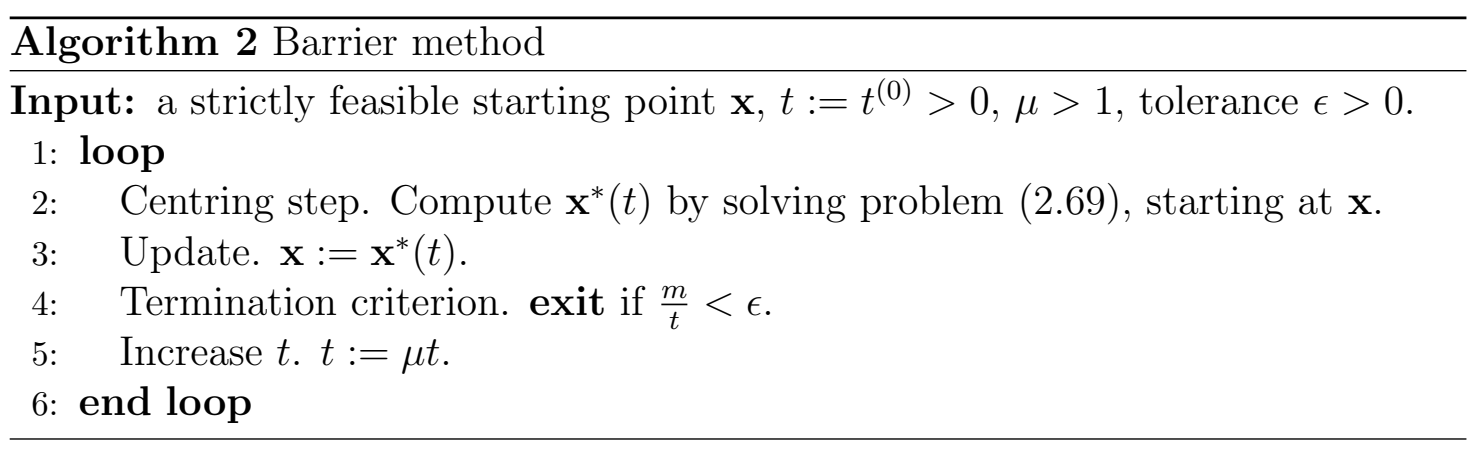

parameters need to be chosen carefully as they influence the number of iterations required to solve the optimisation problem. General guidelines on how to choose these parameters are available in [29, p. 569]. 


\section{Feasible Starting Point}

As seen in Algorithm 2, the barrier method requires a feasible starting point $\mathbf{x}$. When such a point is not known, the barrier method is preceded by a preliminary stage, called phase I, in which a strictly feasible point is computed or it is found that the constraints are infeasible [29, p. 579]. One basic method of doing this is to solve the following optimisation problem [29, p. 579]

$$
\begin{array}{ll}
\min _{\mathbf{x}, s} & s \\
\text { s.t. } & f_{i}(\mathbf{x}) \leq s, \quad i=1 \ldots m, \\
& \mathbf{A x}=\mathbf{b}
\end{array}
$$

where $s \in \mathbb{R}$. The variable $s$ can be considered as the bound on the maximum infeasibility of the inequalities, and the goal is to push the maximum infeasibility below zero. Problem (2.73) is always strictly feasible, since a point $\mathbf{x} \in \operatorname{dom} f_{1} \cap$ $\ldots \cap \operatorname{dom} f_{m}$, with $\mathbf{A x}=\mathbf{b}$ can be chosen as a feasible starting point and $s$ can be chosen to be any number larger than $\max _{i=1, \ldots, m} f_{i}(\mathbf{x})[29$, p. 579]. Hence, problem (2.73) can itself be solved using the barrier method. The constraints have a strictly feasible solution if the optimum value of problem (2.73) is less than zero, and are infeasible if the optimum value is greater than zero. If the optimum value is equal to zero and the minimum is attained at $\mathbf{x}^{*}$ and $s^{*}=0$, then the set of inequalities is feasible, but not strictly feasible [29, p. 579].

A variation of the above method, known as sum of infeasibilities, can also be used as a phase I method. This method minimises the sum of the infeasibilities. The following optimisation problem is used [29, p. 580]

$$
\begin{array}{ll}
\min _{\mathbf{x}, s} & \mathbf{1}^{\mathrm{T}} \mathbf{s} \\
\text { s.t. } & f_{i}(\mathbf{x}) \leq s_{i}, \quad i=1 \ldots m, \\
& \mathbf{A x}=\mathbf{b} \\
& \mathbf{s} \succeq 0
\end{array}
$$

where $\mathbf{1}$ is a vector of length $m$ with all entries equal to one and $\mathbf{s} \in \mathbb{R}_{++}^{m}$. For fixed $\mathbf{x}$, the optimal value of $s_{i}$ is $\max \left\{f_{i}(\mathbf{x}), 0\right\}$, so in this problem the sum of the infeasibilities is being minimised. The optimal value of problem (2.74) is zero and achieved if and only if the original set of inequalities and inequalities is feasible [29, p. 580]. 


\subsection{Summary}

Convex optimisation theory and techniques used in the majority of this thesis has been presented. The concepts of convex sets, convex functions and convex optimisation problems have been introduced. A review of Lagrange duality and the well known KKT conditions for optimality has been provided. The epigraph form which provides the link between convex sets and convex functions and allows seemingly non-convex problems to be transformed into convex problems has been discussed. A powerful technique known as convex relaxation, which allows non-convex problems to be relaxed into convex problems was reviewed. Finally, algorithms for solving convex optimisation problems have been discussed. 


\section{Chapter 3}

\section{Robust Optimisation}

Robust optimisation deals with optimisation problems in which the input data has some degree of uncertainty. The uncertainty may arise due to noise, measurement errors or partial knowledge of the data. This occurs frequently in many real world engineering problems and robust optimisation methods seek to find solutions that offer robustness against the uncertainty. As demonstrated in [21], solutions to many optimisation problems suffer from sensitivity to uncertain data and even minor uncertainties can render the problems suboptimal or even infeasible. Robust optimisation methods can be broadly classed into two categories, namely, methods that use bounded uncertainty set models [19-23, 26, 66, 67, 218] and those that utilise the statistical knowledge of data [28] to develop robust solutions.

Bounded uncertainty (also known as worst-case) based robust optimisation is generally used when hard performance guarantees are sought, i.e., constraints must be satisfied for all realisations of the data within some predefined uncertainty set $\mathcal{U}$. Generally, no underlying stochastic model for the data is assumed; however, any such knowledge can be used to form the uncertainty set, for instance, the ellipsoidal uncertainty set [19] naturally arises when data has a normal distribution.

The stochastic programming (SP) method is utilised when the knowledge of an underlying stochastic model for the uncertain data exists and constraint violations with some probability can be tolerated, for example, probability of outage in communication systems $[38,117]$. The probability based constraints that occur in SP are generally referred to as soft constraints.

In this chapter, we discuss the bounded uncertainty and stochastic based robust optimisation methods and their applications to communication systems. 


\subsection{Bounded Uncertainty Based Robust Optimi- sation}

A general bounded uncertainty based robust optimisation problem is stated as

$$
\begin{array}{cl}
\min _{\mathbf{x}} & f_{0}(\mathbf{x}) \\
\text { s.t. } & f_{i}\left(\mathbf{x}, \mathbf{u}_{i}\right) \leq 0, \quad \forall \mathbf{u}_{i} \in \mathcal{U}_{i}, i=1 \ldots l
\end{array}
$$

where $\mathbf{x} \in \mathbb{R}^{n}$ is the optimisation variable, $f_{0}, f_{i}: \mathbb{R}^{n} \rightarrow \mathbb{R}$ are functions and $\mathbf{u}_{i} \in \mathbb{R}^{k}$ is the uncertain data that is known to belong to the set $\mathcal{U}_{i}$. The aim of (3.1) is to find the $\mathbf{x}^{*}$ that minimises $f_{0}$ for all realisations of $\mathbf{u}_{i}$ within $\mathcal{U}_{i}$. Generally $\mathcal{U}_{i}$ are continuous sets, therefore, (3.1) has an infinite number of constraints. (3.1) is known as a semi-infinite optimisation problem [19] and is known to be computationally intractable [20]. However, some geometries of $\mathcal{U}_{i}$ exist that lead to computationally tractable solutions. These include the ellipsoidal uncertainty set $[19,20,66,67]$ and the polyhedral and the cardinality constrained uncertainty sets [26]. The ellipsoidal uncertainty model is the most widely used set in the literature.

\subsubsection{Ellipsoidal Uncertainty}

The ellipsoidal uncertainty model is the most commonly used model for representing uncertainty. This model has many advantages, some of which include:

- An ellipsoid has a simple mathematical representation given by (2.7) and can be easily numerically handled.

- Ellipsoids and intersections of ellipsoids can be used to represent more complicated uncertainty sets [19].

- In many cases where the uncertain data has an underlying stochastic model, the stochastic uncertainty can be replaced by a deterministic ellipsoidal uncertainty where the ellipsoid is represented using the mean and covariance matrix of the uncertain data [19]. Fig. 3.1 shows points drawn from a bivariate normal distribution and three bounding ellipsoids.

\section{Linear Optimisation with Ellipsoidal Uncertainty}

Consider the following robust LP with uncertain data $\mathbf{G}$ and $\mathbf{h}$ that belong to the uncertainty set $\mathcal{U}$.

$$
\begin{array}{ll}
\min _{\mathbf{x}} & \mathbf{c}^{T} \mathbf{x} \\
\text { s.t. } & \mathbf{G x} \preceq \mathbf{h}, \quad \forall(\mathbf{G}, \mathbf{h}) \in \mathcal{U} .
\end{array}
$$




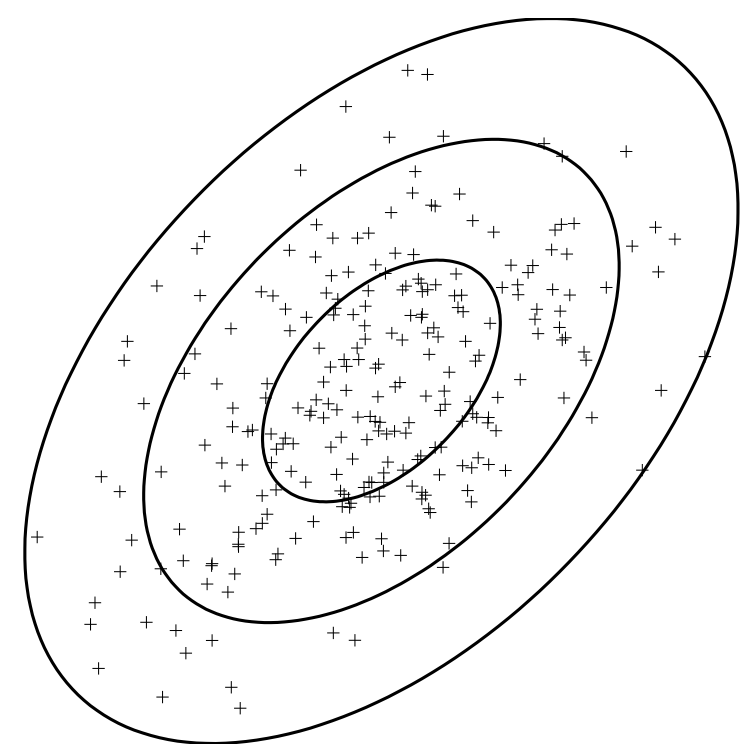

Figure 3.1: Points drawn from a bivariate normal distribution and three bounding ellipsoids calculated from mean and covariance matrix of data.

By defining $\tilde{\mathbf{x}}=\left[\mathbf{x}^{T} 1\right]^{T}, \tilde{\mathbf{c}}=\left[\mathbf{c}^{T} 0\right]^{T}, \tilde{\mathbf{G}}=[\mathbf{G}-\mathbf{h}]$ and $\tilde{\mathbf{a}}=\left[\begin{array}{lll}0 & \ldots & 0\end{array}\right]^{T}$, problem (3.2) can be restated as

$$
\begin{array}{cl}
\min _{\tilde{\mathbf{x}}} & \tilde{\mathbf{c}}^{T} \tilde{\mathbf{x}} \\
\text { s.t. } & \tilde{\mathbf{g}}_{i}^{T} \tilde{\mathbf{x}}_{i} \leq 0, \quad \forall \tilde{\mathbf{g}}_{i} \in \mathcal{U}_{i}, i=1 \ldots l \\
& \tilde{\mathbf{a}}^{T} \tilde{\mathbf{x}}=1,
\end{array}
$$

where $\tilde{\mathbf{g}}_{i}$ is the $i$ th row of $\tilde{\mathbf{G}}$ and we have assumed constraint-wise uncertainty, i.e., the uncertainty set $\mathcal{U}$ is the direct product of the partial uncertainty sets $\mathcal{U}_{i}$ [20]. For ellipsoidal uncertainty, $\mathcal{U}_{i}$ is given by

$$
\mathcal{U}_{i}=\left\{\tilde{\mathbf{g}}_{i}=\tilde{\mathbf{g}}_{c, i}+\mathbf{A}_{i} \mathbf{u}_{i} \mid\left\|\mathbf{u}_{i}\right\|_{2} \leq 1\right\}
$$

where $\tilde{\mathbf{g}}_{c, i}$ is the nominal value of $\tilde{\mathbf{g}}_{i}$ which defines the center of the $i$ th ellipsoid.

We see that the $i$ th robust constraint, $\tilde{\mathbf{g}}_{i}^{T} \tilde{\mathbf{x}}_{i} \leq 0$, can only be satisfied for all $\tilde{\mathrm{g}} \in \mathcal{U}_{i}$, if and only if

$$
\max _{\tilde{\mathbf{g}}_{i} \in \mathcal{U}_{i}}\left\{\tilde{\mathbf{g}}_{i}^{T} \tilde{\mathbf{x}}_{i}\right\} \leq 0, \quad \text { s.t. }\left\|\mathbf{u}_{i}\right\|_{2} \leq 1
$$

It is easily verified that $\tilde{\mathbf{g}}_{i}^{T} \tilde{\mathbf{x}}_{i}$ is maximised when $\mathbf{u}_{i}=\frac{\mathbf{A}_{i}^{T} \tilde{\mathbf{x}}}{\| \mathbf{A}_{i}^{T} \tilde{\mathbf{x}}_{2}}$. The $i$ th robust constraint can therefore be stated as

$$
\left\|\mathbf{A}_{i}^{T} \tilde{\mathbf{x}}\right\|_{2} \leq-\tilde{\mathbf{g}}_{c, i}^{T} \tilde{\mathbf{x}}
$$


and problem (3.3) as

$$
\begin{array}{ll}
\min _{\tilde{\mathbf{x}}} & \tilde{\mathbf{c}}^{T} \tilde{\mathbf{x}} \\
\text { s.t. } & \left\|\mathbf{A}_{i}^{T} \tilde{\mathbf{x}}\right\|_{2} \leq-\tilde{\mathbf{g}}_{c, i}^{T} \tilde{\mathbf{x}}, \quad i=1 \ldots l \\
& \tilde{\mathbf{a}}^{T} \tilde{\mathbf{x}}=1,
\end{array}
$$

which is recognised as a SOCP that can be solved efficiently using interior point methods. Hence, the ellipsoidal uncertainty model has enabled a computationally intractable semi-infinite problem to be transformed into a convex optimisation problem that can be solved in polynomial time.

\section{Quadratic and Semidefinite Optimisation with Ellipsoidal Uncertainty}

In [19], it was shown that QCQPs and SOCPs with ellipsoidal uncertainty can be transformed into robust SDPs. When the uncertainty is an intersection of ellipsoids, the robust formulation turns out to be a NP-hard problem.

The robust counterparts of SDPs with ellipsoidal uncertainty are generally NPhard problems [19].

\subsubsection{The S-Procedure}

The S-procedure $[76,249]$ is commonly used in system theory to derive stability and performance results for nonlinear and uncertain systems. It began to be commonly used in robust control research after the advancements of [161]. In robust optimisation, it is generally used to transform a semi-infinite problem into a convex problem when there is one quadratic objective subject to one quadratic constraint.

The S-procedure is stated as follows. Let $f_{i}: \mathbb{R}^{n} \rightarrow \mathbb{R}, i=0, \ldots, m$ be real valued functions, $s_{i} \geq 0, i=1, \ldots, m$ be real numbers and consider the following two conditions

$$
\begin{aligned}
& f_{0}(\mathbf{x}) \geq 0, \forall \mathbf{x} \in \mathbb{R}^{n} \mid f_{i}(\mathbf{x}) \geq 0, i=1, \ldots, m \\
& \exists_{s_{i} \geq 0, i=1, \ldots, m} \mid f_{0}(\mathbf{x})-\sum_{i=1}^{m} s_{i} f_{i}(\mathbf{x}) \geq 0, \forall \mathbf{x} \in \mathbb{R}^{n} .
\end{aligned}
$$

It is obvious that (3.9) implies (3.8), i.e., (3.8) and (3.9) are equivalent. In this case, the S-procedure is called lossless for the inequality $f_{0}(\mathbf{x}) \geq 0$, subject to the constraints $f_{i}(\mathbf{x}) \geq 0, i=1, \ldots, m[249]$. Therefore, the S-procedure is a method of verifying (3.8) using (3.9). (3.9) is usually easier to verify than (3.8).

The S-procedure is applied to optimisation problems involving quadratic functions as follows. Let $f_{i}(\mathbf{x})=\mathbf{x}^{T} \mathbf{P}_{i} \mathbf{x}+\mathbf{q}_{i}^{T} \mathbf{x}+r_{i}, i=1, \ldots, m$, where $\mathbf{x} \in \mathbb{R}^{n}$, $\mathbf{P}_{i} \in \mathbb{R}^{n \times n}, \mathbf{q}_{i} \in \mathbb{R}^{n}$ and $r_{i} \in \mathbb{R} . \mathbf{P}_{i}$ is not assumed to be positive semidefinite, hence $f_{i}$ is not necessarily convex. The aim is to verify (3.8), a semi-infinite NP 
hard problem, using (3.9). In this scenario, (3.9) can be written as

$$
\begin{aligned}
& \exists_{s_{i} \geq 0} \mid \mathbf{x}^{T} \mathbf{P}_{0} \mathbf{x}+\mathbf{q}_{0}^{T} \mathbf{x}+r_{0}-\sum_{i=1}^{m} s_{i}\left(\mathbf{x}^{T} \mathbf{P}_{i} \mathbf{x}+\mathbf{q}_{i}^{T} \mathbf{x}+r_{i}\right) \geq 0, \forall \mathbf{x} \in \mathbb{R}^{n}, \\
& \exists_{s_{i} \geq 0} \mid\left[\begin{array}{c}
\mathbf{x} \\
1
\end{array}\right]^{T}\left[\begin{array}{cc}
\mathbf{P}_{0}+\sum_{i=1}^{m} s_{i} \mathbf{P}_{i} & \frac{1}{2}\left(\mathbf{q}_{0}+\sum_{i=1}^{m} s_{i} \mathbf{q}_{i}\right) \\
\frac{1}{2}\left(\mathbf{q}_{0}+\sum_{i=1}^{m} s_{i} \mathbf{q}_{i}\right)^{T} & r_{0}+\sum_{i=1}^{m} s_{i} r_{i}
\end{array}\right]\left[\begin{array}{c}
\mathbf{x} \\
1
\end{array}\right] \geq 0, \forall \mathbf{x} \in \mathbb{R}^{n}, \\
& \exists_{s_{i} \geq 0} \mid\left[\begin{array}{cc}
\mathbf{P}_{0}+\sum_{i=1}^{m} s_{i} \mathbf{P}_{i} & \frac{1}{2}\left(\mathbf{q}_{0}+\sum_{i=1}^{m} s_{i} \mathbf{q}_{i}\right) \\
\frac{1}{2}\left(\mathbf{q}_{0}+\sum_{i=1}^{m} s_{i} \mathbf{q}_{i}\right)^{T} & r_{0}+\sum_{i=1}^{m} s_{i} r_{i}
\end{array}\right] \succeq 0 .
\end{aligned}
$$

As demonstrated above, the S-procedure has allowed a semi-infinite NP hard problem to be transformed into a LMI.

The following example illustrates the use of the S-procedure in uncertain optimisation problems. Consider the following uncertain linear inequality where both a and $b$ have some bounded uncertainty on them.

$$
(\mathbf{a}+\Delta \mathbf{a})^{T} \mathbf{x} \leq(b+\Delta b), \forall\|\Delta \mathbf{a}\|_{2} \leq \epsilon, \forall|\Delta b| \leq \epsilon
$$

By defining $\mathbf{e}=\left[\Delta \mathbf{a}^{T} / \epsilon \Delta b / \epsilon\right]^{T},(3.11)$ can be expressed as

$$
b-\mathbf{a}^{T} \mathbf{x}+\epsilon\left[-\mathbf{x}^{T} 1\right] \mathbf{e} \geq 0, \forall\|\mathbf{e}\|_{2} \leq 2 .
$$

Applying the S-procedure to (3.12), we obtain

$$
\begin{aligned}
& \exists_{s \geq 0} \mid b-\mathbf{a}^{T} \mathbf{x}+\epsilon\left[-\mathbf{x}^{T} 1\right] \mathbf{e}-s\left(2-\mathbf{e}^{T} \mathbf{e}\right) \geq 0, \forall \mathbf{e} \in \mathbb{R}^{n+1}, \\
& \exists_{s \geq 0} \mid\left[\begin{array}{l}
\mathbf{e} \\
1
\end{array}\right]^{T}\left[\begin{array}{rr}
s \mathbf{I} & \frac{\epsilon}{2}\left[\begin{array}{c}
-\mathbf{x} \\
1
\end{array}\right] \\
\frac{\epsilon}{2}\left[\begin{array}{ll}
-\mathbf{x}^{T} & 1
\end{array}\right] & b-\mathbf{a}^{T} \mathbf{x}-2 s
\end{array}\right]\left[\begin{array}{l}
\mathbf{e} \\
1
\end{array}\right] \geq 0, \forall \mathbf{e} \in \mathbb{R}^{n+1}, \\
& \exists_{s \geq 0} \mid\left[\begin{array}{cc}
s \mathbf{I} & \frac{\epsilon}{2}\left[\begin{array}{c}
-\mathbf{x} \\
1
\end{array}\right] \\
\frac{\epsilon}{2}\left[\begin{array}{cc}
-\mathbf{x}^{T} & 1
\end{array}\right] & b-\mathbf{a}^{T} \mathbf{x}-2 s
\end{array}\right] \succeq 0 \text {. }
\end{aligned}
$$

Here, we see that an uncertain semi-infinite linear inequality becomes a LMI.

\subsection{Robust Stochastic Optimisation}

Robust stochastic optimisation $[28,193]$ methods are used when the knowledge of the underlying stochastic model for the uncertain data exists or can be approximated. The objectives and constraints are defined by averaging possible outcomes or by considering probabilities of events of interest [193]. A robust stochastic optimisation problem where the objective and constraints are defined by the expected 
value of some function of the uncertain data is stated below.

$$
\begin{array}{cl}
\min _{\mathbf{x}} & \mathbb{E}\left\{f_{0}\left(\mathbf{x}, \mathbf{u}_{0}\right)\right\} \\
\text { s.t. } & \mathbb{E}\left\{f_{i}\left(\mathbf{x}, \mathbf{u}_{i}\right)\right\} \leq 0, \quad i=1 \ldots l
\end{array}
$$

where $\mathbf{x} \in \mathbb{R}^{n}$ is the optimisation variable, $f_{0}, f_{i}: \mathbb{R}^{n} \rightarrow \mathbb{R}$ are functions and $\mathbf{u}_{i} \in \mathbb{R}^{k}$ is the uncertain data drawn from the sample space $\mathcal{S}_{i}$. It is assumed that the probability distribution function of $\mathcal{S}_{i}$ is known. When considering probabilities of events, the robust stochastic optimisation problem is given by

$$
\begin{array}{cl}
\min _{\mathbf{x}} & \operatorname{Pr}\left\{f_{0}\left(\mathbf{x}, \mathbf{u}_{0}\right)\right\} \\
\text { s.t. } & \operatorname{Pr}\left\{f_{i}\left(\mathbf{x}, \mathbf{u}_{i}\right)\right\} \leq \alpha_{i}, \quad i=1 \ldots l
\end{array}
$$

where $\alpha_{i}, i=1 \ldots l$ are the limits on the probabilities. Any combination of expectation and probabilities of functions can be used in a robust stochastic optimisation problem.

\section{Stochastic Linear Optimisation}

Consider the following robust stochastic LP where the data vector $\mathbf{a}$ is a Gaussian random vector with mean $\overline{\mathbf{a}}$ and covariance $\boldsymbol{\Sigma}$.

$$
\begin{array}{cl}
\min _{\mathbf{x}} & \mathbf{c}^{T} \mathbf{x} \\
\text { s.t. } & \operatorname{Pr}\left\{\mathbf{a}^{T} \mathbf{x} \leq b\right\} \geq \alpha .
\end{array}
$$

It is required that the constraint holds with a probability greater than $\alpha$. The mean, $\mu$, and the variance, $\sigma^{2}$, of the random variable $\mathbf{a}^{T} \mathbf{x}$ are given by $\overline{\mathbf{a}}^{T} \mathbf{x}$ and $\mathbf{x}^{T} \mathbf{\Sigma} \mathbf{x}$, respectively. Since $\mathbf{a}^{T} \mathbf{x}$ is a Gaussian random variable, its cumulative distribution function is given by [101]

$$
\mathrm{F}(u)=\frac{1}{2}\left[1+\operatorname{erf}\left(\frac{u-\mu}{\sqrt{2 \sigma^{2}}}\right)\right],
$$

where erf is the error function defined as [101]

$$
\operatorname{erf}(z)=\frac{2}{\sqrt{\pi}} \int_{0}^{z} \exp \left(-t^{2}\right) \mathrm{d} t
$$

Utilising (3.17), $\operatorname{Pr}\left\{\mathbf{a}^{T} \mathbf{x} \leq b\right\}$ is expressed as

$$
\operatorname{Pr}\left\{\mathbf{a}^{T} \mathbf{x} \leq b\right\}=\frac{1}{2}\left[1+\operatorname{erf}\left(\frac{b-\mathbf{a}^{T} \mathbf{x}}{\sqrt{2 \mathbf{x}^{T} \mathbf{\Sigma} \mathbf{x}}}\right)\right],
$$


and the constraint can be restated as

$$
\sqrt{2} \operatorname{erf}^{-1}(2 \alpha-1)\left\|\Sigma^{\frac{1}{2}} \mathbf{x}\right\|_{2}+\mathbf{a}^{T} \mathbf{x} \leq b
$$

When $\alpha \geq \frac{1}{2}, \operatorname{erf}^{-1}(2 \alpha-1) \geq 0$, hence (3.20) becomes a second-order cone constraint and (3.16) can be restated as the following SOCP

$$
\begin{array}{ll}
\min _{\mathbf{x}} & \mathbf{c}^{T} \mathbf{x} \\
\text { s.t. } & \sqrt{2} \operatorname{erf}^{-1}(2 \alpha-1)\left\|\boldsymbol{\Sigma}^{\frac{1}{2}} \mathbf{x}\right\|_{2}+\mathbf{a}^{T} \mathbf{x} \leq b .
\end{array}
$$

\section{Stochastic Least Squares Optimisation}

Consider the following unconstrained least squares problem.

$$
\min _{\mathbf{x}} \quad\|\mathbf{A} \mathbf{x}-\mathbf{b}\|_{2}^{2}
$$

where $\mathbf{A} \in \mathbb{R}^{m \times n}$ is a random matrix given by $\mathbf{A}=\overline{\mathbf{A}}+\mathbf{E}$. Here, $\overline{\mathbf{A}}$ is the mean of $\mathbf{A}$ and $\mathbf{E}$ is a zero mean random matrix. Problem (3.22) can be transformed into a robust stochastic optimisation problem by restating the problem in terms of the expected value of the objective, i.e.,

$$
\min _{\mathbf{x}} \mathbb{E}\left\{\|\mathbf{A x}-\mathbf{b}\|_{2}^{2}\right\}
$$

The objective can be expressed as

$$
\begin{aligned}
\mathbb{E}\left\{\|\mathbf{A} \mathbf{x}-\mathbf{b}\|_{2}^{2}\right\} & =\mathbb{E}\left\{((\overline{\mathbf{A}}+\mathbf{E}) \mathbf{x}-\mathbf{b})^{T}((\overline{\mathbf{A}}+\mathbf{E}) \mathbf{x}-\mathbf{b})\right\} \\
& =\|\overline{\mathbf{A}} \mathbf{x}-\mathbf{b}\|_{2}^{2}+\mathbf{x}^{T} \mathbf{\Sigma} \mathbf{x}
\end{aligned}
$$

where $\boldsymbol{\Sigma}=\mathbb{E}\left\{\mathbf{E}^{T} \mathbf{E}\right\}$ is the covariance of $\mathbf{E}$. The robust stochastic least squares problem is therefore given by

$$
\min _{\mathbf{x}} \quad\|\overline{\mathbf{A}} \mathbf{x}-\mathbf{b}\|_{2}^{2}+\left\|\boldsymbol{\Sigma}^{\frac{1}{2}} \mathbf{x}\right\|_{2}^{2}
$$

By utilising the KKT conditions, specifically that the gradient of the objective vanishes at the optimum, it can be shown that the above problem has an analytical solution given by

$$
\mathbf{x}^{*}=\left(\overline{\mathbf{A}}^{T} \overline{\mathbf{A}}+\boldsymbol{\Sigma}\right)^{-1} \overline{\mathbf{A}}^{T} \mathbf{b}
$$




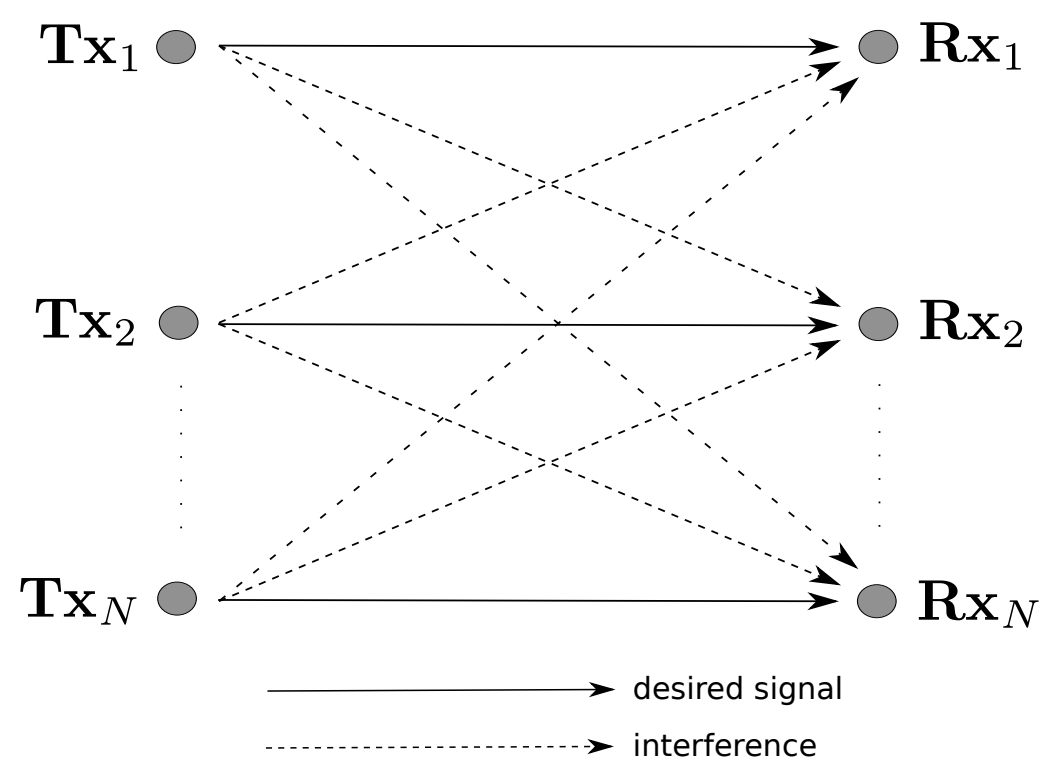

Figure 3.2: Multi-user wireless network.

\subsection{Application of Robust Optimisation in Com- munication Systems}

In this section we present a number of applications of robust optimisation in communication systems. We will mainly focus on robust power control and robust conventional and cooperative beamforming.

\subsubsection{Power Control in Wireless Networks}

Transmit power control provides a method for controlling interference and for increased utilisation of the wireless spectrum in multi-user communication systems where the users access the shared channel simultaneously. Power control in wireless networks has been extensively studied based on different transmission models and application needs $[3,5,14,46,73,75,117,133,139,165,176,186,196,223,253-$ 255, 259]. In [117] and later in [46], geometric programming was used to formulate and solve power control problems. The main advantage of using geometric programming is that globally optimal power allocations can be efficiently found.

Fig. 3.2 shows the wireless network considered in $[46,117]$. The network consists of $N$ transmitter and receiver pairs. Receiver $i$ is meant to receive the signal from transmitter $i$. The transmitters transmit at power level $p_{1}, \ldots, p_{N}$, which are the optimisation variables in the power control optimisation problems. Independent, point-to-point, flat Rayleigh fading channels are assumed for all links in the network. The instantaneous power of the channel between the $j$ th transmitter and the $i$ th receiver is represented by $g^{(i j)}$ and has mean $\mathbb{E}\left\{g^{(i j)}\right\}=G^{(i j)}$. 
The SINR at the $i$ th receiver is given by

$$
\gamma^{(i)}=\frac{p_{i} g^{(i i)}}{\sum_{j=1, j \neq i}^{N} p_{j} g^{(i j)}+\sigma_{i}^{2}},
$$

where $\sigma_{i}^{2}$ is the noise power at receiver $i$. Since the channel power is a random variable, $\gamma^{(i)}$ is also a random variable.

In [117], a stochastic optimisation approach is taken and the robust power control problems are formulated based on the outage probability of the receivers. The $i$ th receiver outage probability is defined as the probability that the SINR at the $i$ th receiver is below some predefined threshold $\gamma^{T}$, i.e.,

$$
\begin{aligned}
\mathrm{P}_{\mathrm{o}}{ }^{(i)} & =\operatorname{Pr}\left(\gamma^{(i)} \leq \gamma^{T}\right) \\
& =\operatorname{Pr}\left(p_{i} g^{(i i)} \leq \gamma^{T}\left(\sum_{j=1, j \neq i}^{N} p_{j} g^{(i j)}+\sigma_{i}^{2}\right)\right) .
\end{aligned}
$$

To simplify the analysis of the outage probability and the resulting optimisation problems, noise power is assumed to be insignificant compared to the interference powers and hence receiver noise is disregarded in [117]. Therefore, the outage probability can be restated as

$$
\mathrm{P}_{\mathrm{o}}{ }^{(i)}=\operatorname{Pr}\left(p_{i} g^{(i i)}-\gamma^{T} \sum_{j=1, j \neq i}^{N} p_{j} g^{(i j)} \leq 0\right) .
$$

Since the channel powers are independent exponentially distributed random variables, the outage probability density function (PDF) is that of a difference between an exponential random variable and the sum of $N-1$ exponentially distributed random variables, and therefore the outage probability is known to have the following form $[222,251,252]$

$$
\mathrm{P}_{\mathrm{o}}{ }^{(i)}=1-\prod_{j \neq i}^{N}\left(\frac{1}{1+\frac{\gamma^{T} G^{(i j)} p_{j}}{G^{(i i)} p_{i}}}\right) .
$$

The first robust power control problem considered in [117] is the minimisation of the maximum outage probability subject to box (minimum and maximum) constraints on the transmit powers. This problem is mathematically represented as

$$
\begin{aligned}
\min _{\left\{p_{i}\right\}_{i=1}^{N}} & \max _{i}\left(1-\prod_{j \neq i}^{N}\left(\frac{1}{1+\frac{\gamma^{T} G^{(i j)} p_{j}}{G^{(i i)} p_{i}}}\right)\right) \\
\text { s.t. } & p_{\min } \leq p_{i} \leq p_{\max }, \quad i=1,2, \ldots, N
\end{aligned}
$$


where $p_{\min }$ and $p_{\max }$ are the minimum and maximum limits on transmit powers, respectively. We note that problem (3.31) can be written in the following equivalent form

$$
\begin{aligned}
\min _{\left\{p_{i}\right\}_{i=1}^{N}} & \max _{i}\left(\prod_{j \neq i}^{N}\left(1+\frac{\gamma^{T} G^{(i j)} p_{j}}{G^{(i i)} p_{i}}\right)\right) \\
\text { s.t. } & p_{\min } \leq p_{i} \leq p_{\max }, \quad i=1,2, \ldots, N
\end{aligned}
$$

By introducing the auxiliary variable $t$, problem (3.31) can be rewritten in the epigraph form as

$$
\begin{aligned}
& \min _{t,\left\{p_{i}\right\}_{i=1}^{N}} t \\
& \text { s.t. } \quad \prod_{j \neq i}^{N}\left(1+\frac{\gamma^{T} G^{(i j)} p_{j}}{G^{(i i)} p_{i}}\right) \leq t, \quad i=1,2, \ldots, N \\
& p_{\min } \leq p_{i} \leq p_{\max }, \quad i=1,2, \ldots, N
\end{aligned}
$$

Problem (3.33) is recognised as a GP, the objective is a monomial and the first and second constraints are a posynomial and monomial, respectively, and hence it can be transformed into a convex optimisation problem and solved efficiently.

The second robust power control considered in [117] is the maximisation of the minimum expected value of the receiver signal to interference ratio (SIR) subject to non-negativity constraints on the transmit powers. The expected value of the $i$ th SIR is given by

$$
\begin{aligned}
\operatorname{SIR}_{\mathrm{E}}^{(i)} & =\mathbb{E}\left\{\frac{p_{i} g^{(i i)}}{\sum_{j=1, j \neq i}^{N} p_{j} g^{(i j)}}\right\} \\
& =\frac{p_{i} G^{(i i)}}{\sum_{j=1, j \neq i}^{N} p_{j} G^{(i j)}}
\end{aligned}
$$

The optimisation problem is expressed as

$$
\begin{aligned}
\max _{\left\{p_{i}\right\}_{i=1}^{N}} & \min _{i}\left(\frac{p_{i} G^{(i i)}}{\sum_{j=1, j \neq i}^{N} p_{j} G^{(i j)}}\right) \\
\text { s.t. } & p_{i}>0, \quad i=1,2, \ldots, N
\end{aligned}
$$

In [117], it was shown that at the optimum the SIR at all receivers will be equal. Using this observation, problem (3.35) can be restated in the epigraph form [29] as

$$
\begin{array}{rl}
\max _{t,\left\{p_{i}\right\}_{i=1}^{N}} & t \\
\text { s.t. } & \left(\frac{p_{i} G^{(i i)}}{\sum_{j=1, j \neq i}^{N} p_{j} G^{(i j)}}\right)=t, \quad i=1,2, \ldots, N
\end{array}
$$




$$
p_{i}>0, \quad i=1,2, \ldots, N
$$

The solution to problem (3.36) is given by the Perron-Frobenius theorem for the maximum eigenvalue of a matrix that has non-negative elements [165]. By defining $\tau=1 / t$, problem (3.36) can be restated as

$$
\begin{aligned}
\min _{\tau,\left\{p_{i}\right\}_{i=1}^{N}} & \tau \\
\text { s.t. } & \mathbf{A p}=\tau \mathbf{p} \\
& p_{i}>0, \quad i=1,2, \ldots, N
\end{aligned}
$$

where $\mathbf{p} \triangleq\left[p_{1}, p_{2}, \ldots, p_{N}\right]$ and the matrix $\mathbf{A}$ is defined as

$$
\mathbf{A}_{i j}=\frac{G^{(i j)}}{G^{(i i)}}, i \neq j \quad \mathbf{A}_{i i}=0
$$

Problem (3.37) is recognised as a problem for finding the largest eigenvalue of the matrix A [29]. According to Perron-Frobenius theory, the eigenvalue $\lambda$ of $\mathbf{A}$ that is largest in magnitude is real and positive and has an associated eigenvector $\mathbf{v}$ with all positive entries [117]. The eigenvector $\mathbf{v}$ and the eigenvalue $\lambda$ are called the PerronFrobenius eigenvector and eigenvalue of $\mathbf{A}$. The Perron-Frobenius eigenvector $\mathbf{v}$ gives the optimal power allocation, i.e., $p_{i}=\mathbf{v}_{i}[117]$.

The third problem analysed in [117] is the minimisation of total transmit power subject to outage probability constraints and bounds on individual transmit powers. This problem is expressed as

$$
\begin{aligned}
& \min _{\left\{p_{i}\right\}_{i=1}^{N}} \sum_{i=1}^{N} p_{i} \\
& \text { s.t. } \quad 1-\prod_{j \neq i}^{N}\left(\frac{1}{1+\frac{\gamma^{T} G^{(i j)} p_{j}}{G^{(i i)} p_{i}}}\right) \leq \mathrm{P}_{\mathrm{o}, \max }^{(i)} \\
& p_{\text {min }} \leq p_{i} \leq p_{\text {max }}, \quad i=1,2, \ldots, N
\end{aligned}
$$

After straightforward manipulation of the outage probability constraint, problem (3.38) can be restated as

$$
\begin{aligned}
\min _{\left\{p_{i}\right\}_{i=1}^{N}} & \sum_{i=1}^{N} p_{i} \\
\text { s.t. } & \left(1-\mathrm{P}_{\mathrm{o}, \max }^{(i)}\right) \prod_{j \neq i}^{N}\left(1+\frac{\gamma^{T} G^{(i j)} p_{j}}{G^{(i i)} p_{i}}\right) \leq 1 \\
& p_{\min } \leq p_{i} \leq p_{\max }, \quad i=1,2, \ldots, N
\end{aligned}
$$

Problem (3.39) is recognised as a GP, the objective is a posynomial and the first 


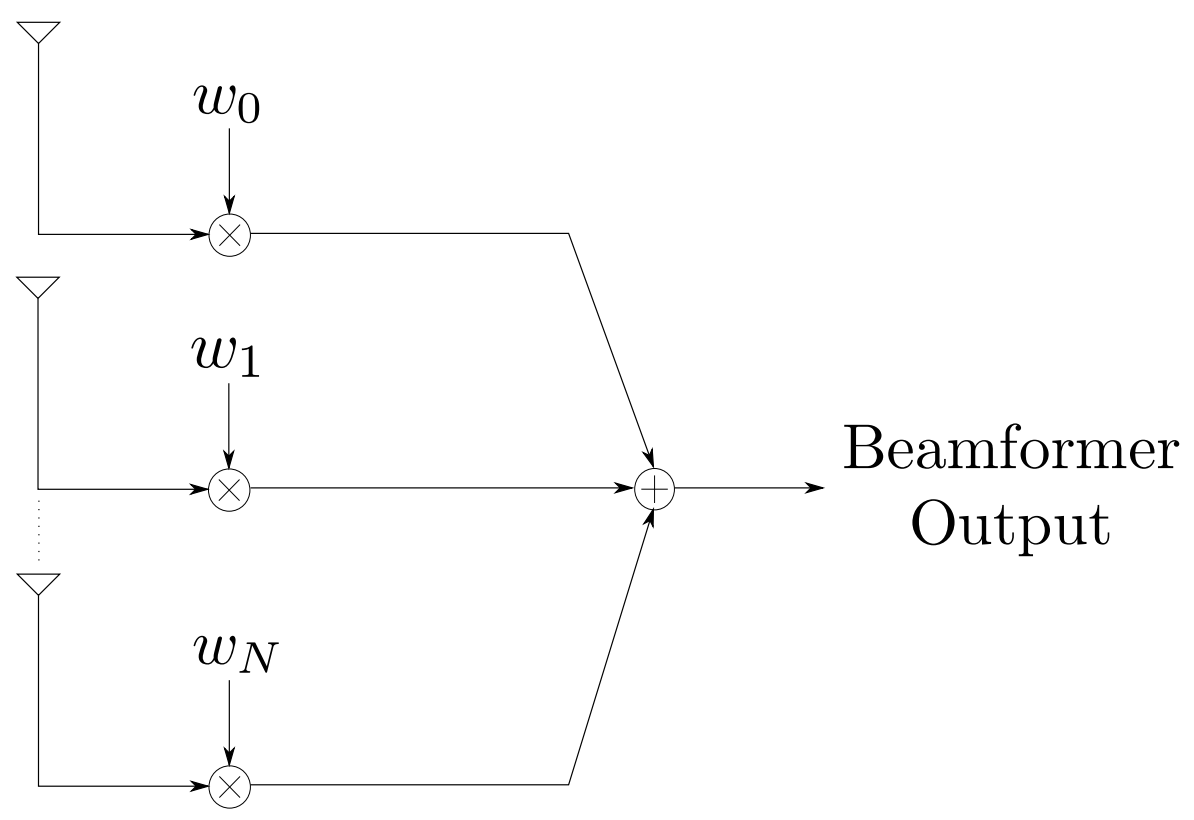

Figure 3.3: Receive beamformer.

and second constraints are posynomial and monomial, respectively, and hence it can be transformed into a convex optimisation problem and solved efficiently.

\subsubsection{Receive Beamforming}

Receive beamforming is widely used to improve system performance in wireless communications $[88,182]$, radar $[31,104]$, sonar $[55,81,131]$, audio processing [116, 279], radio astronomy [169], biomedicine [200], and many other signal processing systems. Fig. 3.3 shows an example of a receive beamformer consisting of an $N$ antenna array.

The output of the narrowband beamformer is given by

$$
y(k)=\mathbf{w}^{H} \mathbf{x}(k),
$$

where $k$ is the time index, $\mathbf{x}(k)=\left[x_{1}(k), \ldots, x_{N}(k)\right]^{T} \in \mathbb{C}^{N}$ is the complex vector of the sampled array output and $\mathbf{w}=\left[w_{1}, \ldots, w_{N}\right]^{T} \in \mathbb{C}^{N}$ is the complex vector of beamformer weights. The sampled array output is given by

$$
\mathbf{x}(k)=s(k) \mathbf{a}(\theta)+\mathbf{v}(k),
$$

where $s(k)$ is the desired narrowband signal impinging upon the array from angle $\theta$ from far-field, $\mathbf{a}(\theta) \in \mathbb{C}^{N}$ is the response of the array to a plane wave arriving from angle $\theta$; commonly referred to as the steering vector or the array manifold, and $\mathbf{v}(k)$ is the interference-plus-noise term. The SINR, $\gamma$, at the beamformer output 
is expressed as

$$
\gamma=\frac{\sigma_{s}^{2}\left|\mathbf{w}^{H} \mathbf{a}(\theta)\right|^{2}}{\mathbf{w}^{H} \mathbf{R}_{v} \mathbf{w}}
$$

where $\sigma_{s}^{2}$ is the desired signal power and $\mathbf{R}_{v}$ is the interference-plus-noise covariance matrix. The aim is to design the optimum beamformer weight vector that maximises $\gamma$. This can be achieved by finding the weight vector that makes $\mathbf{w}^{H} \mathbf{a}(\theta) \approx 1$, i.e., maintaining a distortionless response to the desired signal, and $\mathbf{w}^{H} \mathbf{v}$ small $[142,143,168]$. If $\mathbf{a}(\theta)$ and $\mathbf{R}_{v}$ are known, then this equates to solving the following optimisation problem

$$
\begin{array}{ll}
\min _{\mathbf{w}} & \mathbf{w}^{H} \mathbf{R}_{v} \mathbf{w} \\
\text { s.t. } & \mathbf{w}^{H} \mathbf{a}(\theta)=1 .
\end{array}
$$

An analytical solution to the above problem can be obtained by forming the two KKT conditions that the gradient of the Lagrangian vanishes at the optimum and the problem is primal feasible, i.e.,

$$
\begin{aligned}
2 \mathbf{R}_{v} \mathbf{w}^{*}+\lambda^{*} \mathbf{a}(\theta) & =0, \\
\mathbf{w}^{* H} \mathbf{a}(\theta) & =1,
\end{aligned}
$$

where $\mathbf{w}^{*}$ and $\lambda^{*}$ are the primal and dual optimum values. Solving this set of equations gives the the optimum beamformer weights which are expressed as

$$
\mathbf{w}^{*}=\frac{\mathbf{R}_{v}^{-1} \mathbf{a}(\theta)}{\mathbf{a}^{H}(\theta) \mathbf{R}_{v}^{-1} \mathbf{a}(\theta)}
$$

The above solution is known as the minimum variance distortionless response (MVDR) beamformer [142, 143, 168, 280].

In practise, $\mathbf{R}_{v}$ is seldom exactly known and it needs to be estimated from the received samples $\mathbf{x}$. This estimate is known as the sample covariance matrix and is given by

$$
\hat{\mathbf{R}}_{v}=\frac{1}{L} \sum_{i=1}^{L} \mathbf{x}(i) \mathbf{x}^{H}(i),
$$

where $L$ is the number of samples used for the estimation. Using (3.45), one obtains the following solution.

$$
\mathbf{w}^{*}=\frac{\hat{\mathbf{R}}_{v}^{-1} \mathbf{a}(\theta)}{\mathbf{a}^{H}(\theta) \hat{\mathbf{R}}_{v}^{-1} \mathbf{a}(\theta)}
$$

This solution is known as the sample matrix inversion (SMI) algorithm [187] (also 
commonly referred to as Capon's method [35]).

Early methods $[35,93,168,187]$ were developed based on the assumption that the desired signal components were not present in the training data used for the estimation (3.45). While this assumption is valid for some applications, it does not hold for many applications such as wireless communications. In [71], it was shown that the performance of the SMI algorithm and other adaptive beamforming techniques are significantly degraded when the desired signal components are present in the training data. Uncertainty in the knowledge of the steering vector is also known to cause substantial performance losses in adaptive beamforming techniques, especially when the desired signal components are present in the training data $[7,27,83,87,106,113,127,190]$. The uncertainty in the steering vector arises principally from three sources [143]:

- uncertainty in the angle of arrival,

- uncertainty in the array manifold given perfect knowledge of the angle of arrival, and

- variations in the gains of the signal-processing paths.

Several robust adaptive beamforming techniques have been proposed to overcome the problem of uncertainty in the steering vector. These include introducing point mainbeam constraints [131], diagonal loading [36, 56, 70], eigenvalue thresholding [98] and eigenspace-based beamforming [40,71]. These methods suffer from the drawback that one needs to design a parameter which is not easy to compute from the level of uncertainty. For instance, the diagonal loading method requires one to find the diagonal loading factor but it is not clear how to obtain the optimal value of this based on the known level of uncertainty [238]. To overcome this shortcoming, designs based on ellipsoidal [137, 142,143,221] and Euclidean ball $[237,238]$ uncertainty sets have been proposed in the literature.

The ellipsoidal uncertainty set model can be used to design a robust beamformer as follows. The robust beamforming problem can be restated as $[142,143]$

$$
\begin{array}{ll}
\min _{\mathbf{w}} & \mathbf{w}^{H} \mathbf{R}_{v} \mathbf{w} \\
\text { s.t. } & \Re\left\{\mathbf{w}^{H} \mathbf{a}\right\} \geq 1, \quad \forall \mathbf{a} \in \mathcal{U},
\end{array}
$$

where $\mathcal{U}$ is an ellipsoidal uncertainty set that covers all possible realisations of $\mathbf{a}(\theta)$. The constraint $\Re\left\{\mathbf{w}^{H} \mathbf{a}\right\} \geq 1$ is used for two reasons. First, as will be shown later, this allows the semi-infinite constraint to be expressed as a SOC constraint. Second, the real part of $\mathbf{w}^{H} \mathbf{a}$ is an efficient lower bound for its magnitude, since the objective is unchanged if the weight vector undergoes an arbitrary phase shift $[142,143]$. The authors of $[142,143]$ argue that it is unnecessary to constrain the imaginary part of 
$\mathbf{w}^{H} \mathbf{a}$ to zero, since the same rotation that maximises the real part for a given level of $\mathbf{w}^{H} \mathbf{R}_{v} \mathbf{w}$ simultaneously minimises the imaginary component of the response.

Using the following definitions

$$
\mathbf{x}=\left[\begin{array}{c}
\Re\{\mathbf{w}\} \\
\Im\{\mathbf{w}\}
\end{array}\right] \quad \mathbf{z}=\left[\begin{array}{c}
\Re\{\mathbf{a}\} \\
\Im\{\mathbf{a}\}
\end{array}\right] \quad \mathbf{R}=\left[\begin{array}{cc}
\Re\left\{\mathbf{R}_{v}\right\} & -\Im\left\{\mathbf{R}_{v}\right\} \\
\Im\left\{\mathbf{R}_{v}\right\} & \Re\left\{\mathbf{R}_{v}\right\}
\end{array}\right]
$$

problem (3.47) can be transformed into the following real valued problem $[142,143]$

$$
\begin{array}{ll}
\min _{\mathbf{x}} & \mathbf{x}^{T} \mathbf{R} \mathbf{x} \\
\text { s.t. } & \mathbf{x}^{T} \mathbf{z} \geq 1, \quad \forall \mathbf{z} \in \mathcal{U},
\end{array}
$$

where $\mathcal{U}=\left\{\mathbf{z}=\tilde{\mathbf{z}}+\mathbf{A u} \mid\|\mathbf{u}\|_{2} \leq 1\right\}$. Using (3.5) and (3.6), the constraint of the above problem can be restated as the following SOC constraint

$$
\left\|\mathbf{A}^{T} \mathbf{x}\right\|_{2} \leq \tilde{\mathbf{z}}^{T} \mathbf{x}-1
$$

and the robust MVDR beamforming problem as the following SOCP

$$
\begin{array}{cl}
\min _{\mathbf{x}} & \mathbf{x}^{T} \mathbf{R} \mathbf{x} \\
\text { s.t. } & \left\|\mathbf{A}^{T} \mathbf{x}\right\|_{2} \leq \tilde{\mathbf{z}}^{T} \mathbf{x}-1 .
\end{array}
$$

The uncertainty ellipsoid can be computed in a number of ways. If the array manifold can be measured in a controlled manner, the ellipsoid describing it could be generated from the mean and covariance of the measurements [143]. When measurements are not performed, the ellipsoid could be predicted from numerical simulations that take into account variation in the array response due to manufacturing tolerance, termination impedance, and similar effects [143].

In $[237,238]$, a robust MVDR beamformer is designed using the Euclidean ball uncertainty set model for the error in the steering vector. The steering vector is modelled as

$$
\mathbf{a}=\tilde{\mathbf{a}}+\mathbf{e}
$$

where $\mathbf{a}$ and $\tilde{\mathbf{a}}$ are the actual and presumed steering vectors, respectively, and e is a complex vector that describes the effect of the steering vector distortions. Additionally, the norm of $\mathbf{e}$ is constrained to be less than some $\epsilon>0$, i.e., $\|\mathbf{e}\|_{2} \leq \epsilon$. Hence, the steering vector comes from the set

$$
\mathcal{U}=\left\{\mathbf{a} \mid \mathbf{a}=\tilde{\mathbf{a}}+\mathbf{e},\|\mathbf{e}\|_{2} \leq \epsilon\right\}
$$

The constraint that the absolute value of the array response should not be 
smaller than one is imposed in the robust beamformer design problem, i.e.,

$$
\left|\mathbf{w}^{H} \mathbf{a}\right| \geq 1, \quad \forall \mathbf{a} \in \mathcal{U}
$$

This constraint guarantees that a distortionless response is maintained in the worstcase scenario, i.e., for the smallest value of $\left|\mathbf{w}^{H} \mathbf{a}\right|$. The robust beamforming problem can therefore be expressed as

$$
\begin{array}{ll}
\min _{\mathbf{w}} & \mathbf{w}^{H} \mathbf{R}_{v} \mathbf{w} \\
\text { s.t. } & \left|\mathbf{w}^{H} \mathbf{a}\right| \geq 1, \quad \forall \mathbf{a} \in \mathcal{U}
\end{array}
$$

Problem (3.55) is a non-convex semi-infinite problem. It is non-convex because the absolute value operator in the constraint is a non-convex operator [29] and semi-infinite due to the infinite number of constraints arising from the uncertainty in the steering vector. The infinite number of constraints can be transformed into a single constraint by only considering the worst-case scenario, i.e., maintaining a distortionless response for the smallest value of $\left|\mathbf{w}^{H} \mathbf{a}\right|$. Mathematically, this is represented as

$$
\min _{\mathbf{a} \in \mathcal{U}}\left|\mathbf{w}^{H} \mathbf{a}\right| \geq 1
$$

Using (3.52) and (3.53), the above constraint can be rewritten as

$$
\min _{\mathbf{e} \in \mathcal{V}}\left|\mathbf{w}^{H} \tilde{\mathbf{a}}+\mathbf{w}^{H} \mathbf{e}\right| \geq 1
$$

where $\mathcal{V}=\left\{\mathbf{e} \mid\|\mathbf{e}\|_{2} \leq \epsilon\right\}$. Applying the triangle and Cauchy-Schwarz inequalities and the inequality $\|\mathbf{e}\|_{2} \leq \epsilon$, we have that [238]

$$
\left|\mathbf{w}^{H} \tilde{\mathbf{a}}+\mathbf{w}^{H} \mathbf{e}\right| \geq\left|\mathbf{w}^{H} \tilde{\mathbf{a}}\right|-\left|\mathbf{w}^{H} \mathbf{e}\right| \geq\left|\mathbf{w}^{H} \tilde{\mathbf{a}}\right|-\epsilon\|\mathbf{w}\|_{2} .
$$

Hence, the right hand side of (3.58) provides a lower bound for $\left|\mathbf{w}^{H} \tilde{\mathbf{a}}+\mathbf{w}^{H} \mathbf{e}\right|$.

Then, using (3.58), the robust beamforming problem becomes

$$
\begin{array}{ll}
\min _{\mathbf{w}} & \mathbf{w}^{H} \mathbf{R}_{v} \mathbf{w} \\
\text { s.t. } & \left|\mathbf{w}^{H} \tilde{\mathbf{a}}\right|-\epsilon\|\mathbf{w}\|_{2} \geq 1 .
\end{array}
$$

Problem (3.59) is still non-convex due to the absolute value operator in the constraint. However, again noting that an arbitrary phase shift applied to the beamforming vector does not change the objective, w can be chosen such that $\Re\left\{\mathbf{w}^{H} \tilde{\mathbf{a}}\right\} \geq$ 0 and $\Im\left\{\mathbf{w}^{H} \tilde{\mathbf{a}}\right\}=0$. The problem can therefore be transformed into a convex prob- 
lem with a quadratic objective with SOC and linear constraints, i.e.,

$$
\begin{array}{cl}
\min _{\mathbf{w}} & \mathbf{w}^{H} \mathbf{R}_{v} \mathbf{w} \\
\text { s.t. } & \mathbf{w}^{H} \tilde{\mathbf{a}} \geq \epsilon\|\mathbf{w}\|_{2}+1, \\
& \Im\left\{\mathbf{w}^{H} \tilde{\mathbf{a}}\right\}=0 .
\end{array}
$$

Note that the two constraints in the above problem ensure that $\Re\left\{\mathbf{w}^{H} \tilde{\mathbf{a}}\right\} \geq 0$, thus it is not required to add this constraint to the problem [238].

In [236], a stochastic approach is taken to design the robust MVDR beamformer. Here, the steering vector error, e, in (3.52) is considered to be a random variable with a known probability distribution function. Although the worst-case based beamformers are known to be quite robust, they tend to be too conservative. This is because the design protects against the worst-case scenario, which in practice may occur with a very low probability. In [236], the deterministic distortionless response constraint is transformed into a stochastic constraint which allows the constraint to be satisfied with a certain prescribed probability. An important advantage of this approach over the worst-case approach is that this approach enables better specification of the parameters of the uncertainty region by explicity quantifying these parameters in terms of the beamformer outage probability and second-order statistics of the steering vector errors [236].

The probability-constrained beamformer can therefore be expressed as [236]

$$
\begin{array}{ll}
\min _{\mathbf{w}} & \mathbf{w}^{H} \mathbf{R}_{v} \mathbf{w} \\
\text { s.t. } & \operatorname{Pr}\left\{\left|\mathbf{w}^{H} \mathbf{a}\right| \geq 1\right\} \geq \alpha,
\end{array}
$$

where $\alpha$ is a certain prescribed probability. Note that $1-\alpha$ is the beamformer outage probability, i.e., the probability of the distortionless response constraint not being satisfied. The constraint in its current form is quite difficult to deal with. However, when the steering vector errors are reasonably small, i.e., $\left|\mathbf{w}^{H} \tilde{\mathbf{a}}\right|>\left|\mathbf{w}^{H} \mathbf{e}\right|$, then by applying the triangle inequality, one obtains the following simplified form [236]

$$
\left|\mathbf{w}^{H}(\tilde{\mathbf{a}}+\mathbf{e})\right| \geq\left|\mathbf{w}^{H} \tilde{\mathbf{a}}\right|-\left|\mathbf{w}^{H} \mathbf{e}\right|
$$

Using this lower bound, the probability based constraint can be approximated as

$$
\operatorname{Pr}\left\{\left|\mathbf{w}^{H} \mathbf{e}\right| \leq\left|\mathbf{w}^{H} \tilde{\mathbf{a}}\right|-1\right\} \geq \alpha \text {. }
$$

Using (3.63), the simplified probability-constrained beamformer can be restated as

$$
\min _{\mathbf{w}} \quad \mathbf{w}^{H} \mathbf{R}_{v} \mathbf{w}
$$




$$
\text { s.t. } \quad \operatorname{Pr}\left\{\left|\mathbf{w}^{H} \mathbf{e}\right| \leq\left|\mathbf{w}^{H} \tilde{\mathbf{a}}\right|-1\right\} \geq \alpha \text {. }
$$

If $\mathbf{e}$ is drawn from a complex circularly symmetric Gaussian distribution with zero mean and the covariance matrix $\boldsymbol{\Sigma}_{\mathbf{e}}$, i.e., $\mathbf{e} \sim \mathcal{N}_{\mathcal{C}}\left(\mathbf{0}, \boldsymbol{\Sigma}_{\mathbf{e}}\right)$, then $\mathbf{w}^{H} \mathbf{e}$ is a complex Gaussian random variable with the distribution $\mathbf{w}^{H} \mathbf{e} \sim \mathcal{N}_{\mathcal{C}}\left(\mathbf{0},\left\|\Sigma_{\mathbf{e}}^{\frac{1}{2}} \mathbf{w}\right\|_{2}^{2}\right)$. As a result, $\left|\mathbf{w}^{H} \mathbf{e}\right|$ is Rayleigh-distributed with the CDF given by [179]

$$
\mathrm{F}(u)=1-\exp \left(-\frac{u^{2}}{\left\|\Sigma_{\mathbf{e}}^{\frac{1}{2}} \mathbf{w}\right\|_{2}^{2}}\right) .
$$

Using (3.65), the probability based distortionless response constraint can be stated as

$$
\left\|\boldsymbol{\Sigma}_{\mathbf{e}}^{\frac{1}{2}} \mathbf{w}\right\|_{2} \leq \frac{1}{-\log (1-\alpha)}\left(\left|\mathbf{w}^{H} \tilde{\mathbf{a}}\right|-1\right)
$$

Due to the absolute value operator, this is a non-convex constraint. Observing that the objective in problem (3.64) is unchanged when $\mathbf{w}$ undergoes an arbitrary phase rotation, $\mathbf{w}^{H} \tilde{\mathbf{a}}$ can be chosen to be real without loss of generality [236]. The probability-constrained robust beamformer design problem can then be written as the following convex SOCP problem

$$
\begin{array}{ll}
\min _{\mathbf{w}} & \mathbf{w}^{H} \mathbf{R}_{v} \mathbf{w} \\
\text { s.t. } & \left\|\boldsymbol{\Sigma}_{\mathbf{e}}^{\frac{1}{2}} \mathbf{w}\right\|_{2} \leq \frac{1}{-\log (1-\alpha)}\left(\mathbf{w}^{H} \tilde{\mathbf{a}}-1\right), \\
& \Re\left\{\mathbf{w}^{H} \tilde{\mathbf{a}}\right\} \geq 0, \\
& \Im\left\{\mathbf{w}^{H} \tilde{\mathbf{a}}\right\}=0 .
\end{array}
$$

\subsubsection{Transmit Beamforming}

Transmit beamforming can be used in either single or multi-user (MU) systems. In a MU system, transmit beamforming can be used to deliver independent information streams to each user or common information to all users. The former is known as unicast beamforming [24, 25, 82, 183, 235], while the latter is referred to as multicast or broadcast [41, 82, 119, 120,211,212] beamforming.

Transmit beamforming, especially in a MU system, is generally more difficult than receive beamforming, because a receive beamformer only affects the signal quality of one user, whereas, the signal transmitted from a transmit beamformer is not only received by the intended user but all other users in the network. Hence, the design of transmit beamformers involves the consideration of the performance of all users in the network. Another difference between receive and transmit beamforming is the channel knowledge. Channel state information at the transmitter (CSIT) is generally not required in a system employing receive beamforming while it is 


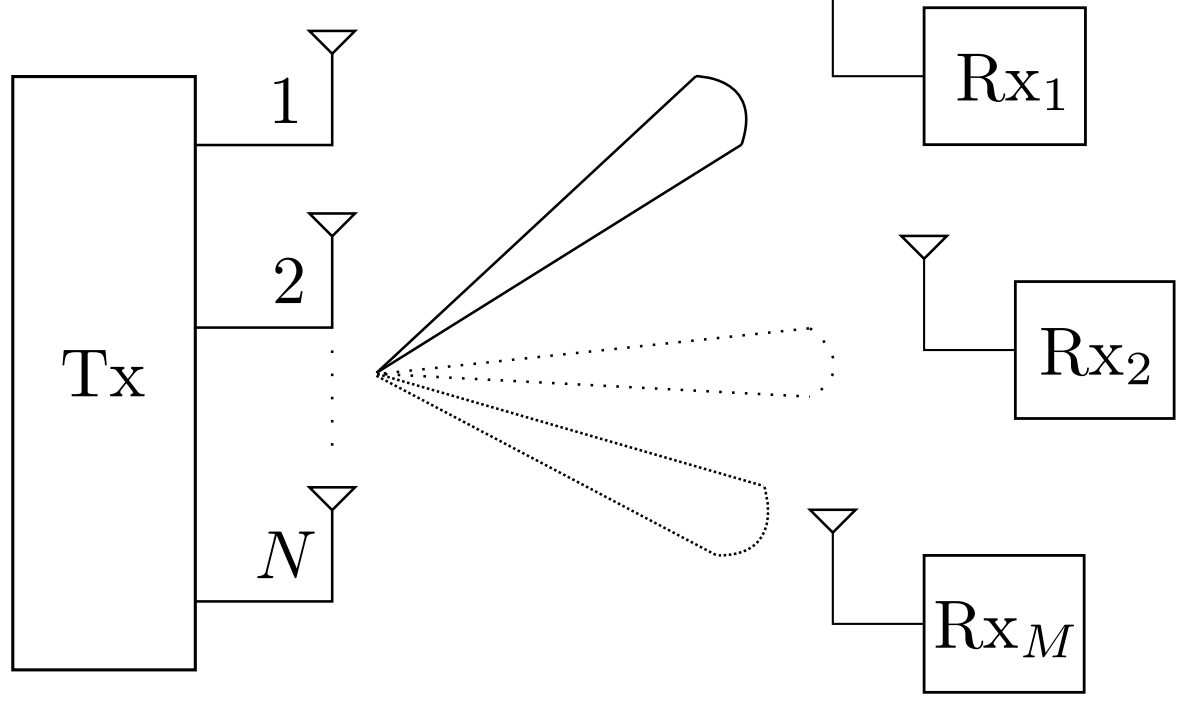

Figure 3.4: Transmit beamformer where one base station transmits to $M$ users.

essential in transmit beamforming. CSIT can be acquired through the reciprocity principle of electromagnetics or through feedback of the channel state information at the receiver (CSIR). Usually CSIT is only an approximation of the true channel. It is well known that some beamforming techniques can be extremely sensitive to channel estimation errors that lead to severe signal cancellation $[25,56]$. This makes the design of transmit beamformers a challenging task.

Fig. 3.4 shows a base station equipped with $N$ transmit antennas simultaneously serving $M$ users, each having a single antenna. The base station transmit signal at time instance $t$ is given by

$$
\mathbf{y}(t)=\sum_{m=1}^{M} s_{m}(t) \mathbf{w}_{m}
$$

where $s_{m}(t)$ and $\mathbf{w}_{m}$ are the $m$ th user's information symbol and beamforming vector, respectively. The signal received at the $m$ th user is given by

$$
r_{m}(t)=\mathbf{h}_{m}^{H} \mathbf{y}(t)+n_{m}(t),
$$

where $\mathbf{h}_{m}$ is the downlink channel vector of the $m$ th user and $n_{m}(t)$ is the additive white Gaussian noise (AWGN) at this receiver. By assuming that the information symbols and the receiver noise are statistically independent, the SINR at the $m$ th user can be expressed as

$$
\gamma_{m}=\frac{\left|\mathbf{w}_{m}^{H} \mathbf{h}_{m}\right|^{2}}{\sum_{l=1, l \neq m}^{M}\left|\mathbf{w}_{l}^{H} \mathbf{h}_{m}\right|^{2}+\sigma_{m}^{2}}
$$

where $\sigma_{m}^{2}$ is the noise power.

The beamformer design problem is commonly formulated as the SINR balancing 
problem [25]. Here, the optimisation problem minimises the total base station transmit power while imposing the constraints that the SINR at each user is above some predefined threshold $\gamma_{m}^{T}$. When the channel vectors $\mathbf{h}_{m}$ are known, this problem is stated as

$$
\begin{aligned}
\min _{\left\{\mathbf{w}_{m}\right\}_{m=1}^{M}} & \sum_{m=1}^{M}\left\|\mathbf{w}_{m}\right\|_{2}^{2} \\
\text { s.t. } & \frac{\left|\mathbf{w}_{m}^{H} \mathbf{h}_{m}\right|^{2}}{\sum_{l=1, l \neq m}^{M}\left|\mathbf{w}_{l}^{H} \mathbf{h}_{m}\right|^{2}+\sigma_{m}^{2}} \geq \gamma_{m}^{T}, \quad m=1, \ldots, M
\end{aligned}
$$

Problem (3.71) is a non-convex optimisation problem; however, by noting that the objective remains unchanged when the beamforming vector undergoes an arbitrary phase shift, the problem can be restated as the following convex quadratic optimisation problem [25]

$$
\begin{array}{cl}
\min _{\left\{\mathbf{w}_{m}\right\}_{m=1}^{M}} & \sum_{m=1}^{M}\left\|\mathbf{w}_{m}\right\|_{2}^{2} \\
\text { s.t. } & \left(\mathbf{w}_{m}^{H} \mathbf{h}_{m}\right)^{2} \geq \gamma_{m}^{T} \sum_{l=1, l \neq m}^{M} \mathbf{w}_{l}^{H} \mathbf{h}_{m} \mathbf{h}_{m}^{H} \mathbf{w}_{l}+\gamma_{m}^{T} \sigma_{m}^{2}, \quad m=1, \ldots, M \\
& \Re\left\{\mathbf{w}_{m}^{H} \mathbf{h}_{m}\right\} \geq 0, \quad m=1, \ldots, M \\
& \Im\left\{\mathbf{w}_{m}^{H} \mathbf{h}_{m}\right\}=0, \quad m=1, \ldots, M
\end{array}
$$

In many practical systems accurate estimates of the instantaneous downlink channel vector $\mathbf{h}_{m}$ may not be available. In these cases, it may be reasonable to assume that the base station has access to the channel correlation matrix $\mathbf{R}_{m}=$ $\mathbb{E}\left\{\mathbf{h}_{m} \mathbf{h}_{m}^{H}\right\}$ [25]. The SINR balancing problem can then be stated as

$$
\begin{aligned}
\min _{\left\{\mathbf{w}_{m}\right\}_{m=1}^{M}} & \sum_{m=1}^{M}\left\|\mathbf{w}_{m}\right\|_{2}^{2} \\
\text { s.t. } & \frac{\mathbf{w}_{m}^{H} \mathbf{R}_{m} \mathbf{w}_{m}}{\sum_{l=1, l \neq m}^{M} \mathbf{w}_{l}^{H} \mathbf{R}_{m} \mathbf{w}_{l}+\sigma_{m}^{2}} \geq \gamma_{m}^{T}, \quad m=1, \ldots, M
\end{aligned}
$$

This is also a non-convex optimisation problem. However, by defining $\mathbf{W}_{m}=$ $\mathbf{w}_{m} \mathbf{w}_{m}^{H}$ and using the SDR concept of Section 2.7.1, the problem can be restated as the following relaxed SDP

$$
\begin{aligned}
\min _{\left\{\mathbf{W}_{m}\right\}_{m=1}^{M}} & \sum_{m=1}^{M} \operatorname{tr}\left(\mathbf{W}_{m}\right) \\
\text { s.t. } & \operatorname{tr}\left(\mathbf{W}_{m} \mathbf{R}_{m}\right) \geq \gamma_{m}^{T} \sum_{l=1, l \neq m}^{M} \operatorname{tr}\left(\mathbf{W}_{l} \mathbf{R}_{m}\right)+\gamma_{m}^{T} \sigma_{m}^{2}, \quad m=1, \ldots, M \\
& \mathbf{W}_{m} \succeq 0, \quad m=1, \ldots, M
\end{aligned}
$$


In [25, Lemma 2], it is shown that the optimum value of problem (3.73) is the same as the optimum value of the Lagrange dual of problem (3.74). This implies that the optimum value of problem (3.73) is the same as the optimum value of problem (3.74). Therefore, problem (3.74) is not a strict relaxation, but actually an equivalent reformulation of problem (3.73). Hence, problem (3.74) does always have at least one optimum solution where all $\mathbf{W}_{m}$ have rank one.

Since downlink beamformers designed based on the assumption of perfect CSI are known to be quite sensitive to channel uncertainties [241], several robust methods have been proposed in the literature [25, 38, 208-210, 239-241]. Imperfections in the channel correlation matrices are considered in [25] and [241] where the channel correlation matrix is modelled as

$$
\mathbf{R}_{m}=\tilde{\mathbf{R}}_{m}+\boldsymbol{\Delta}_{m}, \quad m=1, \ldots, N
$$

Here, $\mathbf{R}_{m}$ and $\tilde{\mathbf{R}}_{m}$ are the actual and the estimated channel correlation matrices of the $m$ th user and $\boldsymbol{\Delta}_{m}$ is the error in the estimate. In [25], the Frobenius norm of the error matrix is assumed to be upper-bounded by a known constant $\epsilon_{m}$, i.e., $\left\|\boldsymbol{\Delta}_{m}\right\| \leq \epsilon_{m}$ and a worst-case SINR constraint is used, i.e.,

$$
\min _{\left\|\boldsymbol{\Delta}_{m}\right\| \leq \epsilon_{m}} \frac{\mathbf{w}_{m}^{H}\left(\tilde{\mathbf{R}}_{m}+\boldsymbol{\Delta}_{m}\right) \mathbf{w}_{m}}{\sum_{l=1, l \neq m}^{M} \mathbf{w}_{l}^{H}\left(\tilde{\mathbf{R}}_{m}+\boldsymbol{\Delta}_{m}\right) \mathbf{w}_{l}+\sigma_{m}^{2}} \geq \gamma_{m}^{T}, \quad m=1, \ldots, M
$$

which is approximated using

$$
\frac{\mathbf{w}_{m}^{H}\left(\tilde{\mathbf{R}}_{m}-\epsilon_{m} \mathbf{I}\right) \mathbf{w}_{m}}{\sum_{l=1, l \neq m}^{M} \mathbf{w}_{l}^{H}\left(\tilde{\mathbf{R}}_{m}+\epsilon_{m} \mathbf{I}\right) \mathbf{w}_{l}+\sigma_{m}^{2}} \geq \gamma_{m}^{T}, \quad m=1, \ldots, M
$$

In [241], the authors argue that constraint (3.77) tends to be overly conservative because the approximation can be very loose and the positive semidefiniteness of the correlation matrices $\tilde{\mathbf{R}}_{m}+\boldsymbol{\Delta}_{m}$ is ignored in the approximation. The robust downlink beamformer in [241] is developed as follows. The $m$ th worst-case constraint (3.76) can be restated as

$$
\min _{\left\|\boldsymbol{\Delta}_{m}\right\| \leq \epsilon_{m}}-\left(\operatorname{tr}\left(\boldsymbol{\Delta}_{m} \mathbf{A}_{m}\right)+\operatorname{tr}\left(\tilde{\mathbf{R}}_{m} \mathbf{A}_{m}\right)+\gamma_{m}^{T} \sigma_{m}^{2}\right) \geq 0
$$

where $\mathbf{A}_{m}=\gamma_{m}^{T} \sum_{l=1, l \neq m}^{M} \mathbf{w}_{l} \mathbf{w}_{l}^{H}-\mathbf{w}_{m} \mathbf{w}_{m}^{H}$. By adding the positive semidefinite constraint on $\tilde{\mathbf{R}}_{m}+\boldsymbol{\Delta}_{m}$, the left hand side of (3.78) can be expressed as the following optimisation problem

$$
\begin{array}{ll}
\min _{\boldsymbol{\Delta}_{m}} & -\left(\operatorname{tr}\left(\boldsymbol{\Delta}_{m} \mathbf{A}_{m}\right)+\operatorname{tr}\left(\tilde{\mathbf{R}}_{m} \mathbf{A}_{m}\right)+\gamma_{m}^{T} \sigma_{m}^{2}\right) \\
\text { s.t. } & \left\|\boldsymbol{\Delta}_{m}\right\| \leq \epsilon_{m}, \\
& -\tilde{\mathbf{R}}_{m}-\boldsymbol{\Delta}_{m} \preceq 0 .
\end{array}
$$


The dual problem associated with problem (3.79) is given by

$$
\begin{aligned}
\max _{\lambda_{m}, \mathbf{Z}_{m}} & -\frac{\left\|\mathbf{A}_{m}+\mathbf{Z}_{m}\right\|^{2}}{4 \lambda_{m}}-\lambda_{m} \epsilon_{m}^{2}-\operatorname{tr}\left(\tilde{\mathbf{R}}_{m}\left(\mathbf{A}_{m}+\mathbf{Z}_{m}\right)\right)-\gamma_{m}^{T} \sigma_{m}^{2} \\
\text { s.t. } & \lambda_{m} \geq 0, \\
& \mathbf{Z}_{m} \succeq 0,
\end{aligned}
$$

where $\lambda_{m}$ and $\mathbf{Z}_{m}$ are the dual variables. Maximising the objective function of (3.80) with respect to $\lambda_{m}$ gives the following problem

$$
\begin{array}{ll}
\max _{\mathbf{Z}_{m}} & -\epsilon_{m}\left\|\mathbf{A}_{m}+\mathbf{Z}_{m}\right\|-\operatorname{tr}\left(\tilde{\mathbf{R}}_{m}\left(\mathbf{A}_{m}+\mathbf{Z}_{m}\right)\right)-\gamma_{m}^{T} \sigma_{m}^{2} \\
\text { s.t. } & \mathbf{Z}_{m} \succeq 0 .
\end{array}
$$

Using (3.81), the robust downlink beamforming problem is expressed as

$$
\begin{aligned}
\min _{\left\{\mathbf{w}_{m}, \mathbf{Z}_{m}\right\}_{m=1}^{M}} & \sum_{m=1}^{M}\left\|\mathbf{w}_{m}\right\|_{2}^{2} \\
\text { s.t. } & -\epsilon_{m}\left\|\mathbf{A}_{m}+\mathbf{Z}_{m}\right\|-\operatorname{tr}\left(\tilde{\mathbf{R}}_{m}\left(\mathbf{A}_{m}+\mathbf{Z}_{m}\right)\right)-\gamma_{m}^{T} \sigma_{m}^{2} \geq 0, \quad m=1, \ldots, M \\
& \mathbf{Z}_{m} \succeq 0, \quad m=1, \ldots, M
\end{aligned}
$$

Through the application of SDR, problem (3.82) can be transformed into an SDP and solved efficiently. Simulation results in [241] show that this beamformer outperforms the design in [25] in terms of transmitted power and feasibility of the robust problem, i.e., this problem tends to be less conservative.

Worst-case robust downlink beamforming designs based on mean square error (MSE) as a QoS parameter have been considered in [210, 239].

Robust downlink beamforming designs based stochastic optimisation approach are the subject of investigation in $[38,208,240]$. In [38], the robust downlink beamformer is formulated based on the outage probability. The authors use the model (3.75) and consider $\boldsymbol{\Delta}_{m}$ to be random matrices. To obtain a mathematically tractable formulation, it is assumed that the real-valued diagonal and the complex-valued upper or lower triangle elements of $\boldsymbol{\Delta}_{m}$ are zero-mean, independent Gaussian values with a variance of $\sigma_{\delta_{m}}^{2}$. The probability of non-outage of the $m$ th user is defined as

$$
P_{m}=\operatorname{Pr}\left\{\frac{\mathbf{w}_{m}^{H}\left(\tilde{\mathbf{R}}_{m}+\boldsymbol{\Delta}_{m}\right) \mathbf{w}_{m}}{\sum_{l=1, l \neq m}^{M} \mathbf{w}_{l}^{H}\left(\tilde{\mathbf{R}}_{m}+\boldsymbol{\Delta}_{m}\right) \mathbf{w}_{l}+\sigma_{m}^{2}} \geq \gamma_{m}^{T}\right\} .
$$

By defining $\mathbf{Z}_{m}=\mathbf{W}_{m}-\gamma_{m}^{T} \sum_{l=1, l \neq m}^{M} \mathbf{W}_{l}$, where $\mathbf{W}_{m}=\mathbf{w}_{m} \mathbf{w}_{m}^{H}$ and $\mathbf{W}_{l}=\mathbf{w}_{l} \mathbf{w}_{l}^{H}$, (3.83) can be restated as

$$
P_{m}=\operatorname{Pr}\left\{\operatorname{tr}\left(\left(\tilde{\mathbf{R}}_{m}+\boldsymbol{\Delta}_{m}\right) \mathbf{Z}_{m}\right) \geq \gamma_{m}^{T} \sigma_{m}^{2}\right\}
$$


Using [38, Lemma 1] and exploiting the fact that the matrix $\tilde{\mathbf{R}}_{m}+\boldsymbol{\Delta}_{m}$ is Hermitian, $\operatorname{tr}\left(\left(\tilde{\mathbf{R}}_{m}+\boldsymbol{\Delta}_{m}\right) \mathbf{Z}_{m}\right)$ is shown to be a real-valued Gaussian random variable with mean and variance equal to $\operatorname{tr}\left(\tilde{\mathbf{R}}_{m} \mathbf{Z}_{m}\right)$ and $\sigma_{\delta_{m}}^{2} \operatorname{tr}\left(\mathbf{Z}_{m} \mathbf{Z}_{m}^{H}\right)$, respectively. Hence, the non-outage probability is given by

$$
P_{m}=\frac{1}{2}+\frac{1}{2} \operatorname{erf}\left(\frac{\operatorname{tr}\left(\tilde{\mathbf{R}}_{m} \mathbf{Z}_{m}\right)-\gamma_{m}^{T} \sigma_{m}^{2}}{\sqrt{2} \sigma_{\delta_{m}}\left\|\mathbf{Z}_{m}\right\|}\right) .
$$

The robust beamformer design problem imposes the constraint that the nonoutage probability of the $m$ th user is above some predefined threshold $p_{m}$, i.e., $P_{m} \geq$ $p_{m}$. Therefore, using (3.85), the non-outage probability constraint is expressed as

$$
\operatorname{tr}\left(\tilde{\mathbf{R}}_{m} \mathbf{Z}_{m}\right)-\gamma_{m}^{T} \sigma_{m}^{2} \geq \sqrt{2} \sigma_{\delta_{m}} \operatorname{erf}^{-1}\left(2 p_{m}-1\right)\left\|\mathbf{Z}_{m}\right\|
$$

Finally, the robust non-outage probability constrained downlink beamforming problem with linear objective function with convex SOC and SDP constraints is stated as the following relaxed SDP

$$
\begin{array}{rlr}
\min _{\left\{\mathbf{W}_{m}\right\}_{m=1}^{M}} & \sum_{m=1}^{M} \operatorname{tr}\left(\mathbf{W}_{m}\right) \\
\text { s.t. } & \operatorname{tr}\left(\tilde{\mathbf{R}}_{m} \mathbf{Z}_{m}\right)-\gamma_{m}^{T} \sigma_{m}^{2} \geq \sqrt{2} \sigma_{\delta_{m}} \operatorname{erf}^{-1}\left(2 p_{m}-1\right)\left\|\mathbf{Z}_{m}\right\|, \\
& & m=1, \ldots, M \\
& \mathbf{W}_{m} \succeq 0, \quad m=1, \ldots, M
\end{array}
$$

\subsubsection{Relay Beamforming}

A relay is typically used in a scenario where the source is unable to directly communicate with the destination due to poor channel conditions. The relay can either amplify-and-forward (AF) or decode-and-forward (DF) the source signal. When the relay node is equipped with multiple antennas then robust receive and transmit beamformers at the relay can be designed using the methods described in Sections 3.3.2 and 3.3.3. The benefits offered by multiple antennas can also be realised by systems employing multiple single antenna relay nodes through a technique known as cooperative relaying [39,68,99, 136, 201,245,269,277]. Geographically distributed relay nodes are cooperatively able to form a virtual antenna array and provide increased gains in capacity through distributed beamforming. In [201,202], it was shown that user cooperation could be used as a form of spatial diversity. This not only resulted in increased capacity for the users but also a more robust system where the users' rates were less affected by channel variations.

Fig. 3.5 shows a wireless relay network which consists of a transmitter, a receiver and $R$ relay nodes. All links in the network are assumed to be flat fading channels. The channel coefficients of the transmitter to the $i$ th relay and the $i$ th 


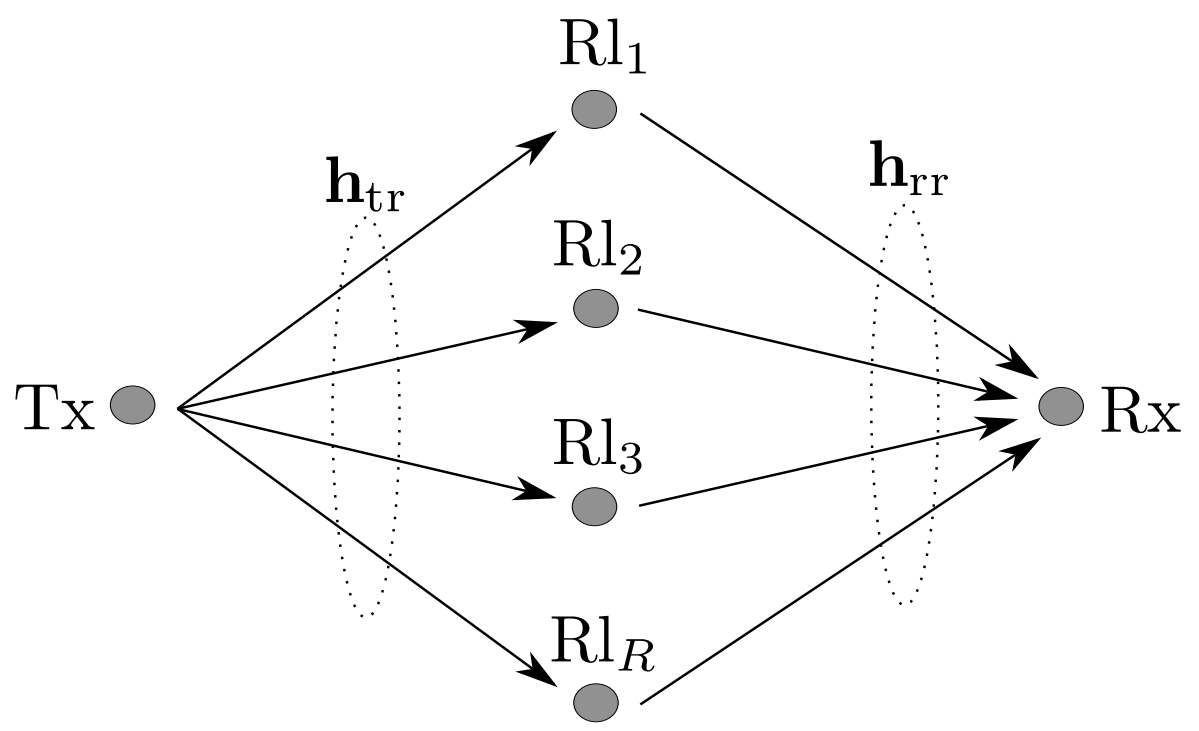

Figure 3.5: Wireless relay network.

relay to the receiver links are denoted by $h_{\mathrm{tr}}^{(i)}$ and $h_{\mathrm{rr}}^{(i)}$, respectively. In [99], robust cooperative relay beamformers are designed based on the knowledge of the secondorder statistics of $h_{\mathrm{tr}}^{(i)}$ and $h_{\mathrm{rr}}^{(i)}$. A two step AF protocol is assumed. During the first step, the transmitter broadcasts the signal $\sqrt{P_{0}} s$ to the relays, where $P_{0}$ is the transmit power and $s$ is the information symbol. It is assumed that $\mathbb{E}\left\{|s|^{2}\right\}=1$. The signal received at the $i$ th relay is given by

$$
x_{i}=\sqrt{P_{0}} s h_{\mathrm{tr}}^{(i)}+n_{\mathrm{r}}^{(i)},
$$

where $n_{\mathrm{r}}^{(i)}$ is the noise at the $i$ th relay with a variance of $\sigma_{\mathrm{r}}^{2}$.

During the second step, the $i$ th relay transmits the signal

$$
\begin{aligned}
y_{i} & =x_{i} w_{i} \\
& =\sqrt{P_{0}} s h_{\mathrm{tr}}^{(i)} w_{i}+n_{\mathrm{r}}^{(i)} w_{i},
\end{aligned}
$$

where $w_{i}$ is the complex beamforming weight applied by the $i$ th relay. At the destination receiver, the received signal can be expressed as

$$
\begin{aligned}
z_{s} & =\sum_{i=1}^{R} y_{i} h_{\mathrm{rr}}^{(i)}+n \\
& =\underbrace{\sqrt{P_{0}} s \sum_{i=1}^{R} h_{\mathrm{tr}}^{(i)} h_{\mathrm{rr}}^{(i)} w_{i}}_{\text {wanted signal }}+\underbrace{\sum_{i=1}^{R} n_{\mathrm{r}}^{(i)} h_{\mathrm{rr}}^{(i)} w_{i}+n}_{\text {noise }},
\end{aligned}
$$

where $n$ is the destination receiver noise with variance $\sigma^{2}$.

In [99], the beamforming weights are obtained in order to either maximise the SINR at the destination receiver subject to some power constraints or keep it above 
a certain threshold while minimising the total transmit power.

First we consider the total relay transmit power minimisation problem. Here, the total relay transmit power, $P_{T}$, is minimised while maintaining the receiver SINR above a threshold $\gamma$. This problem is stated as [99]

$$
\begin{aligned}
\min _{\left\{w_{i}\right\}_{i=1}^{R}} & P_{T} \\
\text { s.t. } & \text { SINR } \geq \gamma .
\end{aligned}
$$

The total relay transmit power is given by

$$
\begin{aligned}
P_{T} & =\sum_{i=1}^{R} \mathbb{E}\left\{\left|y_{i}\right|^{2}\right\} \\
& =\mathbf{w}^{H} \mathbf{D} \mathbf{w},
\end{aligned}
$$

where $\mathbf{w} \triangleq\left[w_{1} w_{2} \ldots w_{R}\right]^{T}$ and $\mathbf{D} \triangleq P_{0} \operatorname{diag}\left(\left[\mathbb{E}\left\{\left|h_{\mathrm{tr}}^{(1)}\right|^{2}\right\} \mathbb{E}\left\{\left|h_{\mathrm{tr}}^{(2)}\right|^{2}\right\} \ldots \mathbb{E}\left\{\left|h_{\mathrm{tr}}^{(R)}\right|^{2}\right\}\right]\right)+$ $\sigma_{\mathrm{r}}^{2} \mathbf{I}$. The wanted signal power is given by

$$
\begin{aligned}
P_{s} & =\mathbb{E}\left\{\left|\sum_{i=1}^{R} w_{i} h_{\mathrm{tr}}^{(i)} h_{\mathrm{rr}}^{(i)}\right|^{2}|s|^{2}\right\} \\
& =\mathbf{w}^{H} \mathbf{R} \mathbf{w},
\end{aligned}
$$

where $\mathbf{R} \triangleq P_{0} \mathbb{E}\left\{\left(\mathbf{h}_{\mathrm{tr}} \odot \mathbf{h}_{\mathrm{rr}}\right)\left(\mathbf{h}_{\mathrm{tr}} \odot \mathbf{h}_{\mathrm{rr}}\right)^{H}\right\}, \mathbf{h}_{\mathrm{tr}}=\left[h_{\mathrm{tr}}^{(1)} h_{\mathrm{tr}}^{(2)} \ldots h_{\mathrm{tr}}^{(R)}\right]^{T}, \mathbf{h}_{\mathrm{rr}}=\left[h_{\mathrm{rr}}^{(1)} h_{\mathrm{rr}}^{(2)} \ldots h_{\mathrm{rr}}^{(R)}\right]^{T}$ and $\odot$ is the element-wise Schur-Hadamard product. The total noise power at the destination receiver is expressed as

$$
\begin{aligned}
P_{n_{T}} & =\mathbb{E}\left\{\sum_{i=1}^{R} \sum_{j=1}^{R} w_{i} w_{j}^{*} h_{\mathrm{rr}}^{(i)} h_{\mathrm{rr}}^{(j)}\right\} \mathbb{E}\left\{\left|n_{\mathrm{r}}^{(i)}\right|^{2}\right\}+\mathbb{E}\left\{|n|^{2}\right\} \\
& =\mathbf{w}^{H} \mathbf{Q} \mathbf{w}+\sigma^{2},
\end{aligned}
$$

where $\mathbf{Q} \triangleq \sigma_{r}^{2} \mathbb{E}\left\{\mathbf{h}_{\mathrm{rr}}\right\}$. Using (3.92), (3.93) and (3.94), problem (3.91) can be stated as $[99]$

$$
\begin{array}{ll}
\min _{\mathbf{w}} & \mathbf{w}^{H} \mathbf{D} \mathbf{w} \\
\text { s.t. } & \frac{\mathbf{w}^{H} \mathbf{R w}}{\mathbf{w}^{H} \mathbf{Q} \mathbf{w}+\sigma^{2}} \geq \gamma .
\end{array}
$$

By defining $\tilde{\mathbf{w}}=\mathbf{D}^{1 / 2} \mathbf{w}$, problem (3.95) can be restated as

$$
\begin{array}{ll}
\min _{\tilde{\mathbf{w}}} & \|\tilde{\mathbf{w}}\|_{2}^{2} \\
\text { s.t. } & \tilde{\mathbf{w}}^{H} \mathbf{D}^{-1 / 2}(\mathbf{R}-\gamma \mathbf{Q}) \mathbf{D}^{-1 / 2} \tilde{\mathbf{w}} \geq \gamma \sigma^{2} .
\end{array}
$$

As stated by the authors in [99], the constraint in (3.96) is satisfied with equality at 
the optimum, for otherwise, the optimal $\tilde{\mathbf{w}}$ could be scaled down to satisfy the constraint with equality, thereby decreasing the objective function and contradicting optimality. The Lagrangian function of (3.96) can therefore be written as

$$
L(\tilde{\mathbf{w}}, \lambda)=\tilde{\mathbf{w}}^{H} \tilde{\mathbf{w}}-\lambda\left(\tilde{\mathbf{w}}^{H} \mathbf{D}^{-1 / 2}(\mathbf{R}-\gamma \mathbf{Q}) \mathbf{D}^{-1 / 2} \tilde{\mathbf{w}}-\gamma \sigma^{2}\right)
$$

and the derivative of the Lagrangian function with respect to $\tilde{\mathbf{w}}$ as

$$
\frac{\partial L(\tilde{\mathbf{w}}, \lambda)}{\partial \tilde{\mathbf{w}}}=2 \tilde{\mathbf{w}}-2 \lambda \mathbf{D}^{-1 / 2}(\mathbf{R}-\gamma \mathbf{Q}) \mathbf{D}^{-1 / 2} \tilde{\mathbf{w}}
$$

The KKT conditions for optimality, i.e., the gradient of the Lagrangian vanishes at the optimum and primal feasibility, are given by

$$
\begin{aligned}
\mathbf{D}^{-1 / 2}(\mathbf{R}-\gamma \mathbf{Q}) \mathbf{D}^{-1 / 2} \tilde{\mathbf{w}}^{*} & =\frac{1}{\lambda} \tilde{\mathbf{w}}^{*} \\
\tilde{\mathbf{w}}^{* H} \mathbf{D}^{-1 / 2}(\mathbf{R}-\gamma \mathbf{Q}) \mathbf{D}^{-1 / 2} \tilde{\mathbf{w}}^{*} & =\gamma \sigma^{2} .
\end{aligned}
$$

We see that (3.99a) is an eigenvalue problem and $\tilde{\mathbf{w}}^{*}$ is an eigenvector of $\mathbf{D}^{-1 / 2}(\mathbf{R}-\gamma \mathbf{Q}) \mathbf{D}^{-1 / 2}$ and $1 / \lambda$ the corresponding eigenvalue. Using (3.99a) and (3.99b), the optimum value of the objective function of (3.96) is given by

$$
\left\|\tilde{\mathbf{w}}^{*}\right\|_{2}^{2}=\lambda \gamma \sigma^{2}
$$

This implies that in order to minimise $\|\tilde{\mathbf{w}}\|_{2}^{2}, \lambda$ should be minimised. Hence, $1 / \lambda$ is selected to be the largest eigenvalue of $\mathbf{D}^{-1 / 2}(\mathbf{R}-\gamma \mathbf{Q}) \mathbf{D}^{-1 / 2}[99]$. Finally, the optimum beamforming vector is given by [99]

$$
\mathbf{w}^{*}=\left(\frac{\gamma \sigma^{2}}{\mathbf{u}^{H} \mathbf{D}^{-1 / 2}(\mathbf{R}-\gamma \mathbf{Q}) \mathbf{D}^{-1 / 2} \mathbf{u}}\right)^{1 / 2} \mathbf{D}^{-1 / 2} \mathbf{u}
$$

where $\mathbf{u}$ is the normalised principal eigenvector of $\mathbf{D}^{-1 / 2}(\mathbf{R}-\gamma \mathbf{Q}) \mathbf{D}^{-1 / 2}$.

The second beamformer design problem considered in [99] is the maximisation of the destination receiver SINR subject to individual relay power constraints. This optimisation problem is expressed as

$$
\begin{array}{ll}
\min _{\mathbf{w}} & \frac{\mathbf{w}^{H} \mathbf{R} \mathbf{w}}{\mathbf{w}^{H} \mathbf{Q} \mathbf{w}+\sigma^{2}} \\
\text { s.t. } & \mathbf{D}_{i i}\left|w_{i}\right|^{2} \leq P_{i}, \quad i=1,2, \ldots, R
\end{array}
$$

where $\mathbf{D}_{i i}$ is the $i$ th diagonal entry of the matrix D. Problem (3.102) is a nonconvex optimisation problem; however, it can be transformed into a convex form as described next. Using the definition $\mathbf{W} \triangleq \mathbf{w} \mathbf{w}^{H}$, problem (3.102) can be restated 
as the following SDP

$$
\begin{array}{ll}
\min _{\mathbf{W}} & \frac{\operatorname{tr}(\mathbf{R W})}{\operatorname{tr}(\mathbf{Q W})+\sigma^{2}} \\
\text { s.t. } & \mathbf{D}_{i i} \mathbf{W}_{i i} \leq P_{i}, \quad i=1,2, \ldots, R \\
& \mathbf{W} \succeq 0, \\
& \operatorname{rank}(\mathbf{W})=1 .
\end{array}
$$

Using the epigraph form, problem (3.103) can be written as [99]

$$
\begin{array}{ll}
\min _{\mathbf{W}, t} & t \\
\text { s.t. } & \operatorname{tr}(\mathbf{W}(\mathbf{R}-t \mathbf{Q})) \geq t \sigma^{2} \\
& \mathbf{D}_{i i} \mathbf{W}_{i i} \leq P_{i}, \quad i=1,2, \ldots, R \\
& \mathbf{W} \succeq 0, \\
& \operatorname{rank}(\mathbf{W})=1 .
\end{array}
$$

Due to the non-convex constraints (3.104b) and (3.104e), problem (3.104) is a non-convex optimisation problem. To proceed, the authors of [99] apply the idea of SDR and relax the problem by removing the rank constraint (3.104e). The resulting relaxed problem is still non-convex as constraint (3.104b) remains to be dealt with. In $[29,99]$, it was shown that in problems of this nature, for any fixed value of $t$ the set of feasible $\mathbf{W}$ is convex and hence the relaxed problem is quasi convex. Therefore, for some given $t$, problem (3.104) can be expressed as the following convex feasibility problem

$$
\begin{array}{ll}
\text { find } & \mathbf{W} \\
\text { s.t. } & \operatorname{tr}(\mathbf{W}(\mathbf{R}-t \mathbf{Q})) \geq t \sigma^{2} \\
& \mathbf{D}_{i i} \mathbf{W}_{i i} \leq P_{i}, \quad i=1,2, \ldots, R \\
& \mathbf{W} \succeq 0 .
\end{array}
$$

The bisection method [29, p. 146] is a commonly used technique for solving convex feasibility problems like problem (3.105). Upon completion of the bisection algorithm, the optimum beamforming vector, $\mathbf{w}^{*}$, needs to be recovered from $\mathbf{W}$. Since problem (3.105) is a relaxed form of the original problem, $\mathbf{W}$ is not guaranteed to be rank one. In [99], the principal eigenvector of $\mathbf{W}$ is chosen as the optimum beamforming vector when $\mathbf{W}$ happens to be rank one. When $\mathbf{W}$ is not rank one, the Gaussian sampling technique discussed in Section 2.7.3 can be used to obtain a good approximation of a rank 1 solution. In [99], it is reported that in their extensive numerical simulations, the authors never encountered a case where the solution had a rank higher than one. 


\subsection{Summary}

This chapter established the theory and application of robust optimisation to communication systems. Robust optimisation is generally used when the input data has some degree of uncertainty. This occurs frequently in many real world engineering problems and robust optimisation methods seek to find solutions that offer robustness against the uncertainty. Robust optimisation techniques are generally based on bounded uncertainty models where bounds on data uncertainty are known or stochastic optimisation where the statistics of the uncertain data are known.

Bounded uncertainty based optimisation offers hard performance guarantees, i.e., constraints are guaranteed to be met in the worst-case scenario; however, very conservative solutions are usually obtained. The ellipsoidal uncertainty model is the most commonly used uncertainty model because an ellipsoid has a simple mathematical representation and easily handled numerically, ellipsoids and intersections of ellipsoids can be used to more complicated uncertainty sets and in some cases where the uncertain data has an underlying stochastic model, the stochastic uncertainty can be replaced by a deterministic ellipsoidal uncertainty.

Stochastic optimisation methods utilise the knowledge of the statistics of the uncertain data to obtain solutions that offer some average performance or performance that meets design requirements with a given probability. Solutions obtained through stochastic optimisation methods tend to be less conservative than bounded uncertainty based methods.

Applications of bounded uncertainty based optimisation and stochastic optimisation in communication systems, specifically robust power control, robust conventional receive and transmit beamforming and cooperative beamforming, was outlined. 


\section{Chapter 4}

\section{Cognitive Radio Systems}

In recent years, the explosive growth in the use of wireless devices has made the problem of spectrum utilisation more critical. Governments have traditionally adopted a fixed spectrum access policy whereby bands of spectrum are exclusively licensed to one or more dedicated users. This creates a situation where only the licensed user is allowed to use the spectrum even when the spectrum is unused. Due to this usage model and the finite availability of spectrum, most of the available spectrum has been fully allocated in several countries [138] and the problem of spectrum scarcity is beginning to appear.

In 2002, a report aimed at improving the manner in which spectrum is managed in the United States was published by the FCC Spectrum Policy Task Force [48]. One of the main findings of this report was that in many bands, spectrum access rather than the physical scarcity of spectrum is a significant problem. It was identified that the challenges to spectrum access were largely brought on by legacy command-and-control regulation that limits the ability of potential spectrum users to obtain such access. The FCC findings are supported by various measurements of spectrum utilisation $[60,112,130,158,159,220]$. These measurements have revealed that, even in urban areas, there are substantial unused resources in frequency, time and space. In [130], this underutilised spectrum is referred to as spectrum holes, i.e., a band of frequencies assigned to a primary (licensed) user (PU), but, at a particular time and specific geographic location, the band is not being used by that user. To maintain sustainable wireless services, new methods of spectrum utilisation and policy changes are needed. Clearly, if secondary (unlicensed) users (SU) are allowed to access spectrum holes unoccupied by the PUs, then spectrum utilisation can be significantly improved.

CR $[163,164]$ is a new paradigm for exploiting underutilised spectral resources by reusing unused spectrum in a dynamic and opportunistic manner. This access method is commonly referred to as dynamic spectrum access (DSA). In [102], a $\mathrm{CR}$ is defined as an intelligent wireless communication system that is aware of its surrounding environment and adapts its internal states to statistical variations in 
the incoming RF stimuli by making corresponding changes in certain operating parameters, such as carrier frequency, bandwidth, modulation, transmit power and beamformer parameters, in real time. In order to achieve its goals, a cognitive radio undertakes the following fundamental tasks, known as a cognitive cycle [102, 164]

1. Radio scene analysis, which involves estimation of interference level and detection of spectrum holes.

2. Channel estimation and channel capacity prediction.

3. Transceiver configuration, which may involve carrier frequency and bandwidth selection, transmit power control, modulation selection and beamformer weight selection.

Two basic spectrum access methods for CRs have been proposed in the literature [90, 138, 164, 272, 275]: Opportunistic Spectrum Access (OSA) (also referred to as interweave) and Spectrum Sharing (SS) (also referred to as underlay or concurrent spectrum access). In the OSA model, the SUs are allowed to transmit in the band of interest when none of the PUs are transmitting in that band. This is achieved through spectrum sensing $[1,13,103,138,150,257,265]$ where the SUs detect active PU signals in the band of interest. By contrast, the SS model allows the SUs to transmit concurrently with the PUs in the same band of interest, provided that the SUs are able to maintain performance degradation at the PU receivers within some acceptable margin.

The recent advancements in CR technologies have led the IEEE to finalise the 802.22 wireless regional area network (WRAN) standard [94]. This is the first worldwide standard aimed at utilising the white spaces in the television (TV) spectrum for broad-band access in rural areas using CR technology.

The rest of this chapter presents an overview of the various spectrum sensing and spectrum sharing techniques from the literature.

\subsection{Spectrum Sensing for OSA}

In the cognitive cycle of the OSA model, spectrum sensing forms the main part of the radio scene analysis. The aim of spectrum sensing is to robustly and reliably detect whether the band of interest is available or not. The basic idea of spectrum sensing is to discriminate between two hypotheses: $\mathcal{H}_{0}$ for PU signal absent and $\mathcal{H}_{1}$ for PU signal present. This is mathematically represented as [13]

$$
\begin{aligned}
\mathcal{H}_{0}: \mathbf{y}[k] & =\mathbf{n}[k], & k & =1, \ldots, K \\
\mathcal{H}_{1}: \mathbf{y}[k] & =\mathbf{x}[k]+\mathbf{n}[k], & k & =1, \ldots, K,
\end{aligned}
$$


where $\mathbf{x}[k] \in \mathbb{C}^{M \times 1}$ is the PU's signal at the SU receiver, $\mathbf{n}[k] \in \mathbb{C}^{M \times 1}$ represents the $\mathrm{SU}$ receiver noise, $k$ represents time and $\mathbf{y}[k] \in \mathbb{C}^{M \times 1}$ is the baseband signal at the SU receiver. The $m$ th element of $\mathbf{y}[k]$ could represent the received signal at the $m$ th antenna. In the literature $[13,150,265]$, it is common to stack the vectors in (4.1) to form vectors of length $M K$. Using this gives the following compact representation of (4.1)

$$
\begin{aligned}
& \mathcal{H}_{0}: \mathbf{y}=\mathbf{n} \\
& \mathcal{H}_{1}: \mathbf{y}=\mathbf{x}+\mathbf{n} .
\end{aligned}
$$

In (4.2), $\mathbf{n}$ is assumed to be a zero-mean vector with complex Gaussian entries and the covariance matrix $\sigma^{2} \mathbf{I}$.

In order to determine whether $\mathbf{y}$ is generated under $\mathcal{H}_{0}$ or $\mathcal{H}_{1}$, a test statistic, $\Lambda(\mathbf{y})$, is generated from the received signal $\mathbf{y}$ and compared to a predefined threshold $\gamma[125,178,198]$, i.e.,

$$
\Lambda(\mathbf{y}) \underset{\mathcal{H}_{0}}{\stackrel{\mathcal{H}_{1}}{\gtrless}} \gamma
$$

For a detector, the probability of detection is defined as the probability that the test statistic is greater than the threshold given hypothesis $\mathcal{H}_{1}$, i.e., $P_{D}=$ $\operatorname{Pr}\left(\Lambda(\mathbf{y})>\gamma \mid \mathcal{H}_{1}\right)$. Similarly, the probability of false alarm is defined as the probability that the test statistic is greater than the threshold given hypothesis $\mathcal{H}_{0}$, i.e., $P_{F A}=\operatorname{Pr}\left(\Lambda(\mathbf{y})>\gamma \mid \mathcal{H}_{0}\right)$.

In order to obtain good detection performance, $\Lambda(\mathbf{y})$ and $\gamma$ need to be chosen carefully. Either a classical (deterministic) or Bayesian framework can be used to design a detector. The classical framework uses the Neyman-Pearson theorem $[125,178,198]$ for detector design. The Neyman-Pearson theorem states that for a given probability of false alarm, the test statistic that maximises the probability of detection is the likelihood ratio test (LRT) given by

$$
\Lambda(\mathbf{y})=\frac{f\left(\mathbf{y} \mid \mathcal{H}_{1}\right)}{f\left(\mathbf{y} \mid \mathcal{H}_{0}\right)},
$$

where $f(\cdot)$ denotes the PDF. A drawback of using the LRT is that it requires the knowledge of the exact probability distributions of the source signal, the wireless channel and the noise, which is difficult to realise in practice.

In the Bayesian framework, it is assumed that the source selects the true hypothesis at random according to some a priori probabilities $\operatorname{Pr}\left(\mathcal{H}_{0}\right)$ and $\operatorname{Pr}\left(\mathcal{H}_{1}\right)$ [198]. The aim is to marginalise the likelihood function needed in the LRT to eliminate 
the unknowns, i.e.,

$$
f\left(\mathbf{y} \mid \mathcal{H}_{0}\right)=\int f\left(\mathbf{y} \mid \mathcal{H}_{0}, \Theta_{0}\right) f\left(\Theta_{0} \mid \mathcal{H}_{0}\right) \mathrm{d} \Theta_{0}
$$

where $\Theta_{0}$ represents all unknown parameters when $\mathcal{H}_{0}$ is true, $f\left(\mathbf{y} \mid \mathcal{H}_{0}, \Theta_{0}\right)$ denotes the conditional PDF of $\mathbf{y}$ under $\mathcal{H}_{0}$ and conditioned on $\Theta_{0}$, and $f\left(\Theta_{0} \mid \mathcal{H}_{0}\right)$ is the a priori probability distribution of $\Theta_{0}$ under $\mathcal{H}_{0}$. The same analysis applies to hypothesis $\mathcal{H}_{1}$. The main drawbacks of the Bayesian approach is that it requires the knowledge of the a priori distribution of the unknowns under each of the two hypotheses. This is generally not perfectly known but nevertheless can be chosen to provide a meaningful result. The choice of prior distributions affects the detection performance dramatically and therefore, this is not a trivial task [265].

In order to use the LRT, the unknown parameters need to be estimated. It is common to use the maximum-likelihood (ML) estimates of the unknown parameters, which gives rise to the generalised likelihood-ratio test (GLRT)

$$
\frac{\max _{\Theta_{1}} f\left(\mathbf{y} \mid \mathcal{H}_{1}, \Theta_{1}\right)}{\max _{\Theta_{0}} f\left(\mathbf{y} \mid \mathcal{H}_{0}, \Theta_{0}\right)} \underset{\mathcal{H}_{0}}{\stackrel{\mathcal{H}_{1}}{\gtrless}} \gamma .
$$

\subsubsection{Energy Detection}

Energy detection $[63,125,194,206,217,233]$ is the simplest form of spectrum sensing. An energy detector (ED) does not need any knowledge of the PU's signal and therefore is robust against the variation of the PU's signal. It treats the PU's signal as noise and makes a decision on whether the PU's signal is present or not based on the energy of the received signal. The ED is designed by assuming that the received PU's signal is zero-mean circularly symmetric complex Gaussian, i.e., $\mathbf{x} \sim \mathcal{N}_{\mathcal{C}}\left(\mathbf{0}, \xi^{2} \mathbf{I}\right)$. Hence, $\mathbf{y} \mid \mathcal{H}_{0} \sim \mathcal{N}_{\mathcal{C}}\left(\mathbf{0}, \sigma^{2} \mathbf{I}\right)$ and $\mathbf{y} \mid \mathcal{H}_{1} \sim \mathcal{N}_{\mathcal{C}}\left(\mathbf{0},\left(\sigma^{2}+\xi^{2}\right) \mathbf{I}\right)$. The Neyman-Pearson LRT is therefore given by [125]

$$
\Lambda(\mathbf{y})=\frac{\|\mathbf{y}\|_{2}^{2}}{\sigma^{2}}=\underset{\mathcal{H}_{0}}{\stackrel{\mathcal{H}_{1}}{\gtrless}} \gamma .
$$

In [108], it was shown that ED is the optimal detector if only the noise power is known to the SUs. The ED suffers from a number of drawbacks, some of which include i) an inability to distinguish different types of signals, which increases the probability of false alarm when the received signal contains unintended interference [138]; ii) a susceptibility to the uncertainty of noise power, which makes threshold selection difficult [229]; and iii) poor performance in detecting spread spectrum signals $[34,256]$. 


\subsubsection{Matched Filter Detection}

Wireless systems usually employ pilots, preambles or training sequences to aid synchronisation and channel estimation. When these patterns are known to the SUs, coherent matched filter detection can be used for spectrum sensing $[108,162$, 230]. This involves correlating the received signal with a known copy of itself. In this case, the optimal test statistic is the output of the matched filter $[125,178]$, i.e.,

$$
\Re\left(\mathbf{x}^{H} \mathbf{y}\right) \underset{\mathcal{H}_{0}}{\stackrel{\mathcal{H}_{1}}{\gtrless}} \gamma
$$

In [230], it was shown that matched filter based spectrum sensing outperforms ED in reliability and sensing time. However, the estimation error can be large in low SNR scenarios [138].

\subsubsection{Sensing Based on Feature Detection}

Practical communication signals usually contain distinctive features that can be used for detection. Unknown parameters can also be estimated from known signal features $[13,138]$. These features generally originate from modulation, coding and burst formatting techniques used in modern communication systems [13]. For example, orthogonal frequency division multiplexing (OFDM) modulation prepends a cyclic prefix (CP) of length $N_{C}$ to every transmitted OFDM symbol to mitigate the effects of multipath propagation. The CP is chosen to be the last $N_{C}$ samples of the OFDM symbol, hence, the first and last $N_{C}$ samples of the OFDM symbol will be highly correlated. To aid parameter tracking, most communication systems transmit pilots and this also introduces distinctive signal features. Spacetime coding introduces correlation into the transmitted signal which can result in distinguishable signal features. In a MIMO system, if there are more receiving antennas than transmitting, then the received signal will be correlated $[13,138]$.

This section briefly describes detectors that exploit known features in the PU's signal. We focus on detectors based on second-order statistics and those based on cyclostationarity.

\section{Detectors Based on Second-Order Statistics}

Communication systems typically introduce redundancy into the transmitted samples, hence, the received samples are correlated. The CP in OFDM modulation discussed above is one such example. The second-order statistics can therefore be used to distinguish a white signal from a coloured one. Communication signals are generally zero-mean and very nearly Gaussian distributed and therefore the second-order statistics are sufficient for detection. 
Detectors based on second-order statistics of OFDM signals have been considered in $[11,43,134]$. Assume that a single receive antenna is utilised and the OFDM symbols contain $N_{\mathrm{d}}$ data symbols and $N_{\mathrm{c}}$ CP symbols. The SU collects a number of consecutive OFDM symbols and forms the following auto-correlation function $(\mathrm{ACF})$ of the received signal

$$
r_{\mathbf{y}}[k, l]=\sum_{m=0}^{N_{\mathrm{c}}-1} \mathbf{y}[k+m] \mathbf{y}^{*}[k+m+l],
$$

where $l$ is the correlation lag. Since the ACF of white noise is zero for all lags $l \neq 0$, the ACF will be zero under hypothesis $\mathcal{H}_{0}$. Due to the CP in the OFDM symbols, the AFC will be nonzero for lag $l=N_{\mathrm{d}}$ for some time instances $k$ and zero for others. Hence, the ACF is time-varying under hypothesis $\mathcal{H}_{1}$. Several different statistical tests to detect the PU's signal have been proposed in $[11,43,134]$. All of these exploit the non-stationary property of the ACF magnitude.

\section{Detectors Based on Cyclostationarity}

Many man-made signals are not only non-stationary, but also cyclostationary, i.e., their statistics such as mean or auto-correlation, exhibit periodicity. This may arise due to modulation and coding, pilots for channel estimation and synchronisation or even can be intentionally induced to aid spectrum sensing [155,226, 227]. For example, due to the $\mathrm{CP}$, the ACF of the OFDM modulated signal is periodic with period $N_{\mathrm{c}}+N_{\mathrm{d}}$. Detectors based on cyclostationarity detect PU transmissions by exploiting the cyclostationarity of the received signals $[34,58,86,97,126,145,180$, 206].

Cyclostationary detection requires the computation of the cyclic spectrum density function (CSD) of the received signal as [80]

$$
S(f, \alpha)=\sum_{l=-\infty}^{\infty} R_{y}^{\alpha}(l) \exp (-j 2 \pi f l),
$$

where

$$
R_{\mathbf{y}}^{\alpha}(l)=\mathbb{E}\left[\mathbf{y}(k) \mathbf{y}^{*}(k-l) \exp (-j 2 \pi \alpha k)\right]
$$

is the cyclic auto-correlation function $(\mathrm{CAF})$ and $\alpha$ is the cyclic frequency. When the cyclic frequency is equal to the fundamental frequencies of the transmitted signal, the CSD function outputs peak values. Since white noise does not have any cyclostationarity, i.e., its CSD has zero values for all non-zero cyclic frequencies, it is possible to distinguish noise from the PU's signal. Furthermore, different signal types may have different non-zero cyclic frequencies, hence, it is also possible to distinguish between different transmitted signals. 
The cyclostationary features do not vary with the SNR, therefore, detectors based on cyclostationarity can work in very low SNRs [44]. The main drawback of cyclostationarity based detection is its high computational complexity compared with ED and matched filter detection [138].

\subsubsection{Blind Detection}

When the SUs have no information about the PU's transmitted signal, such as the waveform or the cyclic frequency, blind detection methods need to be used. In this section, we briefly describe a number of blind detection methods.

\section{Covariance Based Detection (CBD)}

In practical wireless systems, the received signal is generally temporally correlated due to the dispersive nature of the wireless channel. This temporal correlation in the received signal can be used to differentiate the PU's signal from noise. Covariance based detectors $[262,264]$ determine the presence or absence of the PU's signal based on the covariance of the received signal. The significance of the correlations of the received signal with non-zero lags compared to the correlation with zero lag specifies the presence or absence of the PU's signal.

\section{Sensing Using Multiple Antennas}

Eigenvalue based detection (EBD) for spectrum sensing can be used when the SU receivers are equipped with multiple antennas [10,51,244,261,263, 273]. The maximum and minimum eigenvalues of the received sample covariance matrix can be used to detect the presence of the PU's signal. When the PU's signal is not present, the two eigenvalues will generally have similar magnitudes; however, when the PU's signal is present and the sample covariance matrix is not a multiple of the identity matrix, the difference between the maximum and minimum eigenvalues is expected to be large [138]. Therefore, the condition number of the sample covariance matrix can be used as the test statistic for signal detection [261,263].

Furthermore, in [12], it was shown that some communication signals impose a specific known structure to the sample covariance matrix, hence, the eigenvalue structure of the covariance matrix will be different based on the communication signal type. For instance, single-input multiple output (SIMO), MIMO, orthogonal space-time block code (OSTBC) and OFDM exhibit very different eigenvalue structures [13]. This can be used by the SUs for both detection and identification of signalling type used by the PUs. 


\section{Wideband Spectrum Sensing}

In many cognitive radio applications, a wide band of spectrum needs to be sensed. One method of performing this task is to divide the band into a number of narrowband channels and individually or jointly sense on these channels. This is known as multiband sensing [13]. A simple approach is to assume that the subchannels are independent and perform individual sensing using the blind methods discussed above.

In practice, both the PU's signal and the noise variance can be correlated across the subchannels $[9,107]$. In this case, joint detection can be performed across the subchannels; however, the detection complexity grows exponentially with the number of channels $[9,107]$. In [52-54], a computationally tractable blind wideband interference detection algorithm using a hidden Markov model (HMM) is proposed.

\section{$4.2 \quad$ Spectrum Sharing}

As discussed previously, in the SS or underlay paradigm, the SUs are allowed to access the wireless channel concurrently with the PUs as long as the primary system's performance is not significantly degraded. The development of effective spectrum sharing methods is the main subject of this thesis.

One of the approaches for safeguarding the PU is based on the concept of interference-temperature limit [48]. The interference-temperature is defined as the total interference power at a PU receiver and the limit serves as a "cap" on the maximum interference level that be tolerated by the PU receiver. One of the main drawbacks of the constant interference-temperature based protection is that it does not take into account the PU transmitter to PU receiver link quality into account, hence, the interference limit cannot be adapted accordingly. For instance, when the PU transmitter to PU receiver link is strong, the PU receiver is able to tolerate a higher level of interference compared to when this link is weak. To overcome this shortcoming, a PU receiver SINR-based protection mechanism has been proposed in the literature $[65,214,224,225]$. This scheme has the advantage that it can adapt to the PU receiver to PU transmitter link thus benefiting the SU when this link is strong. However, it imposes additional CSI requirements [225] on the SU and increased collaboration with the primary system is needed. In this thesis, the SINR-based protection mechanism is adopted in the development of the spectrum sharing methods.

SS-based systems can be mainly classified into two categories [111,242] according to their: i) architecture; and ii) spectrum allocation behaviour. The architecture could be either centralised $[32,260]$ or distributed $[109,151,181]$. In centralised spectrum sharing, a central controller controls the spectrum allocation and access procedures. Distributed nodes generally forward CSI and spectrum measurements 
to the central controller to aid spectrum allocation. Distributed spectrum sharing is used in situations where construction of an infrastructure may not be possible. Here, the nodes are responsible for spectrum allocation and access is generally based on local policies. The spectrum allocation behaviour can be either cooperative $[32,109]$ or non-cooperative $[181,278]$. Cooperative methods consider the effect of each node's communication on other nodes. Measurements made by each node is shared among other nodes and this information is used in spectrum allocation. In non-cooperative solutions, the nodes work independently of each other and each node tries to achieve its goals without consideration of other nodes. This may result in reduced spectrum utilisation; however, the communication requirements among the nodes are much lower compared to cooperative solutions.

The optimal approach to designing SS-based systems would be to consider the $\mathrm{PU}$ and SU systems as one large interference network and jointly optimise their transmissions to maximise the SU system's throughput while guaranteeing QoS to the PU system [271]. However, in practice, the PU systems are designed independently from the SU systems, since the PU and SU systems may belong to different operators or the PU systems may already exist. Hence, the SU systems would generally be designed independently with some knowledge of the PU systems. The fundamental information theoretic capacity limits of such systems have been analysed in $[65,85,114,118,170,225,243]$.

Two efficient spectrum access methods can be used in the underlay paradigm. When the SU transmitters and receivers are equipped with single antennas, transmit power control can be utilised. Transmit beamforming can be used when the SU transmitters are equipped with multiple antennas. These two methods are discussed next.

\subsubsection{Transmit Power Control}

As discussed in Section 3.3.1, transmit power control provides a method for controlling interference and increased utilisation of the wireless spectrum in multi-user communication systems where the users access the shared channel simultaneously. The same concept can be applied to a SS-based system to manage the interference among the SUs and to avoid harmful interference to the PUs.

The transmit power control for underlay CR systems is considered in Chapter 5.

\subsubsection{Transmit Beamforming}

Conventional and cooperative transmit beamforming were discussed in Sections 3.3.3 and 3.3.4, respectively. These beamforming techniques can also be applied to SS-based CR systems to control the level of interference at the PUs while enhancing the performance of the SUs. 
The cooperative CR beamforming and the CR beamforming problems are considered in Chapters 6 and 7, respectively.

\subsection{Summary}

This chapter has provided an overview of the CR concept. CR is a technology for improving the wireless spectrum utilisation which is achieved by reusing unused spectrum in a dynamic and opportunistic manner. A CR system is an intelligent wireless communication system that is aware of its surrounding environment and adapts its internal states to statistical variations in the incoming RF stimuli by making corresponding changes in certain operating parameters. Hence, a CR system is able to co-exist with a primary system without degrading the performance of the primary system.

The OSA (interweave) and SS (underlay) are the two basic spectrum access methods for CRs. In the OSA model, the SUs are allowed to transmit in the band of interest when none of the PUs are transmitting in that band. This is achieved through spectrum sensing where the SUs detect active PU signals in the band of interest. A large number of spectrum sensing methods have been proposed in the literature, some of which include energy detection, matched filter detection, second-order statistics and cyclostationarity detection and blind detection. The SS model allows the SUs to transmit concurrently with the PUs in the same band of interest, provided that the SUs are able to limit performance degradation at the PU receivers within some acceptable level. Transmit power control and beamforming are two methods of managing interference in CR underlay systems.

The IEEE has recently ratified the 802.22 WRAN standard [94]. This is the first worldwide standard aimed at utilising the white spaces in the TV spectrum for broad-band access in rural areas using CR technology. 


\section{Chapter 5}

\section{Power Control in Underlay Cognitive Radio Systems with Feasibility Detection}

This chapter considers an underlay CR system with $N$ SU pairs ${ }^{1}$ sharing spectrum with a pair of PUs. The SU power allocation problem is formulated as a sum rate maximisation problem under PU and SU QoS and SU peak power constraints. It is shown that the formulated problem is a GP and can be solved with convex optimisation techniques. The effect of PU transmissions are examined in the formulations. Solutions for both low- and high- SINR scenarios are provided. It is shown that including the PU rate in the optimisation problem in some circumstances leads to increased PU performance while not significantly degrading SU sum rate.

In a practical wireless communications system, accurate CSI is not often available hence power allocation problems are formulated with both perfect and partial CSI and the performance loss incurred due to partial CSI is analysed. Furthermore, a novel method of detecting and removing infeasible SU QoS constraints from the $\mathrm{SU}$ power allocation problem that results in considerably improved SU performance is presented.

Results in the form of rate CDFs for various Rayleigh fading channels are presented.

\subsection{Introduction}

In an underlay $\mathrm{CR}$ system the SUs protect the PU by regulating their transmit power to maintain the PU receiver interference below a well defined threshold level. The limits on this received interference level at the PU receiver can be imposed by an average/peak constraint [85], or a minimum value for its SINR [65].

\footnotetext{
${ }^{1} \mathrm{~A}$ pair consists of a transmitter and a receiver
} 
While imposing an additional CSI requirement [225], the advantage of using an SINR-based PU protection mechanism is that it removes the constant interference threshold, thus benefiting the SUs when the PU link has large SINR.

Power control in CR systems presents unique challenges. In spectrum sharing applications, SU's power must be allocated in a manner that achieves the goals of the CR system while not adversely affecting the operation of the PU. Generally the goals of the $\mathrm{CR}$ are not compatible with the goals of the PU; for instance, increasing SU's power to increase SU's rate will tend to increase interference to the PU. There is a growing body of literature on power control and capacity of CR systems. In [219], soft sensing information was used for optimal power control to maximise capacity of one SU pair coexisting with one PU pair. The impacts of SU's transmission power on the occurrence of spectrum opportunities and the reliability of opportunity detection was analysed in [188]. In [45], dynamic programming was used to develop a power control strategy for one SU pair under a Markov model of the PU's spectrum usage. Optimal power allocation strategies to achieve the ergodic capacity and the outage capacity of one SU pair coexisting with one PU pair under different types of power constraints and fading channel models were obtained in [118]. Power control using game-theoretic approaches has been proposed in $[2,203]$. Power control for CR systems using geometric programming has been proposed in $[115,135,231]$. In [135], a CR relay system with one cognitive source, one relay and a cognitive destination coexisting with a PU pair was considered and an optimisation problem to minimise the total CR transmit power under a peak interference constraint was formulated and solved using geometric programming. A minimax approach was used in [231] to minimise the maximum transmit power for a CR system coexisting with a $P U_{R x}$. The interference caused by a $P U_{T x}$ to the $\mathrm{SU}_{R x} \mathrm{~S}$ was not considered in the problem formulation of [231]. In [115], a distributed approach was used for power allocation to maximise SU sum capacity under a peak interference constraint, but the approach did not include the interference caused by the $\mathrm{PU}_{\mathrm{Tx}}$ and the problem was only analysed for a high SINR scenario.

The contributions of this chapter are as follows.

- We formulate the SU's power allocation problem as a sum rate maximisation problem under PU and SU QoS and SU peak power constraints. We show that it can be solved using geometric programming and convex optimisation techniques.

- Unlike in $[115,135,231,272]$, where the PU's interference at each $\mathrm{SU}_{\mathrm{Rx}}$ is neglected, we evaluate the effect of the PU's interference by explicitly including it in our formulations. We present solutions for both low and high SINR scenarios.

- Most of the CR literature adopts a SU centric view and, apart from guaran- 
teeing minimum QoS to PU, does not consider the PU-SU system as a whole. We show that considering the system sum rate in the optimisation problem, in some circumstances, results in improved PU's performance without a significant penalty in SU's sum rate. Optimisation strategies for different channel conditions are presented.

- We develop a robust SU power allocation problem under channel uncertainties by considering a PU outage probability constraint. Through numerical simulations we show that significant losses in SU's performance can be expected when perfect CSI is not available.

- We present a novel method of detecting and removing infeasible SU's QoS constraints from the SU power allocation problem that results in considerably improved SU's performance.

Although we only consider flat Rayleigh channels, the framework developed in this chapter can be readily extended to other channel models such as Ricean or Nakagami.

\subsection{System Model}

As shown in Fig. 5.1, we consider an underlay CR system with a single PU and $N$ SU transmitters communicating simultaneously over a common channel to their respective receivers. Independent, point-to-point, flat Rayleigh fading channels are assumed for all links in the network. Let $g_{\mathrm{p}}=\left|h_{\mathrm{p}}\right|^{2}, g_{\mathrm{ss}}^{(i j)}=\left|h_{\mathrm{ss}}^{(i j)}\right|^{2}, g_{\mathrm{ps}}^{(i)}=\left|h_{\mathrm{ps}}^{(i)}\right|^{2}$ and $g_{\mathrm{sp}}^{(j)}=\left|h_{\mathrm{sp}}^{(j)}\right|^{2}$ denote the instantaneous channel powers of the $\mathrm{PU}_{\mathrm{Tx}}$ to $\mathrm{PU}_{\mathrm{Rx}}, \mathrm{SU}_{\mathrm{Tx}}$ $j$ to $\mathrm{SU}_{\mathrm{Rx}} i, \mathrm{PU}_{\mathrm{Tx}}$ to $\mathrm{SU}_{\mathrm{Rx}} i$ and $\mathrm{SU}_{\mathrm{Tx}} j$ to $\mathrm{PU}_{\mathrm{Rx}}$ links, respectively. For notational convenience we will denote $g_{\mathrm{s}}^{(i)}=g_{s s}^{(i i)}$. Furthermore, we assume that the channel powers for the PU and each of the $N$ SUs are independent exponentially distributed random variables and are governed by their corresponding parameters $\mathbb{E}\left(g_{p}\right)=\Omega_{\mathrm{p}}$, $\mathbb{E}\left(g_{\mathrm{s}}^{(i)}\right)=\Omega_{\mathrm{s}} \forall i, \mathbb{E}\left(g_{\mathrm{ss}}^{(i j)}\right)=\Omega_{\mathrm{ss}} \forall i \neq j, \mathbb{E}\left(g_{\mathrm{ps}}^{(i)}\right)=\Omega_{\mathrm{ps}} \forall i$ and $\mathbb{E}\left(g_{\mathrm{sp}}^{(j)}\right)=\Omega_{\mathrm{sp}} \forall j$.

In our model the SINR at the $i$ th $\mathrm{SU}$ receiver is given by

$$
\gamma_{\mathrm{s}}^{(i)}=\frac{P_{\mathrm{s}}^{(i)} g_{\mathrm{s}}^{(i)}}{\sum_{j=1, j \neq i}^{N} P_{\mathrm{s}}^{(j)} g_{\mathrm{ss}}^{(i j)}+P_{\mathrm{p}} g_{\mathrm{ps}}^{(i)}+\sigma_{\mathrm{s}}^{2}}
$$

and that at the PU receiver by

$$
\gamma_{\mathrm{p}}=\frac{P_{\mathrm{p}} g_{\mathrm{p}}}{\sum_{j=1}^{N} P_{\mathrm{s}}^{(j)} g_{\mathrm{sp}}^{(j)}+\sigma_{\mathrm{p}}^{2}}
$$




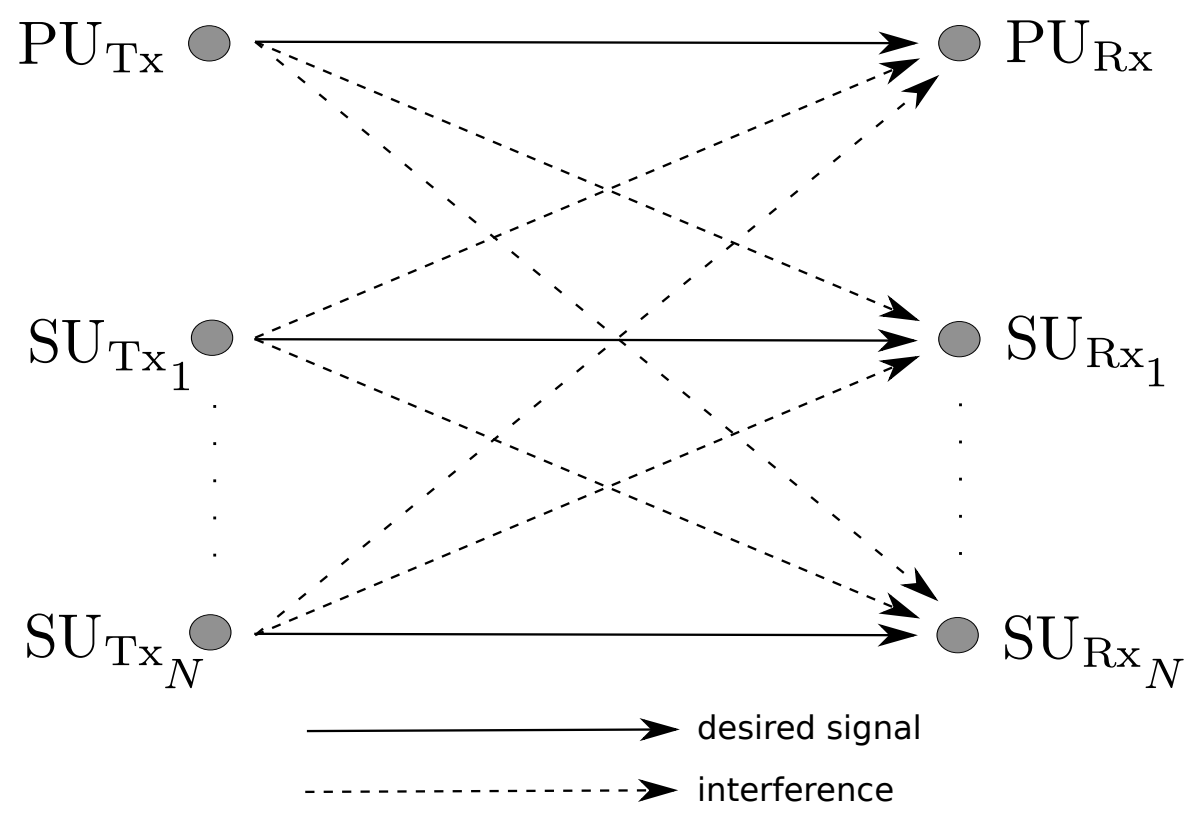

Figure 5.1: System Model

where $P_{\mathrm{s}}^{(j)}$ and $P_{\mathrm{p}}$ are the $j$ th SU and PU transmit powers, respectively, and $\sigma_{\mathrm{s}}^{2}$ and $\sigma_{\mathrm{p}}^{2}$ are the AWGN variance at the $i$ th $\mathrm{SU}_{\mathrm{Rx}}$ and $\mathrm{PU}_{\mathrm{Rx}}$, respectively. We also note that that there is a maximum transmit power constraint, $P_{\mathrm{s}, \max }^{(j)}$, on the $\mathrm{SU}$ transmitters which may be due either to regulatory or hardware limitations. This is denoted by

$$
P_{\mathrm{s}}^{(j)} \leq P_{\mathrm{s}, \max }^{(j)}
$$

Additionally, the vector $\mathbf{P}_{\mathrm{s}}$ is used to collectively refer to the set of SU transmit powers, i.e., $\mathbf{P}_{\mathrm{s}} \triangleq\left[P_{\mathrm{s}}^{(1)} \ldots P_{\mathrm{s}}^{(N)}\right]^{T}$.

In an underlay CR system the secondary users are allowed to operate as long as they can guarantee a certain level of QoS to the primary user. Hence, in our analysis we impose an SINR constraint, $\gamma_{T}$, at the PU receiver, $\gamma_{\mathrm{p}} \geq \gamma_{\mathrm{T}}$.

The PU's rate is given by

$$
R_{\mathrm{p}}=\log _{2}\left(1+\gamma_{\mathrm{p}}\right)
$$

while the SU's sum rate is denoted by

$$
R_{\Sigma}=\sum_{i=1}^{N} R_{i}
$$

where the individual rate of the $i$ th $\mathrm{SU}$ is given by

$$
R_{i}=\log _{2}\left(1+\gamma_{\mathrm{s}}^{(i)}\right)
$$


Using (5.3) and (5.4), the system sum rate can then be expressed as

$$
R_{\mathrm{sys}}=R_{\mathrm{p}}+R_{\Sigma}
$$

$R_{\Sigma}$ and $R_{\text {sys }}$ are the performance metrics optimised in Sections 5.3-5.6 of this chapter.

The main system variables can be parameterised as follows. We denote by $c_{1}=\Omega_{\mathrm{sp}} / \Omega_{\mathrm{s}}$ the ratio of interference to desired channel power. Similarly,

$$
c_{2}=\frac{\gamma_{\mathrm{T}}}{P_{\mathrm{p}} \Omega_{\mathrm{p}} / \sigma_{\mathrm{p}}^{2}}
$$

represents the ratio of the minimum target SINR to the mean SNR at the $\mathrm{PU}_{\mathrm{Rx}}$. Hence, increasing $c_{2}$ corresponds to reducing the allowable interference, with the case of $c_{2}=1$ corresponding to zero average allowable interference. Finally, $c_{3}=$ $\Omega_{\mathrm{sS}} / \Omega_{\mathrm{S}}$ is the ratio of desired channel power to interfering $\mathrm{SU}$ link channel power.

\subsection{SU Power Optimisation}

In this section, we aim to find the SU's power allocation such that the SU's sum rate, $R_{\Sigma}$, or the system sum rate, $R_{\text {sys }}$, is maximised while maintaining the PU receiver QoS above the threshold $\gamma_{\mathrm{T}}$, and keeping within the SU's transmit power budget. We may additionally choose to set minimum SINR thresholds, $\gamma_{\mathrm{s}, \min }^{(i)}$ on the $i$ th SU receiver. This represents a practical limitation on SU receivers below which the receivers fail to operate with acceptable performance. We assume that the power allocation problem is solved by a central SU controller and a control channel for the exchange of all necessary information needed for solving the problem exists. Furthermore, we assume that we are unable to control the PU's transmit power and the PU transmits at a constant power of $P_{\mathrm{p}}$. In this section, we formulate the $\mathrm{SU}$ power allocation problem under the assumption that perfect CSI for all links are available which allows us to obtain fundamental limits on rate. However, in practice the channel gains would need to be estimated, hence the capacities obtained in this section provide an upper bound. In Section 5.5, we consider the case when perfect CSI is not available and there is a non-zero probability of PU outage which we constrain. Mathematically we solve the following suite of optimisation problems.

1. SU Sum Rate Maximisation:

$$
\begin{aligned}
\max _{\mathbf{P}_{\mathrm{s}}} & R_{\Sigma} \\
\text { s.t. } & \gamma_{\mathrm{p}} \geq \gamma_{\mathrm{T}}, \\
& P_{\mathrm{s}}^{(j)} \leq P_{\mathrm{s}, \max }^{(j)}, \quad j=1, \ldots, N \\
\text { (and o.s.t.) } & \gamma_{\mathrm{s}}^{(i)} \geq \gamma_{\mathrm{s}, \min }^{(i)}, \quad i=1, \ldots, N
\end{aligned}
$$


where s.t. and o.s.t. stand for "subject to" and "optionally subject to", respectively. The only difference between problems (5.8a)-(5.8c) and (5.8a)-(5.8d) is that (5.8a)-(5.8d) includes SU QoS constraints, whereas (5.8a)-(5.8c) does not.

2. System Sum Rate Maximisation:

$$
\begin{aligned}
\max _{\mathbf{P}_{\mathrm{s}}} & R_{\mathrm{sys}} \\
\text { s.t. } & (5.8 \mathrm{~b}) \text { and }(5.8 \mathrm{c}), \\
\text { (and o.s.t.) } & (5.8 \mathrm{~d}) .
\end{aligned}
$$

From (5.4) and (5.5) it is obvious that maximising the objective in (5.8) is equivalent to maximising $\prod_{i=1}^{N}\left(1+\gamma_{\mathrm{s}}^{(i)}\right)$. Similarly, for (5.9) we seek to maximise $\left(1+\gamma_{\mathrm{p}}\right) \cdot \prod_{i=1}^{N}\left(1+\gamma_{\mathrm{s}}^{(i)}\right)$. Problems (5.8) and (5.9) can be modified to minimisation problems by taking the reciprocal of the objectives. The suite of optimisation problems are nonlinear and non-convex and generally hard to solve [29]. We proceed by dividing our problem into high and low SINR scenarios.

\subsubsection{High SINR Scenario}

When the SINR at every receiver is high, $R_{\mathrm{p}}, R_{\Sigma}$ and $R_{\text {sys }}$ given in (5.3)-(5.6) can be approximated by

$$
\begin{aligned}
& R_{\mathrm{p}} \approx \log _{2}\left(\gamma_{\mathrm{p}}\right) \\
& R_{\Sigma} \approx \log _{2}\left(\prod_{i=1}^{N} \gamma_{\mathrm{s}}^{(i)}\right) \\
& R_{\mathrm{sys}} \approx \log _{2}\left(\gamma_{\mathrm{p}} \cdot \prod_{i=1}^{N} \gamma_{\mathrm{s}}^{(i)}\right) .
\end{aligned}
$$

These approximations are accurate when $\gamma_{\mathrm{p}}$ and $\gamma_{\mathrm{s}}^{(i)}$ are much larger than $0 \mathrm{~dB}$, e.g., $10 \mathrm{~dB}$ or more. Using the approximations in (5.10), the optimisation problems (5.8) and (5.9) can be written in minimisation form as

1. High SINR SU Sum Rate Maximisation :

$$
\begin{array}{ll}
\min _{\mathbf{P}_{\mathrm{s}}} & \prod_{i=1}^{N}\left(\frac{1}{\gamma_{\mathrm{s}}^{(i)}}\right) \\
\text { s.t. } & (5.8 \mathrm{~b}),(5.8 \mathrm{c}) \text { and optionally }(5.8 \mathrm{~d}) .
\end{array}
$$


2. High SINR System Sum Rate Maximisation :

$$
\begin{array}{ll}
\min _{\mathbf{P}_{\mathrm{s}}} & \left(\frac{1}{\gamma_{\mathrm{p}}}\right) \cdot \prod_{i=1}^{N}\left(\frac{1}{\gamma_{\mathrm{s}}^{(i)}}\right) \\
\text { s.t. } & (5.8 \mathrm{~b}),(5.8 \mathrm{c}) \text { and optionally }(5.8 \mathrm{~d}) .
\end{array}
$$

Problems (5.11) and (5.12) are GPs and can be transformed to convex optimisation problems and solved efficiently in polynomial time by interior point methods [175].

Through straightforward manipulation of the second and third constraints, problems (5.11) and (5.12) can be transformed into a standard form GP [29]. Once in this form, they can be solved to obtain the optimum SU power allocation. The resulting performance is evaluated in Section 5.7.

\subsubsection{Low SINR Scenario}

In the low SINR scenario the approximation (5.10) is no longer valid and so our sum rate maximisation optimisation problems are given by

1. Low SINR SU Sum Rate Maximisation :

$$
\begin{array}{ll}
\min _{\mathbf{P}_{\mathrm{s}}} & \prod_{i=1}^{N}\left(\frac{1}{1+\gamma_{\mathrm{s}}^{(i)}}\right) \\
\text { s.t. } & (5.8 \mathrm{~b}),(5.8 \mathrm{c}) \text { and optionally (5.8d). }
\end{array}
$$

2. Low SINR System Sum Rate Maximisation :

$$
\begin{array}{ll}
\min _{\mathbf{P}_{\mathrm{s}}} & \left(\frac{1}{1+\gamma_{\mathrm{p}}}\right) \cdot \prod_{i=1}^{N}\left(\frac{1}{1+\gamma_{\mathrm{s}}^{(i)}}\right) \\
\text { s.t. } & (5.8 \mathrm{~b}),(5.8 \mathrm{c}) \text { and optionally }(5.8 \mathrm{~d}) .
\end{array}
$$

The objectives in problems (5.13) and (5.14) are ratios of posynomials and hence they are not themselves posynomials. Optimisation problems of this nature are not GP and are known as Complementary GP [18]. Complementary GPs are non-convex problems but can be solved with an iterative technique known as the single condensation method [18]. In each iteration, the feasible point computed in the previous iteration is used to approximate the denominator of the objective monomial. Since a ratio of posynomial and monomial is a posynomial [29], the resulting problem is a GP. The procedure is repeated until the solution converges on an optimum of the original Complementary GP. It should be noted that convergence to a local or global minimum is possible; however, extensive numerical experiments (Section 5.7) have found empirically that the solution practically always converges 
to the global minimum ${ }^{2}$. The posynomial is approximated with a monomial using the geometric-arithmetic mean inequality [18]

$$
\sum_{k} \delta_{k} v_{k} \geq \prod_{k} v_{k}^{\delta_{k}}
$$

where $v_{k} \geq 0, \delta_{k} \geq 0$ and $\sum_{k} \delta_{k}=1$. If we let $u_{k}=\delta_{k} v_{k}$, then (5.15) can be written as

$$
\sum_{k} u_{k} \geq \prod_{k}\left(\frac{u_{k}}{\delta_{k}}\right)^{\delta_{k}}
$$

Note that equality in (5.16) holds when $\delta_{k}=u_{k} / \sum_{k} u_{k}$. The term on the left hand side of (5.16) resembles the denominator of our objective, i.e., a sum of monomials. Hence, if we let $u_{k}\left(\mathbf{P}_{\mathrm{s}}\right)$ be the monomial terms of the denominator and $\delta_{k}=u_{k}\left(\mathbf{P}_{\mathrm{s}}\right) / \sum_{k} u_{k}\left(\mathbf{P}_{\mathrm{s}}\right)$, then from (5.16) it is clear that the denominator can be approximated around a feasible $\mathbf{P}_{\mathrm{s}}$ with a product of monomials. Since the approximation is always an under-estimator of the original posynomial, minimising the condensed objective guarantees that the solution moves towards a minimum of the original objective function. An adaptation of a commonly used algorithm [18, 46] for solving the low SINR sum rate maximisation problem is presented below.

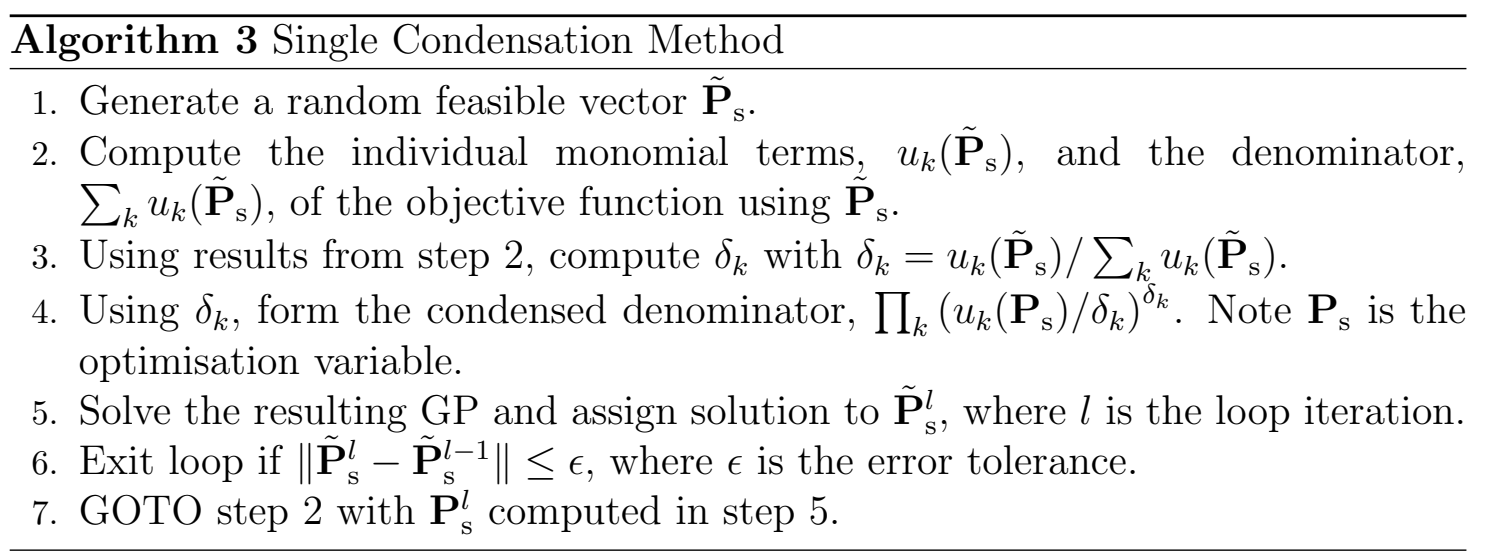

The single condensation method presented above is a general method of solving the power allocation problem and can also be used to solve the high SINR scenario without using the approximation (5.10) or mixed scenario cases in which some of the receivers in the system have high SINR and others have low SINR.

\footnotetext{
${ }^{2}$ Each instantiation of the problem is solved multiple times using different random but feasible starting points and solutions checked to confirm convergence to the same point. If different solutions are obtained then this indicates convergence to local minima, but in our extensive numerical experiments we have never observed this behaviour. This suggests that the solution very likely converges to the global minimum.
} 


\subsection{SU Power Optimisation with Feasibility De- tection}

Optimisation problems (5.8a)-(5.8d) and (5.9a)-(5.9c) are infeasible if any one of the SU QoS constraints is infeasible. This has an adverse effect on the sum rate since no SUs are able to access the channel if the QoS constraint cannot be met for any one (or more) SU. Thus, one SU which violates the QoS constraint renders the entire optimisation infeasible. The sum rate can be improved by selecting the optimum subset of SU's that do not violate the QoS constraints and maximise the sum rate. However, this selection process is a NP-hard combinatorial optimisation problem which is extremely difficult to solve. A suboptimal method that improves the sum rate is to exclude the violating SUs from transmission. In this section we formulate a method of detecting and removing the violating SUs from the optimisation problem. The issue of fairness among SUs and the tradeoff between fairness and rate is beyond the scope of this work. Our method is based on the sum of infeasibilities feasibility detection technique introduced in Section 2.8.6 on page 31 . We form the problem

$$
\begin{array}{ll}
\min _{\mathbf{P}_{\mathrm{s}}, \mathbf{s}} & \mathbf{1}^{\mathrm{T}} \mathbf{s} \\
\text { s.t. } & \frac{\gamma_{\mathrm{T}}}{\gamma_{\mathrm{p}}} \leq \mathbf{s}_{0}, \\
& \frac{\gamma_{\mathrm{s}, \min }^{(i)} \leq \mathbf{s}_{i}, \quad i=1, \ldots, N}{\gamma_{\mathrm{s}}^{(i)}} \\
& P_{\mathrm{s}}^{(j)} \leq P_{\mathrm{s}, \max }^{(j)}, \quad j=1, \ldots, N \\
& \mathbf{s} \succeq \mathbf{1},
\end{array}
$$

where $\mathbf{1}$ is a vector of length $N+1$ with all entries equal to one and $\mathbf{s} \in \mathbb{R}_{++}^{N+1}$. For fixed $\mathbf{P}_{\mathrm{s}}$, the optimal values of $\mathbf{s}_{0}$ and $\mathbf{s}_{i}$ are $\max \left(\gamma_{\mathrm{T}} / \gamma_{\mathrm{p}}, 1\right)$ and $\max \left(\gamma_{\mathrm{s}, \min }^{(i)} / \gamma_{\mathrm{s}}^{(i)}, 1\right)$ respectively, so in problem (5.17), we are minimising the sum of the infeasibilities. The optimum value of (5.17) is $N+1$ and achieved if and only if the constraints (5.8b), (5.8c) and (5.8d) are feasible. It follows that all feasible SU QoS constraints will have the corresponding element in the vector $\mathbf{s}$ equal to one.

Let $\mathcal{I}$ represent the set of feasible SU QoS constraints determined from the solution of problem (5.17). We then solve the following SU power optimisation problems in which the violating SUs are removed

1. SU Sum Rate Maximisation with Feasible SU QoS Constraints:

$$
\begin{array}{ll}
\max _{\mathbf{P}_{\mathrm{s}}} & R_{\Sigma} \\
\text { s.t. } & \gamma_{\mathrm{p}} \geq \gamma_{\mathrm{T}}, \\
& \gamma_{\mathrm{s}}^{(i)} \geq \gamma_{\mathrm{s}, \min }^{(i)}, \quad i \in \mathcal{I}
\end{array}
$$




$$
P_{\mathrm{s}}^{(j)} \leq P_{\mathrm{s}, \max }^{(j)}, \quad j \in \mathcal{I}
$$

2. System Sum Rate Maximisation with Feasible SU QoS Constraints:

$$
\begin{array}{ll}
\max _{\mathbf{P}_{\mathrm{s}}} & R_{\mathrm{sys}} \\
\text { s.t. } & (5.18 \mathrm{~b}),(5.18 \mathrm{c}) \text { and }(5.18 \mathrm{~d}) .
\end{array}
$$

Problems (5.18) and (5.19) can be solved using methods presented in Section 5.3 for the low and high SINR scenarios.

\subsection{SU Power Optimisation Under Channel Un- certainties}

So far we have assumed that perfect CSI for all links is available. However, in practise this assumption may not be valid. For our analysis, we assume that the channel between the $\mathrm{SU}_{\mathrm{Tx}} \mathrm{S}$ and $\mathrm{SU}_{\mathrm{Rx}} \mathrm{S}$ are accurately known through the SU's channel estimation procedure and those between the PU transmitter and SU receivers can be accurately measured, for example through knowledge of the PU's pilot symbols. As stated in Section 5.2, the $\mathrm{PU}_{\mathrm{Tx}}$ to $\mathrm{PU}_{\mathrm{Rx}}$ and the $j$ th $\mathrm{SU}_{\mathrm{Tx}}$ to $\mathrm{PU}_{\mathrm{Rx}}$ channel gains are iid and for the analysis of this section, we assume that only the mean channel gains, $\Omega_{\mathrm{p}}$ and $\Omega_{\mathrm{sp}}$, of these links are known (only partial CSI is available), i.e., the instantaneous values of $g_{\mathrm{p}}$ and $g_{\mathrm{sp}}^{(j)}$ are not known. In this section we consider the SU power optimisation problem under these uncertainties.

We build on the approach taken in [117] (see Section 3.3.1) and consider the PU's outage probability as a QoS parameter. In the system under consideration, outage occurs when the PU's SINR, $\gamma_{\mathrm{p}}$, falls below the PU's SINR threshold, $\gamma_{\mathrm{T}}$. The outage probability is expressed as

$$
\begin{aligned}
\mathrm{P}_{\mathrm{o}} & =\operatorname{Prob}\left(\gamma_{\mathrm{p}} \leq \gamma_{\mathrm{T}}\right) \\
& =\operatorname{Prob}\left(P_{\mathrm{p}} g_{\mathrm{p}} \leq \gamma_{\mathrm{T}}\left(\sum_{j=1}^{N} P_{\mathrm{s}}^{(j)} g_{\mathrm{sp}}^{(j)}+\sigma_{\mathrm{p}}^{2}\right)\right)
\end{aligned}
$$

In a Rayleigh fading environment, $g_{\mathrm{p}}$ and $g_{\mathrm{sp}}^{(j)}$ are exponentially distributed random variables with means $\Omega_{\mathrm{p}}$ and $\Omega_{\mathrm{sp}}$ respectively. Under these conditions, the outage probability is known to have the following form [117, 222, 251, 252]

$$
\mathrm{P}_{\mathrm{o}}=1-e^{-c_{2}} \prod_{j=1}^{N}\left(\frac{1}{1+\frac{\gamma_{\mathrm{T}} P_{\mathrm{s}}^{(j)} \Omega_{\mathrm{sp}}}{P_{\mathrm{p}} \Omega_{\mathrm{p}}}}\right)
$$

where $c_{2}$ is given by (5.7). 
To formulate the SU power optimisation problem under channel uncertainty, we replace the PU's SINR threshold in problems (5.8) and (5.9) by the outage probability constraint. Furthermore, since instantaneous CSI for $\mathrm{PU}_{\mathrm{Tx}}$ to $\mathrm{PU}_{\mathrm{Rx}}$ and $\mathrm{SU}_{\mathrm{Tx}} \mathrm{S}$ to $\mathrm{PU}_{\mathrm{Rx}}$ links are not available, in the optimisation problem that seeks to maximise the system sum rate - (5.9) - the PU's SINR is calculated using the expected values of these links i.e., $g_{p}$ and $g_{\mathrm{sp}}^{(j)}$ in (5.2) are replaced with $\Omega_{\mathrm{p}}$ and $\Omega_{\text {sp }}$ respectively. Hence, given a maximum allowable outage probability, $\mathrm{P}_{\mathrm{o}, \max }$, the optimisation problems are expressed as:

1. SU Sum Rate Maximisation Under Channel Uncertainty:

$$
\begin{aligned}
\max _{\mathbf{P}_{\mathrm{s}}} & R_{\Sigma} \\
\text { s.t. } & \prod_{j=1}^{N}\left(1+\frac{\gamma_{\mathrm{T}} P_{\mathrm{s}}^{(j)} \Omega_{\mathrm{sp}}}{P_{\mathrm{p}} \Omega_{\mathrm{p}}}\right) \leq \frac{e^{-c_{2}}}{1-\mathrm{P}_{\mathrm{o}, \max }}, \\
& (5.8 \mathrm{c}), \\
\text { (and o.s.t.) } & (5.8 \mathrm{~d}) .
\end{aligned}
$$

2. System Sum Rate Maximisation Under Channel Uncertainty:

$$
\begin{aligned}
\max _{\mathbf{P}_{\mathrm{s}}} & R_{\mathrm{sys}} \\
\text { s.t. } & (5.8 \mathrm{c}) \text { and }(5.21 \mathrm{~b}), \\
\text { (and o.s.t.) } & (5.8 \mathrm{~d}) .
\end{aligned}
$$

We have only shown the general formulations here. However, using the approximations in Section 5.3.1, sum rate maximisation problems for the high SINR scenario can be constructed. Problems (5.21) and (5.22) can be solved using techniques described in Sections 5.3.1 and 5.3.2.

\subsection{SU Power Optimisation With Feasibility De- tection Under Channel Uncertainties}

The feasibility detection technique formulated in Section 5.4 can be incorporated into the formulations developed in Section 5.5 to improve the SU's performance when SU's QoS constraints are imposed. We form the problem

$$
\begin{array}{ll}
\min _{\mathbf{P}_{\mathrm{s}, \mathbf{s}}} & \mathbf{1}^{\mathrm{T}} \mathbf{S} \\
\text { s.t. } & \left(\frac{1-\mathrm{P}_{\mathrm{o}, \max }}{e^{-c_{2}}}\right) \prod_{j=1}^{N}\left(1+\frac{\gamma_{\mathrm{T}} P_{\mathrm{s}}^{(j)} \Omega_{\mathrm{sp}}}{P_{\mathrm{p}} \Omega_{\mathrm{p}}}\right) \leq \mathbf{s}_{0},
\end{array}
$$




$$
\begin{array}{ll}
\frac{\gamma_{\mathrm{s}, \text { min }}^{(i)}}{\gamma_{\mathrm{s}}^{(i)}} \leq \mathbf{s}_{i}, & i=1, \ldots, N \\
P_{\mathrm{s}}^{(j)} \leq P_{\mathrm{s}, \max }^{(j)}, & j=1, \ldots, N \\
\mathbf{s} \succeq \mathbf{1}, &
\end{array}
$$

the solution of which gives us the set $\mathcal{I}$, the set of feasible SU QoS constraints. As in Section 5.4, we then solve the following problems in which the violating SUs are removed

1. SU Sum Rate Maximisation Under Channel Uncertainty with Feasible SU QoS Constraints:

$$
\begin{array}{ll}
\max _{\mathbf{P}_{\mathrm{s}}} & R_{\Sigma} \\
\text { s.t. } & (5.21 \mathrm{~b}), \\
& \gamma_{\mathrm{s}}^{(i)} \geq \gamma_{\mathrm{s}, \text { min }}^{(i)}, \quad i \in \mathcal{I} \\
& P_{\mathrm{s}}^{(j)} \leq P_{\mathrm{s}, \text { max }}^{(j)}, \quad j \in \mathcal{I}
\end{array}
$$

2. System Sum Rate Maximisation Under Channel Uncertainty with Feasible SU QoS Constraints:

$$
\begin{array}{ll}
\max _{\mathbf{P}_{\mathrm{s}}} & R_{\mathrm{sys}} \\
\text { s.t. } & (5.21 \mathrm{~b}),(5.24 \mathrm{c}) \text { and }(5.24 \mathrm{~d}) .
\end{array}
$$

Problems (5.24) and (5.25) can be solved using the methods presented in Section 5.3 for the low and high SINR scenarios.

\subsection{Simulation Results and Discussion}

We now present the results of simulations that require solution of the optimisation problems formulated in this chapter, specifically evaluating the CDFs of the resulting capacities. We consider a system with $N=3 \mathrm{SUs}$. In all simulations we have set $P_{\mathrm{p}}=30 \mathrm{dBm}, P_{\mathrm{s}, \max }^{(i)}=30 \mathrm{dBm}, \sigma_{\mathrm{p}}^{2}=\sigma_{\mathrm{s}}^{2}=-7 \mathrm{dBm}$ and $\Omega_{\mathrm{p}}=\Omega_{\mathrm{s}}=5 \mathrm{~dB}$. This choice of parameter values allows us to contrast the optimisation problems formulated in this chapter in the channel scenarios given below. Simulations for optimisation problems that impose SU QoS requirements have $\gamma_{\mathrm{s}, \text { min }}^{(i)}=-10 \mathrm{~dB}$, $i=1, \ldots, N$. In SU power optimisation problems under channel uncertainties we have set the outage probability, $\mathrm{P}_{\mathrm{o} \text {,max }}$, to $5 \%$. We consider the following three channel scenarios

1. Scenario A: High Interference

In this scenario $c_{1}=c_{3}=0.9$ which corresponds to each receiver being 
approximately the same distance from all transmitters. This results in high interference among all users, thus making the PU's QoS constraint difficult to satisfy. The SINR is expected to be low, hence we use the low SINR method of Section 5.3.2 to obtain the solution.

\section{Scenario B: Low PU and High SU Interference}

In this scenario $c_{1}=0.1$ and $c_{3}=0.9$. Here, the PU experiences low interference from the SUs since it is approximately 3 times (assuming $1 / d^{2}$ path loss) further away from $\mathrm{SU}_{\mathrm{Tx}} \mathrm{s}$ than the $\mathrm{PU}_{\mathrm{Tx}}$. As a result, the PU's QoS constraint is easily satisfied. However, SU to SU interference is very prominent. In this scenario, the SINR at the SUs will be low and therefore we obtain the solution using the low SINR method of Section 5.3.2.

\section{Scenario $C$ : Low Interference}

In this scenario $c_{1}=c_{3}=0.1$ which corresponds to each receiver being approximately 3 times further away from the interfering transmitters than its own transmitter. This results in low interference between all users, thus making the PU's QoS constraint easy to satisfy. Since the SINR at all receivers is expected to be high, this scenario is solved using the high SINR method of Section 5.3.1.

When solving using the low SINR (single condensation) method, each instantiation of the problem is solved multiple times using different random but feasible starting points, $\tilde{\mathbf{P}}_{\mathrm{s}}$, and solutions checked to confirm convergence to the same point. If different solutions are obtained then this indicates convergence to local minima, but in our extensive numerical experiments we have never observed this behaviour. This suggests that the solution practically always converges to the global minimum.

For our discussion, we define SU blocking probability as the probability that $R_{\Sigma}=0$, i.e., no SUs are able to access the channel.

Results of our proposed methods are compared against the equal power allocation method and a power profile method analogous to the "poor man's waterfilling" method [215] where we allocate power proportionally to $g_{\mathrm{s}}^{(i)} / g_{\mathrm{sp}}^{(i)}$. We refer to these methods as ad hoc allocation methods. Note that the ad hoc allocation methods do not impose a minimum SU QoS requirement, hence a fair comparison is only possible against the SU and system sum rate maximisation problems without SU QoS constraints ((5.8a)-(5.8c) and $(5.9 \mathrm{a}-5.9 \mathrm{~b}))$.

We also compare our proposed feasibility detection method against the brute force method of selecting the optimum set of SU transmitters. The brute force method performs an exhaustive search and finds the set of SUs that achieve the highest sum rate.

Figs. $5.2-5.7$ show $R_{\Sigma}$ and $R_{\mathrm{p}}$ rate CDFs for the three channel conditions with $\gamma_{\mathrm{T}}=5 \mathrm{~dB}$ when perfect CSI is available. The legends of Figs. 5.2 and 5.3 


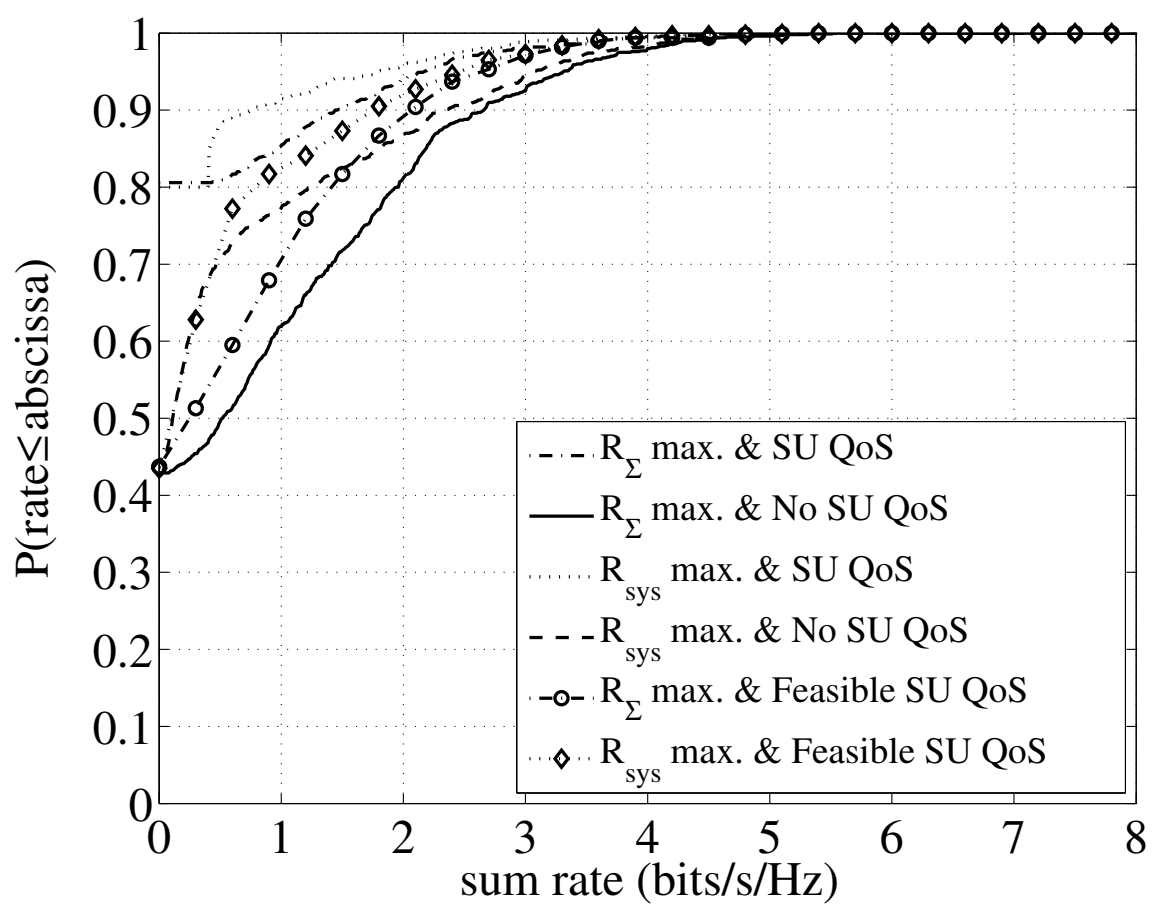

Figure 5.2: Sum rate CDF with perfect CSI for Scenario $A, \gamma_{\mathrm{T}}=5 \mathrm{~dB}$. The same legend is used in Figs. 5.3-5.7.

are applicable for all 6 of these figures. From Figs. 5.2, 5.4 and 5.6, we observe that in all three scenarios, the SU sum rate maximisation problem without SU QoS constraints ((5.8a)-(5.8c)) performs the best in terms of SU performance. All optimisation problems result in a non-zero SU blocking probability due to either PU's QoS constraints or both PU's and SU's QoS constraints. It is evident that feasibility detection results in a significant improvement in SU's performance in all channel scenarios, for instance, in Scenario $A$ the SU blocking probability (Fig. 5.2 ) is reduced from $80 \%$ to approximately $42 \%$. Figs. 5.3, 5.5 and 5.7 show the PU's rate CDF for the three scenarios along with the CDF for the reference case when no SUs are transmitting. The discontinuity in the graphs correspond to the point at which the optimisation problems become feasible and SU transmissions start. The effect of the SU's transmissions on the PU's rate is clearly visible.

By noting the effects of including the PU's rate in the optimisation problems we are able to contrast two optimisation strategies using the $\mathrm{SU}$ and system sum rate maximisation problems without SU QoS constraints ((5.8a)-(5.8c) and (5.9a)(5.9b)). In Scenario A, we observe that the two optimisation problems result in similar median SU sum capacities; however, the system sum rate maximisation problem results in a much improved median PU's rate. Hence, when the PU experiences high interference, the system sum rate maximisation problem is the better option as it results in improved PU's performance while not adversely affecting the SU's performance. On the other hand, in Scenarios $B$ and $C$, where there is low interference to the PU, using the system sum rate maximisation problem improves 


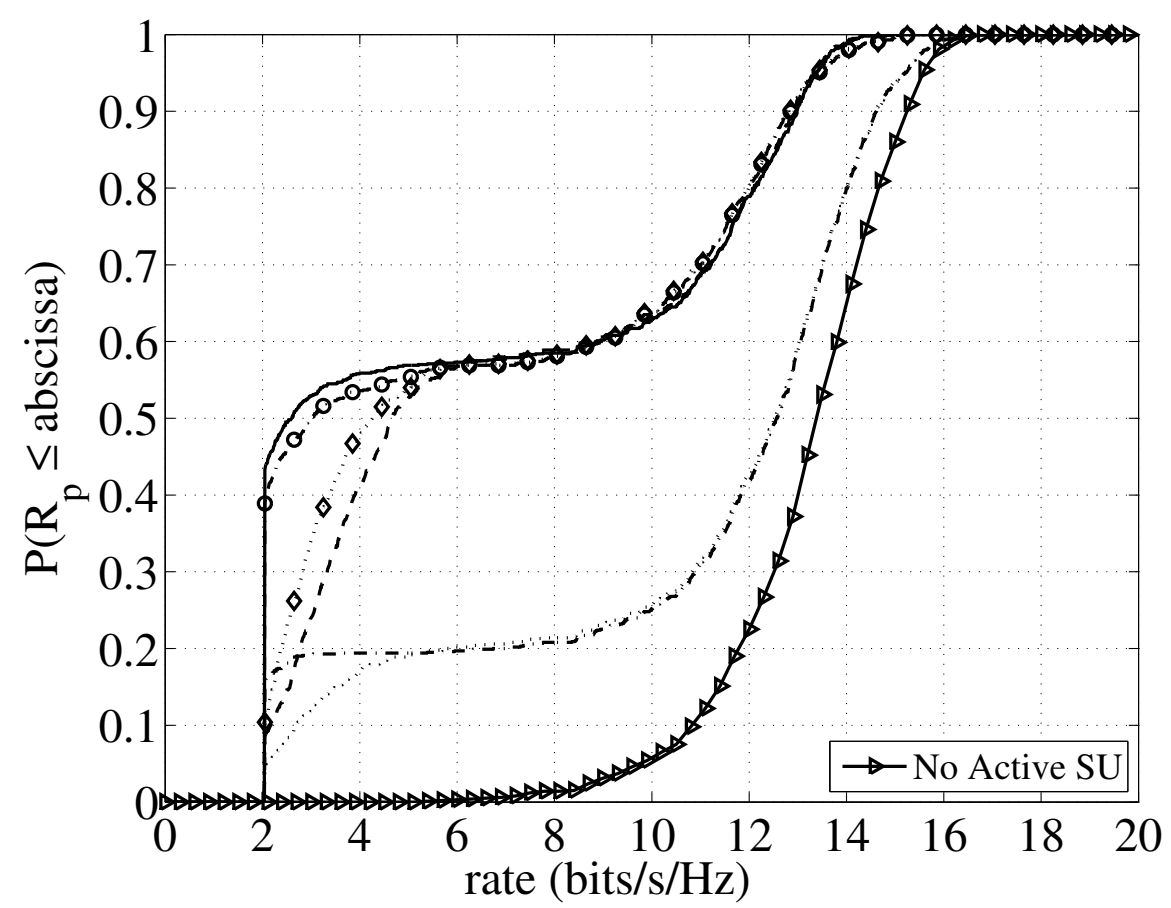

Figure 5.3: $R_{\mathrm{p}}$ CDF with perfect CSI for Scenario $A, \gamma_{\mathrm{T}}=5 \mathrm{~dB}$.

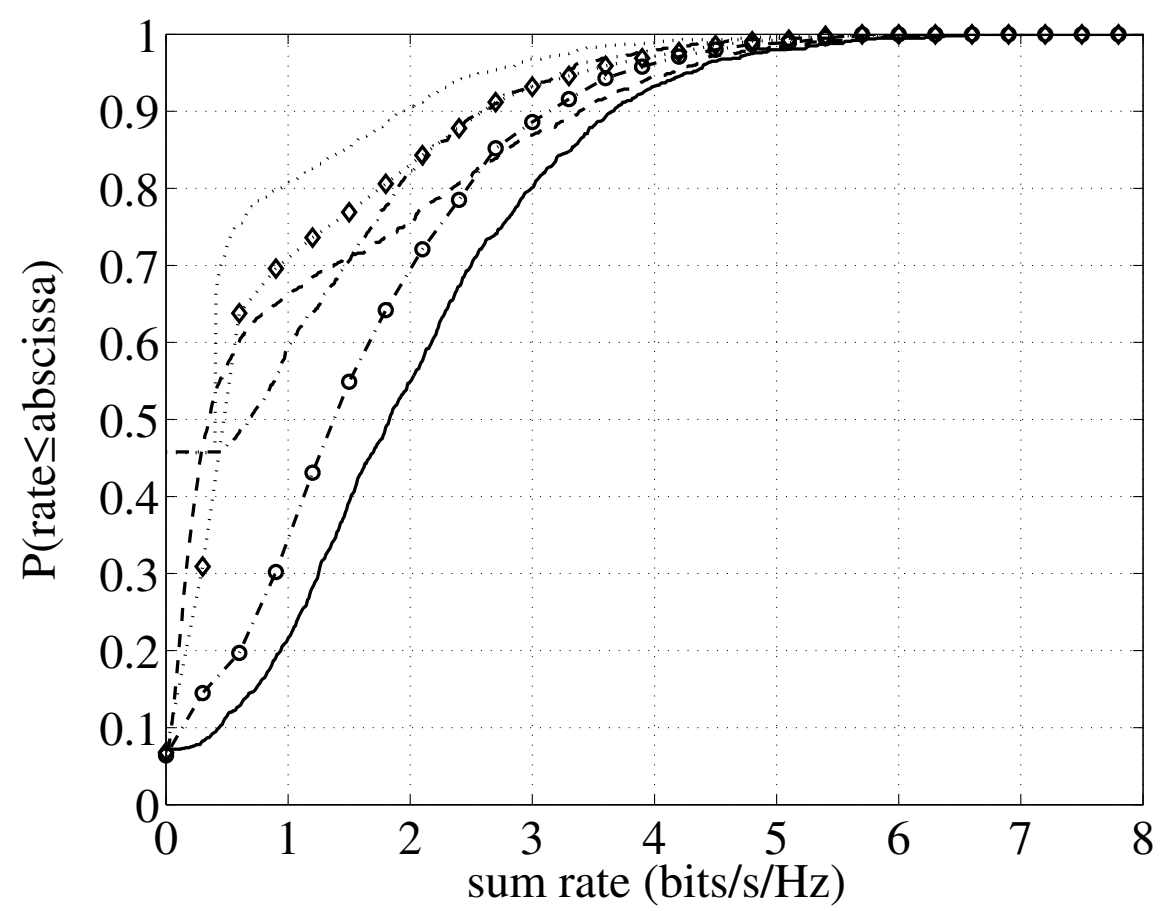

Figure 5.4: Sum rate CDF with perfect CSI for Scenario $B, \gamma_{\mathrm{T}}=5 \mathrm{~dB}$. 


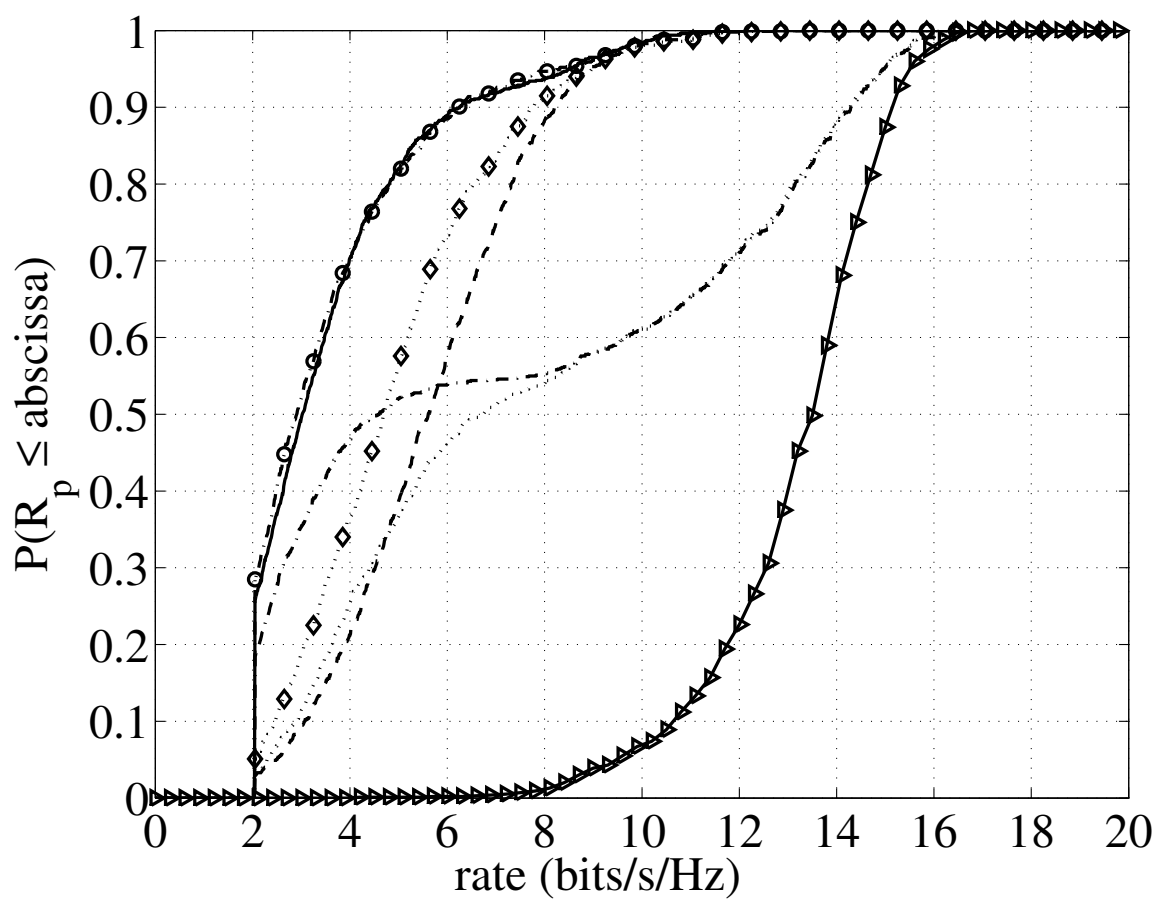

Figure 5.5: $R_{\mathrm{p}}$ CDF with perfect CSI for Scenario $B, \gamma_{\mathrm{T}}=5 \mathrm{~dB}$.

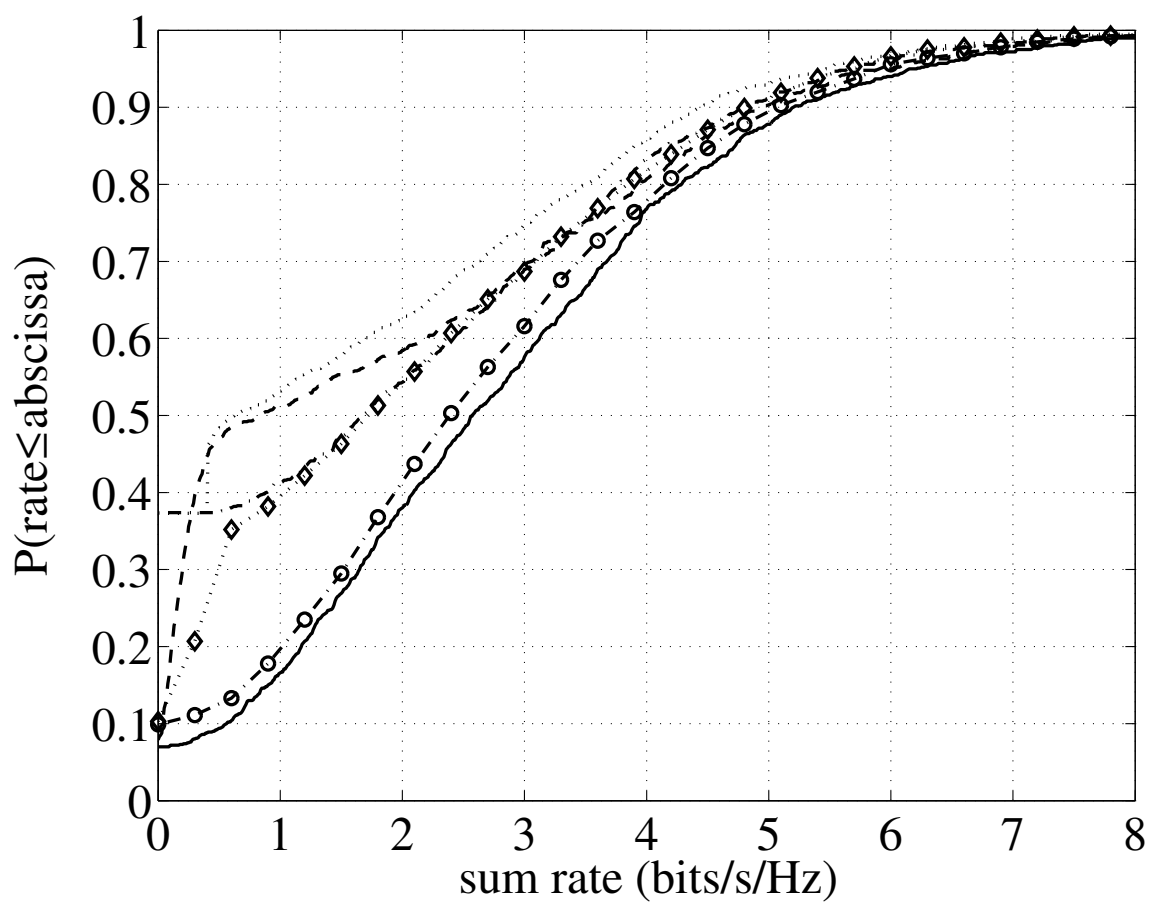

Figure 5.6: Sum rate CDF with perfect CSI for Scenario $C, \gamma_{\mathrm{T}}=5 \mathrm{~dB}$. 


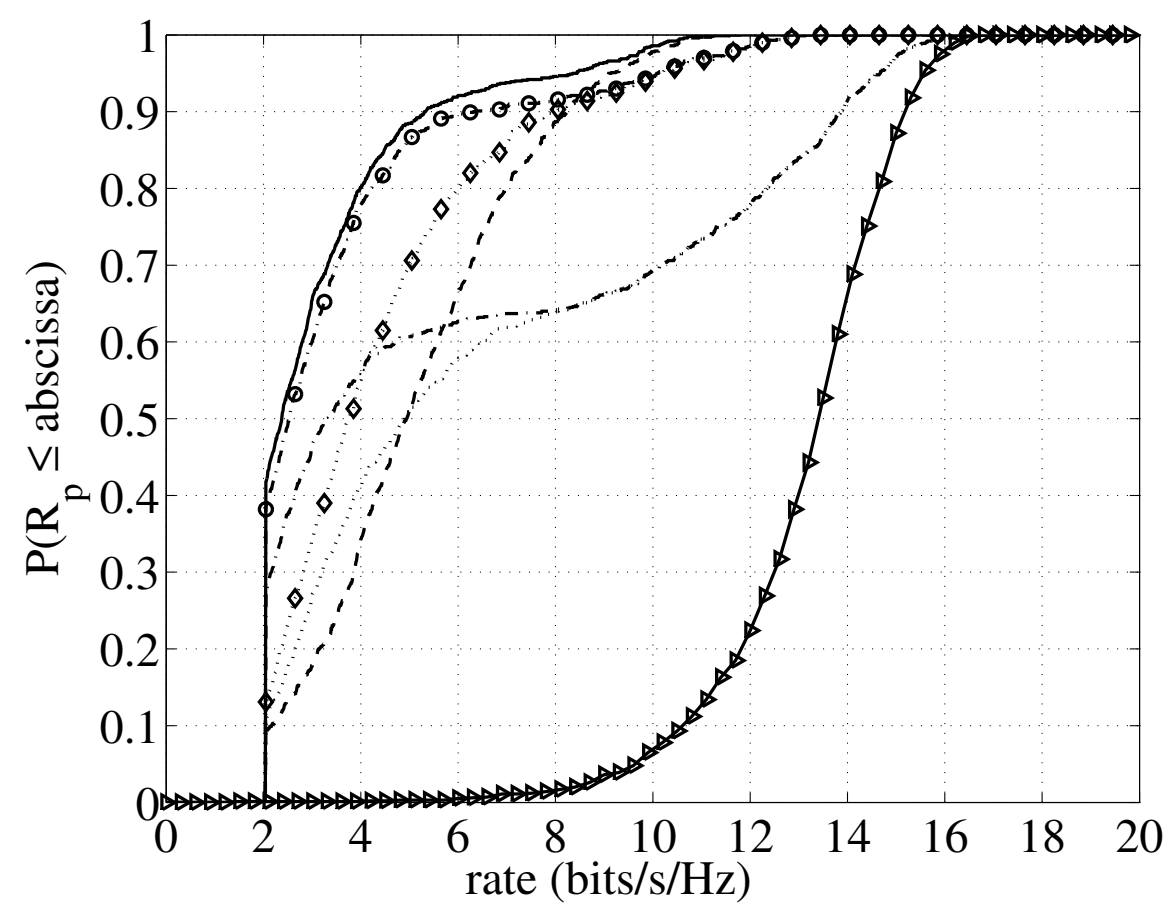

Figure 5.7: $R_{\mathrm{p}}$ CDF with perfect CSI for Scenario $C, \gamma_{\mathrm{T}}=5 \mathrm{~dB}$.

the PU's performance but adversely affects the SU's performance. Therefore, from the SU's point of view, the SU sum rate maximisation problem is the preferred option.

As stated previously, Scenario $C$ is solved using the high SINR approximation method. It is also possible to solve it using the more general condensation method. In Fig. 5.8 we compare the solution of the SU sum rate maximisation problem with SU QoS constraints ((5.8a)-(5.8d)) obtained using the two methods. From the results, it is evident that the high SINR approximation provides a fairly accurate approximation of the problem.

In Fig. 5.9 we compare the results of our proposed SU and system sum rate maximisation problems without SU QoS constraints ((5.8a) $-(5.8 \mathrm{c})$ and $(5.9 \mathrm{a}-5.9 \mathrm{~b}))$ against the ad hoc power allocation methods of [215]. We see that the ad hoc allocation methods are outperformed by the methods proposed in this chapter. We have only shown results for Scenario $A$ since similar results are obtained for Scenarios $B$ and $C$.

In Fig. 5.10 we compare the results of our feasibility detection for Scenario A against the brute force method of selecting the optimum set of SUs. As expected, the brute force method outperforms the proposed method; however, the performance improvement comes at a price of greatly increased computational complexity. Although suboptimal, the proposed feasibility detection method is an efficient method of improving the SU performance.

Figs. 5.11 and 5.12 show $R_{\Sigma}$ and $R_{\mathrm{p}}$ rate CDFs for Scenario $B$ with $\gamma_{\mathrm{T}}=5$ $\mathrm{dB}$ when channel uncertainties exist. The legend of Fig. 5.11 is applicable to Fig. 


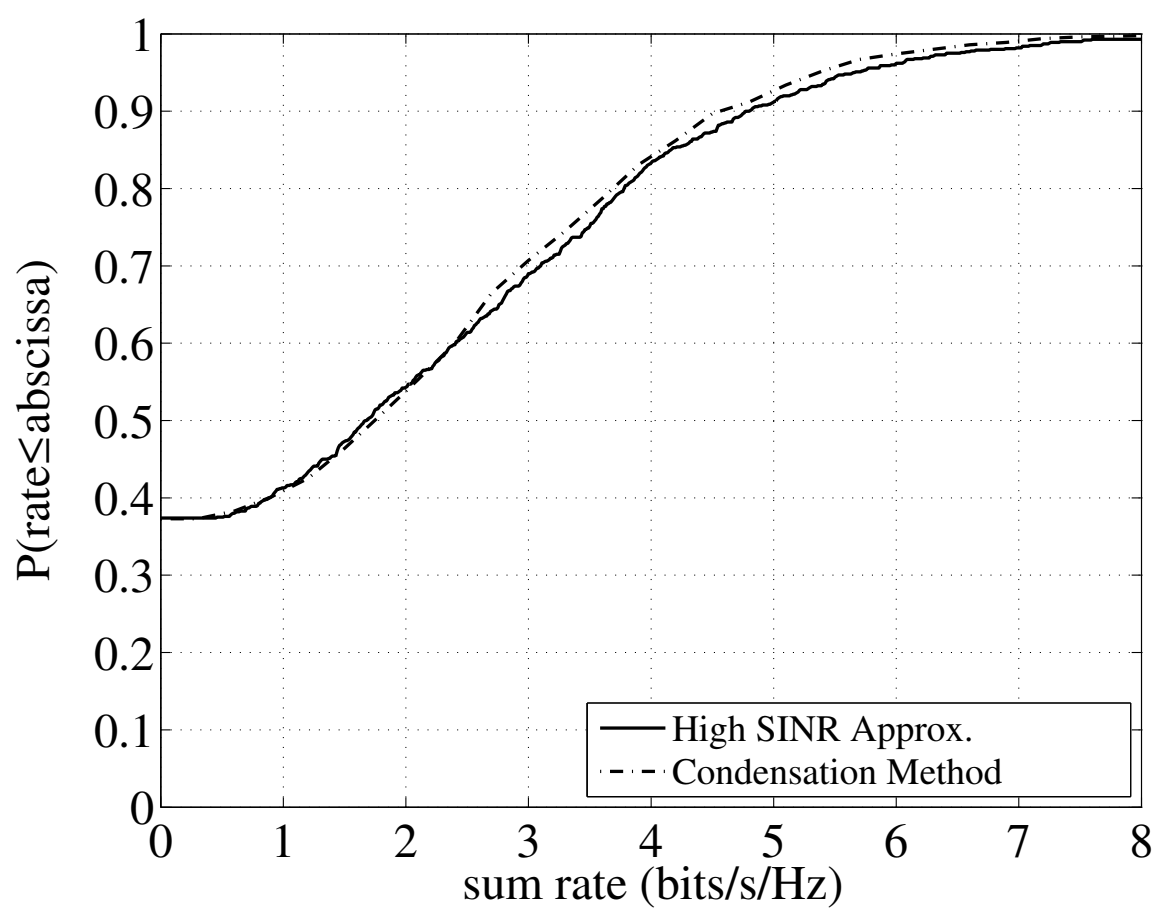

Figure 5.8: Sum rate CDF (SU QoS imposed) comparison of high SINR approximation and condensation method for Scenario $C, \gamma_{\mathrm{T}}=5 \mathrm{~dB}$.

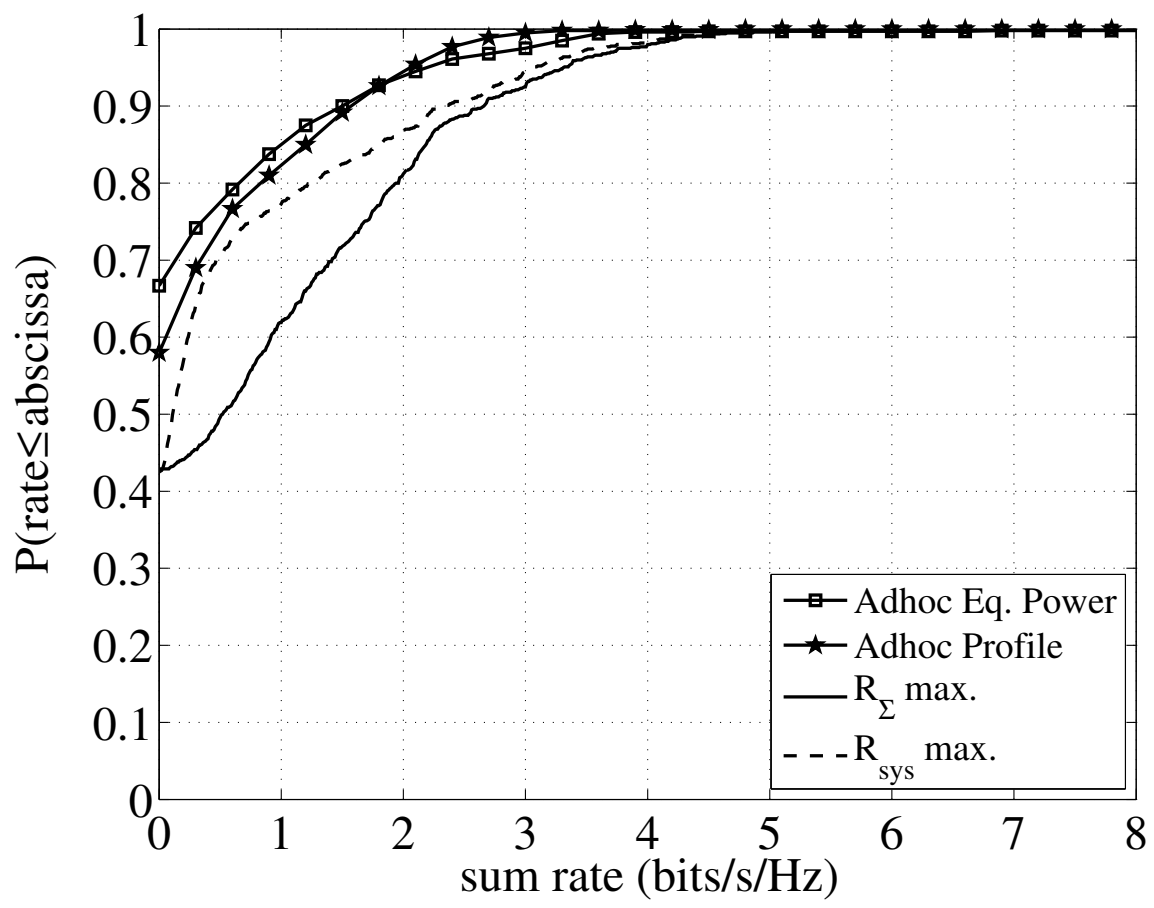

Figure 5.9: Sum rate CDF comparison of proposed method and ad hoc methods for Scenario $A, \gamma_{\mathrm{T}}=5 \mathrm{~dB}$. 


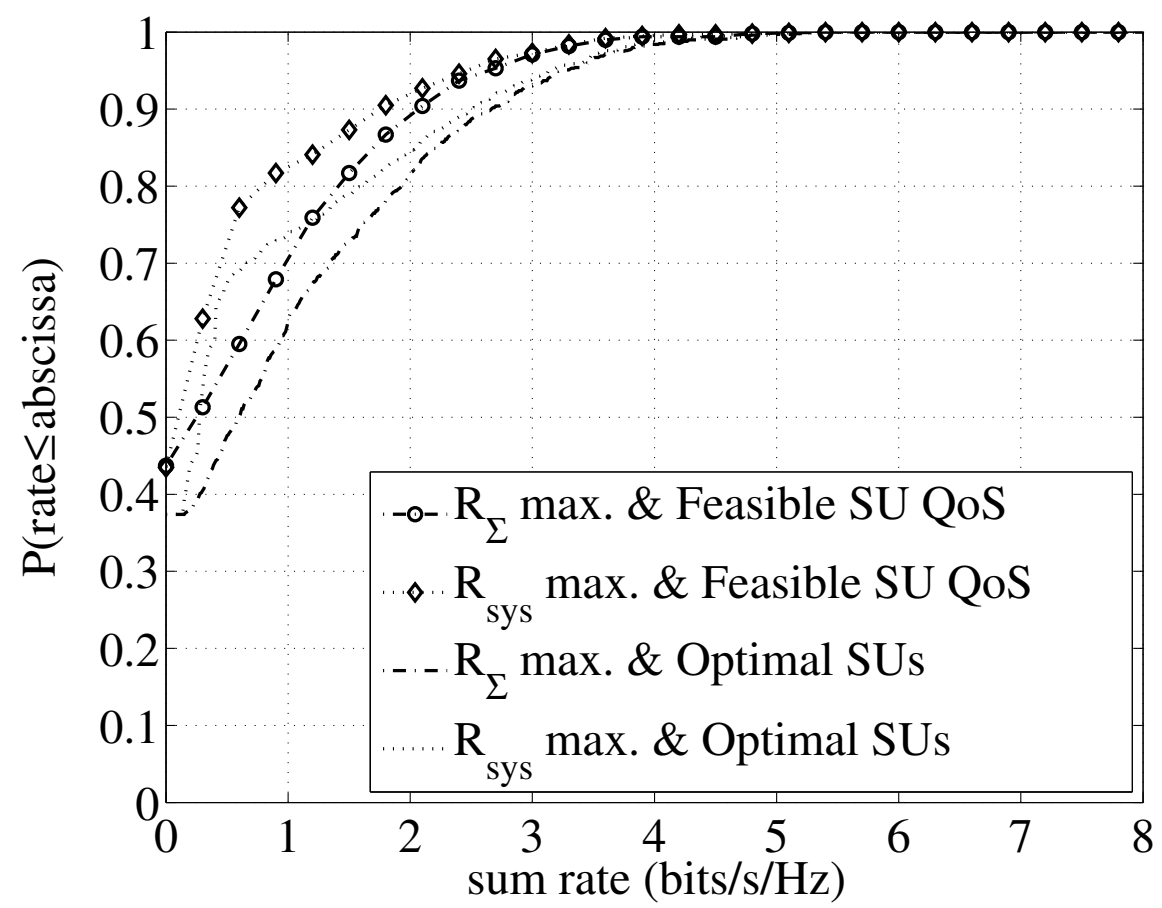

Figure 5.10: Sum rate CDF comparison of proposed feasibility detection and brute force methods for Scenario $A, \gamma_{\mathrm{T}}=5 \mathrm{~dB}$.

5.12. We only show results for Scenario $B$, since results obtained for Scenario $C$ similar to Scenario B. Results for Scenario $A$ are not shown because, due to unfavourable channel conditions, the PU's outage probability constraint is never satisfied and no SUs are able to access the channel, i.e, there is a $100 \%$ SU blocking probability for all optimisation problems. From Fig. 5.11, it is clear that there is a significant loss in the SU's performance compared to when perfect CSI is available, for instance, the median $R_{\Sigma}$ obtained using the robust $\mathrm{SU}$ sum rate maximisation problem without SU QoS constraints ((5.21a)-(5.21c)) is less than half of that obtained using its full CSI counterpart (problem (5.8a)-(5.8c)). Feasibility detection is again seen to reduce the SU blocking probability. Fig. 5.12 shows the distribution of $\gamma_{\mathrm{p}}$ under channel uncertainties. The inset shows the section between 0 $\mathrm{dB}-10 \mathrm{~dB}$ in greater detail and confirms that all optimisation problems attain an outage probability (probability PU's SINR is below $5 \mathrm{~dB}$ ) of $5 \%$ or less. From the SU's point of view, when channel uncertainties exist, the robust SU sum rate maximisation problem with feasibility detection ((5.24)) and the robust SU sum rate maximisation problem without SU QoS constraints ((5.21a)-(5.21c)) are the preferred options depending on whether the SU's QoS constraints are required or not.

Figs. 5.13-5.16 plot the mean $R_{\Sigma}$ as a function of $\gamma_{\mathrm{T}}$ for problems that impose SU's QoS constraints. From Figs. 5.13 and 5.14 it can be seen that feasibility detection significantly improves the SU's performance in all three scenarios when accurate CSI is available. Fig. 5.16 shows that when perfect CSI is not available, 


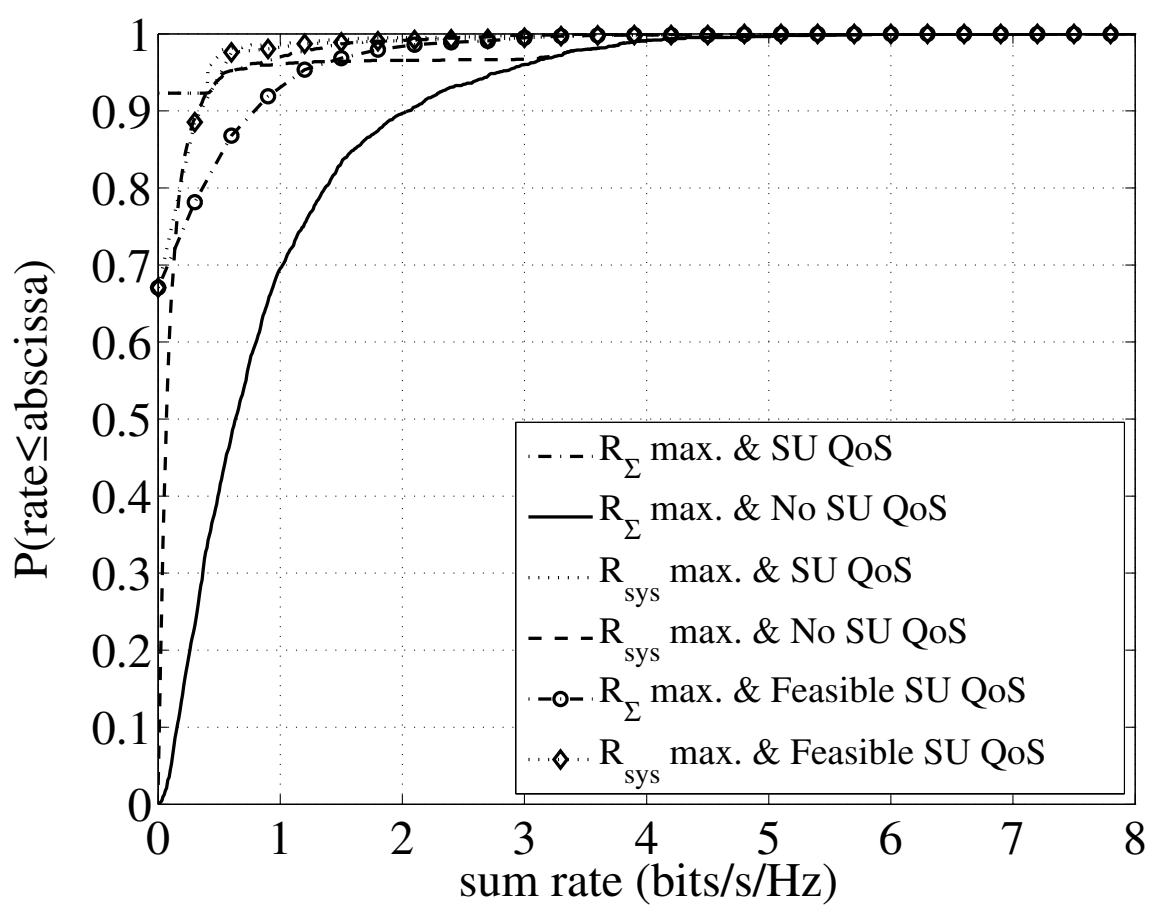

Figure 5.11: Sum rate CDF under channel uncertainties for Scenario $B, \gamma_{\mathrm{T}}=5$ $\mathrm{dB}, \mathrm{P}_{\mathrm{o}, \max }=5 \%$. The same legend is used in Fig. 5.12.

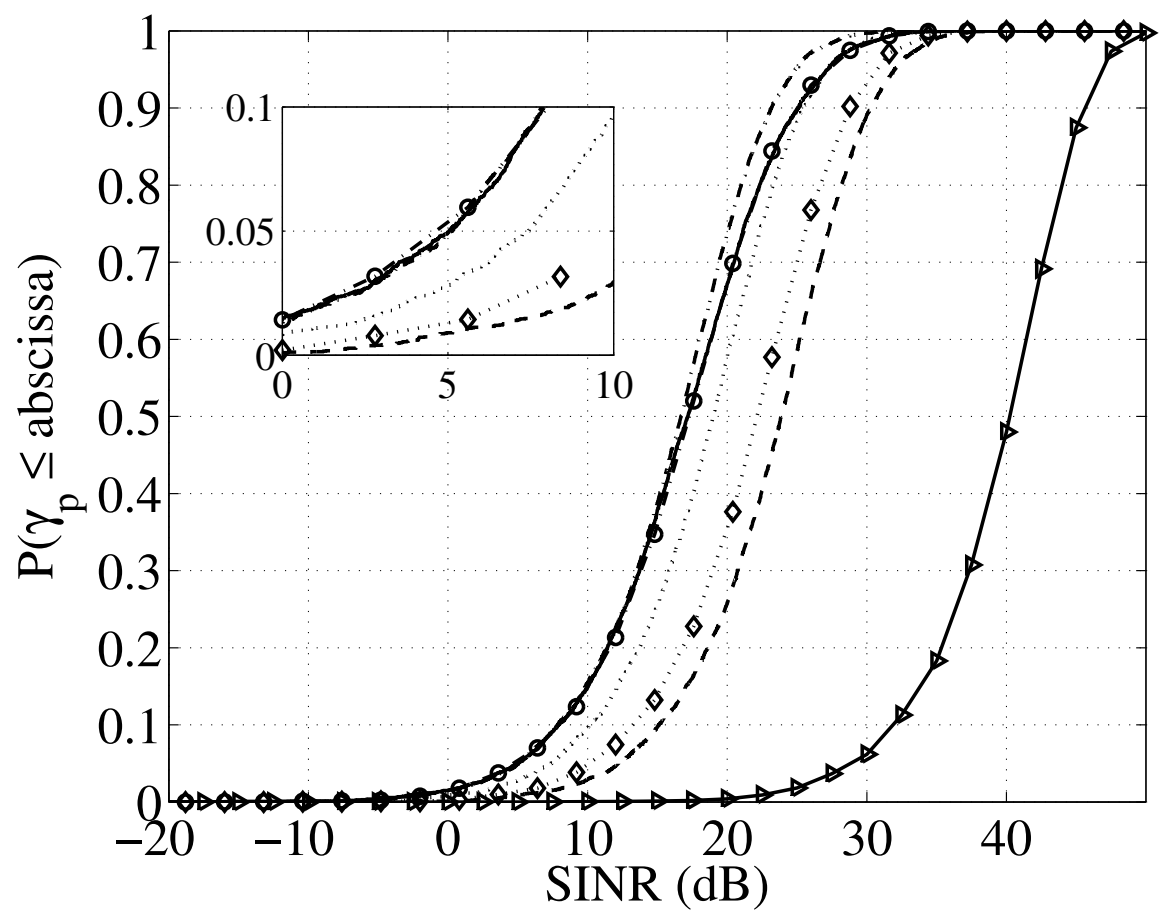

Figure 5.12: $\gamma_{\mathrm{p}}$ CDF under channel uncertainties for Scenario $B, \gamma_{\mathrm{T}}=5 \mathrm{~dB}$, $\mathrm{P}_{\mathrm{o}, \max }=5 \%$. 


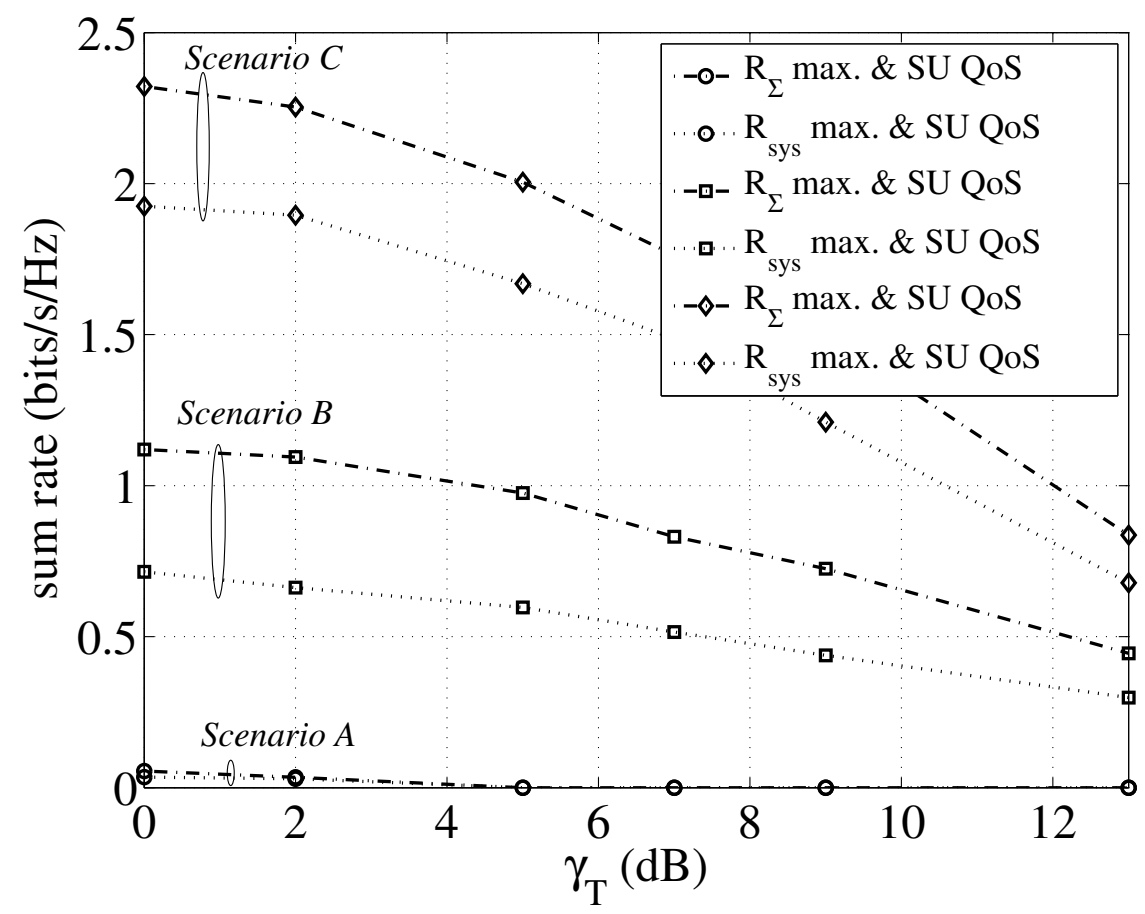

Figure 5.13: Mean sum rate when SU QoS are imposed as a function of $\gamma_{\mathrm{T}}$ with perfect CSI.

feasibility detection results in significant improvement of the SU's performance for Scenarios $B$ and $C$ while minor gains for lower values of $\gamma_{\mathrm{T}}$ are seen for Scenario $A$. Figs. 5.15 and 5.16 highlight the fact that unavailability of accurate CSI adversely affects the SU's performance, where practically no SU communications are possible in a high interference scenario. This can be improved if the PU relaxes its QoS requirements, either through a reduction of $\gamma_{\mathrm{T}}$ or an increase of $\mathrm{P}_{\mathrm{o} \text {,max }}$ or a combination of both. As expected, accurate knowledge of $\mathrm{PU}_{\mathrm{Tx}}$ to $\mathrm{PU}_{\mathrm{Rx}}$ and $\mathrm{SU}_{\mathrm{Tx}} \mathrm{S}$ to $\mathrm{PU}_{\mathrm{Rx}}$ links are crucial to the $\mathrm{SU}$ 's performance and large $\mathrm{SU}$ performance losses are expected if accurate CSI of these links is not available.

\subsection{Summary}

In this chapter, we have formulated the $\mathrm{SU}$ power allocation problem in a $\mathrm{CR}$ system as a GP and obtained rate CDFs in various channel conditions. We have included the effect of the PU's transmission in our formulations and studied the problem in both high and low SINR scenarios. It has been demonstrated that considering the system sum rate in the optimisation problem, in some circumstances, results in improved PU performance without a significant penalty in the SU's sum rate. Optimisation strategies for different channel conditions have been presented. Furthermore, we have presented a novel method of detecting and removing infeasible SU's QoS constraints from the SU power allocation problem that results in 


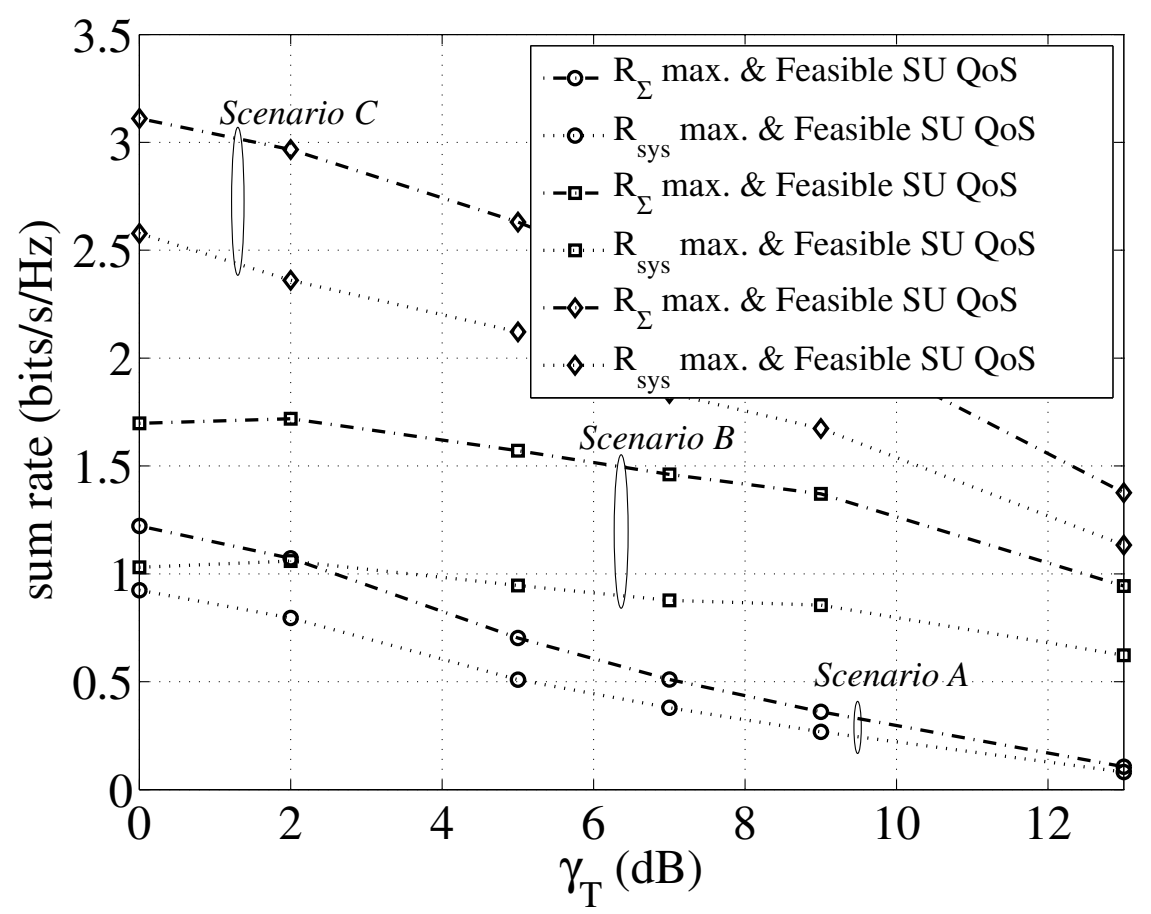

Figure 5.14: Mean sum rate as a function of $\gamma_{\mathrm{T}}$ with perfect CSI and feasibility detection.

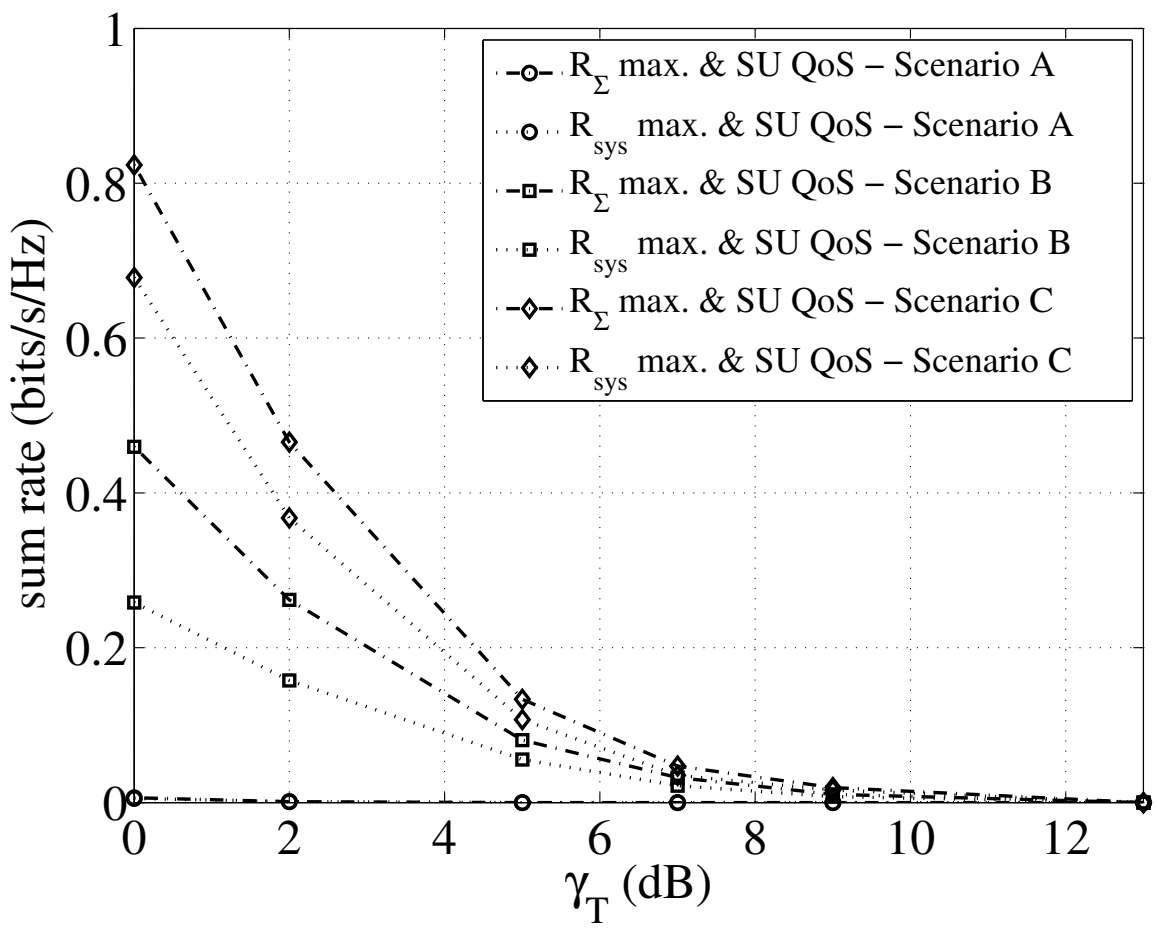

Figure 5.15: Mean sum rate when SU QoS are imposed as a function of $\gamma_{\mathrm{T}}$ under channel uncertainties, $\mathrm{P}_{\mathrm{o}, \max }=5 \%$. 


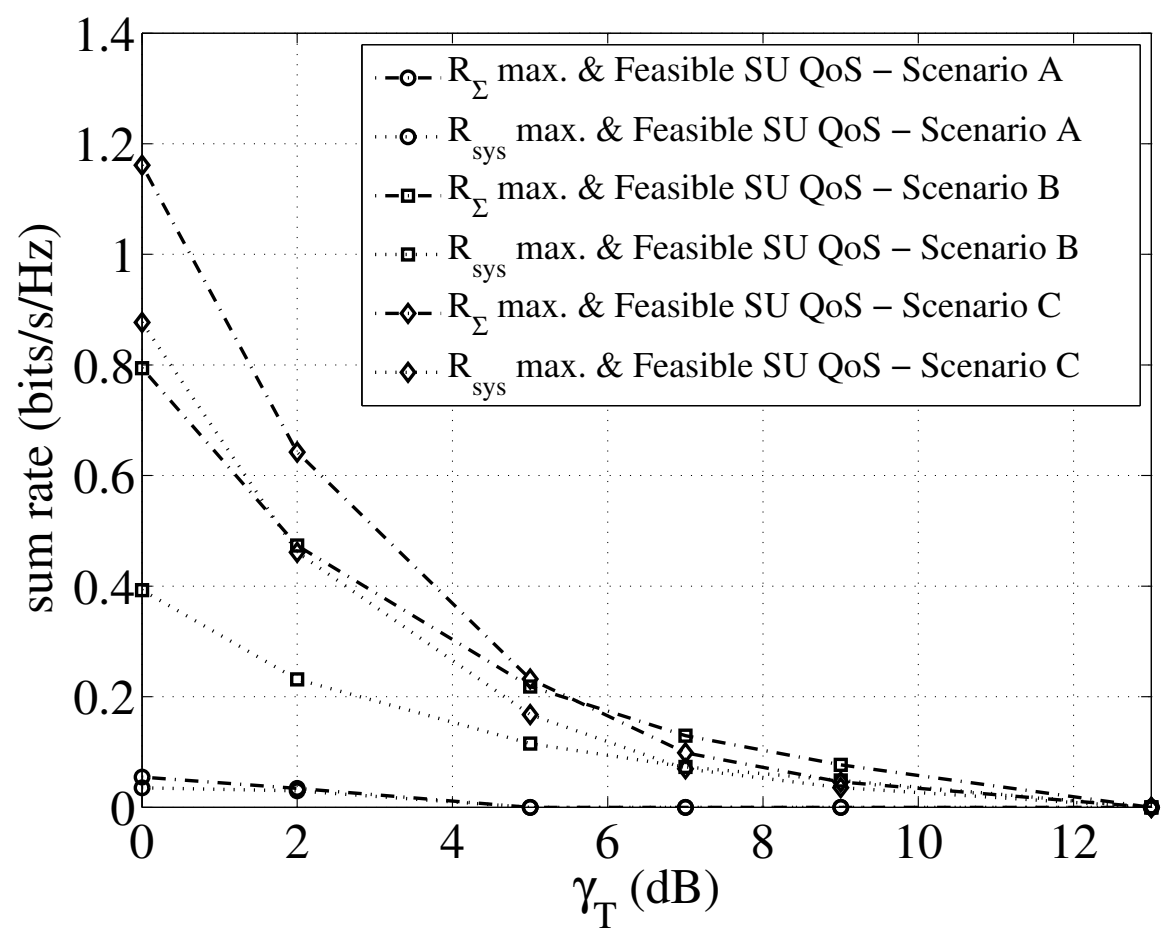

Figure 5.16: Mean sum rate as a function of $\gamma_{\mathrm{T}}$ under channel uncertainties and feasibility detection, $\mathrm{P}_{\mathrm{o}, \max }=5 \%$

considerably improved SU performance. A robust SU power allocation problem under channel uncertainties by considering a PU outage probability constraint has been presented. The results quantify the intuitive importance of $\mathrm{PU}_{\mathrm{Tx}}$ to $\mathrm{PU}_{\mathrm{Rx}}$ and $\mathrm{SU}_{\mathrm{Tx}} \mathrm{S}$ to $\mathrm{PU}_{\mathrm{Rx}} \mathrm{CSI}$ and large $\mathrm{SU}$ performance losses are encountered if accurate CSI of these links is not available. 



\section{Chapter 6}

\section{Robust Cognitive Radio Cooperative Beamforming}

This chapter considers an underlay CR relay network consisting of a cognitive source, a cognitive destination and a number of cognitive relay nodes that share spectrum with a primary transmitter and receiver. Due to poor channel conditions, the cognitive source is unable to communicate directly with the cognitive destination and hence employs the cognitive relays for assistance. We assume that perfect CSI for all links is not available to the CR. Under the assumption of partial and imperfect CSI at the CR system, we propose new robust CR cooperative relay beamformers where either the total relay transmit power or the cognitive destination SINR is optimised subject to a constraint on the primary receiver outage probability. We formulate the robust total relay power minimisation and the cognitive destination SINR maximisation optimisation problems as a convex second order cone program and a semidefinite program, respectively. Cumulative distribution functions of primary receiver and cognitive destination receiver SINR for Rayleigh fading channels are presented.

\subsection{Introduction}

The performance of underlay CR systems can be significantly improved by the use of multiple antennas. These performance improvements can also be realised by system employing multiple single antenna relay nodes through a technique known as cooperative relaying $[99,201,269,277]$. Geographically distributed relay nodes are cooperatively able to form a virtual antenna array and provide increased gains in capacity through distributed beamforming.

Recently, there has been increasing attention to the use of cooperative beamforming in CR systems (see, e.g., [6,96,140]). The relay nodes are typically deployed by the $\mathrm{CR}$ system to aid a $\mathrm{SU}$ transmitter $\left(\mathrm{SU}_{\mathrm{Tx}}\right)$ to communicate with a distant $\mathrm{SU}$ receiver $\left(\mathrm{SU}_{\mathrm{Rx}}\right)$ when the link between the $\mathrm{SU}_{\mathrm{Tx}}$ and $\mathrm{SU}_{\mathrm{Rx}}$ is poor. 
Cooperative beamforming at the relays not only improves SU performance through beamforming but also allows more control over the interference generated at the $\mathrm{PU}_{\mathrm{Rx}}$. The best beamformer performance is obviously obtained when perfect/full CSI is available and the design of CR cooperative relay beamformers under this assumption has been studied in $[6,96,140]$. In practical communication systems, this assumption may be over idealistic as perfect CSI for all links is rarely available. Channel estimation errors, limited CSI feedback and outdated channel estimates are some of the sources of the imperfections. The design of worst-case robust cooperative beamformers that are less susceptible to these imperfections have been investigated in $[232,245,277]$. Unfortunately, solutions obtained through the worstcase approach can be overly conservative because the true probability of worst-case errors may be extremely low [38].

In a CR relay network, CSI of the $\mathrm{PU}_{\mathrm{Tx}}$ to $\mathrm{PU}_{\mathrm{Rx}}$ and $\mathrm{SU}$ relays $\left(\mathrm{SU}_{\mathrm{Rls}}\right)$ to $\mathrm{PU}_{\mathrm{Rx}}$ is generally difficult to acquire and some level of cooperation with the PU system may be required. The level of cooperation determines the quality of the CSI that is available to the SU. Therefore, in this chapter, we consider a CR relay network where only partial and imperfect CSI of the $\mathrm{PU}_{\mathrm{Tx}}$ to $\mathrm{PU}_{\mathrm{Rx}}$ and the $\mathrm{SU}$ relays to $\mathrm{PU}_{\mathrm{Rx}}$ links is available to the $\mathrm{CR}$ system. We propose new robust $\mathrm{CR}$ cooperative relay beamformers where either the total relay transmit power or the cognitive destination SINR is optimised subject to a $\mathrm{PU}_{\mathrm{Rx}}$ outage probability constraint.

The contributions of this chapter are as follows.

- We first formulate the CR relay cooperative beamforming problem under the assumption of full CSI at the CR system as total relay power minimisation and cognitive destination SINR maximisation problems.

- We show that the total relay power minimisation and the cognitive destination SINR optimisation problems can be transformed into a convex second order cone program (SOCP) [29] and a convex semidefinite program (SDP), respectively.

- We present robust beamformers that guarantee a certain $\mathrm{PU}_{\mathrm{Rx}}$ outage probability for the scenarios where partial CSI is available for the $\mathrm{PU}_{\mathrm{Tx}}$ to $\mathrm{PU}_{\mathrm{Rx}}$ link and

1. full CSI is available for all other links;

2. partial CSI is available for the $\mathrm{SU}$ relays to $\mathrm{PU}_{\mathrm{Rx}}$ links and full CSI is available for all other links;

3. imperfect CSI is available for the $\mathrm{SU}$ relays to $\mathrm{PU}_{\mathrm{Rx}}$ links and full CSI is available for all other links. 


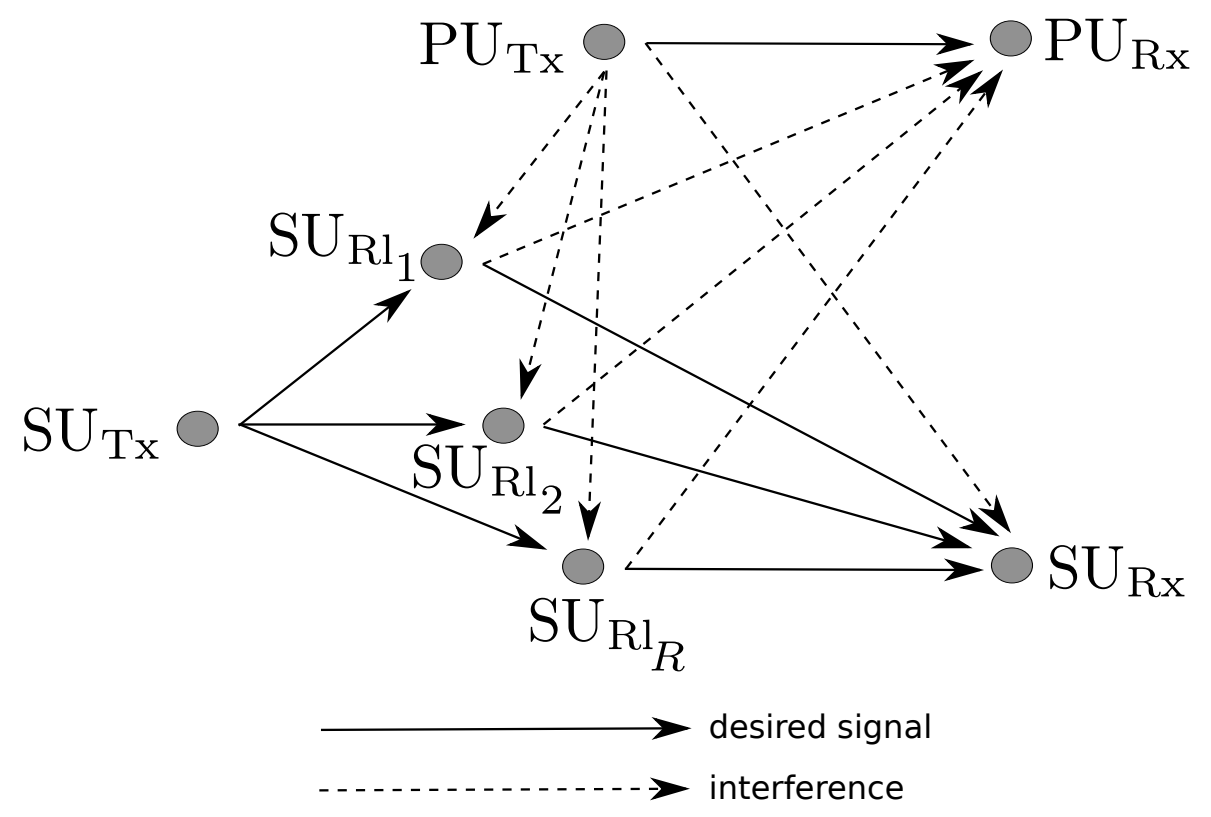

Figure 6.1: System Model

- We show that the robust total relay power minimisation and the robust cognitive destination SINR optimisation problems can be transformed into a convex SOCP [29] and a convex SDP, respectively.

The performance resulting from the optimisation problems outlined above is demonstrated by means of SINR CDFs for flat Rayleigh channels.

In this chapter, we assume both i) the proposed optimisation problems are solved by a central SU processing unit; and ii) a dedicated link, such as that in a distributed antenna system [191,266], between this central SU processing unit and each relay node exists. Note that the techniques developed in chapter can also be applied to a system with a single relay node equipped with multiple antennas.

\subsection{System Model}

Consider a $\mathrm{CR}$ relay network which consists of a $\mathrm{SU}_{\mathrm{Tx}}, \mathrm{SU}_{\mathrm{Rx}}, R \mathrm{SU}_{\mathrm{Rl}}$ nodes and a $\mathrm{PU}_{\mathrm{Tx}}$ and $\mathrm{PU}_{\mathrm{Rx}}$ pair, as shown in Fig. 6.1. We assume that due to poor channel conditions between the $\mathrm{SU}_{\mathrm{Tx}}$ and $\mathrm{SU}_{\mathrm{Rx}}$, there is no reliable link between them. Hence, the $\mathrm{SU}_{\mathrm{Tx}}$ employs the $\mathrm{SU}_{\mathrm{Rls}}$ to communicate with the $\mathrm{SU}_{\mathrm{Rx}}$. Since the $\mathrm{PU}$ and SU systems use the same frequency band, the $\mathrm{PU}_{\mathrm{Rx}}$ experiences interference from the $\mathrm{SU}_{\mathrm{Rl}}$ transmissions and both $\mathrm{SU}_{\mathrm{Rl}}$ and $\mathrm{SU}_{\mathrm{Rx}}$ experience interference from the $\mathrm{PU}_{\mathrm{Tx}}$ transmissions. Furthermore, we assume that the link between the $\mathrm{SU}_{\mathrm{Tx}}$ and $\mathrm{PU}_{\mathrm{Rx}}$ is poor and the $\mathrm{SU}_{\mathrm{Tx}}$ signal is sufficiently attenuated at the $\mathrm{PU}_{\mathrm{Rx}}$ to be ignored. Including the $\mathrm{SU}_{\mathrm{Tx}}$ interference at the $\mathrm{PU}_{\mathrm{Rx}}$ changes the solutions but not their structure, hence, it has been omitted for simplicity. Each transmitter and receiver in the system is assumed to be equipped with a single antenna. 
All links in the network are assumed to be independent, point-to-point, flat Rayleigh fading channels. The channel coefficients of the $\mathrm{PU}_{\mathrm{Tx}}$ to $\mathrm{PU}_{\mathrm{Rx}}, \mathrm{PU}_{\mathrm{Tx}}$ to $\mathrm{SU}_{\mathrm{Rl}} i, \mathrm{PU}_{\mathrm{Tx}}$ to $\mathrm{SU}_{\mathrm{Rx}}, \mathrm{SU}_{\mathrm{Tx}}$ to $\mathrm{SU}_{\mathrm{Rl}} i, \mathrm{SU}_{\mathrm{Rl}} i$ to $\mathrm{SU}_{\mathrm{Rx}}$ and $\mathrm{SU}_{\mathrm{Rl}} i$ to $\mathrm{PU}_{\mathrm{Rx}}$ links are denoted by $h_{\mathrm{pp}}, h_{\mathrm{pr}}^{(i)}, h_{\mathrm{ps}}, h_{\mathrm{sr}}^{(i)}, h_{\mathrm{rs}}^{(i)}$ and $h_{\mathrm{rp}}^{(i)}$, respectively. The instantaneous channel powers of these links are represented by $g_{\mathrm{pp}}=\left|h_{\mathrm{pp}}\right|^{2}, g_{\mathrm{pr}}^{(i)}=\left|h_{\mathrm{pr}}^{(i)}\right|^{2}, g_{\mathrm{ps}}=\left|h_{\mathrm{ps}}\right|^{2}$, $g_{\mathrm{sr}}^{(i)}=\left|h_{\mathrm{sr}}^{(i)}\right|^{2}, g_{\mathrm{rs}}^{(i)}=\left|h_{\mathrm{rs}}^{(i)}\right|^{2}$ and $g_{\mathrm{rp}}^{(i)}=\left|h_{\mathrm{rp}}^{(i)}\right|^{2}$. The channel powers are independent exponentially distributed random variables and have the means: $\Omega_{\mathrm{pp}}=\mathbb{E}\left\{g_{\mathrm{pp}}\right\}$, $\Omega_{\mathrm{pr}}^{(i)}=\mathbb{E}\left\{g_{\mathrm{pr}}^{(i)}\right\}, \Omega_{\mathrm{ps}}=\mathbb{E}\left\{g_{\mathrm{ps}}\right\}, \Omega_{\mathrm{sr}}^{(i)}=\mathbb{E}\left\{g_{\mathrm{sr}}^{(i)}\right\}, \Omega_{\mathrm{rs}}^{(i)}=\mathbb{E}\left\{g_{\mathrm{rs}}^{(i)}\right\}$ and $\Omega_{\mathrm{rp}}^{(i)}=\mathbb{E}\left\{g_{\mathrm{rp}}^{(i)}\right\}$.

We consider a secondary system that utilises a two-step AF protocol. During the first step, the $\mathrm{SU}_{\mathrm{Tx}}$ broadcasts the signal $\sqrt{P_{\mathrm{S}}} s_{\mathrm{S}}$ to the relays, where $P_{\mathrm{s}}$ is the $\mathrm{SU}_{\mathrm{Tx}}$ transmit power and $s_{\mathrm{S}}$ the information symbol. Simultaneously, the PU $\mathrm{U}_{\mathrm{Tx}}$ transmits the signal $\sqrt{P_{\mathrm{p}}} s_{\mathrm{p}}^{(1)}$, where $P_{\mathrm{p}}$ is the $\mathrm{PU}_{\mathrm{Tx}}$ transmit power and $s_{\mathrm{p}}^{(1)}$ the information symbol. We assume that $\mathbb{E}\left\{\left|s_{\mathrm{s}}\right|^{2}\right\}=\mathbb{E}\left\{\left|s_{\mathrm{p}}^{(1)}\right|^{2}\right\}=1$. The signal received at the $i$ th relay is given by

$$
x_{i}=\underbrace{\sqrt{P_{\mathrm{s}}} s_{\mathrm{s}} h_{\mathrm{sr}}^{*(i)}}_{\text {wanted signal }}+\underbrace{\sqrt{P_{\mathrm{p}}} s_{\mathrm{p}}^{(1)} h_{\mathrm{pr}}^{*(i)}+n_{\mathrm{r}}^{(i)}}_{\text {interference }+ \text { noise }},
$$

where $n_{\mathrm{r}}^{(i)}$ is the AWGN with a variance of $\sigma_{\mathrm{r}}^{2}$ at the $i$ th relay.

During the second step, the $i$ th relay transmits the signal

$$
\begin{aligned}
y_{i} & =x_{i} w_{i} \\
& =\sqrt{P_{\mathrm{s}}} s_{\mathrm{s}} h_{\mathrm{sr}}^{*(i)} w_{i}+\sqrt{P_{\mathrm{p}}} s_{\mathrm{p}}^{(1)} h_{\mathrm{pr}}^{*(i)} w_{i}+n_{\mathrm{r}}^{(i)} w_{i},
\end{aligned}
$$

where $w_{i}$ is the complex beamforming weight applied by the $i$ th relay. During this time, the $\mathrm{PU}_{\mathrm{Tx}}$ transmits the signal $\sqrt{P_{\mathrm{p}}} s_{\mathrm{p}}^{(2)}$, where $s_{\mathrm{p}}^{(2)}$ is the information symbol and is assumed to be different from that transmitted in the first step. We assume that $\mathbb{E}\left\{\left|s_{\mathrm{p}}^{(2)}\right|^{2}\right\}=1$. At the $\mathrm{SU}_{\mathrm{Rx}}$, the received signal can be expressed as

$$
\begin{aligned}
z_{s}= & \sum_{i=1}^{R} y_{i} h_{\mathrm{rs}}^{*(i)}+\sqrt{P_{\mathrm{p}}} s_{\mathrm{p}}^{(2)} h_{\mathrm{ps}}^{*} \\
= & \underbrace{\sqrt{P_{\mathrm{s}}} s_{\mathrm{s}}\left[\mathbf{h}_{\mathrm{sr}} \odot \mathbf{h}_{\mathrm{rs}}\right]^{H} \mathbf{w}}_{\text {wanted signal }}+\underbrace{\left[\mathbf{n}_{\mathrm{r}} \odot \mathbf{h}_{\mathrm{rs}}\right]^{H} \mathbf{w}+n_{\mathrm{s}}}_{\text {noise }} \\
& +\underbrace{\sqrt{P_{\mathrm{p}}} s_{\mathrm{p}}^{(2)} h_{\mathrm{ps}}^{*}+\sqrt{P_{\mathrm{p}}} s_{\mathrm{p}}^{(1)}\left[\mathbf{h}_{\mathrm{pr}} \odot \mathbf{h}_{\mathrm{rs}}\right]^{H} \mathbf{w}}_{\text {interference }},
\end{aligned}
$$

and that at the $\mathrm{PU}_{\mathrm{Rx}}$ as

$$
\begin{aligned}
z_{p} & =\sqrt{P_{\mathrm{p}}} s_{\mathrm{p}}^{(2)} h_{\mathrm{pp}}+\sum_{i=1}^{R} y_{i} h_{\mathrm{rp}}^{(i)} \\
& =\underbrace{\sqrt{P_{\mathrm{p}}} s_{\mathrm{p}}^{(2)} h_{\mathrm{pp}}}_{\text {wanted signal }}+\underbrace{\left[\mathbf{n}_{\mathrm{r}} \odot \mathbf{h}_{\mathrm{rp}}\right]^{H} \mathbf{w}+n_{\mathrm{p}}}_{\text {noise }}
\end{aligned}
$$




$$
+\underbrace{\sqrt{P_{\mathrm{s}}} s_{\mathrm{s}}\left[\mathbf{h}_{\mathrm{sr}} \odot \mathbf{h}_{\mathrm{rp}}\right]^{H} \mathbf{w}}_{\text {SU interference }}+\underbrace{\sqrt{P_{\mathrm{p}}} s_{\mathrm{p}}^{(1)}\left[\mathbf{h}_{\mathrm{pr}} \odot \mathbf{h}_{\mathrm{rp}}\right]^{H} \mathbf{w}}_{\text {self interference }},
$$

where

$$
\begin{aligned}
& \mathbf{h}_{\mathrm{sr}} \triangleq\left[h_{\mathrm{sr}}^{(1)} h_{\mathrm{sr}}^{(2)} \ldots h_{\mathrm{sr}}^{(R)}\right]^{T} \\
& \mathbf{h}_{\mathrm{rs}} \triangleq\left[h_{\mathrm{rs}}^{(1)} h_{\mathrm{rs}}^{(2)} \ldots h_{\mathrm{rs}}^{(R)}\right]^{T} \\
& \mathbf{h}_{\mathrm{pr}} \triangleq\left[h_{\mathrm{pr}}^{(1)} h_{\mathrm{pr}}^{(2)} \ldots h_{\mathrm{pr}}^{(R)}\right]^{T} \\
& \mathbf{h}_{\mathrm{rp}} \triangleq\left[h_{\mathrm{rp}}^{(1)} h_{\mathrm{rp}}^{(2)} \ldots h_{\mathrm{rp}}^{(R)}\right]^{T} \\
& \mathbf{w} \triangleq\left[\begin{array}{llll}
w_{1} & w_{2} & \ldots & w_{R}
\end{array}\right]^{T} \\
& \mathbf{n}_{\mathrm{r}} \triangleq\left[\begin{array}{llll}
n_{r}^{(1)} n_{r}^{(2)} & \ldots & n_{r}^{(R)}
\end{array}\right]^{T}
\end{aligned}
$$

and $n_{\mathrm{s}}$ and $n_{\mathrm{p}}$ are AWGN with powers $\sigma_{\mathrm{s}}^{2}$ and $\sigma_{\mathrm{p}}^{2}$ at the $\mathrm{SU}_{\mathrm{Rx}}$ and $\mathrm{PU}_{\mathrm{Rx}}$, respectively. Note that the relays also retransmit the PU's signal, hence, the $\mathrm{PU}_{\mathrm{Rx}}$ also receives the $\mathrm{PU}_{\mathrm{Tx}}$ symbol from the first step, which is treated as self interference in our analysis.

By assuming that $s_{\mathrm{s}}, s_{\mathrm{p}}^{(1)}, s_{\mathrm{p}}^{(2)}, n_{\mathrm{r}}^{(i)} \forall i, n_{\mathrm{s}}$ and $n_{\mathrm{p}}$ are all uncorrelated from each other and perfect CSI is available, and therefore considering the channel coefficients as deterministic constants, the total relay transmit power can be expressed as

$$
\begin{aligned}
P_{T} & =\sum_{i=1}^{R} \mathbb{E}\left\{\left|y_{i}\right|^{2}\right\} \\
& =\mathbf{w}^{H} \mathbf{E} \mathbf{w},
\end{aligned}
$$

where

$$
\mathbf{E} \triangleq P_{\mathrm{s}} \operatorname{diag}\left(\left|\mathbf{h}_{\mathrm{sr}}\right|^{2}\right)+P_{\mathrm{p}} \operatorname{diag}\left(\left|\mathbf{h}_{\mathrm{pr}}\right|^{2}\right)+\sigma_{\mathrm{r}}^{2} \mathrm{I}
$$

The $i$ th relay's transmit power is given by $P_{\mathrm{Rl}}^{(i)}=\mathbf{E}_{i i}\left|w_{i}\right|^{2}$. The SINR at the $\mathrm{SU}_{\mathrm{Rx}}$ is expressed as

$$
\begin{aligned}
\gamma_{\mathrm{s}} & =\frac{P_{\mathrm{s}}\left|\left[\mathbf{h}_{\mathrm{sr}} \odot \mathbf{h}_{\mathrm{rs}}\right]^{H} \mathbf{w}\right|^{2}}{P_{\mathrm{p}}\left|h_{\mathrm{ps}}\right|^{2}+P_{\mathrm{p}}\left|\left[\mathbf{h}_{\mathrm{pr}} \odot \mathbf{h}_{\mathrm{rs}}\right]^{H} \mathbf{w}\right|^{2}+\sigma_{\mathrm{r}}^{2}\left\|\mathbf{h}_{\mathrm{rs}} \odot \mathbf{w}\right\|^{2}+\sigma_{\mathrm{s}}^{2}} \\
& =\frac{\mathbf{w}^{H} \mathbf{Q w}}{P_{\mathrm{p}}\left|h_{\mathrm{ps}}\right|^{2}+\mathbf{w}^{H}(\mathbf{R}+\mathbf{V}) \mathbf{w}+\sigma_{\mathrm{s}}^{2}},
\end{aligned}
$$

where

$$
\begin{aligned}
& \mathbf{Q} \triangleq P_{\mathrm{s}}\left[\mathbf{h}_{\mathrm{sr}} \odot \mathbf{h}_{\mathrm{rs}}\right]\left[\mathbf{h}_{\mathrm{sr}} \odot \mathbf{h}_{\mathrm{rs}}\right]^{H} \\
& \mathbf{R} \triangleq P_{\mathrm{p}}\left[\mathbf{h}_{\mathrm{pr}} \odot \mathbf{h}_{\mathrm{rs}}\right]\left[\mathbf{h}_{\mathrm{pr}} \odot \mathbf{h}_{\mathrm{rs}}\right]^{H} \\
& \mathbf{V} \triangleq \sigma_{\mathrm{r}}^{2} \operatorname{diag}\left(\left|\mathbf{h}_{\mathrm{rs}}\right|^{2}\right) .
\end{aligned}
$$


Using the following definition

$$
I_{p} \triangleq P_{\mathrm{s}}\left|\left[\mathbf{h}_{\mathrm{sr}} \odot \mathbf{h}_{\mathrm{rp}}\right]^{H} \mathbf{w}\right|^{2}+P_{\mathrm{p}}\left|\left[\mathbf{h}_{\mathrm{pr}} \odot \mathbf{h}_{\mathrm{rp}}\right]^{H} \mathbf{w}\right|^{2}+\sigma_{\mathrm{r}}^{2}\left\|\mathbf{h}_{\mathrm{rp}} \odot \mathbf{w}\right\|^{2}
$$

the SINR at the $\mathrm{PU}_{\mathrm{Rx}}$ can be expressed as

$$
\begin{aligned}
\gamma_{\mathrm{p}} & =\frac{P_{\mathrm{p}}\left|h_{\mathrm{pp}}\right|^{2}}{I_{p}+\sigma_{\mathrm{p}}^{2}} \\
& =\frac{P_{\mathrm{p}}\left|h_{\mathrm{pp}}\right|^{2}}{\mathbf{w}^{H}(\mathbf{B}+\mathbf{C}+\mathbf{D}) \mathbf{w}+\sigma_{\mathrm{p}}^{2}},
\end{aligned}
$$

where

$$
\begin{aligned}
& \mathbf{B} \triangleq P_{\mathrm{s}}\left[\mathbf{h}_{\mathrm{sr}} \odot \mathbf{h}_{\mathrm{rp}}\right]\left[\mathbf{h}_{\mathrm{sr}} \odot \mathbf{h}_{\mathrm{rp}}\right]^{H} \\
& \mathbf{C} \triangleq P_{\mathrm{p}}\left[\mathbf{h}_{\mathrm{pr}} \odot \mathbf{h}_{\mathrm{rp}}\right]\left[\mathbf{h}_{\mathrm{pr}} \odot \mathbf{h}_{\mathrm{rp}}\right]^{H} \\
& \mathbf{D} \triangleq \sigma_{\mathrm{r}}^{2} \operatorname{diag}\left(\left|\mathbf{h}_{\mathrm{rp}}\right|^{2}\right) .
\end{aligned}
$$

To guarantee a certain level of quality-of-service (QoS) to the primary user, in our beamformer design formulations under the assumption of perfect CSI, we impose the $\mathrm{PU}_{\mathrm{Rx}}$ instantaneous SINR constraint $\gamma_{\mathrm{p}} \geq \gamma_{\mathrm{T}}$. This constraint is transformed into a probability based constraint in Section 6.4.

\subsection{Beamformer Optimisation}

In this section, we aim to find the optimum beamforming weight vector, w, such that either the total relay transmit power, $P_{T}$, is minimised or the SINR at the $\mathrm{SU}_{\mathrm{Rx}}, \gamma_{\mathrm{s}}$, is maximised while maintaining the $\mathrm{PU} \mathrm{Rx}_{\mathrm{RoS}}$ above the threshold $\gamma_{\mathrm{T}}$. In the case where the total relay transmit power is minimised, we also impose a minimum SINR threshold, $\gamma_{\mathrm{s} \text {,min }}$, on the $\mathrm{SU}_{\mathrm{Rx}}$. This represents a practical limitation on the $\mathrm{SU}_{\mathrm{Rx}}$ below which it fails to operate with acceptable performance. We also set individual maximum transmit power constraint, $P_{\mathrm{Rl} \text {,max }}^{(i)}$, on each relay node when maximising the $\mathrm{SU}_{\mathrm{Rx}} \mathrm{SINR}$. In practice, this constraint may be due either to regulatory or hardware limitations.

In our formulations, we assume that we are unable to control the PU's transmit power and the $\mathrm{PU}$ transmits at a constant power of $P_{\mathrm{p}}$. In this section, the beamformers are designed under the assumption that perfect CSI for all links are available at the SU system. This allows us to obtain fundamental limits on performance. However, in practice, the channel would need to be estimated, hence the performance results obtained in this section provide an upper bound. In Section 6.4, we consider the case when perfect CSI is not available. 


\subsubsection{Relay Power Minimisation}

Using (6.5), the total relay transmit power minimisation problem can be mathematically represented as

$$
\begin{array}{cl}
\underset{\mathbf{w}}{\min } & \mathbf{w}^{H} \mathbf{E w} \\
\text { s.t. } & \gamma_{\mathrm{p}} \geq \gamma_{\mathrm{T}}, \\
& \gamma_{\mathrm{s}} \geq \gamma_{\mathrm{s}, \min } .
\end{array}
$$

Similar to other beamforming problems (see, for example [99]), it can easily be shown that constraint (6.8c) is satisfied with equality at the optimum, for otherwise the optimum w could be scaled down to satisfy the constraint with equality, hence decreasing the objective function and contradicting optimality. Problem (6.8) is a non-convex optimisation problem; however, it can be reformulated into a convex optimisation problem. We observe that neither the objective function nor the constraints change if the beamforming vector undergoes a phase rotation. Thus, $\left[\mathbf{h}_{\mathrm{sr}} \odot \mathbf{h}_{\mathrm{rs}}\right]^{H} \mathbf{w}$ can be chosen to be real without the loss of generality [25, 148]. The SINR constraints (6.8b) and (6.8c) become

$$
\begin{aligned}
& P_{\mathrm{p}}\left|h_{\mathrm{pp}}\right|^{2} \geq \gamma_{\mathrm{T}}\left\|\begin{array}{c}
\sqrt{P_{\mathrm{s}}}\left[\mathbf{h}_{\mathrm{sr}} \odot \mathbf{h}_{\mathrm{rp}}\right]^{H} \mathbf{w} \\
\sqrt{P_{\mathrm{p}}}\left[\mathbf{h}_{\mathrm{pr}} \odot \mathbf{h}_{\mathrm{rp}}\right]^{H} \mathbf{w} \\
\sigma_{\mathrm{r}}\left[\mathbf{h}_{\mathrm{rp}} \odot \mathbf{w}\right] \\
\sigma_{\mathrm{p}}
\end{array}\right\|_{2}^{2} \\
& \left.P_{\mathrm{s}}\left(\left[\mathbf{h}_{\mathrm{sr}} \odot \mathbf{h}_{\mathrm{rs}}\right]\right]^{H} \mathbf{w}\right)^{2} \geq \gamma_{\mathrm{s}, \min }\left\|\begin{array}{c}
\sqrt{P_{\mathrm{p}}} h_{\mathrm{ps}} \\
\sqrt{P_{\mathrm{p}}\left[\mathbf{h}_{\mathrm{pr}} \odot \mathbf{h}_{\mathrm{rs}}\right]^{H} \mathbf{w}} \\
\sigma_{\mathrm{r}}\left[\mathbf{h}_{\mathrm{rs}} \odot \mathbf{w}\right] \\
\sigma_{\mathrm{s}}
\end{array}\right\|_{2}^{2}
\end{aligned}
$$

By taking the square root of constraints (6.9) and (6.10) the relay power minimisation problem can be restated as the following convex SOCP [29]

$$
\begin{array}{ll}
\min _{\mathbf{w}} & \mathbf{w}^{H} \mathbf{E w} \\
\text { s.t. } & \sqrt{P_{\mathrm{p}}\left|h_{\mathrm{pp}}\right|^{2}} \geq \sqrt{\gamma_{\mathrm{T}}} \| \begin{array}{c}
\sqrt{P_{\mathrm{s}}}\left[\mathbf{h}_{\mathrm{sr}} \odot \mathbf{h}_{\mathrm{rp}}\right]^{H} \mathbf{w} \\
\sqrt{P_{\mathrm{p}}}\left[\mathbf{h}_{\mathrm{pr}} \odot \mathbf{h}_{\mathrm{rp}}\right]^{H} \mathbf{w} \\
\sigma_{\mathrm{r}}\left[\mathbf{h}_{\mathrm{rp}} \odot \mathbf{w}\right] \\
\sigma_{\mathrm{p}}
\end{array} \\
\sqrt{P_{\mathrm{s}}}\left[\mathbf{h}_{\mathrm{sr}} \odot \mathbf{h}_{\mathrm{rs}}\right]^{H} \mathbf{w} \geq \sqrt{\gamma_{\mathrm{s}, \min }} & \sqrt{P_{\mathrm{p}}} h_{\mathrm{ps}} \\
\left.\sqrt{P_{\mathrm{p}}}\left[\mathbf{h}_{\mathrm{pr}} \odot \mathbf{h}_{\mathrm{rs}}\right]\right]^{H} \mathbf{w} \\
\sigma_{\mathrm{r}}\left[\mathbf{h}_{\mathrm{rs}} \odot \mathbf{w}\right] \\
\sigma_{\mathrm{s}}
\end{array} \|_{2} .
$$


In the interest of brevity, the further constraints $\Re\left\{\left[\mathbf{h}_{\mathrm{sr}} \odot \mathbf{h}_{\mathrm{rs}}\right]^{H} \mathbf{w}\right\} \geq 0$ and $\Im\left\{\left[\mathbf{h}_{\mathrm{sr}} \odot \mathbf{h}_{\mathrm{rs}}\right]^{H} \mathbf{w}\right\}=$ 0 , are not explicitly stated in any of the SOCPs in the following sections.

\subsubsection{Secondary Receiver SINR Maximisation}

In this section we formulate the $\mathrm{SU}_{\mathrm{Rx}}$ SINR maximisation problem and present three methods for solving the problem. In the first two methods, the problem is transformed into convex feasibility problems with second-order cone and semidefinite constraints, respectively. In both cases, the resulting problems are solved in an iterative manner. The third method transforms the problem into a linear-fractional program [29] and solves it without needing an iterative procedure. The $\mathrm{SU}_{\mathrm{Rx}} \mathrm{SINR}$ maximisation problem is expressed as

$$
\begin{array}{cl}
\max _{\mathbf{w}} & \frac{\mathbf{w}^{H} \mathbf{Q w}}{\mathbf{w}^{H}(\mathbf{R}+\mathbf{V}) \mathbf{w}+P_{\mathrm{p}}\left|h_{\mathrm{ps}}\right|^{2}+\sigma_{\mathrm{s}}^{2}} \\
\text { s.t. } & \mathbf{E}_{i i}\left|w_{i}\right|^{2} \leq P_{\mathrm{Rl}, \max }^{(i)}, \quad i=1 \ldots R \\
& \mathbf{w}^{H} \gamma_{\mathrm{T}}(\mathbf{B}+\mathbf{C}+\mathbf{D}) \mathbf{w}+\gamma_{\mathrm{T}} \sigma_{\mathrm{p}}^{2}-P_{\mathrm{p}}\left|h_{\mathrm{pp}}\right|^{2} \leq 0 .
\end{array}
$$

\section{Solution Using A Convex Feasibility Problem With SOC Constraints}

Problem (6.12) can be restated in the epigraph form [29] as

$$
\begin{array}{ll}
\max _{t, \mathbf{w}} & t \\
\text { s.t. } & \frac{\mathbf{w}^{H} \mathbf{Q w}}{\mathbf{w}^{H}(\mathbf{R}+\mathbf{V}) \mathbf{w}+P_{\mathrm{p}}\left|h_{\mathrm{ps}}\right|^{2}+\sigma_{\mathrm{s}}^{2}} \geq t, \\
& \mathbf{E}_{i i}\left|w_{i}\right|^{2} \leq P_{\mathrm{Rl}, \max }^{(i)}, \quad i=1 \ldots R \\
& \mathbf{w}^{H} \gamma_{\mathrm{T}}(\mathbf{B}+\mathbf{C}+\mathbf{D}) \mathbf{w}+\gamma_{\mathrm{T}} \sigma_{\mathrm{p}}^{2}-P_{\mathrm{p}}\left|h_{\mathrm{pp}}\right|^{2} \leq 0 .
\end{array}
$$

We again observe that neither the objective function nor the constraints change if the beamforming vector undergoes a phase rotation. Thus, $\left[\mathbf{h}_{\mathrm{sr}} \odot \mathbf{h}_{\mathrm{rs}}\right]^{H} \mathbf{w}$ can be chosen to be real without the loss of generality. Hence, problem (6.13) can be rewritten as

$$
\begin{array}{ll}
\max _{t, \mathbf{w}} & t \\
\text { s.t. } & \sqrt{P_{\mathrm{s}}}\left[\mathbf{h}_{\mathrm{sr}} \odot \mathbf{h}_{\mathrm{rs}}\right]^{H} \mathbf{w} \geq \sqrt{t} \| \begin{array}{c}
\sqrt{P_{\mathrm{p}}} h_{\mathrm{ps}} \\
\sqrt{P_{\mathrm{p}}}\left[\mathbf{h}_{\mathrm{pr}} \odot \mathbf{h}_{\mathrm{rs}}\right]{ }^{H} \mathbf{w} \\
\sigma_{\mathrm{r}}\left[\mathbf{h}_{\mathrm{rs}} \odot \mathbf{w}\right] \\
\sigma_{\mathrm{s}}
\end{array} \\
& \sqrt{P_{\mathrm{Rl}, \max }^{(i)} / \mathbf{E}_{i i}} \geq\left\|\begin{array}{c}
w_{i} \\
0
\end{array}\right\|_{2}, \quad i=1 \ldots R
\end{array}
$$




$$
\sqrt{P_{\mathrm{p}}\left|h_{\mathrm{pp}}\right|^{2}} \geq \sqrt{\gamma_{\mathrm{T}}}\left\|\begin{array}{c}
\sqrt{P_{\mathrm{s}}}\left[\mathbf{h}_{\mathrm{sr}} \odot \mathbf{h}_{\mathrm{rp}}\right]^{H} \mathbf{w} \\
\sqrt{P_{\mathrm{p}}}\left[\mathbf{h}_{\mathrm{pr}} \odot \mathbf{h}_{\mathrm{rp}}\right]^{H} \mathbf{w} \\
\sigma_{\mathrm{r}}\left[\mathbf{h}_{\mathrm{rp}} \odot \mathbf{w}\right] \\
\sigma_{\mathrm{p}}
\end{array}\right\|_{2}
$$

Due to coupling between the optimisation variables, constraint (6.14b) is a nonconvex constraint. However, as was seen in Section 3.3.4, for any fixed value of $t$ the set of feasible $\mathbf{w}$ is convex and hence the problem is quasi convex. Therefore, for some given $t$, problem (6.14) can be expressed as the following convex feasibility problem with SOCP constraints

$$
\begin{array}{ll}
\text { find } & \mathbf{w} \\
\text { s.t. } & (6.14 \mathrm{~b}),(6.14 \mathrm{c}) \text { and }(6.14 \mathrm{~d}) .
\end{array}
$$

The bisection method [29] can be used to solve problem (6.15) in an iterative manner.

\section{Solution Using A Convex Feasibility Problem With Linear and Semidef- inite Constraints}

Problem (6.12) can also be transformed into a SDP through the application of SDR. Using the definition $\mathbf{W} \triangleq \mathbf{w} \mathbf{w}^{H}$, it can be restated as

$$
\begin{array}{ll}
\max _{\mathbf{W}} & \frac{\operatorname{tr}(\mathbf{Q W})}{\operatorname{tr}((\mathbf{R}+\mathbf{V}) \mathbf{W})+P_{\mathrm{p}}\left|h_{\mathrm{ps}}\right|^{2}+\sigma_{\mathrm{s}}^{2}} \\
\text { s.t. } & \mathbf{E}_{i i} \mathbf{W}_{i i} \leq P_{\mathrm{Rl}, \max }^{(i)}, \quad i=1 \ldots R \\
& \gamma_{\mathrm{T}} \operatorname{tr}((\mathbf{B}+\mathbf{C}+\mathbf{D}) \mathbf{W})+\gamma_{\mathrm{T}} \sigma_{\mathrm{p}}^{2}-P_{\mathrm{p}}\left|h_{\mathrm{pp}}\right|^{2} \leq 0, \\
& \mathbf{W} \succeq 0, \\
& \operatorname{rank}(\mathbf{W})=1,
\end{array}
$$

and in the epigraph form as

$$
\begin{array}{ll}
\max _{\mathbf{W}, t} & t \\
\text { s.t. } & \operatorname{tr}((\mathbf{Q}-t(\mathbf{R}+\mathbf{V})) \mathbf{W}) \geq\left(P_{\mathrm{p}}\left|h_{\mathrm{ps}}\right|^{2}+\sigma_{\mathrm{s}}^{2}\right) t \\
& \operatorname{rank}(\mathbf{W})=1, \\
& \mathbf{W} \succeq 0,(6.16 \mathrm{~b}) \text { and }(6.16 \mathrm{c}) .
\end{array}
$$

Due to the non-convex constraints $(6.17 \mathrm{~b})$ and $(6.17 \mathrm{c})$, problem $(6.17)$ is a nonconvex optimisation problem. We apply the idea of SDR and relax problem (6.17) by removing the non-convex rank-one constraint $(6.17 \mathrm{c})$. The resulting relaxed problem is still non-convex as constraint $(6.17 \mathrm{~b})$ remains to be dealt with. As in 
the previous solution, for any fixed value of $t$ the set of feasible $\mathbf{W}$ is convex and hence the relaxed problem is quasi convex. Therefore, for some given $t$, problem (6.17) can be expressed as the following convex feasibility problem

$$
\begin{array}{ll}
\text { find } & \mathbf{W} \\
\text { s.t. } & \mathbf{W} \succeq 0,(6.17 \mathrm{~b}),(6.16 \mathrm{~b}) \text { and }(6.16 \mathrm{c}) .
\end{array}
$$

Problem (6.18) can be solved using the bisection method. Upon completion of the bisection algorithm, one needs to recover the optimum beamforming vector, $\mathbf{w}^{*}$, from $\mathbf{W}$. If $\mathbf{W}$ is rank-one, then $\mathbf{w}^{*}$ can be chosen to be the principal eigenvector of $\mathbf{W}$. For the case where $\mathbf{W}$ has rank higher than one, the well known Gaussian randomisation technique [146] can be used to recover a good rank-one approximation. However, in our extensive numerical simulations, we have never obtained a solution that had a rank higher than one. This behaviour is explained by the fact that problem (6.12) can also be solved as a convex feasibility problem with SOC constraints which does not involve any relaxation. Therefore, since another solution method exits, in this case the relaxed problem turns out to be equivalent to the original problem.

\section{Solution Using Linear-fractional Program}

To develop the third method of solving problem (6.12), we observe that the relaxed form of problem (6.16) has the same structure as a linear-fractional program which was introduced in Section 2.4.2. Hence, the Charnes-Cooper transformation can be used to solve it efficiently without needing an iterative procedure. To proceed, we first define the pair

$$
\begin{aligned}
\tilde{\mathbf{W}} & =\frac{\mathbf{W}}{\operatorname{tr}((\mathbf{R}+\mathbf{V}) \mathbf{W})+P_{\mathrm{p}}\left|h_{\mathrm{ps}}\right|^{2}+\sigma_{\mathrm{s}}^{2}}, \\
t & =\frac{1}{\operatorname{tr}((\mathbf{R}+\mathbf{V}) \mathbf{W})+P_{\mathrm{p}}\left|h_{\mathrm{ps}}\right|^{2}+\sigma_{\mathrm{s}}^{2}} .
\end{aligned}
$$

Using these definitions, the relaxed form of problem (6.16) can be stated as

$$
\begin{aligned}
\max _{\tilde{\mathbf{W}}, t} & \operatorname{tr}(\mathbf{Q} \tilde{\mathbf{W}}) \\
\text { s.t. } & \mathbf{E}_{i i} \tilde{\mathbf{W}}_{i i} \leq t P_{\mathrm{Rl}, \max }^{(i)}, \quad i=1 \ldots R \\
& \gamma_{\mathrm{T}} \operatorname{tr}((\mathbf{B}+\mathbf{C}+\mathbf{D}) \tilde{\mathbf{W}})+t\left(\gamma_{\mathrm{T}} \sigma_{\mathrm{p}}^{2}-P_{\mathrm{p}}\left|h_{\mathrm{pp}}\right|^{2}\right) \leq 0, \\
& \tilde{\mathbf{W}} \succeq 0, \\
& \operatorname{tr}((\mathbf{R}+\mathbf{V}) \tilde{\mathbf{W}})+t\left(P_{\mathrm{p}}\left|h_{\mathrm{ps}}\right|^{2}+\sigma_{\mathrm{s}}^{2}\right)=1, \\
& t \geq 0 .
\end{aligned}
$$


Problem (6.19) is a convex optimisation problem and can be solved using interior point methods. After solving this problem, the beamforming matrix is obtained by dividing $\tilde{\mathbf{W}}$ by $t$, i.e., $\mathbf{W}=\tilde{\mathbf{W}} / t$. The optimum beamforming vector, $\mathbf{w}^{*}$, is given by the principal eigenvector of $\mathbf{W}$.

\subsection{Robust Beamformer Optimisation}

So far we have assumed that perfect CSI of all links is available at the SU system. Unfortunately, in practise, perfect CSI for all links is seldom available and the assumption of perfect CSI may be unrealistic. For our analysis, we assume that the channels for the $\mathrm{SU}_{\mathrm{Tx}}$ to $\mathrm{SU}_{\mathrm{Rl}}$ and $\mathrm{SU}_{\mathrm{Rl}}$ to $\mathrm{SU}_{\mathrm{Rx}}$ links are accurately known through the SU's channel estimation procedure and those between the $\mathrm{PU}_{\mathrm{Tx}}$ and $\mathrm{SU}_{\mathrm{Rl}}$ can be accurately measured, for example, through knowledge of the PU pilot symbols. In this section we formulate a number of robust optimisation problems based on varying levels of uncertainty in the CSI of $\mathrm{PU}_{\mathrm{Tx}}$ to $\mathrm{PU}_{\mathrm{Rx}}$ and $\mathrm{SU}_{\mathrm{Rl}}$ to $\mathrm{PU}_{\mathrm{Rx}}$ links. In a cognitive radio system, this may correspond to the level of cooperation between the primary and secondary systems. Generally, CSI of the $\mathrm{PU}_{\mathrm{Tx}}$ to $\mathrm{PU}_{\mathrm{Rx}}$ link would be the most difficult to obtain since this link is fully isolated from the SU system. The SU would have to rely on the PU to provide this information and the CSI quality would depend on the level of cooperation between the two systems. In our robust beamformer formulations, we assume that the SU system has only partial CSI for the $\mathrm{PU}_{\mathrm{Tx}}$ to $\mathrm{PU}_{\mathrm{Rx}}$ link, specifically, we assume that only the mean channel power, $\Omega_{\mathrm{pp}}$, of this link is provided by the PU. CSI of the $\mathrm{SU}_{\mathrm{Rl}}$ to $\mathrm{PU}_{\mathrm{Rx}}$ link would also be difficult to acquire and cooperation with the $\mathrm{PU}$ would be needed. However, if the PU system had a bidirectional link, then the SU could estimate the CSI of the $\mathrm{PU}_{\mathrm{Rx}}$ to $\mathrm{SU}_{\mathrm{Rl}}$ link when the $\mathrm{PU}_{\mathrm{Rx}}$ assumes the role of a transmitter. In this section, we design robust beamformers based on the quality of the CSI of this link that is available to the SU. We focus on three levels of quality: i) perfect CSI; ii) imperfect CSI estimate; and iii) incomplete CSI in the form of mean channel powers.

In our formulation we consider the PU outage probability as a QoS parameter. The outage probability constraint is generally referred to as a soft constraint and as shown in [38], tends to be more flexible than a worst-case constraint. In the system under consideration, outage occurs when the PU SINR, $\gamma_{\mathrm{p}}$, falls below the PU SINR threshold, $\gamma_{\mathrm{T}}$. The outage probability is expressed as

$$
\begin{aligned}
P_{\mathrm{o}} & =\operatorname{Pr}\left\{\gamma_{\mathrm{p}} \leq \gamma_{\mathrm{T}}\right\} \\
& =\operatorname{Pr}\left\{\frac{P_{\mathrm{p}}\left|h_{\mathrm{pp}}\right|^{2}}{\mathbf{w}^{H}(\mathbf{B}+\mathbf{C}+\mathbf{D}) \mathbf{w}+\sigma_{\mathrm{p}}^{2}} \leq \gamma_{\mathrm{T}}\right\} .
\end{aligned}
$$

Hence, given a maximum allowable outage probability, $\mathrm{P}_{\mathrm{o}, \max }$, constraints $(6.11 \mathrm{~b})$ 
and $(6.12 \mathrm{c})$ are replaced with

$$
\operatorname{Pr}\left\{\frac{P_{\mathrm{p}}\left|h_{\mathrm{pp}}\right|^{2}}{\mathbf{w}^{H}(\mathbf{B}+\mathbf{C}+\mathbf{D}) \mathbf{w}+\sigma_{\mathrm{p}}^{2}} \leq \gamma_{\mathrm{T}}\right\} \leq \mathrm{P}_{\mathrm{o}, \max } .
$$

\subsubsection{Partial CSI Availability for the $\mathrm{PU}_{\mathrm{Tx}}$ to $\mathrm{PU}_{\mathrm{Rx}}$ Link}

In this section, we assume that perfect CSI is available for all links except for the $\mathrm{PU}_{\mathrm{Tx}}$ to $\mathrm{PU}_{\mathrm{Rx}}$ link. We assume that only the mean channel power, $\Omega_{\mathrm{pp}}$, of the $\mathrm{PU}_{\mathrm{Tx}}$ to $\mathrm{PU}_{\mathrm{Rx}}$ link is available, i.e., instantaneous channel realisation is not available. Since $h_{\mathrm{pp}}$ is a zero-mean Gaussian random variable, $\left|h_{\mathrm{pp}}\right|^{2}$ is exponentially distributed and therefore the outage probability can be expressed as

$$
\mathrm{P}_{\mathrm{o}}=1-\exp \left(-\frac{\gamma_{\mathrm{T}}\left(\mathbf{w}^{H}(\mathbf{B}+\mathbf{C}+\mathbf{D}) \mathbf{w}+\sigma_{\mathrm{p}}^{2}\right)}{P_{\mathrm{p}} \Omega_{\mathrm{pp}}}\right) .
$$

Using (6.22), the PU outage probability constraint (6.21) can then be stated as

$$
\mathbf{w}^{H}(\mathbf{B}+\mathbf{C}+\mathbf{D}) \mathbf{w}+\sigma_{\mathrm{p}}^{2}+\frac{P_{\mathrm{p}} \Omega_{\mathrm{pp}}}{\gamma_{\mathrm{T}}} \log \left(1-\mathrm{P}_{\mathrm{o}, \max }\right) \leq 0,
$$

or equivalently as the following SOCP constraint

$$
\sqrt{-P_{\mathrm{p}} \Omega_{\mathrm{pp}} \log \left(1-\mathrm{P}_{\mathrm{o}, \text { max }}\right)} \geq \sqrt{\gamma_{\mathrm{T}}}\left\|\begin{array}{c}
\left.\sqrt{P_{\mathrm{s}}}\left[\mathbf{h}_{\mathrm{sr}} \odot \mathbf{h}_{\mathrm{rp}}\right]\right]^{H} \mathbf{w} \\
\left.\sqrt{P_{\mathrm{p}}}\left[\mathbf{h}_{\mathrm{pr}} \odot \mathbf{h}_{\mathrm{rp}}\right]\right]^{H} \mathbf{w} \\
\sigma_{\mathrm{r}}\left[\mathbf{h}_{\mathrm{rp}} \odot \mathbf{w}\right] \\
\sigma_{\mathrm{p}}
\end{array}\right\|_{2}
$$

The robust $\mathrm{SU}_{\mathrm{Rl}}$ power minimisation problem in this scenario is therefore expressed as the following SOCP

$$
\min _{\mathbf{w}} \mathbf{w}^{H} \mathbf{E w}, \quad \text { s.t. } \quad(6.11 \mathrm{c}) \text { and }(6.24) \text {. }
$$

It is straightforward to show that the robust $\mathrm{SU}_{\mathrm{Rx}} \mathrm{SINR}$ maximisation problem is essentially the same as the relaxed form of problem (6.16) but with the instantaneous $\mathrm{PU}_{\mathrm{Rx}}$ SINR constraint (6.16c) replaced by the PU outage probability constraint as shown below

$$
\begin{array}{ll}
\max _{\mathbf{W}} & \frac{\operatorname{tr}(\mathbf{Q W})}{\operatorname{tr}((\mathbf{R}+\mathbf{V}) \mathbf{W})+P_{\mathrm{p}}\left|h_{\mathrm{ps}}\right|^{2}+\sigma_{\mathrm{s}}^{2}} \\
\text { s.t. } & (6.16 \mathrm{~b}) \text { and }(6.16 \mathrm{~d}), \\
& \operatorname{tr}((\mathbf{B}+\mathbf{C}+\mathbf{D}) \mathbf{W})+\sigma_{\mathrm{p}}^{2}+\frac{P_{\mathrm{p}} \Omega_{\mathrm{pp}}}{\gamma_{\mathrm{T}}} \log \left(1-\mathrm{P}_{\mathrm{o}, \max }\right) \leq 0 .
\end{array}
$$

The solution of problem (6.26) can be found using the method described in Section 6.3.2. 


\subsubsection{Partial CSI Availability for the $\mathrm{PU}_{\mathrm{Tx}}$ to $\mathrm{PU}_{\mathrm{Rx}}$ and $\mathrm{SU}_{\mathrm{Rl}}$ to $\mathrm{PU}_{\mathrm{Rx}}$ Links}

In this section, we assume that full CSI is available for all links except for the $\mathrm{PU}_{\mathrm{Tx}}$ to $\mathrm{PU}_{\mathrm{Rx}}$ and $\mathrm{SU}_{\mathrm{Rl}}$ to $\mathrm{PU}_{\mathrm{Rx}}$ links. We assume that only the mean channel powers, $\Omega_{\mathrm{pp}}$ and $\Omega_{\mathrm{rp}}^{(i)} \forall i$, of the $\mathrm{PU}_{\mathrm{Tx}}$ to $\mathrm{PU}_{\mathrm{Rx}}$ and $\mathrm{SU}_{\mathrm{Rl}}$ to $\mathrm{PU}_{\mathrm{Rx}}$ links are available. To proceed, we rewrite the outage probability expression as

$$
\mathrm{P}_{\mathrm{o}}=\operatorname{Pr}\left\{P_{\mathrm{p}}\left|h_{\mathrm{pp}}\right|^{2}-\gamma_{\mathrm{T}} \mathbf{w}^{H}(\mathbf{B}+\mathbf{C}+\mathbf{D}) \mathbf{w} \leq \gamma_{\mathrm{T}} \sigma_{\mathrm{p}}^{2}\right\}
$$

In (6.27), we see that we are dealing with a PDF that is given by the difference of two random variables, namely, $P_{\mathrm{p}}\left|h_{\mathrm{pp}}\right|^{2}$ and $\gamma_{\mathrm{T}} \mathbf{w}^{H}(\mathbf{B}+\mathbf{C}+\mathbf{D}) \mathbf{w}$. From Section 6.4.1, it is known that $P_{\mathrm{p}}\left|h_{\mathrm{pp}}\right|^{2}$ has an exponential distribution with a mean of $P_{\mathrm{p}} \Omega_{\mathrm{pp}}$. The probability distribution of $\gamma_{\mathrm{T}} \mathbf{w}^{H}(\mathbf{B}+\mathbf{C}+\mathbf{D}) \mathbf{w}$ can be found using the following lemma.

Lemma 6.4.1 If $\mathrm{x} \in \mathcal{C}^{R \times 1}$ is a zero-mean random vector with complex Gaussian elements and the covariance matrix $\boldsymbol{\Sigma}$, i.e. $\mathbf{x} \sim \mathcal{N}_{\mathcal{C}}(\mathbf{0}, \mathbf{\Sigma})$, then for any deterministic positive semidefinite Hermitian matrix $\mathbf{A} \in \mathcal{C}^{R \times R}$, the PDF of the random variable $\psi=\mathbf{x}^{H} \mathbf{A} \mathbf{x}, \psi \geq 0$, is given by

$$
f(\psi)=\left[\prod_{i=1}^{N} \lambda_{i}\right] \sum_{j=1}^{N} \frac{\exp \left(-\lambda_{j} \psi\right)}{\prod_{k=1, k \neq j}^{N}\left(\lambda_{k}-\lambda_{j}\right)},
$$

where $\lambda_{i}=1 / \Lambda_{i}$, and $\Lambda_{i}, i=1 \ldots N \leq R$ are the non-zero eigenvalues of $\boldsymbol{\Sigma} \mathbf{A}$. Note that this is precisely the distribution of the sum of $N$ exponentially distributed independent random variables, each with a mean of $\Lambda_{i}$.

\section{Proof}

Note that $\mathbf{x}^{H} \mathbf{A} \mathbf{x}$ is commonly known as a quadratic form in normal random variables [157]. We first define $\mathbf{y}=\boldsymbol{\Sigma}^{-\frac{1}{2}} \mathbf{X}$. It is easily verified that $\mathbf{y} \sim \mathcal{N}_{\mathcal{C}}(\mathbf{0}, \mathbf{I})$. Using an orthogonal $R \times R$ matrix $\mathbf{P}$ that diagonalises $\boldsymbol{\Sigma}^{\frac{1}{2}} \mathbf{A} \boldsymbol{\Sigma}^{\frac{1}{2}}$ or equivalently $\boldsymbol{\Sigma} \mathbf{A}$, i.e., $\mathbf{P}^{H} \mathbf{\Sigma} \mathbf{A P}=\operatorname{diag}\left(\Lambda_{1}, \Lambda_{2}, \ldots, \Lambda_{R}\right)$ and $\Lambda_{1}, \Lambda_{2}, \ldots, \Lambda_{R}$ are the eigenvalues of $\boldsymbol{\Sigma} \mathbf{A}, \psi$ can then be expressed as

$$
\begin{aligned}
\psi & =\mathbf{y}^{H} \boldsymbol{\Sigma}^{\frac{1}{2}} \mathbf{A} \boldsymbol{\Sigma}^{\frac{1}{2}} \mathbf{y}=\left(\mathbf{P}^{H} \mathbf{y}\right)^{H} \mathbf{P}^{H} \boldsymbol{\Sigma}^{\frac{1}{2}} \mathbf{A} \boldsymbol{\Sigma}^{\frac{1}{2}} \mathbf{P}\left(\mathbf{P}^{H} \mathbf{y}\right) \\
& =\operatorname{tr}\left(\operatorname{diag}\left(\Lambda_{1}, \Lambda_{2}, \ldots, \Lambda_{R}\right) \mathbf{P}^{H} \mathbf{y} \mathbf{y}^{H} \mathbf{P}\right) \\
& =\sum_{i=1}^{N} \Lambda_{i}\left|\left(\mathbf{P}^{H} \mathbf{y}\right)_{i}\right|^{2}
\end{aligned}
$$

where $N \leq R$ is the number of non-zero eigenvalues of $\boldsymbol{\Sigma} \mathbf{A}$ and $\left(\mathbf{P}^{H} \mathbf{y}\right)_{i}$ is the $i$ th element of the vector $\mathbf{P}^{H} \mathbf{y}$. Since $\mathbf{P}$ is an orthogonal matrix, it is easily shown that $\mathbf{P}^{H} \mathbf{y} \sim \mathcal{N}_{\mathcal{C}}(\mathbf{0}, \mathbf{I})$, and therefore, $\left|\left(\mathbf{P}^{H} \mathbf{y}\right)_{i}\right|^{2}$ is an exponentially distributed random 
variable with a mean of $\Lambda_{i}$. Hence, (6.29) is a sum of $N$ exponentially distributed independent random variables, each with a mean of $\Lambda_{i}$, whose PDF is given by (6.28). The derivation of the PDF of the sum of $N$ exponentially distributed independent random variables has appeared in many texts, we refer the interested reader to [117] and references therein. This completes the proof

From the definition of $\mathbf{B}$, we note that it can be expressed as $\mathbf{B}=\mathbf{b b}^{H}$, where $\mathbf{b}=\sqrt{P_{\mathrm{s}}}\left[\mathbf{h}_{\mathrm{sr}} \odot \mathbf{h}_{\mathrm{rp}}\right]$. Since $\mathbf{h}_{\mathrm{sr}}$ is known perfectly, it is treated as a deterministic constant in the following analysis. By defining $\mathbf{W}=\mathbf{w} \mathbf{w}^{H}$ we see that $\mathbf{w}^{H} \mathbf{B} \mathbf{w}=$ $\mathbf{b}^{H} \mathbf{W b}$. Using Lemma 6.4.1, and exploiting the fact that $\mathbf{W}$ is a rank-one matrix, we have that $\gamma_{\mathrm{T}} \mathbf{w}^{H} \mathbf{B w}$ is exponentially distributed with a mean of $\operatorname{tr}\left(\boldsymbol{\Sigma}_{B} \mathbf{W}\right)$, where $\boldsymbol{\Sigma}_{B}$ is the covariance matrix of $\sqrt{\gamma_{\mathrm{T}}} \mathbf{b}$ and is expressed as

$$
\begin{aligned}
\boldsymbol{\Sigma}_{B} & =\gamma_{\mathrm{T}} \mathbb{E}\{\mathbf{B}\} \\
& =\gamma_{\mathrm{T}} P_{\mathrm{s}} \operatorname{diag}\left(\boldsymbol{\Omega}_{\mathrm{rp}} \odot\left|\mathbf{h}_{\mathrm{sr}}\right|^{2}\right),
\end{aligned}
$$

where $\Omega_{\mathrm{rp}}=\left[\Omega_{\mathrm{rp}}^{1} \Omega_{\mathrm{rp}}^{2} \ldots \Omega_{\mathrm{rp}}^{R}\right]^{T}$. Similarly, $\gamma_{\mathrm{T}} \mathbf{w}^{H} \mathbf{C w}$ also has an exponential distribution with a mean of $\operatorname{tr}\left(\boldsymbol{\Sigma}_{C} \mathbf{W}\right)$, where $\boldsymbol{\Sigma}_{C}$ is the covariance matrix defined as

$$
\begin{aligned}
\boldsymbol{\Sigma}_{C} & =\gamma_{\mathrm{T}} \mathbb{E}\{\mathbf{C}\} \\
& =\gamma_{\mathrm{T}} P_{\mathrm{p}} \operatorname{diag}\left(\boldsymbol{\Omega}_{\mathrm{rp}} \odot\left|\mathbf{h}_{\mathrm{pr}}\right|^{2}\right) .
\end{aligned}
$$

Since $\mathbf{D}$ is a diagonal matrix, we have $\gamma_{\mathrm{T}} \mathbf{w}^{H} \mathbf{D w}=\gamma_{\mathrm{T}} \sigma_{\mathrm{r}}^{2} \sum_{i=1}^{R} \mathbf{W}_{i i}\left|h_{\mathrm{rp}}^{(i)}\right|^{2}$, which can be recognised as a sum of $R$ exponentially distributed random variables and, as such, the PDF is given by (6.28) whereby $N=R$ and $\lambda_{i}=1 /\left(\gamma_{\mathrm{T}} \sigma_{\mathrm{r}}^{2} \Omega_{\mathrm{rp}}^{(i)} \mathbf{W}_{i i}\right)$, $i=1 \ldots R$.

Finally, from the above analysis, we see that $\gamma_{\mathrm{T}} \mathbf{w}^{H}(\mathbf{B}+\mathbf{C}+\mathbf{D}) \mathbf{w}$ is the sum of $R+2$ exponentially distributed independent random variables and the PDF is once again given by (6.28), with $N=R+2, \lambda_{i}=1 /\left(\gamma_{\mathrm{T}} \sigma_{\mathrm{r}}^{2} \Omega_{\mathrm{rp}}^{(i)} \mathbf{W}_{i i}\right), i=1 \ldots R$, $\lambda_{R+1}=1 / \operatorname{tr}\left(\boldsymbol{\Sigma}_{B} \mathbf{W}\right)$ and $\lambda_{R+2}=1 / \operatorname{tr}\left(\boldsymbol{\Sigma}_{C} \mathbf{W}\right)$.

Having found the distribution of $\gamma_{\mathrm{T}} \mathbf{w}^{H}(\mathbf{B}+\mathbf{C}+\mathbf{D}) \mathbf{w}$, we are now in a position to evaluate the PU outage probability expression. We note that the PDF in (6.27) is that of a difference between an exponential random variable and the sum of $R+2$ exponentially distributed random variables, and therefore the outage probability is known to have the following form (see, for example, [117])

$$
\mathrm{P}_{\mathrm{o}}=1-\exp \left(-\frac{\gamma_{\mathrm{T}} \sigma_{\mathrm{p}}^{2}}{P_{\mathrm{p}} \Omega_{\mathrm{pp}}}\right) \prod_{i=1}^{R+2}\left(\frac{1}{1+\frac{1}{P_{\mathrm{p}} \Omega_{\mathrm{pp}} \lambda_{i}}}\right)
$$


where $\lambda_{i}$ is as defined previously. Using (6.32), the outage probability constraint can be expressed as

$$
\prod_{i=1}^{R+2}\left(1+\frac{1}{P_{\mathrm{p}} \Omega_{\mathrm{pp}} \lambda_{i}}\right) \leq \frac{\exp \left(-\frac{\gamma_{\mathrm{T}} \sigma_{\mathrm{p}}^{2}}{P_{\mathrm{p}} \Omega_{\mathrm{pp}}}\right)}{1-\mathrm{P}_{\mathrm{o}, \max }}
$$

An important observation in the above constraint is that it is dependent only on the diagonal elements of $\mathbf{W}$, i.e., dependent only on the beamformer transmit power. This is a fairly intuitive result since phase information of $\mathrm{SU}_{\mathrm{Rl}}$ to $\mathrm{PU}_{\mathrm{Rx}}$ link is not available and therefore, power control is the only degree of freedom available to the beamformer to control the amount of interference to the $\mathrm{PU}_{\mathrm{Rx}}$. Note that constraint (6.33) is non-convex (the term on the left hand side is in fact concave), and is difficult to handle. For this reason, we propose to use the geometric-arithmetic mean inequality and replace the left hand side of (6.33) with its upper bound. The geometric-arithmetic mean inequality is expressed as

$$
\prod_{i=1}^{R+2}\left(1+\frac{1}{P_{\mathrm{p}} \Omega_{\mathrm{pp}} \lambda_{i}}\right)^{\frac{1}{R+2}} \leq \frac{1}{R+2} \sum_{i=1}^{R+2}\left(1+\frac{1}{P_{\mathrm{p}} \Omega_{\mathrm{pp}} \lambda_{i}}\right)
$$

Using the inequality (6.34), the convex outage probability constraint is thus

$$
\sum_{i=1}^{R+2}\left(1+\frac{1}{P_{\mathrm{p}} \Omega_{\mathrm{pp}} \lambda_{i}}\right) \leq(R+2)\left(\frac{\exp \left(-\frac{\gamma_{\mathrm{T}} \sigma_{\mathrm{p}}^{2}}{P_{\mathrm{p}} \Omega_{\mathrm{pp}}}\right)}{1-\mathrm{P}_{\mathrm{o}, \mathrm{max}}}\right)^{\frac{1}{R+2}}
$$

By using the upper bound, the constraint is tightened and the ramifications of this on the optimum solution are discussed later in this section. Meanwhile, we present the robust optimisation problems by directly using (6.35).

Through straightforward manipulation, (6.35) can be rewritten as

$$
\begin{aligned}
& \frac{1}{P_{\mathrm{p}} \Omega_{\mathrm{pp}}} \mathbf{w}^{H}\left(\boldsymbol{\Sigma}_{B}+\boldsymbol{\Sigma}_{C}+\gamma_{\mathrm{T}} \sigma_{\mathrm{r}}^{2} \operatorname{diag}\left(\boldsymbol{\Omega}_{\mathrm{rp}}\right)\right) \mathbf{w} \\
& +(R+2)\left(1-\left(\frac{\exp \left(-\frac{\gamma_{\mathrm{T}} \sigma_{\mathrm{p}}^{2}}{P_{\mathrm{p}} \Omega_{\mathrm{pp}}}\right)}{1-\mathrm{P}_{\mathrm{o}, \max }}\right)^{\frac{1}{R+2}}\right) \leq 0,
\end{aligned}
$$

and the equivalent SOCP constraint is given by

$$
\sqrt{(R+2)\left(\left(\frac{\exp \left(-\frac{\gamma_{\mathrm{T}} \sigma_{\mathrm{p}}^{2}}{P_{\mathrm{p}} \Omega_{\mathrm{pp}}}\right)}{1-\mathrm{P}_{\mathrm{o}, \mathrm{max}}}\right)^{\frac{1}{R+2}}-1\right)} \geq \sqrt{\frac{\gamma_{\mathrm{T}}}{P_{\mathrm{p}} \Omega_{\mathrm{pp}}}}\left\|\begin{array}{c}
\sqrt{P_{\mathrm{s}}}\left[\boldsymbol{\Omega}_{\mathrm{rp}}^{1 / 2} \odot \mathbf{h}_{\mathrm{sr}} \odot \mathbf{w}\right] \\
\sqrt{P_{\mathrm{p}}}\left[\boldsymbol{\Omega}_{\mathrm{rp}}^{1 / 2} \odot \mathbf{h}_{\mathrm{pr}} \odot \mathbf{w}\right] \\
\sigma_{\mathrm{r}}\left[\boldsymbol{\Omega}_{\mathrm{rp}}^{1 / 2} \odot \mathbf{w}\right]
\end{array}\right\|_{2},
$$


where $\Omega_{\mathrm{rp}}^{1 / 2}$ is the element-wise square root of the vector $\boldsymbol{\Omega}_{\mathrm{rp}}$. The robust $\mathrm{SU}_{\mathrm{Rl}}$ power minimisation SOCP can therefore be expressed as

$$
\min _{\mathbf{w}} \mathbf{w}^{H} \mathbf{E w}, \quad \text { s.t. } \quad(6.11 \mathrm{c}) \text { and (6.37). }
$$

By directly using constraint (6.35), the robust $\mathrm{SU}_{\mathrm{Rx}}$ SINR maximisation problem can again be expressed as the convex feasibility problem

$$
\begin{array}{cl}
\max _{\mathbf{W}} & \frac{\operatorname{tr}(\mathbf{Q W})}{\operatorname{tr}((\mathbf{R}+\mathbf{V}) \mathbf{W})+P_{\mathrm{p}}\left|h_{\mathrm{ps}}\right|^{2}+\sigma_{\mathrm{s}}^{2}} \\
\text { s.t. } & (6.16 \mathrm{~b}),(6.16 \mathrm{~d}) \text { and }(6.35),
\end{array}
$$

which can be solved using the methods described in Section 6.3.2.

As previously mentioned, using the outage probability upper bound results in a tightening of the constraint. In the $\mathrm{SU}_{\mathrm{Rl}}$ power minimisation problem, this tightening may result in some feasible problems appearing infeasible. Likewise, the $\mathrm{SU}_{\mathrm{Rx}}$ SINR maximisation problem may become infeasible or the solution obtained may be sub-optimal since the power allocated to the beamformer would be less than what would have been allocated if the original constraint was used.

Recalling that at the optimum, constraint (6.11c) is satisfied with equality, it follows that if the robust $\mathrm{SU}_{\mathrm{Rl}}$ power minimisation problem (6.38) is feasible then the solution obtained is the optimum. This follows since any further reduction of relay transmit power would cause the $\mathrm{SU}_{\mathrm{Rx}} \mathrm{SINR}$ to fall below $\gamma_{\mathrm{s}, \mathrm{min}}$, thus violating the $\mathrm{SU}_{\mathrm{Rx}} \mathrm{SINR}$ and rendering the problem infeasible. On the other hand, if the problem is infeasible due to the tightened outage probability constraint then we need to determine if a feasible solution can be obtained by relaxing the constraint. This corresponds to finding the minimum relaxed outage probability specification, $\tilde{\mathrm{P}}_{\mathrm{o}, \max } \geq \mathrm{P}_{\mathrm{o} \text {,max }}$, that satisfies (6.33). An efficient iterative method utilising the bisection technique for finding $\tilde{\mathrm{P}}_{\mathrm{o}, \max }$ is presented in Algorithm 4. In each iteration, problem (6.38) is solved with the relaxed outage probability specification and the solution is used in (6.32) to calculate the exact outage probability, $\mathrm{P}_{\mathrm{o}}$, attained. $\mathrm{P}_{\mathrm{o}}$ is then compared with $\mathrm{P}_{\mathrm{o}, \max }$ to determine if (6.33) is satisfied. The iterations continue until the minimum $\tilde{\mathrm{P}}_{\mathrm{o} \text {,max }}$ is found. Note that the bisection algorithm requires the lower and upper limits of the search interval. The lower limit is $\mathrm{P}_{\mathrm{o}, \max }$ while the upper limit is chosen to be 1.

An important observation in the robust $\mathrm{SU}_{\mathrm{Rx}}$ SINR maximisation problem (6.39) is that, at the optimum, either the outage probability constraint or all of the transmit power constraints will be satisfied with equality. This is because both the $\mathrm{SU}_{\mathrm{Rx}}$ SINR and the outage probability are increasing functions of relay transmit power and, therefore, the transmit power will be increased until the outage probability constraint is met with equality or the maximum transmit power budget is 
met, at which point the SINR attains its maximum value in the feasible region. Using this observation, we propose an iterative algorithm (see Algorithm 5), similar to the one described above, that can be used to obtain the optimum solution of the robust $\mathrm{SU}_{\mathrm{Rx}}$ maximisation problem. The algorithm finds the minimum relaxed outage probability specification, $\tilde{\mathrm{P}}_{\mathrm{o}, \max } \geq \mathrm{P}_{\mathrm{o}, \max }$, that either satisfies (6.33) with equality or satisfies (6.12b) $\forall i$ with equality while satisfying (6.33). The bisection technique is used to efficiently search for the minimum relaxed outage probability specification in the interval $\left[\mathrm{P}_{\mathrm{o}, \max }, 1\right]$.

Although Algorithms 4 and 5 provide the optimum beamforming weights, through our extensive numerical simulations we have found that the solutions obtained by directly solving problems (6.38) and (6.39) with the tightened outage probability constraint are very close to the optimum and, in practice, it is not necessary to use the iterative algorithms.

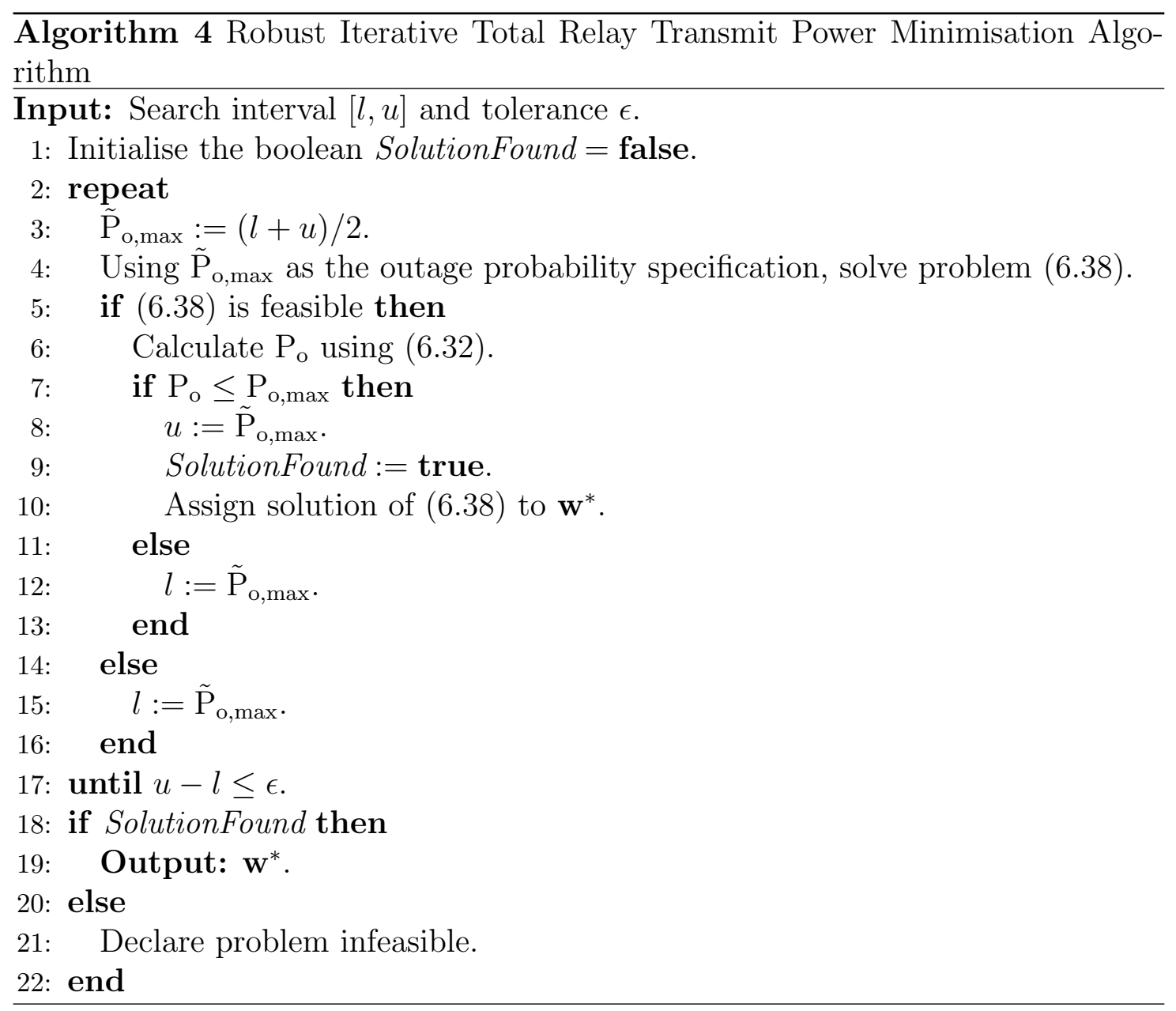




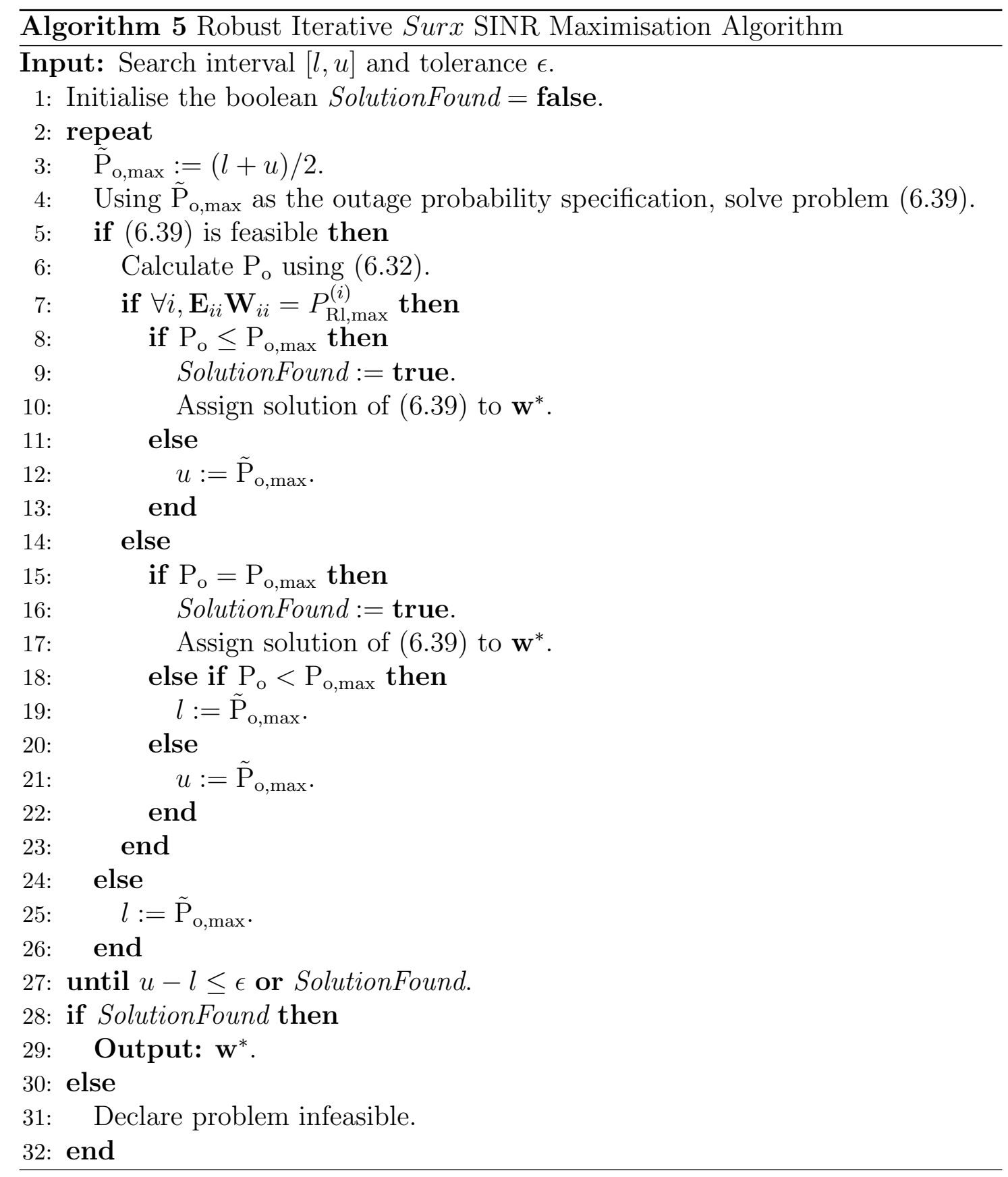




\subsubsection{Partial CSI Availability for the $\mathrm{PU}_{\mathrm{Tx}}$ to $\mathrm{PU}_{\mathrm{Rx}}$ Link and Imperfect CSI Availability for the $\mathrm{SU}_{\mathrm{Rl}}$ to $\mathrm{PU}_{\mathrm{Rx}}$ Links}

In this section, we assume that full CSI is available for all links except for the $\mathrm{PU}_{\mathrm{Tx}}$ to $\mathrm{PU}_{\mathrm{Rx}}$ and $\mathrm{SU}_{\mathrm{Rl}}$ to $\mathrm{PU}_{\mathrm{Rx}}$ links. We assume that only the mean channel power, $\Omega_{\mathrm{pp}}$, of the $\mathrm{PU}_{\mathrm{Tx}}$ to $\mathrm{PU}_{\mathrm{Rx}}$ link is available and that $\mathrm{SU}_{\mathrm{Rl}}$ to $\mathrm{PU}_{\mathrm{Rx}}$ link CSI is imperfect. This imperfection may be due to estimation errors or other factors such as quantisation. Perfect CSI for all other links is available. Our aim is to design a beamformer that is robust against CSI imperfections due to estimation errors for one particular realisation of the $\mathrm{SU}_{\mathrm{Rl}}$ to $\mathrm{PU}_{\mathrm{Rx}}$ channel. The $\mathrm{SU}_{\mathrm{Rl}}$ to $\mathrm{PU}_{\mathrm{Rx}}$ Rayleigh channel, having been instantiated becomes a deterministic unknown. We model this unknown as having non-zero mean, equal to the channel estimate, and small variance, corresponding to the channel uncertainty. (By contrast, in Section 6.4.2 the Rayleigh channel has zero mean, and large variance, equal to the channel power). Adopting the imperfect CSI model of $[274,276]$, we have

$$
\mathbf{h}_{\mathrm{rp}}=\tilde{\mathbf{h}}_{\mathrm{rp}}+\rho \mathbf{e}
$$

where $\tilde{\mathbf{h}}_{\mathrm{rp}}$ is the imperfect $\mathrm{SU}_{\mathrm{Rl}}$ to $\mathrm{PU} \mathrm{Rx}_{\mathrm{Rx}}$ link CSI estimate and $\mathbf{e}$ is the zero-mean estimation error vector with independently distributed complex Gaussian entries and the diagonal covariance matrix $\boldsymbol{\Sigma}_{\mathrm{e}}=\left(\left\|\boldsymbol{\Omega}_{\mathrm{rp}}^{1 / 2}\right\|_{2}^{2} / R\right) \mathbf{I}$, i.e., $\mathbf{e} \sim \mathcal{N}_{\mathcal{C}}\left(\mathbf{0}, \boldsymbol{\Sigma}_{\mathrm{e}}\right)$. We assume that $\tilde{\mathbf{h}}_{\mathrm{rp}}$ is obtained using an unbiased maximum likelihood estimator, hence, over the ensemble of all realisations of the $\mathrm{SU}_{\mathrm{Rl}}$ to $\mathrm{PU} \mathrm{Rx}_{\mathrm{R}}$ channel, $\tilde{\mathbf{h}}_{\mathrm{rp}}$ is a vector with zero-mean complex Gaussian entries and the covariance matrix given by

$$
\xi=\operatorname{diag}\left(\Omega_{\mathrm{rp}}\right)-\rho^{2} \boldsymbol{\Sigma}_{\mathrm{e}}
$$

The $i$ th entry on the main diagonal of $\xi$ is expressed as

$$
\begin{aligned}
\xi_{i i} & =\Omega_{\mathrm{rp}}^{(i)}-\rho^{2} \frac{\left\|\Omega_{\mathrm{rp}}^{1 / 2}\right\|_{2}^{2}}{R} \\
& =\Omega_{\mathrm{rp}}^{(i)}-\frac{\rho^{2}}{R} \sum_{i=1}^{R} \Omega_{\mathrm{rp}}^{(i)} .
\end{aligned}
$$

For the purpose of constructing an optimisation problem, an instance of $\tilde{\mathbf{h}}_{\mathrm{rp}}$ is drawn from the distribution $\mathcal{N}_{\mathcal{C}}(\mathbf{0}, \xi)$ and treated as a deterministic constant. $0 \leq$ $\rho \leq\left(\min \left(\boldsymbol{\Omega}_{\mathrm{rp}}\right) /\left(\left\|\boldsymbol{\Omega}_{\mathrm{rp}}^{1 / 2}\right\|_{2}^{2} / R\right)\right)^{1 / 2}$ determines the quality of the CSI, which is perfect when $\rho=0$ and has maximum uncertainty when $\rho=\left(\min \left(\boldsymbol{\Omega}_{\mathrm{rp}}\right) /\left(\left\|\boldsymbol{\Omega}_{\mathrm{rp}}^{1 / 2}\right\|_{2}^{2} / R\right)\right)^{1 / 2}$. The upper limit on $\rho$ is obtained as follows. Since $\xi$ is a covariance matrix, $\xi_{i i}, \forall i$, 
is greater than or equal to zero. Therefore, we have

$$
\min \left(\boldsymbol{\Omega}_{\mathrm{rp}}\right)-\frac{\rho^{2}}{R} \sum_{i=1}^{R} \Omega_{\mathrm{rp}}^{(i)} \geq 0 .
$$

After rearranging the terms of (6.43), we obtain

$$
\rho \leq \sqrt{\frac{\min \left(\boldsymbol{\Omega}_{\mathrm{rp}}\right)}{\frac{1}{R} \sum_{i=1}^{R} \Omega_{\mathrm{rp}}^{(i)}}}=\sqrt{\frac{\min \left(\boldsymbol{\Omega}_{\mathrm{rp}}\right)}{\frac{1}{R}\left\|\Omega_{\mathrm{rp}}^{1 / 2}\right\|_{2}^{2}}} .
$$

Since $\min \left(\Omega_{\mathrm{rp}}\right) \leq\left\|\Omega_{\mathrm{rp}}^{1 / 2}\right\|_{2}^{2} / R$, the maximum value of $\rho$ for any scenario is 1 , which occurs when all elements of $\Omega_{\mathrm{rp}}$ are equal.

Note that our definition of the error covariance matrix implies that the entries are i.i.d.; however, if the entries have different variances - for instance, the quality of the CSI estimate obtained at each relay node may be different from each other - then the definition can easily be modified to accommodate this without affecting the analysis that follows.

To find an expression for the outage probability (6.27), we first note that using (6.40), $\gamma_{\mathrm{T}} \mathbf{w}^{H}(\mathbf{B}+\mathbf{C}+\mathbf{D}) \mathbf{w}$ can be expressed as

$$
\begin{aligned}
& \gamma_{\mathrm{T}} \mathbf{w}^{H}(\mathbf{B}+\mathbf{C}+\mathbf{D}) \mathbf{w}= \\
& 2 \gamma_{\mathrm{T}} P_{\mathrm{s}} \rho \Re\left\{\mathbf{w}^{H}\left[\mathbf{h}_{\mathrm{sr}} \odot \tilde{\mathbf{h}}_{\mathrm{rp}}\right]\left[\mathbf{h}_{\mathrm{sr}} \odot \mathbf{e}\right]^{H} \mathbf{w}\right\} \\
& +2 \gamma_{\mathrm{T}} P_{\mathrm{p}} \rho \Re\left\{\mathbf{w}^{H}\left[\mathbf{h}_{\mathrm{pr}} \odot \tilde{\mathbf{h}}_{\mathrm{rp}}\right]\left[\mathbf{h}_{\mathrm{pr}} \odot \mathbf{e}\right]^{H} \mathbf{w}\right\} \\
& +2 \gamma_{\mathrm{T}} \sigma_{\mathrm{r}}^{2} \rho \Re\left\{\mathbf{w}^{H} \operatorname{diag}\left(\left(\tilde{\mathbf{h}}_{\mathrm{rp}}^{H}\right)^{T} \odot \mathbf{e}\right) \mathbf{w}\right\} \\
& +\gamma_{\mathrm{T}} P_{\mathrm{s}} \rho^{2} \mathbf{w}^{H}\left[\mathbf{h}_{\mathrm{sr}} \odot \mathbf{e}\right]\left[\mathbf{h}_{\mathrm{sr}} \odot \mathbf{e}\right]^{H} \mathbf{w} \\
& +\gamma_{\mathrm{T}} P_{\mathrm{p}} \rho^{2} \mathbf{w}^{H}\left[\mathbf{h}_{\mathrm{pr}} \odot \mathbf{e}\right]\left[\mathbf{h}_{\mathrm{pr}} \odot \mathbf{e}\right]^{H} \mathbf{w} \\
& +\gamma_{\mathrm{T}} \sigma_{\mathrm{r}}^{2} \rho^{2} \mathbf{w}^{H} \operatorname{diag}\left(|\mathbf{e}|^{2}\right) \mathbf{w} \\
& +\gamma_{\mathrm{T}} \mathbf{w}^{H}\left[P_{\mathrm{s}}\left[\mathbf{h}_{\mathrm{sr}} \odot \tilde{\mathbf{h}}_{\mathrm{rp}}\right]\left[\mathbf{h}_{\mathrm{sr}} \odot \tilde{\mathbf{h}}_{\mathrm{rp}}\right]^{H}\right. \\
& \left.\quad+P_{\mathrm{p}}\left[\mathbf{h}_{\mathrm{pr}} \odot \tilde{\mathbf{h}}_{\mathrm{rp}}\right]\left[\mathbf{h}_{\mathrm{pr}} \odot \tilde{\mathbf{h}}_{\mathrm{rp}}\right]^{H}+\sigma_{\mathrm{r}}^{2} \operatorname{diag}\left(\left|\tilde{\mathbf{h}}_{\mathrm{rp}}\right|^{2}\right)\right] \mathbf{w}
\end{aligned}
$$

The terms on the right hand side of (6.45) are denoted by $r_{1}, r_{2}, \ldots, r_{7}$, PDFs of which are given by Lemma 6.4.3. We first present Lemma 6.4.2 which is useful in deriving the PDFs of some of the terms of (6.45).

Lemma 6.4.2 If $\mathbf{x} \in \mathcal{C}^{R \times 1}$ is a zero-mean random vector with complex Gaussian elements and the covariance matrix $\mathbf{\Sigma}$, i.e. $\mathbf{x} \sim \mathcal{N}_{\mathcal{C}}(\mathbf{0}, \mathbf{\Sigma})$, then for any deterministic vectors, $\mathbf{u}, \mathbf{g} \in \mathcal{C}^{R \times 1}$, the random variable $\psi=\mathbf{g}^{H} \mathbf{u x}{ }^{H} \mathbf{g}$ is a zero-mean complex Gaussian random variable with variance

$$
\sigma_{\psi}^{2}=\operatorname{tr}(\boldsymbol{\Sigma} \mathbf{G}) \operatorname{tr}(\mathbf{U G}),
$$


where $\mathbf{G}=\mathbf{g g}^{H}$ and $\mathbf{U}=\mathbf{u u}^{H}$.

\section{Proof}

Since the entries of $\mathbf{x}$ are zero-mean complex Gaussian random variables and $\psi$ is a linear combination of the entries of $\mathbf{x}$, it implies that $\psi$ is also a zero-mean complex Gaussian random variable. Note that $\mathbf{g}^{H} \mathbf{u x} \mathbf{x}^{H} \mathbf{g}=\mathbf{x}^{H} \mathbf{g g}^{H} \mathbf{u}$ and therefore the variance of $\psi$ is given by

$$
\begin{aligned}
\sigma_{\psi}^{2} & =\mathbb{E}\left\{\mathbf{u}^{H} \mathbf{g g}{ }^{H} \mathbf{x} \mathbf{x}^{H} \mathbf{g g}^{H} \mathbf{u}\right\} \\
& =\mathbf{u}^{H} \mathbf{g g}^{H} \mathbb{E}\left\{\mathbf{x} \mathbf{x}^{H}\right\} \mathbf{g g}^{H} \mathbf{u} \\
& =\mathbf{u}^{H} \mathbf{g} \operatorname{tr}(\boldsymbol{\Sigma} \mathbf{G}) \mathbf{g}^{H} \mathbf{u} \\
& =\operatorname{tr}(\boldsymbol{\Sigma} \mathbf{G}) \mathbf{u}^{H} \mathbf{g g} \mathbf{g}^{H} \mathbf{u} \\
& =\operatorname{tr}(\boldsymbol{\Sigma} \mathbf{G}) \operatorname{tr}(\mathbf{U} \mathbf{G}) .
\end{aligned}
$$

Lemma 6.4.3 $r_{1}, r_{2}$ and $r_{3}$ are zero-mean Gaussian random variables with variances, $\sigma_{1}^{2}, \sigma_{2}^{2}$ and $\sigma_{1}^{3}$ given by

$$
\begin{array}{r}
\sigma_{1}^{2}=2 \gamma_{\mathrm{T}}^{2} P_{\mathrm{s}}^{2} \operatorname{tr}\left(\left(\mathbf{h}_{\mathrm{sr}} \mathbf{h}_{\mathrm{sr}}^{H} \odot \rho^{2} \boldsymbol{\Sigma}_{\mathrm{e}}\right) \mathbf{W}\right) \\
\operatorname{tr}\left(\left[\mathbf{h}_{\mathrm{sr}} \odot \tilde{\mathbf{h}}_{\mathrm{rp}}\right]\left[\mathbf{h}_{\mathrm{sr}} \odot \tilde{\mathbf{h}}_{\mathrm{rp}}\right]^{H} \mathbf{W}\right) \\
\sigma_{2}^{2}=2 \gamma_{\mathrm{T}}^{2} P_{\mathrm{p}}^{2} \operatorname{tr}\left(\left(\mathbf{h}_{\mathrm{pr}} \mathbf{h}_{\mathrm{pr}}^{H} \odot \rho^{2} \boldsymbol{\Sigma}_{\mathrm{e}}\right) \mathbf{W}\right) \\
\operatorname{tr}\left(\left[\mathbf{h}_{\mathrm{pr}} \odot \tilde{\mathbf{h}}_{\mathrm{rp}}\right]\left[\mathbf{h}_{\mathrm{pr}} \odot \tilde{\mathbf{h}}_{\mathrm{rp}}\right]^{H} \mathbf{W}\right), \\
\sigma_{3}^{2}=2 \gamma_{\mathrm{T}}^{2} \sigma_{\mathrm{r}}^{4}\left(\operatorname{vec}\left(\mathbf{W}^{H}\right)\right)^{H} \boldsymbol{\Sigma}_{\tilde{E}} \operatorname{vec}\left(\mathbf{W}^{H}\right),
\end{array}
$$

where $\mathbf{W}=\mathbf{w} \mathbf{w}^{H}$ and $\boldsymbol{\Sigma}_{\tilde{E}}=\mathbb{E}\left\{\operatorname{vec}(\tilde{\mathbf{E}}) \operatorname{vec}(\tilde{\mathbf{E}})^{H}\right\}$ is an $R^{2} \times R^{2}$ diagonal matrix with entries on the main diagonal given by, $\boldsymbol{\Sigma}_{\tilde{E}_{j j}}=\rho^{2} \boldsymbol{\Sigma}_{\mathrm{e}_{i i}}\left|\tilde{h}_{\mathrm{rp}}^{(i)}\right|^{2}, i=1 \ldots R, j=$ $i(R+1)-R$, and zeros everywhere else.

$r_{4}$ and $r_{5}$ are exponentially distributed random variables with means, $\mu_{4}$ and $\mu_{5}$ given by

$$
\begin{aligned}
& \mu_{4}=\gamma_{\mathrm{T}} P_{\mathrm{s}} \operatorname{tr}\left(\left(\mathbf{h}_{\mathrm{sr}} \mathbf{h}_{\mathrm{sr}}^{H} \odot \rho^{2} \boldsymbol{\Sigma}_{\mathrm{e}}\right) \mathbf{W}\right), \\
& \mu_{5}=\gamma_{\mathrm{T}} P_{\mathrm{p}} \operatorname{tr}\left(\left(\mathbf{h}_{\mathrm{pr}} \mathbf{h}_{\mathrm{pr}}^{H} \odot \rho^{2} \boldsymbol{\Sigma}_{\mathrm{e}}\right) \mathbf{W}\right) .
\end{aligned}
$$

$r_{6}$ is a sum of $R$ independent exponentially distributed random variables with rate parameters $\lambda_{i}=1 /\left(\gamma_{\mathrm{T}} \sigma_{\mathrm{r}}^{2} \rho^{2} \Sigma_{\mathrm{e}_{i i}} \mathbf{W}_{i i}\right), i=1 \ldots R$ and the mean and variance, $\mu_{6}$ and $\sigma_{6}^{2}$, respectively, given by

$$
\begin{aligned}
\mu_{6} & =\left[\prod_{i=1}^{R} \lambda_{i}\right] \sum_{j=1}^{R} \frac{1}{\lambda_{j}^{2} \prod_{k=1, k \neq j}^{N}\left(\lambda_{k}-\lambda_{j}\right)}, \\
\sigma_{6}^{2} & =\left[\prod_{i=1}^{R} \lambda_{i}\right] \sum_{j=1}^{R} \frac{2}{\lambda_{j}^{3} \prod_{k=1, k \neq j}^{N}\left(\lambda_{k}-\lambda_{j}\right)}-\mu_{6}^{2} .
\end{aligned}
$$


$r_{7}$ is a deterministic constant.

\section{Proof}

Using Lemma 6.4.2, we see that $r_{1}$ and $r_{2}$ are zero-mean Gaussian random variables with variances given by (6.47) and (6.48), respectively.

Since $r_{3}$ is a linear combination of zero-mean Gaussian random variables, it is also a zero-mean Gaussian random variable. By defining $\tilde{\mathbf{E}}=\rho \operatorname{diag}\left(\left(\tilde{\mathbf{h}}_{\mathrm{rp}}^{H}\right)^{T} \odot \mathbf{e}\right)$, the variance can be expressed as

$$
\sigma_{3}^{2}=2 \gamma_{\mathrm{T}}^{2} \sigma_{\mathrm{r}}^{4} \mathbb{E}\left\{\operatorname{tr}(\mathbf{W} \tilde{\mathbf{E}}) \operatorname{tr}(\mathbf{W} \tilde{\mathbf{E}})^{*}\right\}
$$

Invoking [95, Theorem 1.2.22. (ii)], which states that

$$
\operatorname{tr}(\mathbf{W} \tilde{\mathbf{E}})=\left(\operatorname{vec}\left(\mathbf{W}^{H}\right)\right)^{H} \operatorname{vec}(\tilde{\mathbf{E}}),
$$

and because $\boldsymbol{\Sigma}_{\mathrm{e}}$ is a diagonal matrix, (6.54) can be rewritten as (6.49).

Using Lemma 6.4.1, $r_{4}$ and $r_{5}$ are recognised as exponentially distributed random variables with means given by (6.50) and (6.51), respectively.

It is easy to show that $r_{6}$ can be expressed as

$$
r_{6}=\gamma_{\mathrm{T}} \sigma_{\mathrm{r}}^{2} \rho^{2} \sum_{i=1}^{R}\left|w_{i}\right|^{2}\left|e_{i}\right|^{2} .
$$

Since the entries of e are independently distributed Gaussian random variables, $\left|e_{i}\right|^{2} \forall i$, are independently distributed exponential random variables and therefore, (6.55) is a sum of $R$ independent exponentially distributed random variables whose mean and variance is known to have the forms given by (6.52) and (6.53), respectively.

Since the expression of $r_{7}$ does not contain any random variables, it is a deterministic constant. This completes the proof .

Due to the correlation between the terms of (6.45), its exact PDF is difficult to handle. However, we propose an accurate approximation of the PDF which is easier to handle based on the following observation. In a practical cognitive radio system, the PU requires a very reliable link, hence the outage probability specified will generally be very small. In order to satisfy the stringent outage probability constraint, both $\sigma_{1}^{2}$ and $\sigma_{2}^{2}$ must also be small. Notice that the expression for $\sigma_{1}^{2}$ contains the term $P_{\mathrm{s}} \operatorname{tr}\left(\left(\mathbf{h}_{\mathrm{sr}} \mathbf{h}_{\mathrm{sr}}^{H} \odot \rho^{2} \boldsymbol{\Sigma}_{\mathrm{e}}\right) \mathbf{W}\right)$, which can be rewritten as $P_{\mathrm{s}} \sum_{i=1}^{R} \rho^{2} \boldsymbol{\Sigma}_{\mathrm{e}_{i i}}\left|h_{\mathrm{sr}}^{(i)}\right|^{2} \mathbf{W}_{i i}$. This term represents the $\mathrm{SU}$ interference that is generated at the $\mathrm{PU}_{\mathrm{Rx}}$ due to CSI errors, and its level can only be controlled by adjusting the beamformer transmit power. Hence, as the $\mathrm{SU}_{\mathrm{Tx}}$ to $\mathrm{SU}_{\mathrm{Rl}}$ link gets stronger, the beamformer weights will be scaled down in order to achieve the outage probability constraint. Note that this term also appears in $\mu_{4}$, which is used in our final approximation, (6.63), of the 
PU outage probability constraint and its magnitude is controlled by controlling the magnitude of $\mu_{4}$. We note that the beamformer is able to control interference from the $P_{\mathrm{s}} \operatorname{tr}\left(\left[\mathbf{h}_{\mathrm{sr}} \odot \tilde{\mathbf{h}}_{\mathrm{rp}}\right]\left[\mathbf{h}_{\mathrm{sr}} \odot \tilde{\mathbf{h}}_{\mathrm{rp}}\right]^{H} \mathbf{W}\right)$ part of $\sigma_{1}^{2}$ through both amplitude and phase control and is able to keep it sufficiently low to satisfy the outage probability constraint. Again, note that this term appears in the deterministic constant $r_{7}$, which is used in (6.63). Hence, the magnitude of this term is controlled by controlling the magnitude of $r_{7}$.

In the $\mathrm{SU}_{\mathrm{Rx}} \mathrm{SINR}$ maximisation problem (6.12), the individual relay transmit power constraints also limit the beamformer weight magnitudes, which in turn limit the levels of $\sigma_{1}^{2}$ and $\sigma_{2}^{2}$. From the definition of $\mathbf{E}$ and $(6.12 \mathrm{~b})$, we see that for a fixed value of $P_{\mathrm{Rl}, \max }^{(i)}$, the $i$ th relay's maximum beamformer weight magnitude achievable decreases as either the $\mathrm{SU}_{\mathrm{Tx}}$ or $\mathrm{PU}_{\mathrm{Tx}}$ to the $i$ th relay link gets stronger.

The expression for $\sigma_{2}^{2}$ contains two terms that represent PU self interference the level of which is controlled in a similar way to that described above, i.e., by controlling the levels of $\mu_{5}$ and $r_{7}$, both of which appear in (6.63). Since both $\sigma_{1}^{2}$ and $\sigma_{2}^{2}$ are expected to be small, the PDF of $r_{1}$ and $r_{2}$ will be concentrated around zero and can be neglected.

Note that $\sigma_{\mathrm{r}}^{2}$ is generally small — for instance, a receiver with a $2 \mathrm{MHz}$ bandwidth and a noise figure (NF) of $30 \mathrm{~dB}$ operating at a room temperature of 293 $\mathrm{K}$ has an effective noise power of approximately $-80 \mathrm{dBm}-\sigma_{3}^{2}$ is very small and therefore, the PDF of $r_{3}$ is concentrated around zero and can be safely ignored. Similarly, both $\mu_{6}$ and $\sigma_{6}^{2}$ are very small and the PDF of $r_{6}$ is also concentrated near zero and can be neglected.

From the above discussion, we see that the PDF of (6.45) can be approximated as the sum of two correlated exponentially distributed random variables $r_{4}$ and $r_{5}$. Next, we show that the correlation between $r_{4}$ and $r_{5}$ is small and therefore they can be treated as independent random variables. By letting $\mathbf{H}_{1}=\mathbf{h}_{\mathrm{sr}} \mathbf{h}_{\mathrm{sr}}^{H} \odot \rho^{2} \mathbf{e} \mathrm{e}^{H}$ and $\mathbf{H}_{2}=\mathbf{h}_{\mathrm{pr}} \mathbf{h}_{\mathrm{pr}}^{H} \odot \rho^{2} \mathbf{e e}^{H}$, the covariance between $r_{4}$ and $r_{5}$ is given by

$$
\begin{aligned}
\operatorname{Cov}\left(r_{4}, r_{5}\right) & =\gamma_{\mathrm{T}}^{2} P_{\mathrm{s}}^{2} \mathbb{E}\left\{\operatorname{tr}\left(\mathbf{W H}_{1}\right) \operatorname{tr}\left(\mathbf{W H}_{2}\right)^{*}\right\}-\mu_{4} \mu_{5} \\
& =\gamma_{\mathrm{T}}^{2} P_{\mathrm{s}}^{2} \operatorname{vec}\left(\mathbf{W}^{H}\right)^{H} \mathbb{E}\left\{\operatorname{vec}\left(\mathbf{H}_{1}\right) \operatorname{vec}\left(\mathbf{H}_{2}\right)^{H}\right\} \operatorname{vec}\left(\mathbf{W}^{H}\right)-\mu_{4} \mu_{5} \\
& =\gamma_{\mathrm{T}}^{2} P_{\mathrm{s}}^{2} \sum_{i=1}^{R} \sum_{j=1}^{R}\left(\mathbf{h}_{\mathrm{sr}} \mathbf{h}_{\mathrm{sr}}^{H}\right)_{i j}\left(\mathbf{h}_{\mathrm{pr}} \mathbf{h}_{\mathrm{pr}}^{H}\right)_{i j}^{*} \rho^{4} \boldsymbol{\Sigma}_{\mathrm{e}_{i i}} \boldsymbol{\Sigma}_{\mathrm{e}_{j j}}\left|\mathbf{W}_{i j}\right|^{2}
\end{aligned}
$$

It is evident from (6.56) that for small values of $\rho \Sigma_{\mathrm{e}_{i i}}, \forall i$, the covariance is low. Recall that when the $\mathrm{SU}_{\mathrm{Tx}}$ to $\mathrm{SU}_{\mathrm{Rl}}$ and $\mathrm{PU}_{\mathrm{Tx}}$ to $\mathrm{SU}_{\mathrm{Rl}}$ links are strong, the beamformer weights are scaled down to meet the outage probability constraint. In this scenario, $\left|\mathbf{W}_{i j}\right|^{2}, \forall i, j$ will be small and the covariance will tend to be low. Therefore, in our analysis, we treat $r_{4}$ and $r_{5}$ as independent random variables. 
Hence, $\gamma_{\mathrm{T}} \mathbf{w}^{H}(\mathbf{B}+\mathbf{C}+\mathbf{D}) \mathbf{w}$ can be approximated as

$$
\gamma_{\mathrm{T}} \mathbf{w}^{H}(\mathbf{B}+\mathbf{C}+\mathbf{D}) \mathbf{w} \approx r_{4}+r_{5}+r_{7}
$$

and the outage probability can be approximated by

$$
\mathrm{P}_{\mathrm{o}} \approx \operatorname{Pr}\left\{P_{\mathrm{p}}\left|h_{\mathrm{pp}}\right|^{2}-\left(r_{4}+r_{5}\right) \leq \gamma_{\mathrm{T}} \sigma_{\mathrm{p}}^{2}+r_{7}\right\}
$$

Note that the PDF in (6.58) is the difference between an exponentially distributed random variable and the sum of two independent exponentially distributed random variables. In Fig. 6.2, we show a comparison of the empirical CDF obtained through Monte Carlo simulations and the approximation (6.58) for $\rho=0.5$ in three channel conditions where the signal to interference channel power ratios (SICR) are set to $8 \mathrm{~dB}, 3 \mathrm{~dB}$ and $0.8 \mathrm{~dB}$, i.e., $\Omega_{\mathrm{sr}}^{(i)} / \Omega_{\mathrm{pr}}=\Omega_{\mathrm{rs}}^{(i)} / \Omega_{\mathrm{ps}}=\Omega_{\mathrm{pp}} / \Omega_{\mathrm{rp}}^{(i)}=\{8,3,0.8\} \mathrm{dB}$ $\forall i$. In all three cases, there are 8 relay nodes, $P_{\mathrm{p}}=P_{\mathrm{s}}=30 \mathrm{dBm}, P_{\mathrm{Rl}, \max }^{(i)}=30$ $\mathrm{dBm} \forall i, \gamma_{\mathrm{T}}=5 \mathrm{~dB}, \gamma_{\mathrm{s}, \min }=0 \mathrm{~dB}$, noise power at each receiver is assumed to be $-80 \mathrm{dBm}$, the maximum $\mathrm{PU}_{\mathrm{Rx}}$ outage probability, $\mathrm{P}_{\mathrm{o}, \max }$, is set to $5 \%$ and $\boldsymbol{\Sigma}_{\mathrm{e}_{i i}}=\left\|\boldsymbol{\Omega}_{\mathrm{rp}}^{1 / 2}\right\|_{2}^{2} / 8, \forall i$. Due to space constraints, the empirical and approximated CDF for each channel condition is shown only for one realisation of the channel vectors, where the vectors have been scaled to obtain the required SICR. However, the approximation holds for any realisation of the channel vectors, since no assumptions have been made about channel vectors in its derivation. The empirical and approximated CDF for each channel condition is obtained by first designing a robust beamformer for $\mathrm{SU}_{\mathrm{Rx}} \mathrm{SINR}$ maximisation problem (6.65) and then using the resulting beamformer in Monte Carlo simulations and in the analytical expression for the approximation. It is evident that the approximation accurately represents the empirical CDF. Similar results are obtained for the robust $\mathrm{SU}_{\mathrm{Rl}}$ transmit power minimisation problem (6.64).

Using the approximation in (6.58), the outage probability is expressed as

$$
\mathrm{P}_{\mathrm{o}}=1-\exp \left(-\frac{\gamma_{\mathrm{T}} \sigma_{\mathrm{p}}^{2}+r_{7}}{P_{\mathrm{p}} \Omega_{\mathrm{pp}}}\right)\left(\frac{1}{1+\frac{\mu_{4}}{P_{\mathrm{p}} \Omega_{\mathrm{pp}}}}\right)\left(\frac{1}{1+\frac{\mu_{5}}{P_{\mathrm{p}} \Omega_{\mathrm{pp}}}}\right),
$$

and the outage probability constraint is given by

$$
\exp \left(\frac{r_{7}}{P_{\mathrm{p}} \Omega_{\mathrm{pp}}}\right)\left(1+\frac{\mu_{4}}{P_{\mathrm{p}} \Omega_{\mathrm{pp}}}\right)\left(1+\frac{\mu_{5}}{P_{\mathrm{p}} \Omega_{\mathrm{pp}}}\right) \leq \frac{\exp \left(-\frac{\gamma_{\mathrm{T}} \sigma_{\mathrm{p}}^{2}}{P_{\mathrm{p}} \Omega_{\mathrm{pp}}}\right)}{1-\mathrm{P}_{\mathrm{o}, \mathrm{max}}}
$$

It is worth noting that, when there are no $\mathrm{SU}_{\mathrm{Rl}}$ to $\mathrm{PU}_{\mathrm{Rx}}$ link CSI errors, constraint (6.60) reduces to constraint (6.23). This is expected since the only channel uncertainty remaining is in the $\mathrm{PU}_{\mathrm{Tx}}$ to $\mathrm{PU}_{\mathrm{Rx}}$ link, which was analysed in Sec- 


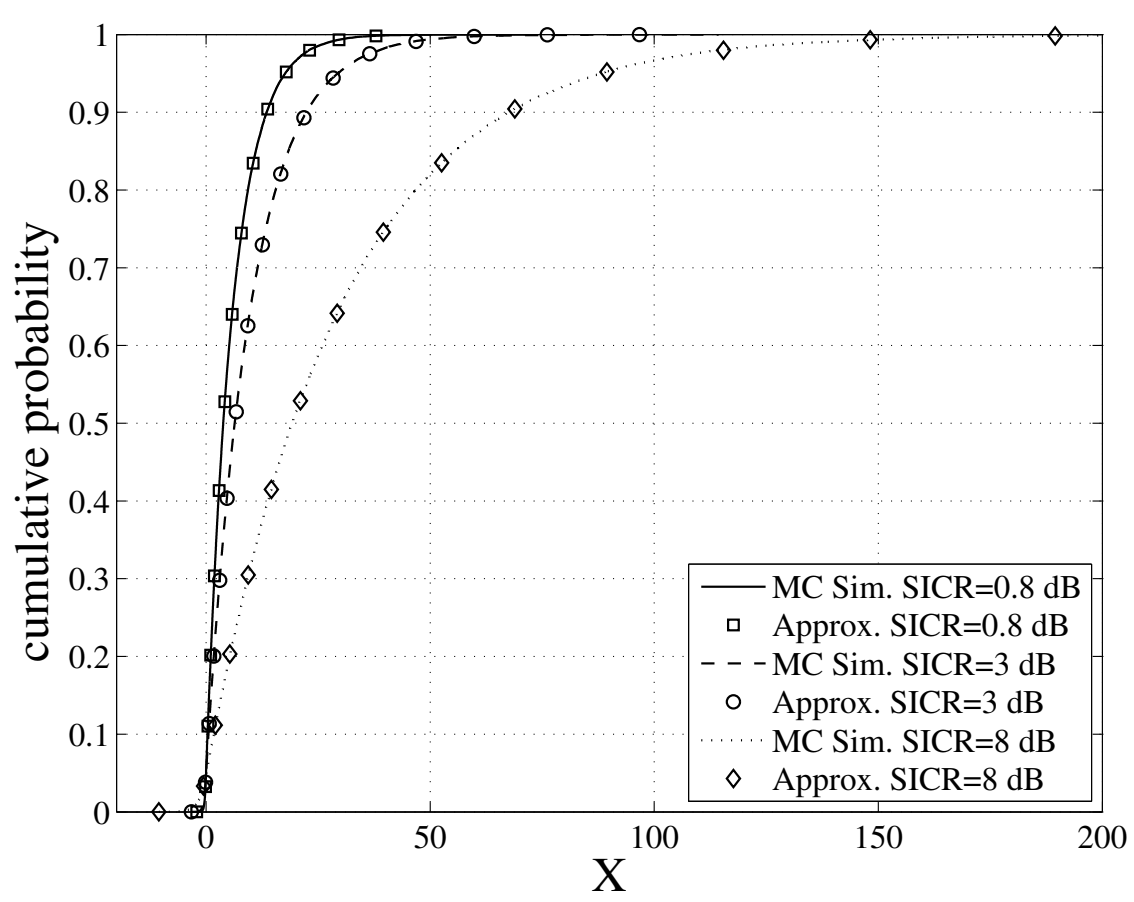

Figure 6.2: Empirical and Approximated CDF of (6.58).

tion 6.4.1.

We use the geometric-arithmetic mean inequality and rewrite (6.60) as

$$
\exp \left(\frac{r_{7}}{P_{\mathrm{p}} \Omega_{\mathrm{pp}}}\right)+\left(1+\frac{\mu_{4}}{P_{\mathrm{p}} \Omega_{\mathrm{pp}}}\right)+\left(1+\frac{\mu_{5}}{P_{\mathrm{p}} \Omega_{\mathrm{pp}}}\right) \leq 3\left(\frac{\exp \left(-\frac{\gamma_{\mathrm{T}} \sigma_{\mathrm{p}}^{2}}{P_{\mathrm{p}} \Omega_{\mathrm{pp}}}\right)}{1-\mathrm{P}_{\mathrm{o}, \max }}\right)^{\frac{1}{3}}
$$

Note that (6.61) is a non-convex constraint and is difficult to handle. However, the assumptions that were made to obtain the approximate outage probability expression also imply that $r_{7}$ is small. Thus, $\exp \left(r_{7} /\left(P_{\mathrm{p}} \Omega_{\mathrm{pp}}\right)\right) \approx\left(1+r_{7} /\left(P_{\mathrm{p}} \Omega_{\mathrm{pp}}\right)\right)$, allowing us to write the outage probability constraint as the convex constraint

$$
\left.\frac{1}{P_{\mathrm{p}} \Omega_{\mathrm{pp}}}\left(r_{7}+\mu_{4}+\mu_{5}\right)+3\left(1-\left(\frac{\exp \left(-\frac{\gamma_{\mathrm{T}} \sigma_{\mathrm{p}}^{2}}{P_{\mathrm{p}} \Omega_{\mathrm{pp}}}\right)}{1-\mathrm{P}_{\mathrm{o}, \max }}\right)\right)^{\frac{1}{3}}\right) 0 .
$$

or equivalently as the SOCP

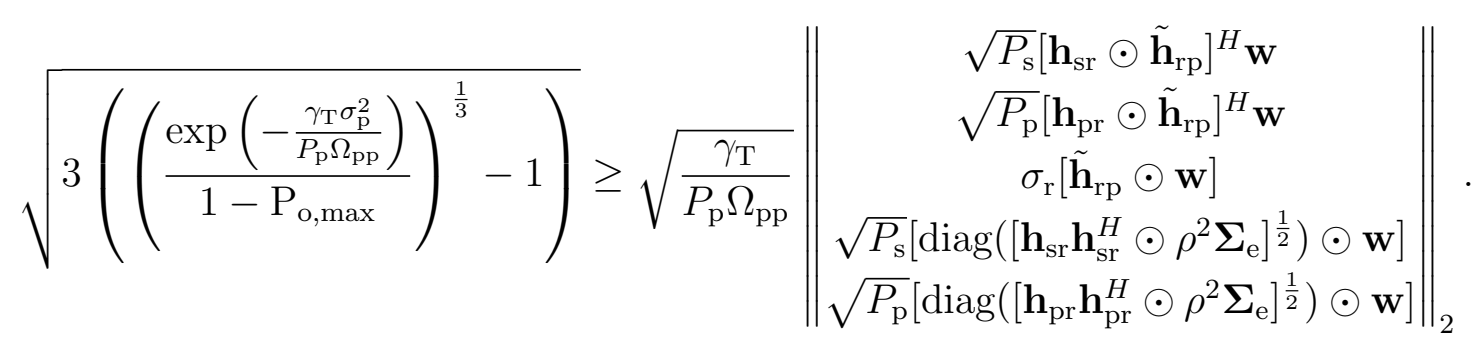


The robust $\mathrm{SU}_{\mathrm{R}}$ power minimisation problem with the tightened outage probability SOCP constraint can therefore be expressed as

$$
\min _{\mathbf{w}} \mathbf{w}^{H} \mathbf{E w}, \quad \text { s.t. } \quad(6.63) \text { and }(6.11 \mathrm{c})
$$

The robust $\mathrm{SU}_{\mathrm{Rx}} \mathrm{SINR}$ maximisation problem can be expressed as

$$
\begin{array}{ll}
\max _{\mathbf{W}} & \frac{\operatorname{tr}(\mathbf{Q W})}{\operatorname{tr}((\mathbf{R}+\mathbf{V}) \mathbf{W})+P_{\mathrm{p}}\left|h_{\mathrm{ps}}\right|^{2}+\sigma_{\mathrm{s}}^{2}} \\
\text { s.t. } & (6.16 \mathrm{~b}) \text { and }(6.16 \mathrm{~d}) \\
& \frac{1}{P_{\mathrm{p}} \Omega_{\mathrm{pp}}}\left(\tilde{r}_{7}+\mu_{4}+\mu_{5}\right) \leq 3\left(\left(\frac{\exp \left(-\frac{\gamma_{\mathrm{T}} \sigma_{\mathrm{p}}^{2}}{P_{\mathrm{p}} \Omega_{\mathrm{pp}}}\right)}{1-\mathrm{P}_{\mathrm{o}, \max }}\right)^{\frac{1}{3}}-1\right),
\end{array}
$$

where

$$
\begin{aligned}
\tilde{r}_{7} \triangleq \gamma_{\mathrm{T}} \operatorname{tr} & \left(\left(P_{\mathrm{s}}\left[\mathbf{h}_{\mathrm{sr}} \odot \tilde{\mathbf{h}}_{\mathrm{rp}}\right]\left[\mathbf{h}_{\mathrm{sr}} \odot \tilde{\mathbf{h}}_{\mathrm{rp}}\right]^{H}+\sigma_{\mathrm{r}}^{2} \operatorname{diag}\left(\left|\tilde{\mathbf{h}}_{\mathrm{rp}}\right|^{2}\right)\right.\right. \\
& \left.\left.+P_{\mathrm{p}}\left[\mathbf{h}_{\mathrm{pr}} \odot \tilde{\mathbf{h}}_{\mathrm{rp}}\right]\left[\mathbf{h}_{\mathrm{pr}} \odot \tilde{\mathbf{h}}_{\mathrm{rp}}\right]^{H}\right) \mathbf{W}\right) .
\end{aligned}
$$

Problem (6.65) can be solved using the method described in Section 6.3.2.

Since problems (6.64) and (6.65) have the same form as (6.38) and (6.39), respectively, the iterative algorithms proposed in Section 6.4 .2 can be used to improve on the solutions obtained by solving (6.64) and (6.65). However, through our extensive numerical simulations, we have found that the improvements are marginal and do not motivate the use of the iterative algorithms.

\subsection{Simulation Results and Discussion}

We illustrate the performance of our proposed methods through numerical simulations in i.i.d. Rayleigh flat-fading channels. We consider a system with 8 relay nodes. In all simulations we have set $P_{\mathrm{p}}=P_{\mathrm{s}}=30 \mathrm{dBm}, P_{\mathrm{Rl} \text {,max }}^{(i)}=30 \mathrm{dBm} \forall i$, $\gamma_{\mathrm{T}}=5 \mathrm{~dB}$ and the noise power at each receiver is assumed to be $-80 \mathrm{dBm}$, i.e., $\sigma_{\mathrm{p}}^{2}=\sigma_{\mathrm{r}}^{2}=\sigma_{\mathrm{s}}^{2}=-80 \mathrm{dBm}$. The maximum $\mathrm{PU}_{\mathrm{Rx}}$ outage probability, $\mathrm{P}_{\mathrm{o}, \max }$, is set to $5 \%$. Channel powers of the direct paths, i.e., $\Omega_{\mathrm{pp}}, \Omega_{\mathrm{sr}}^{(i)} \forall i$ and $\Omega_{\mathrm{rs}}^{(i)} \forall i$, are set to $10 \mathrm{~dB}$. For our simulations we have set the SICR of all receivers to $5 \mathrm{~dB}$. Simulations for the total relay power minimisation problem have $\gamma_{\mathrm{s}, \min }=0 \mathrm{~dB}$. According to CSI error model (6.40), $\boldsymbol{\Sigma}_{\mathrm{e}_{i i}}=\left\|\boldsymbol{\Omega}_{\mathrm{rp}}^{1 / 2}\right\|_{2}^{2} / 8=5 \mathrm{~dB}, \forall i$. To illustrate the impact of CSI errors and the effectiveness of our proposed method, we present simulation results for four different values of $\rho$, namely, $0.05,0.2,0.3$ and 0.5 .

The results obtained from our methods are compared against the full CSI, worstcase and non-robust designs. As the name suggests, the worst-case beamformer guarantees that the SINR at the $\mathrm{PU}_{\mathrm{Rx}}$ is above the threshold $\gamma_{\mathrm{T}}$ in the worst-case 
channel condition. Since an instantaneous realisation of $h_{\mathrm{pp}}$ is not available for the beamformer design of Section 6.4.1, our worst-case design solves problems (6.8) and (6.12) based on the expected value of (6.7). Note that (6.7) is at its minimum when $\left|h_{\mathrm{pp}}\right|^{2}=\Omega_{\mathrm{pp}}-\epsilon_{1}$ for some appropriately chosen value of $\epsilon_{1} \geq 0$. The worst-case beamformer ensures that this minimum value is always above the threshold $\gamma_{\mathrm{T}}$. To provide a fair comparison with the methods proposed in this paper, $\epsilon_{1}$ is chosen such that $\operatorname{Pr}\left\{\left|h_{\mathrm{pp}}\right|^{2} \geq \Omega_{\mathrm{pp}}-\epsilon_{1}\right\}=1-\mathrm{P}_{\mathrm{o}, \max }$. Similarly, the expected value of (6.7) is used to design the worst-case beamformer of Section 6.4.2 since instantaneous realisations of both $h_{\mathrm{pp}}$ and $\mathbf{h}_{\mathrm{rp}}$ are not available. In this case the expected value of (6.7) is at its minimum when $\left|h_{\mathrm{pp}}\right|^{2}=\Omega_{\mathrm{pp}}-\epsilon_{1}$ and $\left|h_{\mathrm{rp}}^{(i)}\right|^{2}=\Omega_{\mathrm{rp}}^{(i)}+\epsilon_{2} \forall i$, for some appropriately chosen values of $\epsilon_{1}, \epsilon_{2} \geq 0$. $\epsilon_{1}$ and $\epsilon_{2}$ are chosen such that $\operatorname{Pr}\left\{\left|h_{\mathrm{pp}}\right|^{2} \geq \Omega_{\mathrm{pp}}-\epsilon_{1}\right\} \prod_{i=1}^{R} \operatorname{Pr}\left\{\left|h_{\mathrm{rp}}^{(i)}\right|^{2} \leq \Omega_{\mathrm{rp}}^{(i)}+\epsilon_{2}\right\}=1-\mathrm{P}_{\mathrm{o}, \max }$.

To derive the worst-case beamformer of Section 6.4.3, we use channel uncertainty model (6.40), with $\rho=1$. Here, e is the error vector which has a norm bound of $\epsilon_{3}$, i.e., $\|\mathbf{e}\|_{2} \leq \epsilon_{3}$. The worst-case beamformer will ensure that the $\mathrm{PU}_{\mathrm{Rx}} \mathrm{SINR}$ is always above $\gamma_{\mathrm{T}}$ for all CSI error vectors satisfying $\|\mathbf{e}\|_{2} \leq \epsilon_{3}$ and $\left|h_{\mathrm{pp}}\right|^{2} \geq \Omega_{\mathrm{pp}}-\epsilon_{1}$. Using (6.40) and the worst-case value of $\left|h_{\mathrm{pp}}\right|^{2}$ in (6.7), the $\mathrm{PU}_{\mathrm{Rx}}$ SINR constraint can be expressed as

$$
\begin{aligned}
& -\tilde{\mathbf{h}}_{\mathrm{rp}}^{H} \mathbf{F} \tilde{\mathbf{h}}_{\mathrm{rp}}-\tilde{\mathbf{h}}_{\mathrm{rp}}^{H} \mathbf{F e}-\mathbf{e}^{H} \mathbf{F} \tilde{\mathbf{h}}_{\mathrm{rp}}-\mathbf{e}^{H} \mathbf{F e} \\
& -\sigma_{\mathrm{p}}^{2}+\frac{P_{\mathrm{p}}\left(\Omega_{\mathrm{pp}}-\epsilon_{1}\right)}{\gamma_{\mathrm{T}}} \geq 0, \\
& \text { s.t. } \quad 1-\left\|\frac{\mathbf{e}}{\epsilon_{3}}\right\|_{2}^{2} \geq 0
\end{aligned}
$$

where $\mathbf{F}=P_{\mathrm{s}}\left(\mathbf{h}_{\mathrm{sr}} \mathbf{h}_{\mathrm{sr}}^{H} \odot \mathbf{W}\right)+P_{\mathrm{p}}\left(\mathbf{h}_{\mathrm{pr}} \mathbf{h}_{\mathrm{pr}}^{H} \odot \mathbf{W}\right)+\sigma_{\mathrm{r}}^{2}(\mathbf{I} \odot \mathbf{W})$. The S-Procedure, which was introduced in Section 3.1.2, can be used to combine the two constraints in (6.66) into one convex constraint. The S-Procedure states that

$$
\begin{array}{r}
\exists_{s \geq 0} \mid-\tilde{\mathbf{h}}_{\mathrm{rp}}^{H} \mathbf{F} \tilde{\mathbf{h}}_{\mathrm{rp}}-\tilde{\mathbf{h}}_{\mathrm{rp}}^{H} \mathbf{F e}-\mathbf{e}^{H} \mathbf{F} \tilde{\mathbf{h}}_{\mathrm{rp}}-\mathbf{e}^{H} \mathbf{F e} \\
-\sigma_{\mathrm{p}}^{2}+\frac{P_{\mathrm{p}}\left(\Omega_{\mathrm{pp}}-\epsilon_{1}\right)}{\gamma_{\mathrm{T}}} \geq s\left(1-\left\|\frac{\mathbf{e}}{\epsilon_{3}}\right\|_{2}^{2}\right)
\end{array}
$$

which can be rewritten as the quadratic

$$
\exists_{s \geq 0} \mid\left[\begin{array}{ll}
1 & \mathbf{e}^{H}
\end{array}\right] \mathbf{G}\left[\begin{array}{l}
1 \\
\mathbf{e}
\end{array}\right] \geq 0
$$

where $\mathbf{G}$ is defined as

$$
\mathbf{G}=\left[\begin{array}{cc}
-\tilde{\mathbf{h}}_{\mathrm{rp}}^{H} \mathbf{F} \tilde{\mathbf{h}}_{\mathrm{rp}}-\sigma_{\mathrm{p}}^{2}+\frac{P_{\mathrm{p}}\left(\Omega_{\mathrm{pp}}-\epsilon_{1}\right)}{\gamma_{\mathrm{T}}}-s & -\tilde{\mathbf{h}}_{\mathrm{rp}}^{H} \mathbf{F} \\
-\mathbf{F} \tilde{\mathbf{h}}_{\mathrm{rp}} & -\left(\mathbf{F}-\frac{s}{\epsilon_{3}^{2}} \mathbf{I}\right)
\end{array}\right] .
$$


Note that ensuring (6.68) is the same as ensuring that $\mathbf{G} \succeq 0$. Hence, the worst-case $\mathrm{PU}_{\mathrm{Rx}}$ SINR constraint becomes a convex matrix positive semidefinite constraint. Problems (6.8) and (6.12) are transformed into worst-case robust problems by replacing the instantaneous $\mathrm{PU}_{\mathrm{Rx}}$ SINR constraints with $\mathrm{G} \succeq 0$ and the introduction of the auxiliary variable $s . \epsilon_{1}$ and $\epsilon_{3}$ are chosen such that $\operatorname{Pr}\left\{\left|h_{\mathrm{pp}}\right|^{2} \geq \Omega_{\mathrm{pp}}-\epsilon_{1}\right\} \operatorname{Pr}\left\{\|\mathbf{e}\|_{2} \leq \epsilon_{3}\right\}=1-\mathrm{P}_{\mathrm{o}, \max }$. This ensures that the probability of encountering a scenario that the worst-case beamformer has not been designed for is the same as the required outage probability of the proposed methods. In our simulations of the worst-case beamformer, e is a zero-mean vector with independently and identically distributed complex Gaussian entries and the variance of each entry equal to $0.2^{2}\left\|\Omega_{\mathrm{rp}}^{1 / 2}\right\|_{2}^{2} / 8=-8.98 \mathrm{~dB}$. This corresponds to the scenario where $\rho=0.2$ in the simulations of our proposed robust beamformer of Section 6.4.3.

Our proposed robust beamformer of Section 6.4.3 is also compared against a non-robust beamformer. The non-robust beamformer is designed by treating CSI of $\mathbf{h}_{\mathrm{rp}}$ as perfect by ignoring the effects of CSI errors.

In Fig. 6.3, results are provided for the CDF of the $\mathrm{PU}_{\mathrm{Rx}}$ SINR obtained through solving multiple realisations of the SU total relay power minimisation problem (6.8), and the corresponding proposed robust problems (6.25), (6.38) and (6.64). In each realisation of the problem, new instances of the required channels are generated and the beamforming problem is solved to obtain the beamformer weights. This procedure is followed for all results presented in this section. Results are also provided for the worst-case beamformer designs. It can be seen that the required $5 \%$ probability of $\mathrm{PU}_{\mathrm{Rx}}$ SINR being below $5 \mathrm{~dB}$ is satisfied by all three robust optimisation schemes proposed in this paper. Being very conservative, the worst-case designs result in almost zero PU outage probability. A feasible solution for the worst-case beamformer of Section 6.4.2 could not be found, hence results are not shown on the figure. This is because the worst-case method aggressively protects the $\mathrm{PU}_{\mathrm{Rx}}$ and is not able to find a power allocation which guarantees QOS to the $\mathrm{PU}_{\mathrm{Rx}}$ in the worst-case scenario.

Table 6.1 summarises the SU blocking probabilities and the mean total relay power of the various total relay power minimisation problems discussed in this paper. SU blocking probability is defined as the probability that the SU is not able to access the channel, i.e., the probability that the optimisation problem is infeasible due to either SU or PU QoS constraints not being able to be satisfied. We see that increasing channel uncertainty increases the SU blocking probability. The results also show that it is not vital to have the full CSI for the $\mathrm{PU}_{\mathrm{Tx}}$ to $\mathrm{PU}_{\mathrm{Rx}}$ link. Knowledge of the mean channel power of this link only is sufficient to obtain the same SU blocking probability as for the full CSI scenario. It is evident that the worst-case beamformers tend to have much higher SU blocking probabilities 


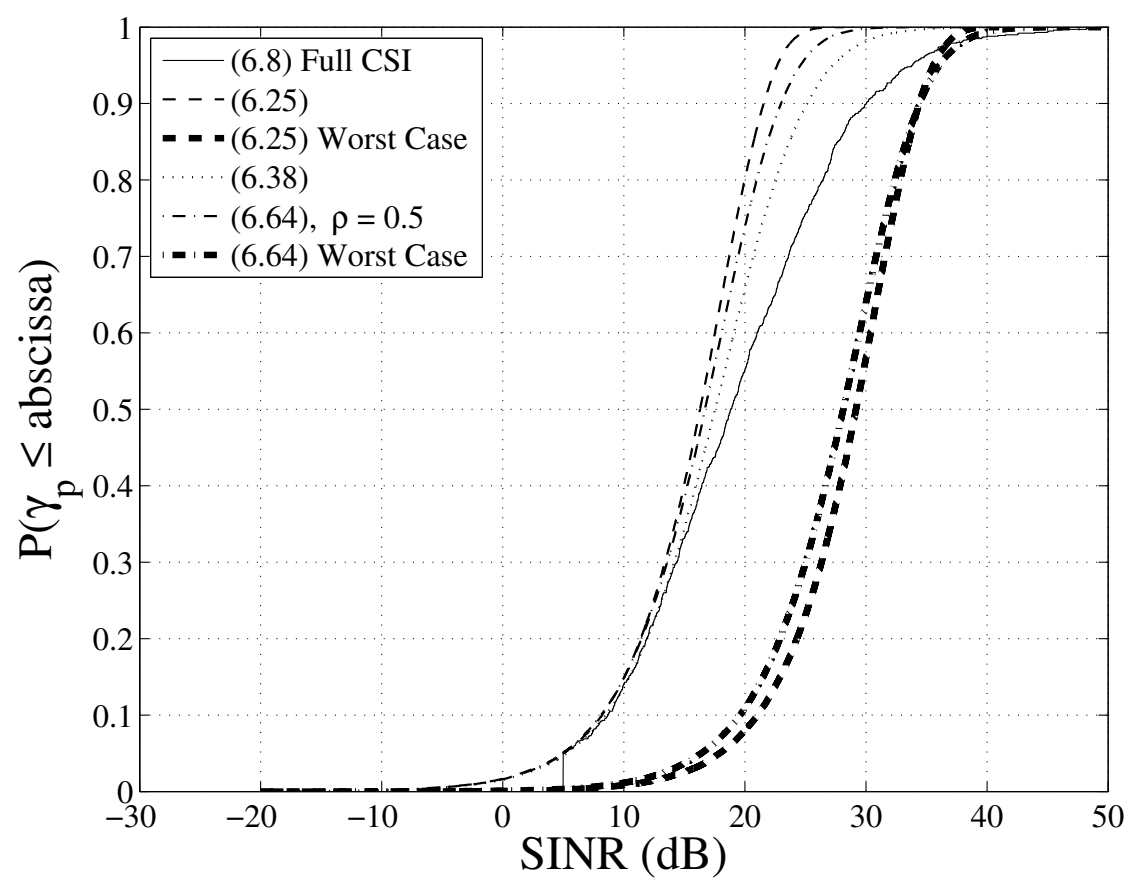

Figure 6.3: SINR at the $\mathrm{PU}_{\mathrm{Rx}}$ for the total relay power minimisation problem.

\begin{tabular}{c|c|c}
\hline \hline Problem & Blocking Probability (\%) & Mean Total Relay Power $(\mathrm{dBm})$ \\
\hline \hline$(6.8)$, Full CSI & 0.2 & -41.0 \\
\hline$(6.25)$ & 0.2 & -41.3 \\
\hline$(6.38)$ & 41 & -46.5 \\
\hline$(6.64), \rho=0.05$ & 0.2 & -41.3 \\
\hline$(6.64), \rho=0.2$ & 0.2 & -41.3 \\
\hline$(6.64), \rho=0.3$ & 2.1 & -41.6 \\
\hline$(6.64), \rho=0.5$ & 15 & -43.6 \\
\hline Sec. 6.4 .1 Worst-Case & 0.3 & -41.6 \\
\hline Sec. 6.4.2 Worst-Case & 100 & - \\
\hline Sec. 6.4.3 Worst-Case & 84 & -54.0 \\
\hline \hline
\end{tabular}

Table 6.1: SU Blocking Probabilities and Mean Relay Power For Total Relay Power Minimisation Problem 
than the robust beamformers proposed in this paper; for instance, the worst-case beamformers of Sections 6.4.2 and 6.4.3 result in blocking probabilities of $100 \%$ and $84 \%$, respectively, which would render them impractical. The results also show that the mean total relay power decreases with increasing channel uncertainty. This is because the channel uncertainty causes the beamformers to become more conservative and the beamformer power is reduced to control interference at the $\mathrm{PU}_{\mathrm{Rx}}$.

In Fig. 6.4, results are provided for the $\mathrm{CDF}$ of the $\mathrm{PU}_{\mathrm{Rx}}$ SINR obtained through solving the $\mathrm{SU}_{\mathrm{Rx}} \mathrm{SINR}$ maximisation problem (6.19), and the corresponding proposed robust problems (6.26), (6.39) and (6.65). Results are also provided for the worst-case designs and a non-robust beamformer design for problem (6.65). The non-robust beamformer treats $\mathbf{h}_{\mathrm{rp}}$ CSI as perfect and ignores the effect of CSI errors in the design process. We see that the outage probability for the full CSI solution is zero. Results show that the $5 \% \mathrm{PU}_{\mathrm{Rx}}$ outage probability requirement is satisfied by all three robust solutions proposed in this paper. The non-robust solution achieves a $\mathrm{PU}_{\mathrm{Rx}}$ outage probability which is greater than $5 \%$ because the outage probability constraint is not respected by this design. Again, the worst-case designs result in very conservative solutions that attain $\mathrm{PU}_{\mathrm{Rx}}$ outage probabilities which are close to zero.

In Fig. 6.5, the output $\mathrm{SU}_{\mathrm{Rx}}$ SINR CDF results for the $\mathrm{SU}_{\mathrm{Rx}}$ SINR maximisation problem (6.19), and the corresponding proposed robust problems (6.26), (6.39) and (6.65) are provided. Results for the worst-case beamformers are also plotted. We see that problems (6.26) and (6.65) ( $\rho=0.05$, see Fig. 6.7 for results for various values of $\rho$ ) result in almost the same performance which is very close to the full CSI scenario. The performance loss due to partial CSI on the $\mathrm{SU}_{\mathrm{Rl}}$ to $\mathrm{PU}_{\mathrm{Rx}}$ link, problem (6.39), is clearly visible. The worst-case beamformer for problem (6.26) results in almost the same performance as the robust design proposed in this paper; however, the worst-case designs for problems (6.39) and (6.65) result in performance that is inferior to our proposed methods.

In Fig. 6.6, the $\mathrm{CDF}$ of the $\mathrm{PU}_{\mathrm{Rx}}$ SINR obtained through solving (6.65) for various values of $\rho$ is provided. The outage probability requirement is satisfied by designs for all three values of $\rho$. We see that the solutions for $\rho=0.3$ and $\rho=0.05$ result in the same $\mathrm{PU}$ performance.

In Fig. 6.7, the CDF of the $\mathrm{SU}_{\mathrm{Rx}} \mathrm{SINR}$ obtained through solving (6.65) for various values of $\rho$ is provided. As a reference, the CDFs of the $\mathrm{SU}_{\mathrm{Rx}} \mathrm{SINR}$ for problems (6.26) and (6.39) are also plotted. As expected, the $\mathrm{SU}_{\mathrm{Rx}}$ performance degrades with increasing CSI error variance. As the CSI error variance increases, the CDF curves are seen to move away from the CDF curve of problem (6.26) and towards that of problem (6.39).

Tables 6.2-6.4 list the ratio of the mean execution time of the linear-fractional 


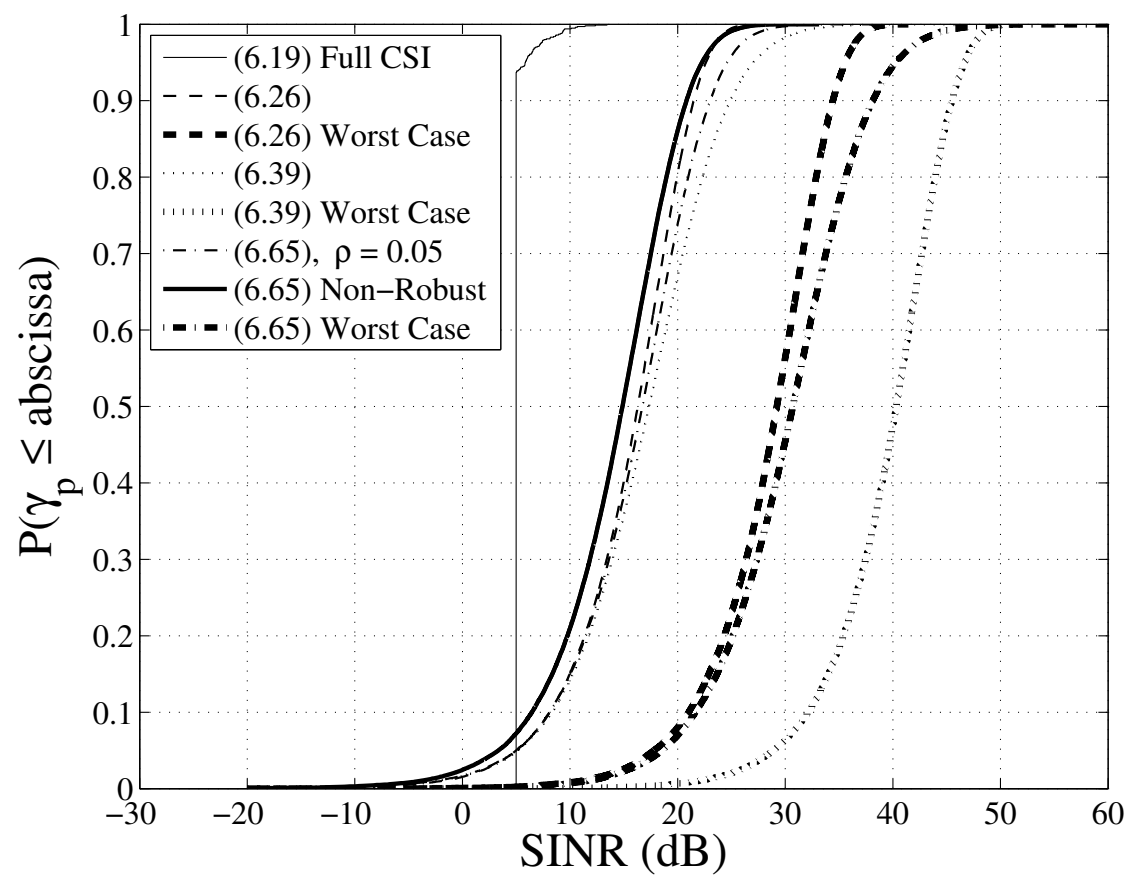

Figure 6.4: $\mathrm{SINR}$ at the $\mathrm{PU}_{\mathrm{Rx}}$ for the $\mathrm{SU}_{\mathrm{Rx}} \mathrm{SINR}$ maximisation problem.

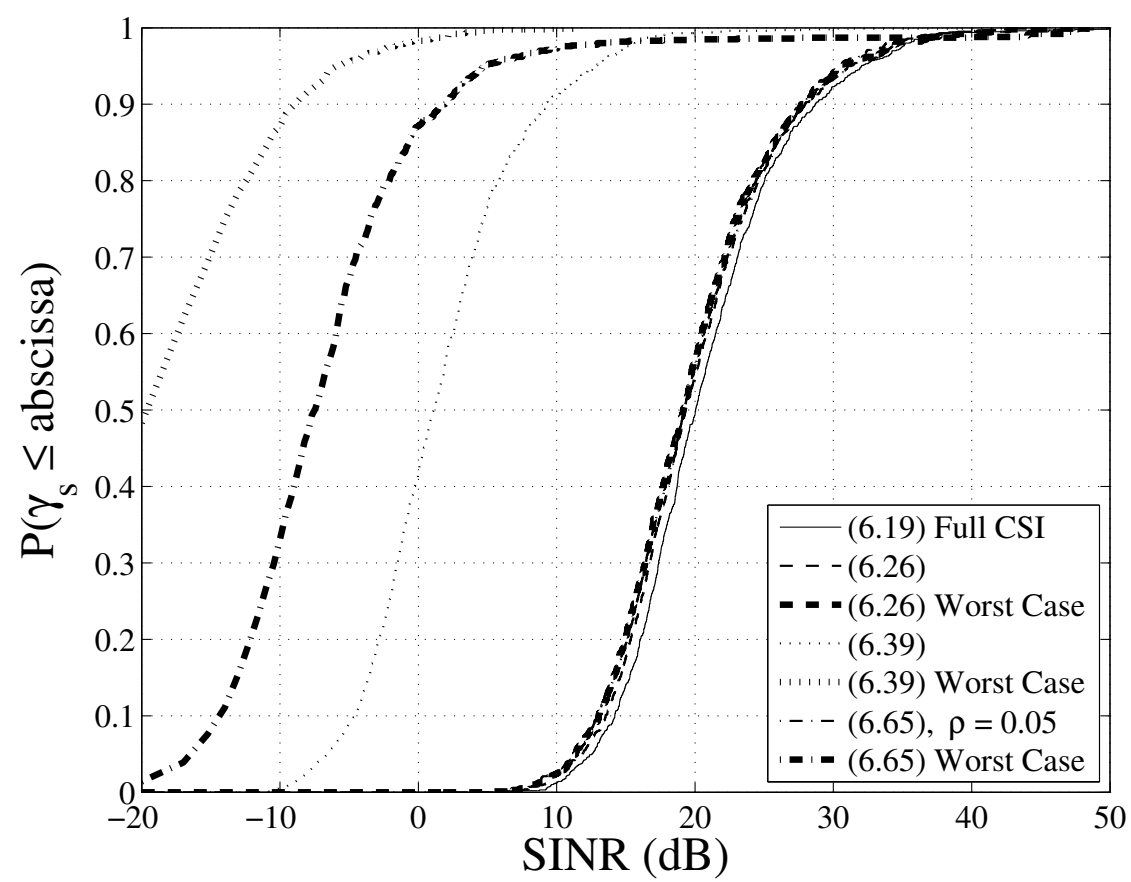

Figure 6.5: $\mathrm{SINR}$ at the $\mathrm{SU}_{\mathrm{Rx}}$ for the $\mathrm{SU}_{\mathrm{Rx}}$ SINR maximisation problem. 


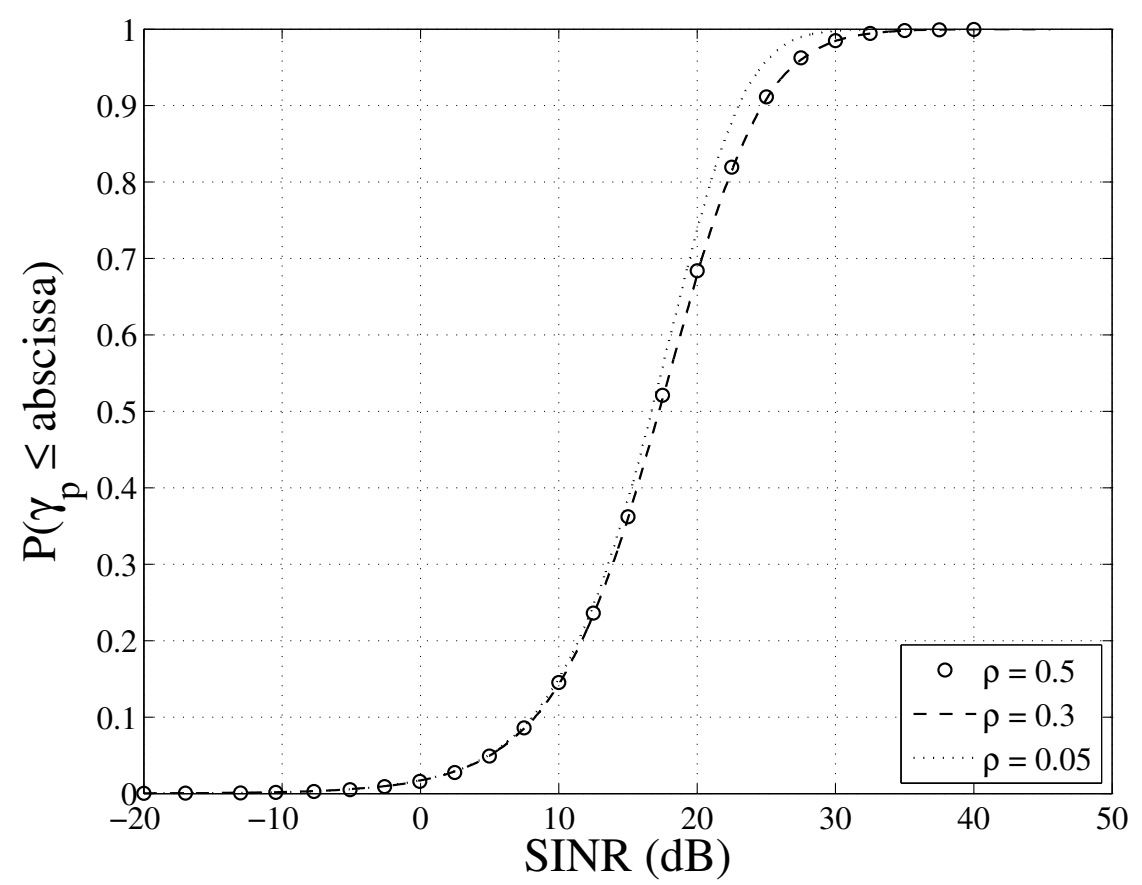

Figure 6.6: SINR at the $\mathrm{PU}_{\mathrm{Rx}}$ for various CSI error level $\rho$ for the $\mathrm{SU}_{\mathrm{Rx}}$ SINR maximisation problem.

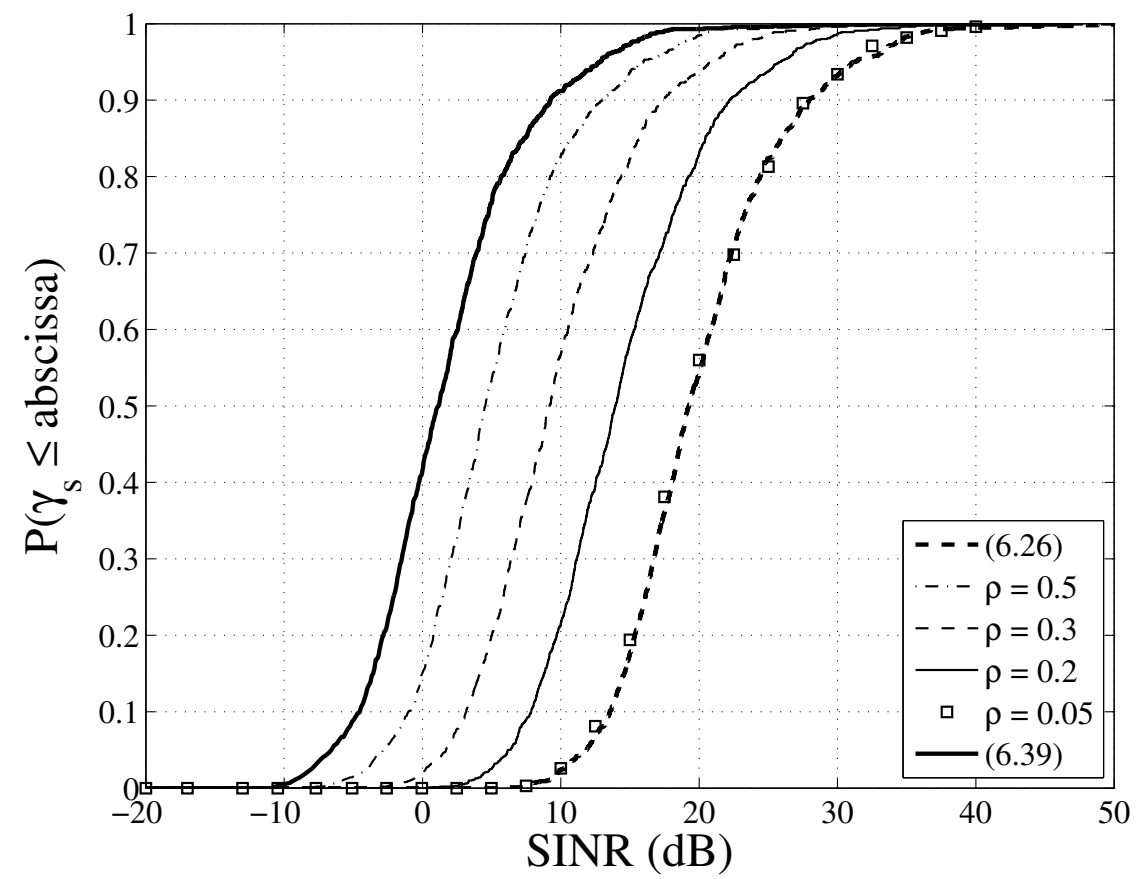

Figure 6.7: $\mathrm{SINR}$ at the $\mathrm{SU}_{\mathrm{Rx}}$ for various CSI error level $\rho$ for the $\mathrm{SU}_{\mathrm{Rx}} \mathrm{SINR}$ maximisation problem. 


\begin{tabular}{c|c|c|c}
\hline \hline SICR (dB) & $R=4$ & $R=8$ & $R=16$ \\
\hline \hline 0.8 & 35.9 & 36.1 & 36.3 \\
\hline \hline 3 & 36.0 & 36.0 & 36.1 \\
\hline \hline 5 & 36.1 & 36.2 & 36.2 \\
\hline \hline 8 & 36.1 & 36.1 & 36.3 \\
\hline \hline
\end{tabular}

Table 6.2: Ratio of mean execution time of linear-fractional program to mean execution time of convex feasibility problem for solving the $\mathrm{SU}_{\mathrm{Rx}}$ SINR maximisation problem under the assumption of full CSI.

\begin{tabular}{c|c|c|c}
\hline \hline SICR (dB) & $R=4$ & $R=8$ & $R=16$ \\
\hline \hline 0.8 & 36.2 & 36.3 & 36.3 \\
\hline \hline 3 & 36.1 & 36.1 & 36.1 \\
\hline \hline 5 & 36.1 & 36.1 & 36.2 \\
\hline \hline 8 & 36.2 & 36.1 & 36.2 \\
\hline \hline
\end{tabular}

Table 6.3: Ratio of mean execution time of linear-fractional program to mean execution time of convex feasibility problem for solving the robust $\mathrm{SU}_{\mathrm{Rx}} \mathrm{SINR}$ maximisation problem (6.65) with $\rho=0.2$.

program method to the mean execution time of the convex feasibility SDP method for solving the $\mathrm{SU}_{\mathrm{Rx}}$ SINR maximisation problem under the assumption of full CSI and its robust counterpart (6.65). The problems are solved using the CVX toolbox [92] for MATLAB. Results are provided for various channel conditions, number of relay nodes and levels of channel uncertainty. The results reveal that in all cases, the linear-fractional program method is on average approximately 36 times faster than the convex feasibility method.

\begin{tabular}{c|c|c|c}
\hline \hline SICR (dB) & $R=4$ & $R=8$ & $R=16$ \\
\hline \hline 0.8 & 36.1 & 36.1 & 36.2 \\
\hline \hline 3 & 36.2 & 36.1 & 36.1 \\
\hline \hline 5 & 36.3 & 36.2 & 36.2 \\
\hline \hline 8 & 36.2 & 36.1 & 36.3 \\
\hline \hline
\end{tabular}

Table 6.4: Ratio of mean execution time of linear-fractional program to mean execution time of convex feasibility problem for solving the robust $\mathrm{SU}_{\mathrm{Rx}} \mathrm{SINR}$ maximisation problem (6.65) with $\rho=0.5$.

\subsection{Summary}

In this chapter, cooperative beamformer problems for a CR relay network have been formulated. The first problem minimises the total relay transmit power subject to $\mathrm{PU}_{\mathrm{Rx}}$ and $\mathrm{SU}_{\mathrm{Rx}}$ QoS constraints. It was shown that this problem can be cast into a convex SOCP and solved efficiently using interior point methods. In the second 
problem, the $\mathrm{SU}_{\mathrm{Rx}} \mathrm{SINR}$ is maximised subject to $\mathrm{PU}_{\mathrm{Rx}}$ QoS and individual relay transmit power constraints. It was demonstrated that this problem can be solved using three methods, namely, a convex feasibility SOCP, a convex feasibility SDP and a SDP that has the form of a linear-fractional program. The linear-fractional program formulation does not require an iterative procedure for solving it, hence, it is the most efficient formulation among the three proposed methods. The execution speed of the linear-fractional program formulation has been found to be on average 36 times faster than the execution speed of the convex feasibility SDP formulation.

New robust counterparts of the cooperative beamformer problems that guarantee a certain $\mathrm{PU}_{\mathrm{Rx}}$ outage probability under the assumption of partial and imperfect CSI have been presented. These problems have the same structure as those formulated under the assumption of perfect CSI, hence, the same methods can be used to solve the robust formulations. Simulation results have shown how the achieved robustness varies with CSI uncertainty. 


\section{Chapter 7}

\section{Robust Cognitive Radio Beamforming}

This chapter considers a $\mathrm{CR}$ network consisting of a $\mathrm{SU}_{\mathrm{Tx}}$ equipped with multiple antennas and a $\mathrm{SU}_{\mathrm{Rx}}$ that share spectrum with multiple $\mathrm{PU}_{\mathrm{Tx}}$ and $\mathrm{PU}_{\mathrm{Rx}}$ pairs. It is assumed that the CR has a low level of cooperation with the primary network and therefore, only partial channel state information of each of the $\mathrm{PU}_{\mathrm{Tx}}$ to $\mathrm{PU}_{\mathrm{Rx}}$ and $\mathrm{SU}_{\mathrm{Tx}}$ to each $\mathrm{PU}_{\mathrm{Rx}}$ links is available. Furthermore, we assume that the $\mathrm{SU}_{\mathrm{Tx}}$ to $\mathrm{SU}_{\mathrm{Rx}}$ link CSI is imperfect, with the channel error modelled as additive Gaussian noise. Under these assumptions, we propose three new robust CR beamformers where i) the total $\mathrm{SU}_{\mathrm{Tx}}$ transmit power is minimised subject to $\mathrm{PU}_{\mathrm{Rx}}$ and $\mathrm{SU}_{\mathrm{Rx}}$ outage probability constraints; ii) the $\mathrm{SU}_{\mathrm{Rx}}$ outage probability is minimised subject to $\mathrm{PU}_{\mathrm{Rx}}$ outage probability and $\mathrm{SU}_{\mathrm{Tx}}$ transmit power constraints; and iii) the maximum $\mathrm{PU}_{\mathrm{Rx}}$ outage probability is minimised subject to $\mathrm{SU}_{\mathrm{Rx}}$ outage probability and $\mathrm{SU}_{\mathrm{Tx}}$ transmit power constraints. We present expressions for $\mathrm{PU}_{\mathrm{Rx}}$ and $\mathrm{SU}_{\mathrm{Rx}}$ outage probabilities and formulate the robust beamformer optimisation problems as convex optimisation problems. $\mathrm{SU}_{\mathrm{Tx}}$ transmit power, $\mathrm{PU}_{\mathrm{Rx}} \mathrm{SINR}$ and $\mathrm{SU}_{\mathrm{Rx}}$ SNR CDFs are obtained through solution of our optimisation problem.

\subsection{Introduction}

As discussed in Section 3.3.3, transmit beamforming has been shown to significantly improve performance in conventional wireless systems $[24,25,41,69,82,84$, 119, 120, 183-185, 199, 204, 205, 209, 211, 212, 235, 247].

Due to its advantages, beamforming has attracted much interest in CR research $[267,268,270,276,277]$. One of the challenges of $\mathrm{CR}$ spectrum sharing is guaranteeing QoS to the PU. Beamforming is seen as a way to overcome this challenge, since the SU can direct its power away from the PU receivers. Design of CR beamformers under the assumption of full CSI has been the subject of investigation in $[267,270]$. In $[268,277]$, the design of robust worst-case CR beamformers 
under the assumption of partial CSI have been studied. Most recently, in [276], the design of a statistically robust CR beamformer under the assumption of imperfect CSI for a CR network with a high level of cooperation with the primary network was addressed and an iterative solution method that involved an exhaustive search was proposed.

In this chapter, we consider the scenario where there are multiple PU transmitter and receiver pairs coexisting with a $\mathrm{SU}_{\mathrm{Tx}}$ and $\mathrm{SU}_{\mathrm{Rx}}$ pair. We assume that the CR network has a low level of cooperation with the primary network and, therefore, only mean channel powers of each of the $\mathrm{PU}_{\mathrm{Tx}}$ to $\mathrm{PU}_{\mathrm{Rx}}$ and the $\mathrm{SU}_{\mathrm{Tx}}$ to each $\mathrm{PU}_{\mathrm{Rx}}$ links are available. Furthermore, we assume that the $\mathrm{SU}_{\mathrm{Tx}}$ to $\mathrm{SU}_{\mathrm{Rx}}$ link CSI is imperfect, with the channel error modelled as additive Gaussian noise. We propose new statistically robust $\mathrm{CR}$ beamformers where either the total $\mathrm{SU}_{\mathrm{Tx}}$ transmit power is minimised subject to $\mathrm{PU}_{\mathrm{Rx}}$ and $\mathrm{SU}_{\mathrm{Rx}}$ outage probability constraints or the maximum $\mathrm{PU}_{\mathrm{Rx}}$ outage probability is minimised subject to $\mathrm{SU}_{\mathrm{Rx}}$ outage probability and total transmit power constraints.

The contributions of this chapter are as follows.

- We first formulate the CR beamforming problem under the assumption of full CSI at the CR system as $\mathrm{SU}_{\mathrm{Tx}}$ transmit power minimisation, $\mathrm{SU}_{\mathrm{Rx}} \mathrm{SNR}$ maximisation and maximisation of the minimum $\mathrm{PU}_{\mathrm{Rx}}$ SINR problems.

- We show that the $\mathrm{SU}_{\mathrm{Tx}}$ transmit power minimisation and the $\mathrm{SU}_{\mathrm{Rx}} \mathrm{SNR}$ maximisation problems can be transformed into convex SOCPs.

- We show that the maximisation of the minimum $\mathrm{PU}_{\mathrm{Rx}} \mathrm{SINR}$ problem can be transformed into a convex feasibility problem with SOC constraints.

- We derive the $\mathrm{PU}_{\mathrm{Rx}}$ and $\mathrm{SU}_{\mathrm{Rx}}$ outage probability expressions.

- We propose new robust beamformers that

1. minimise the $\mathrm{SU}_{\mathrm{Tx}}$ transmit power while guaranteeing certain $\mathrm{PU}_{\mathrm{Rx}}$ and $\mathrm{SU}_{\mathrm{Rx}}$ outage probabilities;

2. minimise the $\mathrm{SU}_{\mathrm{Rx}}$ outage probability while guaranteeing a certain $\mathrm{PU}_{\mathrm{Rx}}$ outage probability and keeping within the $\mathrm{SU}_{\mathrm{Tx}}$ transmit power limits;

3. minimise the maximum $\mathrm{PU}_{\mathrm{Rx}}$ outage probability while guaranteeing a certain $\mathrm{SU}_{\mathrm{Rx}}$ outage probability and keeping within the $\mathrm{SU}_{\mathrm{Tx}}$ transmit power limits;

for the scenarios where i) partial CSI is available for the $\mathrm{PU}_{\mathrm{Tx}}$ to $\mathrm{PU}_{\mathrm{Rx}}$ link and $\mathrm{SU}_{\mathrm{Tx}}$ to each of $\mathrm{PU}_{\mathrm{Rx}}$ links; and ii) imperfect CSI is available for the $\mathrm{SU}_{\mathrm{Tx}}$ to $\mathrm{SU}_{\mathrm{Rx}}$ link. 


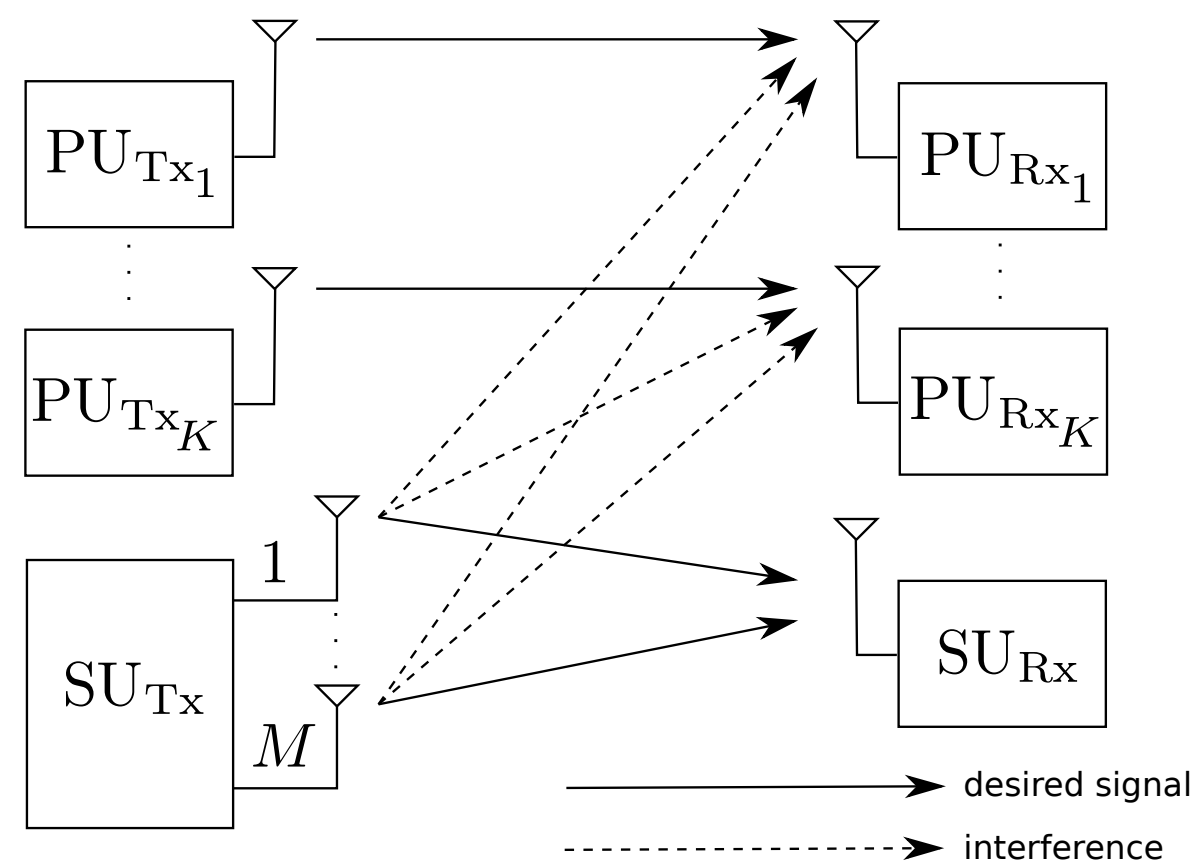

Figure 7.1: System Model

- We show that the robust $\mathrm{SU}_{\mathrm{Tx}}$ transmit power minimisation problem can be transformed into a convex SDP.

- We show that the $\mathrm{SU}_{\mathrm{Rx}}$ outage probability minimisation and the maximum $\mathrm{PU}_{\mathrm{Rx}}$ outage probability minimisation problems can be transformed into convex feasibility problems.

The performance resulting from the optimisation problems outlined above is demonstrated by means of $\mathrm{PU}_{\mathrm{Rx}} \mathrm{SINR}, \mathrm{S} \mathrm{U}_{\mathrm{Rx}} \mathrm{SNR}, \mathrm{SU}_{\mathrm{Tx}}$ transmit power and $\mathrm{SU}_{\mathrm{Rx}}$ and $\mathrm{PU}_{\mathrm{Rx}}$ outage probability CDFs for flat Rayleigh channels.

\subsection{System Model}

As shown in Fig. 7.1, we consider a $\mathrm{CR}$ system which consists of a $\mathrm{SU}_{\mathrm{Tx}}$, a $\mathrm{SU}_{\mathrm{Rx}}$ and $K \mathrm{PU}_{\mathrm{Tx}}$ and $\mathrm{PU}_{\mathrm{Rx}}$ pairs. The $\mathrm{SU}_{\mathrm{Tx}}$ has $M$ antennas while there is only one antenna at the $\mathrm{SU}_{\mathrm{Rx}}$ and each of the $\mathrm{PU}_{\mathrm{Tx}} \mathrm{S}$ and $\mathrm{PU}_{\mathrm{Rx}} \mathrm{s}$. We assume that the $\mathrm{SU}_{\mathrm{Tx}}$ is located in close proximity of the primary system while the $\mathrm{SU}_{\mathrm{Rx}}$ is located at a large distance away from the $\mathrm{PU}_{\mathrm{Tx}} \mathrm{S}$. Hence, in our analysis, we assume that the $\mathrm{PU}_{\mathrm{Tx}}$ transmit powers are sufficiently attenuated by distance to be ignored at the $\mathrm{SU}_{\mathrm{Rx}}$. Since the PU and $\mathrm{SU}$ systems use the same frequency band, the $\mathrm{PU}_{\mathrm{Rx}} \mathrm{S}$ experience interference from the $\mathrm{SU}_{\mathrm{Tx}}$. Due to the low cooperation level between the PU and SU systems, we assume that the SU has no knowledge of the channel access method employed by the PU system. Hence, in our analysis the SU assumes that all $\mathrm{PU}_{\mathrm{Tx}} \mathrm{S}$ are active simultaneously.

Independent, point-to-point, flat Rayleigh fading channels are assumed for all 
links in the network. The channel between the $i$ th $\mathrm{PU}_{\mathrm{Tx}}$ and the $i$ th $\mathrm{PU}_{\mathrm{Rx}}$ and the $\mathrm{SU}_{\mathrm{Tx}}$ and $i$ th $\mathrm{PU}_{\mathrm{Rx}}$ are denoted by the scalar $c_{i} \in \mathbb{C}$ and the vector $\mathbf{f}_{i} \in \mathbb{C}^{M \times 1}$, respectively, for $i=1 \ldots K . \mathbf{h} \in \mathbb{C}^{M \times 1}$ denotes the channel between the $\mathrm{SU}_{\mathrm{Tx}}$ and the $\mathrm{SU}_{\mathrm{Rx}}$. The instantaneous channel powers of these links are represented by $g_{c}^{(i)}=\left|c_{i}\right|^{2}, g_{\mathrm{f}_{i}}^{(j)}=\left|f_{i}^{(j)}\right|^{2}$ and $g_{\mathrm{h}}^{(j)}=\left|h^{(j)}\right|^{2}$ for $j=1 \ldots M$ and are governed by their corresponding parameters $\mathbb{E}\left\{g_{c}^{(i)}\right\}=\Omega_{c}^{(i)}, \mathbb{E}\left\{g_{\mathrm{f}_{i}}^{(j)}\right\}=\Omega_{\mathrm{f}_{i}}^{(j)}$ and $\mathbb{E}\left\{g_{\mathrm{h}}^{(j)}\right\}=\Omega_{\mathrm{h}}^{(j)}$.

The signal at the $\mathrm{SU}_{\mathrm{Rx}}$ is given by

$$
y=\mathbf{h}^{H} \mathbf{w} \sqrt{P_{\mathrm{s}}} s_{\mathrm{s}}+n_{\mathrm{s}},
$$

and that at the $i$ th $\mathrm{PU}_{\mathrm{Rx}}$ by

$$
z_{i}=\sqrt{P_{\mathrm{p}}} s_{\mathrm{p}}^{(i)} c_{i}+\mathbf{f}_{i}^{H} \mathbf{w} \sqrt{P_{\mathrm{s}}} s_{\mathrm{s}}+n_{\mathrm{p}}^{(i)}
$$

where $\sqrt{P_{\mathrm{s}}} s_{\mathrm{s}}$ is information symbol transmitted by the $\mathrm{SU}_{\mathrm{Tx}}, \mathbf{w} \in \mathbb{C}^{M \times 1}$ is the beamforming vector at the $\mathrm{SU}_{\mathrm{Tx}}$ and $n_{\mathrm{s}}$ is the AWGN with variance $\sigma_{\mathrm{s}}^{2}$ at the $\mathrm{SU}_{\mathrm{Rx}}, P_{\mathrm{p}}$ is the $\mathrm{PU}_{\mathrm{Tx}}$ transmit power, $s_{\mathrm{p}}^{(i)}$ is the information symbol transmitted by the $i$ th $\mathrm{PU}_{\mathrm{Tx}}$ and $n_{\mathrm{p}}^{(i)}$ is the AWGN with variance $\sigma_{\mathrm{p}}^{2}$, at the $i$ th $\mathrm{PU}_{\mathrm{Rx}}$. We assume $\mathbb{E}\left\{\left|\sqrt{P_{\mathrm{s}}} s_{\mathrm{s}}\right|^{2}\right\}=\mathbb{E}\left\{\left|s_{\mathrm{p}}^{(i)}\right|^{2}\right\}=1$.

The power transmitted by the $\mathrm{SU}_{\mathrm{Tx}}$ is given by

$$
P_{T}=\|\mathbf{w}\|_{2}^{2}
$$

Using (7.1) and (7.2) and assuming that $\sqrt{P_{\mathrm{s}}} s_{\mathrm{s}}$ and $\sqrt{P_{\mathrm{p}}} s_{\mathrm{p}}^{(i)}$, are uncorrelated, the $\mathrm{SNR}$ at the $\mathrm{SU}_{\mathrm{Rx}}$ can be expressed as

$$
\gamma_{\mathrm{s}}=\frac{\left|\mathbf{h}^{H} \mathbf{w}\right|^{2}}{\sigma_{\mathrm{s}}^{2}}
$$

and the SINR at the $i$ th $\mathrm{PU}_{\mathrm{Rx}}$ is given by

$$
\gamma_{\mathrm{p}}^{(i)}=\frac{P_{\mathrm{p}}\left|c_{i}\right|^{2}}{\left|\mathbf{f}_{i}^{H} \mathbf{w}\right|^{2}+\sigma_{\mathrm{p}}^{2}} .
$$

In an underlay cognitive radio system the secondary users are allowed to operate as long as they can guarantee a certain level of quality of service to the primary user. Hence, in our analysis we impose an SINR constraint, $\gamma_{\mathrm{T}}^{(i)}$, at the $i$ th $\mathrm{PU}_{\mathrm{Rx}}$, i.e., $\gamma_{\mathrm{p}}^{(i)} \geq \gamma_{\mathrm{T}}^{(i)}$.

\subsection{Beamformer Optimisation Under Full CSI}

In this section, we aim to find the optimum beamforming weight vector, w, such that i) the total $\mathrm{SU}_{\mathrm{Tx}}$ transmit power, $P_{T}$, is minimised while guaranteeing mini- 
mum QoS to the $\mathrm{SU}_{\mathrm{Rx}}$ and each of the $\mathrm{PU}_{\mathrm{Rx}} \mathrm{S}$; or ii) the minimum $\mathrm{PU}_{\mathrm{Rx}}$ SINR is maximised subject to $\mathrm{SU}_{\mathrm{Rx}}$ minimum QoS and peak transmit power constraints.

In our analysis, we assume that we are unable to control the PU's transmit power and that all of the PUs transmit at a constant power of $P_{\mathrm{p}}$. In this section, we formulate the beamforming problems under the assumption that full CSI for all links is available. This is the basis for the development of the robust beamformers in Section 7.4.

\subsubsection{SU Transmitter Power Minimisation}

The total $\mathrm{SU}_{\mathrm{Tx}}$ transmit power minimisation problem can be mathematically represented as

$$
\begin{array}{ll}
\min _{\mathbf{w}} & \|\mathbf{w}\|_{2} \\
\text { s.t. } & \frac{P_{\mathrm{p}}\left|c_{i}\right|^{2}}{\left|\mathbf{f}_{i}^{H} \mathbf{w}\right|^{2}+\sigma_{\mathrm{p}}^{2}} \geq \gamma_{\mathrm{T}}^{(i)}, \quad i=1 \ldots K \\
& \frac{\left|\mathbf{h}^{H} \mathbf{w}\right|^{2}}{\sigma_{\mathrm{s}}^{2}} \geq \gamma_{\mathrm{s}, \min } .
\end{array}
$$

Constraint (7.6c) is satisfied with equality at the optimum. Otherwise, the optimum w could be scaled down to satisfy the constraint with equality, hence decreasing the objective function and contradicting optimality.

Problem (7.6) is a non-convex optimisation problem, but it can be reformulated into a convex optimisation problem. Following [25], we observe that neither the objective function nor the constraints change if the beamforming vector undergoes a phase rotation. Thus, $\mathbf{h}^{H} \mathbf{w}$ can be chosen to be real without loss of generality. The transmit power minimisation problem can therefore be restated as the following SOCP

$$
\begin{array}{ll}
\min _{\mathbf{w}} & \|\mathbf{w}\|_{2} \\
\text { s.t. } & \sqrt{P_{\mathrm{p}}\left|c_{i}\right|^{2}} \geq \sqrt{\gamma_{\mathrm{T}}^{(i)}}\left\|\mathbf{f}_{i}^{H} \mathbf{w}\right\|_{\mathrm{p}} \|_{2}, \quad i=1 \ldots K \\
& \mathbf{h}^{H} \mathbf{w} \geq \sigma_{\mathrm{s}} \sqrt{\gamma_{\mathrm{s}, \min }} .
\end{array}
$$

In the interest of brevity, the further constraints $\Re\left\{\mathbf{h}^{H} \mathbf{w}\right\} \geq 0$ and $\Im\left\{\mathbf{h}^{H} \mathbf{w}\right\}=0$, are not explicitly stated in any of the SOCPs in the following sections. Problem (7.7) is in a convex form and standard interior point methods can be used to solve it efficiently. 


\subsubsection{SU Receiver SNR Maximisation}

In the $\mathrm{SU}_{\mathrm{Rx}} \mathrm{SNR}$ maximisation problem, the received signal power at the $\mathrm{SU}_{\mathrm{Rx}}$ is maximised while placing an upper limit on the total $\mathrm{SU}_{\mathrm{Tx}}$ transmit power and guaranteeing QoS to the $\mathrm{PU}_{\mathrm{Rx}} \mathrm{s}$. This is mathematically stated as

$$
\begin{array}{cl}
\max _{\mathbf{w}} & \left|\mathbf{h}^{H} \mathbf{w}\right|^{2} \\
\text { s.t. } & (7.6 \mathrm{~b}), \\
& \|\mathbf{w}\|_{2}^{2} \leq P_{T, \max } .
\end{array}
$$

Problem (7.8) is a non-convex optimisation problem; however, again noting that neither the objective function nor the constraints change if the beamforming vector undergoes a phase rotation allows us to choose $\mathbf{h}^{H} \mathbf{w}$ to be real. Hence, problem (7.8) can be transformed into the following convex SOCP

$$
\begin{array}{ll}
\max _{\mathbf{w}} & \mathbf{h}^{H} \mathbf{w} \\
\text { s.t. } & (7.7 \mathrm{~b}), \\
& \sqrt{P_{T, \max }} \geq\|\mathbf{w}\|_{2} .
\end{array}
$$

\subsubsection{Minimum PU Receiver SINR Maximisation}

In some cases the SU system would design the beamforming vector such that the minimum $\mathrm{PU}_{\mathrm{Rx}} \mathrm{SINR}$ is maximised while meeting its own performance requirements. This problem is stated as

$$
\begin{array}{ll}
\max _{\mathbf{w}} & \min _{i=1, \ldots, K}\left(\frac{P_{\mathrm{p}}\left|c_{i}\right|^{2}}{\left|\mathbf{f}_{i}^{H} \mathbf{w}\right|^{2}+\sigma_{\mathrm{p}}^{2}}\right) \\
\text { s.t. } & (7.6 \mathrm{c}), \\
& \|\mathbf{w}\|_{2}^{2} \leq P_{T, \max } .
\end{array}
$$

Using the epigraph form, problem (7.10) can be restated as

$$
\begin{aligned}
& \max _{t, \mathbf{w}} \quad t \\
& \text { s.t. } \quad \frac{P_{\mathrm{p}}\left|c_{i}\right|^{2}}{\left|\mathbf{f}_{i}^{H} \mathbf{w}\right|^{2}+\sigma_{\mathrm{p}}^{2}} \geq t, \quad i=1 \ldots K \\
& \text { (7.6c), } \\
& \|\mathbf{w}\|_{2}^{2} \leq P_{T, \max } .
\end{aligned}
$$

We again see that neither the objective function nor the constraints change if the beamforming vector undergoes a phase rotation. Thus, $\mathbf{h}^{H} \mathbf{w}$ can be chosen to be 
real. Problem (7.11) can therefore be written as

$$
\begin{array}{rl}
\max _{t, \mathbf{w}} & t \\
\text { s.t. } & \sqrt{P_{\mathrm{p}}\left|c_{i}\right|^{2}} \geq \sqrt{t}\left\|\mathbf{f}_{i}^{H} \mathbf{w} \sigma_{\mathrm{p}}\right\|_{2}, \quad i=1 \ldots K \\
& (7.7 \mathrm{c}), \\
& \sqrt{P_{T, \max }} \geq\|\mathbf{w}\|_{2} .
\end{array}
$$

Due to coupling between the optimisation variables, constraint $(7.12 \mathrm{~b})$ is a nonconvex constraint. However, as was seen in Sections 3.3.4 and 6.3.2, for any fixed value of $t$ the set of feasible $\mathbf{w}$ is convex and hence the problem is quasi convex. Therefore, for some given $t$, problem (7.12) can be expressed as the following convex feasibility problem with SOCP constraints

$$
\begin{array}{ll}
\text { find } & \mathbf{w} \\
\text { s.t. } & (7.12 \mathrm{~b}),(7.12 \mathrm{c}) \text { and }(7.12 \mathrm{~d}) .
\end{array}
$$

The bisection method [29] can be used to solve problem (7.13) in an iterative manner.

\subsection{Robust Beamformer Optimisation Under Par- tial And Imperfect CSI}

In practice, full CSI for all links is seldom available and the assumption of full or perfect CSI may be overly idealistic. We consider a CR network with a low cooperation level with the primary network. We assume that only mean channel powers of each of the $\mathrm{PU}_{\mathrm{Tx}}$ to $\mathrm{PU}_{\mathrm{Rx}}$ and the $\mathrm{SU}_{\mathrm{Tx}}$ to each $\mathrm{PU}_{\mathrm{Rx}}$ links are available, i.e., only $\Omega_{c}^{(i)} \forall i$ and $\Omega_{\mathrm{f}_{i}}^{(j)} \forall i, j$ for the aforementioned links are available. Furthermore, we assume that the CSI of the $\mathrm{SU}_{\mathrm{Tx}}$ to $\mathrm{SU}_{\mathrm{Rx}}$ link is imperfect. The imperfection may be due to estimation errors or other factors such as quantisation. In our analysis, we model the CSI errors as additive complex Gaussian noise. Hence, using the imperfect CSI model (6.40), we have

$$
\mathbf{h}=\tilde{\mathbf{h}}+\rho \mathbf{e}
$$

where $\tilde{\mathbf{h}}$ is the imperfect $\mathrm{SU}_{\mathrm{Tx}}$ to $\mathrm{SU}_{\mathrm{Rx}}$ link CSI estimate known at the $\mathrm{SU}_{\mathrm{Tx}}$ and e is the zero mean estimation error vector with independently distributed complex Gaussian entries and the diagonal covariance matrix $\boldsymbol{\Sigma}_{\mathrm{e}}=\left(\left\|\boldsymbol{\Omega}_{\mathrm{h}}^{1 / 2}\right\|_{2}^{2} / M\right) \mathbf{I}$, i.e., $\mathbf{e} \sim$ $\mathcal{N}_{\mathcal{C}}\left(\mathbf{0}, \boldsymbol{\Sigma}_{\mathrm{e}}\right)$. Here, $\boldsymbol{\Omega}_{\mathrm{h}}=\left[\Omega_{\mathrm{h}}^{(1)} \Omega_{\mathrm{h}}^{(2)} \ldots \Omega_{\mathrm{h}}^{(M)}\right]^{T} .0 \leq \rho \leq\left(\min \left(\boldsymbol{\Omega}_{\mathrm{h}}\right) /\left(\left\|\boldsymbol{\Omega}_{\mathrm{h}}^{1 / 2}\right\|_{2}^{2} / M\right)\right)^{1 / 2}$ determines the quality of the CSI, which is perfect when $\rho=0$ and has maximum 
uncertainty when $\rho=\left(\min \left(\boldsymbol{\Omega}_{\mathrm{h}}\right) /\left(\left\|\boldsymbol{\Omega}_{\mathrm{h}}^{1 / 2}\right\|_{2}^{2} / M\right)\right)^{1 / 2}$.

In our formulation we consider the SU and PU outage probability as the QoS parameter. In the system under consideration, outage at the $i$ th PU occurs when its SINR, $\gamma_{\mathrm{p}}^{(i)}$, falls below the threshold $\gamma_{\mathrm{T}}^{(i)}$. Similarly, SU outage occurs when the SU SNR, $\gamma_{\mathrm{s}}$, falls below the SU SNR threshold, $\gamma_{\mathrm{s}, \mathrm{min}}$. The $i$ th PU outage probability is expressed as

$$
\begin{aligned}
\mathrm{P}_{\mathrm{o}}^{(i)} & =\operatorname{Pr}\left\{\gamma_{\mathrm{p}}^{(i)} \leq \gamma_{\mathrm{T}}^{(i)}\right\} \\
& =\operatorname{Pr}\left\{P_{\mathrm{p}}\left|c_{i}\right|^{2}-\gamma_{\mathrm{T}}^{(i)} \mathbf{w}^{H} \mathbf{f}_{i} \mathbf{f}_{i}^{H} \mathbf{w} \leq \gamma_{\mathrm{T}}^{(i)} \sigma_{\mathrm{p}}^{2}\right\}
\end{aligned}
$$

where we have used the relation $\left|\mathbf{f}_{i}^{H} \mathbf{w}\right|^{2}=\mathbf{w}^{H} \mathbf{f}_{i} \mathbf{f}_{i}^{H} \mathbf{w}$. Likewise, the SU outage probability is expressed as

$$
\mathrm{P}_{\mathrm{o}}^{\mathrm{SU}}=\operatorname{Pr}\left\{\left|\mathbf{h}^{H} \mathbf{w}\right|^{2} \leq \gamma_{\mathrm{s}, \min } \sigma_{\mathrm{s}}^{2}\right\}
$$

The probabilistic measures are performed over $c_{i}$ and $\mathbf{f}_{i}$ statistics in (7.15) and over CSI error statistics in (7.16).

To proceed, we observe that, in (7.15), we are dealing with a PDF that is given by the difference of two random variables, namely, $P_{\mathrm{p}}\left|c_{i}\right|^{2}$ and $\gamma_{\mathrm{T}}^{(i)} \mathbf{w}^{H} \mathbf{f}_{i} \mathbf{f}_{i}^{H} \mathbf{w}$. It is easily shown that $P_{\mathrm{p}}\left|c_{i}\right|^{2}$ has an exponential distribution with a mean of $P_{\mathrm{p}} \Omega_{c}^{(i)}$.

We note that $\gamma_{\mathrm{T}}^{(i)} \mathbf{W}^{H} \mathbf{f}_{i} \mathbf{f}_{i}^{H} \mathbf{w}=\gamma_{\mathrm{T}}^{(i)} \mathbf{f}_{i}^{H} \mathbf{W} \mathbf{f}_{i}$, where $\mathbf{W}=\mathbf{w} \mathbf{w}^{H}$. Using Lemma 6.4.1 in Chapter 6, page 107, and exploiting the fact that $\mathbf{W}$ is a rank-one matrix, we have that $\gamma_{\mathrm{T}}^{(i)} \mathbf{w}^{H} \mathbf{f}_{i} \mathbf{f}_{i}^{H} \mathbf{w}$ is exponentially distributed with a mean of $\gamma_{\mathrm{T}}^{(i)} \operatorname{tr}\left(\boldsymbol{\Sigma}_{\mathbf{f}_{i}} \mathbf{W}\right)$, where $\boldsymbol{\Sigma}_{\mathbf{f}_{i}}$ is the covariance matrix of $\mathbf{f}_{i}$ and is expressed as

$$
\boldsymbol{\Sigma}_{\mathbf{f}_{i}}=\operatorname{diag}\left(\boldsymbol{\Omega}_{\mathrm{f}_{i}}\right)
$$

where $\Omega_{\mathrm{f}_{i}}=\left[\Omega_{\mathrm{f}_{i}}^{(1)} \Omega_{\mathrm{f}_{i}}^{(2)} \ldots \Omega_{\mathrm{f}_{i}}^{(M)}\right]^{T}$.

The PDF in (7.15) is that of a difference between two independent exponential random variables and can easily be shown to have the following form

$$
f(\psi)= \begin{cases}\frac{\lambda_{1} \lambda_{2}}{\lambda_{1}+\lambda_{2}} \exp \left(-\lambda_{1} \psi\right) & \text { if } \psi \geq 0 \\ \frac{\lambda_{1} \lambda_{2}}{\lambda_{1}+\lambda_{2}} \exp \left(\lambda_{2} \psi\right) & \text { if } \psi<0\end{cases}
$$

where $\lambda_{1}=1 /\left(P_{\mathrm{p}} \Omega_{c}^{(i)}\right)$ and $\lambda_{2}=1 /\left(\gamma_{\mathrm{T}}^{(i)} \operatorname{tr}\left(\boldsymbol{\Sigma}_{\mathbf{f}_{i}} \mathbf{W}\right)\right)$.

Using (7.18) and utilising the fact that $\gamma_{\mathrm{T}}^{(i)} \sigma_{\mathrm{p}}^{2} \geq 0,(7.15)$ can be rewritten as

$$
\begin{aligned}
\mathrm{P}_{\mathrm{o}}^{(i)} & =1-\int_{\gamma_{\mathrm{T}}^{(i)} \sigma_{\mathrm{p}}^{2}}^{\infty} \frac{\lambda_{1} \lambda_{2}}{\lambda_{1}+\lambda_{2}} \exp \left(-\lambda_{1} \psi\right) \mathrm{d} \psi \\
& =1-\frac{\lambda_{2}}{\lambda_{1}+\lambda_{2}} \exp \left(-\lambda_{1} \gamma_{\mathrm{T}}^{(i)} \sigma_{\mathrm{p}}^{2}\right)
\end{aligned}
$$




$$
=1-\frac{P_{\mathrm{p}} \Omega_{c}^{(i)}}{\gamma_{\mathrm{T}}^{(i)} \operatorname{tr}\left(\boldsymbol{\Sigma}_{\mathbf{f}_{i}} \mathbf{W}\right)+P_{\mathrm{p}} \Omega_{c}^{(i)}} \exp \left(-\frac{\gamma_{\mathrm{T}}^{(i)} \sigma_{\mathrm{p}}^{2}}{P_{\mathrm{p}} \Omega_{c}^{(i)}}\right) .
$$

In (7.16), $\left|\mathbf{h}^{H} \mathbf{w}\right|^{2}$ is recognised to be a non-central Chi-square random variable whose CDF is given by the generalised Marcum's Q function [179]. The generalised Marcum's Q function is difficult to handle and so to obtain a mathematically tractable solution, we rewrite (7.16) as follows

$$
\begin{gathered}
\mathrm{P}_{\mathrm{o}}{ }^{\mathrm{SU}}=\operatorname{Pr}\left\{\mathbf{w}^{H} \tilde{\mathbf{h}} \tilde{\mathbf{h}}^{H} \mathbf{w}+\rho \mathbf{w}^{H}\left(\tilde{\mathbf{h}} \mathbf{e}^{H}+\mathbf{e}^{H}\right) \mathbf{w}\right. \\
\left.+\rho^{2} \mathbf{w}^{H} \mathbf{e} \mathbf{e}^{H} \mathbf{w} \leq \gamma_{\mathrm{s}, \min } \sigma_{\mathrm{s}}^{2}\right\} \\
=\operatorname{Pr}\{\underbrace{2 \rho \Re\left\{\mathbf{w}^{H} \tilde{\mathbf{h}} \mathbf{e}^{H} \mathbf{w}\right\}}_{u}+\underbrace{\rho^{2} \mathbf{w}^{H} \mathbf{e e}^{H} \mathbf{w}}_{v} \\
\left.\leq \gamma_{\mathrm{s}, \min } \sigma_{\mathrm{s}}^{2}-\mathbf{w}^{H} \tilde{\mathbf{h}} \tilde{\mathbf{h}}^{H} \mathbf{w}\right\} .
\end{gathered}
$$

Since $u$ in (7.20) is a linear combination of zero-mean independent Gaussian random variables, it itself is a zero-mean Gaussian random variable with variance $\sigma_{u}^{2}$ given by

$$
\begin{aligned}
\sigma_{u}^{2} & =4 \mathbb{E}\left\{\rho^{2} \mathbf{w}^{H} \tilde{\mathbf{h}} \mathbf{e}^{H} \mathbf{w} \mathbf{w}^{H} \mathbf{e} \tilde{\mathbf{h}}^{H} \mathbf{w}\right\} \\
& =4 \rho^{2} \mathbf{w}^{H} \tilde{\mathbf{h}} \mathbf{w}^{H} \mathbb{E}\left\{\mathbf{e} e^{H}\right\} \mathbf{w} \tilde{\mathbf{h}}^{H} \mathbf{w} \\
& =4 \rho^{2} \operatorname{tr}\left(\tilde{\mathbf{h}} \tilde{\mathbf{h}}^{H} \mathbf{W}\right) \operatorname{tr}\left(\boldsymbol{\Sigma}_{\mathrm{e}} \mathbf{W}\right) \\
& =4 \rho^{2} \operatorname{tr}\left(\tilde{\mathbf{h}} \tilde{\mathbf{h}}^{H} \mathbf{W} \boldsymbol{\Sigma}_{\mathrm{e}} \mathbf{W}\right) \\
& =4 \rho^{2}\left\|\left(\boldsymbol{\Sigma}_{\mathrm{e}} \tilde{\mathbf{h}} \tilde{\mathbf{h}}^{H}\right)^{\frac{1}{2}} \mathbf{W}\right\|_{2}^{2},
\end{aligned}
$$

where $\mathbf{W}=\mathbf{w w}^{H}$. Using Lemma 6.4.1 in Chapter 6, page 107, $v$ in (7.20) is recognised as an exponentially distributed random variable with mean $\mu_{v}=\rho^{2} \operatorname{tr}\left(\boldsymbol{\Sigma}_{\mathrm{e}} \mathbf{W}\right)$ and variance $\sigma_{v}^{2}=\rho^{4} \operatorname{tr}\left(\boldsymbol{\Sigma}_{\mathrm{e}} \mathbf{W}\right)^{2}$.

When channel uncertainty is large, beamforming loses its effectiveness since the beam width has to be widened to handle the channel uncertainty. In this work we focus on the scenario where the channel uncertainty is in the acceptable range for beamforming to be practical. In this scenario, $\sigma_{v}^{2}$ is much smaller than $\sigma_{u}^{2}$ and as a result the PDF in (7.20) is dominated by the Gaussian random variable $v$. Therefore, the PDF in (7.20) can be approximated by the zero-mean Gaussian distribution with variance $\sigma_{u}^{2}$. Figs. 7.2-7.6 compares the true distribution obtained using the Marcum's Q function with its Gaussian approximation for various values of $\rho$. In all cases there are 4 transmit antennas at the $\mathrm{SU}_{\mathrm{Tx}}$ and all entries of $\boldsymbol{\Omega}_{\mathrm{h}}$ are equal. As a reference, maximum uncertainty occurs when $\rho=1$. From the figures, it is evident that the approximation provides a good representation of the true CDF for values of $\rho$ less than 0.4 . 


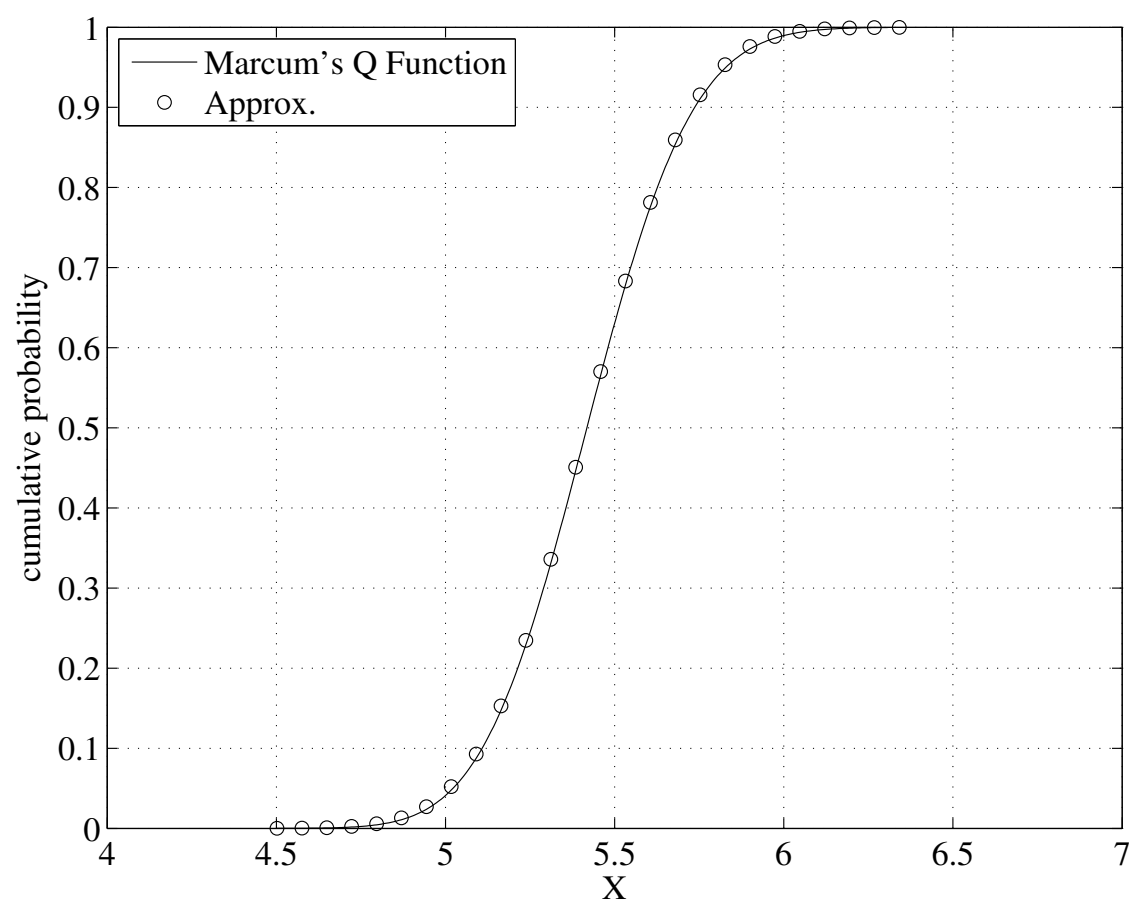

Figure 7.2: True CDF and approximation for $\rho=0.05$.

The SU outage probability can therefore be approximated as

$$
\mathrm{P}_{\mathrm{o}}^{\mathrm{SU}} \approx 1-\frac{1}{2} \operatorname{erfc}\left(\frac{\gamma_{\mathrm{s}, \min } \sigma_{\mathrm{s}}^{2}-\operatorname{tr}\left(\tilde{\mathbf{h}} \tilde{\mathbf{h}}^{H} \mathbf{W}\right)}{2 \rho\left\|\left(\boldsymbol{\Sigma}_{\mathrm{e}} \tilde{\mathbf{h}}^{H}\right)^{\frac{1}{2}} \mathbf{W}\right\|_{2}}\right) .
$$

\subsubsection{Robust SU Transmitter Power Minimisation}

To develop the robust transmit power minimisation problem, the PU SINR and the SU SNR constraints in problem (7.6) are replaced by outage probability constraints, i.e., given maximum allowable PU and SU outage probabilities, $\alpha^{(i)}$ and $\beta$, constraints (7.6b) and (7.6c) are replaced with $\mathrm{P}_{\mathrm{o}}{ }^{(i)} \leq \alpha^{(i)}$ and $\mathrm{P}_{\mathrm{o}}{ }^{\mathrm{SU}} \leq \beta$, respectively. Using (7.19), the $i$ th PU outage probability constraint can be written as the following convex constraint

$$
\operatorname{tr}\left(\boldsymbol{\Sigma}_{\mathbf{f}_{i}} \mathbf{W}\right) \leq \frac{P_{\mathrm{p}} \Omega_{c}^{(i)}\left(\exp \left(-\frac{\gamma_{\mathrm{T}}^{(i)} \sigma_{\mathrm{p}}^{2}}{P_{\mathrm{p}} \Omega_{c}^{(i)}}\right)-1+\alpha^{(i)}\right)}{\gamma_{\mathrm{T}}^{(i)}\left(1-\alpha^{(i)}\right)} .
$$

An important observation in the above constraint is that it is dependent only on the diagonal elements of $\mathbf{W}$, i.e., dependent only on the beamformer transmit power. This is a fairly intuitive result since phase information of the $\mathrm{SU}_{\mathrm{Tx}}$ to $\mathrm{PU}_{\mathrm{Rx}}$ links are not available and, therefore, power control is the only degree of freedom available to the beamformer to control the amount of interference to the $\mathrm{PU}_{\mathrm{Rx}} \mathrm{S}$.

Similarly, using (7.22), the SU outage probability constraint can be expressed 


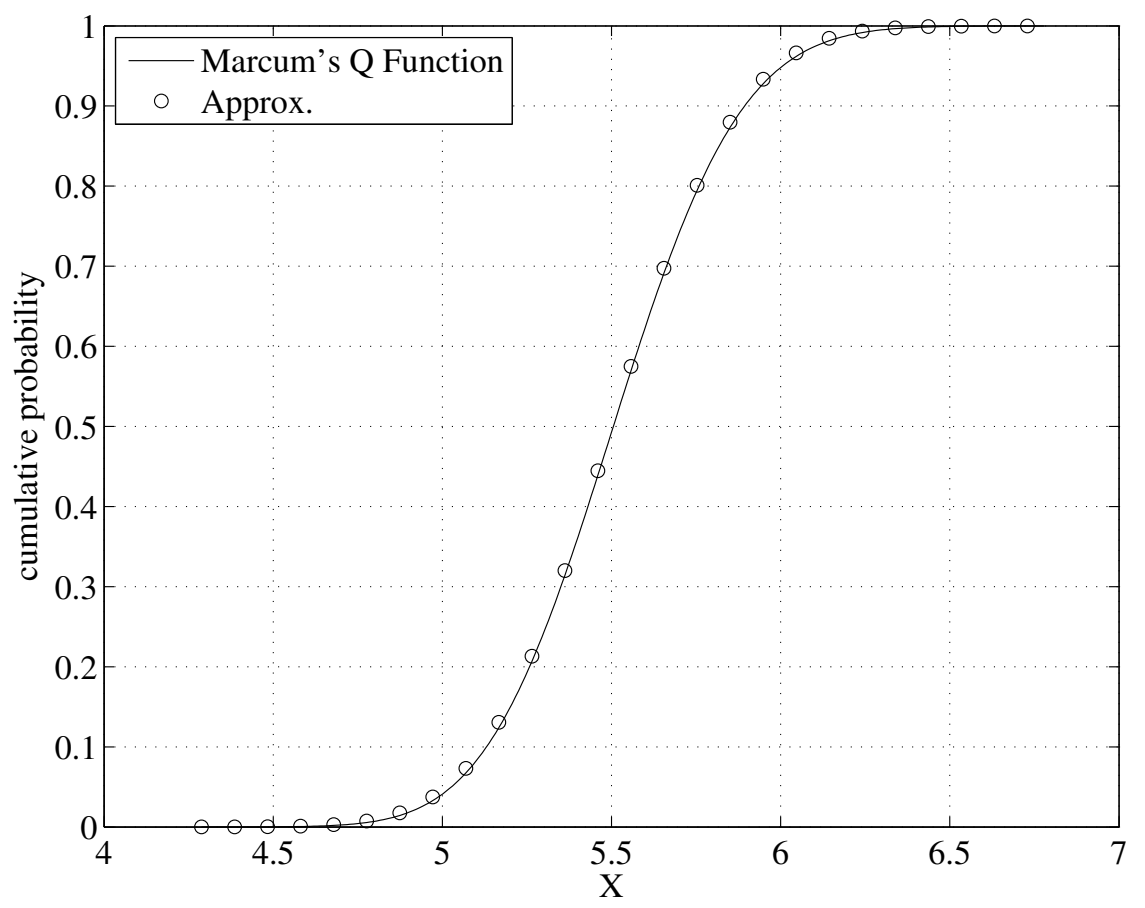

Figure 7.3: True CDF and approximation for $\rho=0.1$.

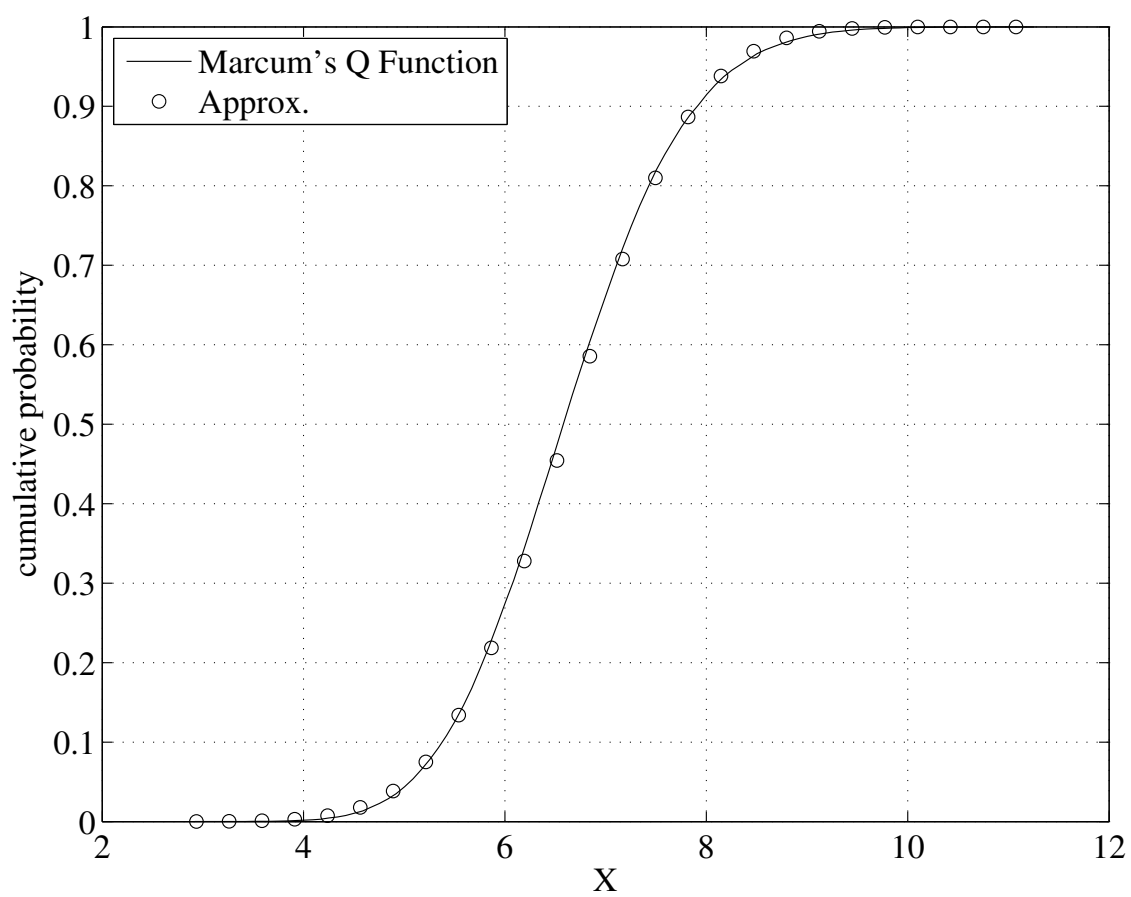

Figure 7.4: True CDF and approximation for $\rho=0.2$. 


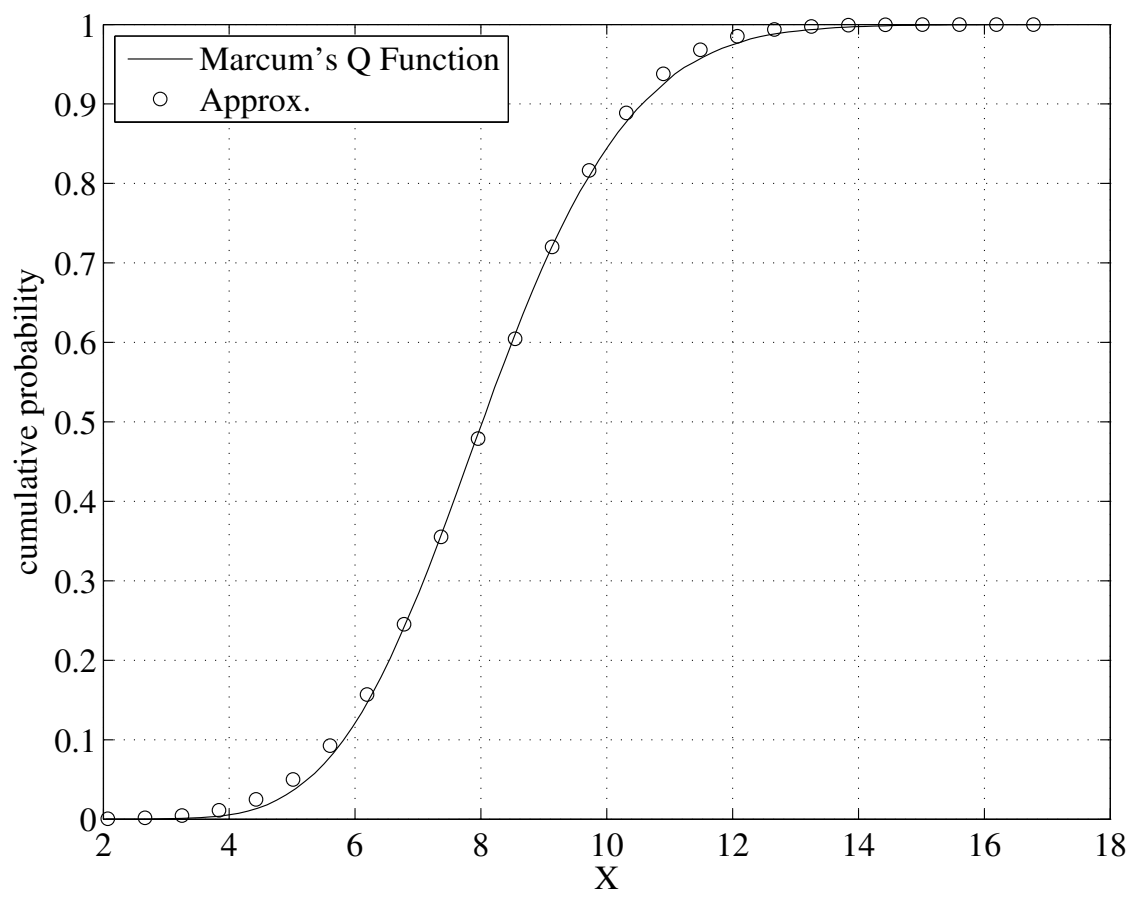

Figure 7.5: True CDF and approximation for $\rho=0.3$.

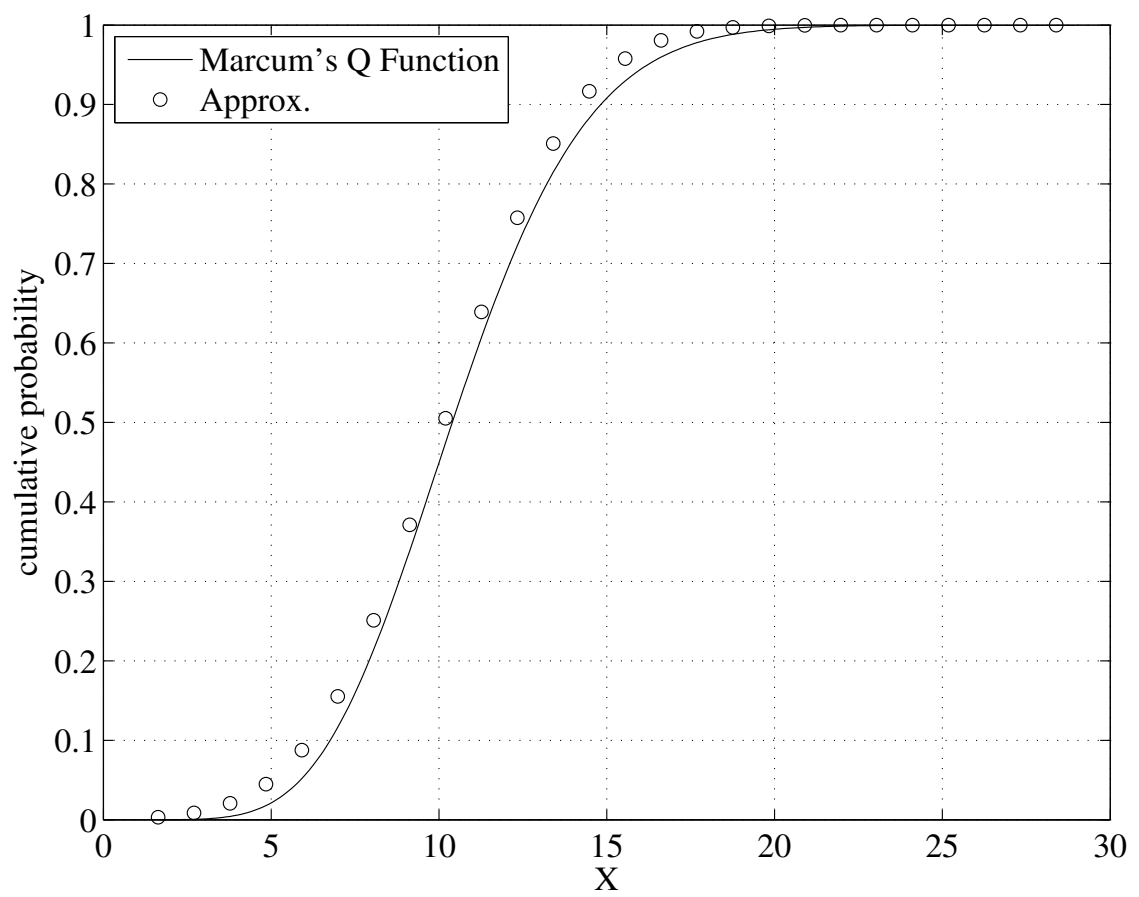

Figure 7.6: True CDF and approximation for $\rho=0.4$. 
as the convex constraint

$$
\frac{\gamma_{\mathrm{s}, \min } \sigma_{\mathrm{s}}^{2}-\operatorname{tr}\left(\tilde{\mathbf{h}} \tilde{\mathbf{h}}^{H} \mathbf{W}\right)}{\operatorname{erfc}^{-1}(2(1-\beta))} \geq 2 \rho\left\|\left(\boldsymbol{\Sigma}_{\mathrm{e}} \tilde{\mathbf{h}} \tilde{\mathbf{h}}^{H}\right)^{\frac{1}{2}} \mathbf{W}\right\|_{2} .
$$

The robust transmit power minimisation problem can therefore be stated as the following SDP

$$
\begin{array}{cl}
\min _{\mathbf{W}} & \operatorname{tr}(\mathbf{W}) \\
\text { s.t. } & (7.23), \quad i=1 \ldots K \\
& (7.24), \\
& \mathbf{W} \succeq 0, \\
& \operatorname{rank}(\mathbf{W})=1 .
\end{array}
$$

Constraints $(7.25 \mathrm{~d})$ and $(7.25 \mathrm{e})$ are included in the above optimisation problem because of the definition of $\mathbf{W}$. Note that constraint $(7.25 \mathrm{e})$ is a non-convex constraint hence, we apply the idea of SDR and relax problem (7.25) by dropping the non-convex rank-one constraint and obtain the following convex robust transmit power minimisation SDP

$$
\begin{array}{ll}
\min _{\mathbf{W}} & \operatorname{tr}(\mathbf{W}) \\
\text { s.t. } & (7.23), \quad i=1 \ldots K \\
& (7.24) \text { and }(7.25 \mathrm{~d}) .
\end{array}
$$

Problem (7.26) is in a convex form and standard interior point methods can be used to solve it efficiently.

After solving (7.26) one needs to recover the optimum beamforming vector, $\mathbf{w}^{*}$, from $\mathbf{W}$. If $\mathbf{W}$ is rank-one, then $\mathbf{w}^{*}$ can be chosen to be the principal eigenvector of $\mathbf{W}$. For the case where $\mathbf{W}$ has rank higher than one, the well known Gaussian randomisation technique [146] can be used to recover a good rank-one approximation. However, in our extensive numerical simulations, we have never obtained a solution that had a rank higher than one. This behaviour is similar to that reported in other beamforming problems $[82,99]$.

\subsubsection{Robust SU Outage Probability Minimisation}

The $\mathrm{SU}_{\mathrm{Rx}}$ outage probability minimisation problem is the robust counterpart of the $\mathrm{SU}_{\mathrm{Rx}} \mathrm{SNR}$ maximisation problem. This problem seeks to minimise the $\mathrm{SU}_{\mathrm{Rx}}$ outage probability while constraining the total $\mathrm{SU}_{\mathrm{Tx}}$ transmit power and the outage 
probability of the $\mathrm{PU}_{\mathrm{Rx}} \mathrm{S}$. Mathematically this problem is stated as

$$
\begin{array}{ll}
\min _{\mathbf{W}} & 1-\frac{1}{2} \operatorname{erfc}\left(\frac{\gamma_{\mathrm{s}, \min } \sigma_{\mathrm{s}}^{2}-\operatorname{tr}\left(\tilde{\mathbf{h}} \tilde{\mathbf{h}}^{H} \mathbf{W}\right)}{2 \rho\left\|\left(\boldsymbol{\Sigma}_{\mathrm{e}} \tilde{\mathbf{h}} \tilde{\mathbf{h}}^{H}\right)^{\frac{1}{2}} \mathbf{W}\right\|_{2}}\right) \\
\text { s.t. } & (7.23), \quad i=1 \ldots K \\
& \operatorname{tr}(\mathbf{W}) \leq P_{T, \max }, \\
& \mathbf{W} \succeq 0, \\
& \operatorname{rank}(\mathbf{W})=1 .
\end{array}
$$

Using the epigraph form and through the application of SDR, the relaxed form of problem (7.27) can be restated as

$$
\begin{array}{ll}
\min _{\mathbf{W}, t} & t \\
\text { s.t. } & \frac{\gamma_{\mathrm{s}, \min } \sigma_{\mathrm{s}}^{2}-\operatorname{tr}\left(\tilde{\mathbf{h}} \tilde{\mathbf{h}}^{H} \mathbf{W}\right)}{\operatorname{erfc}^{-1}(2(1-t))} \geq 2 \rho\left\|\left(\boldsymbol{\Sigma}_{\mathrm{e}} \tilde{\mathbf{h}} \tilde{\mathbf{h}}^{H}\right)^{\frac{1}{2}} \mathbf{W}\right\|_{2} \\
& (7.23), \quad i=1 \ldots K \\
& (7.27 \mathrm{c}) \text { and }(7.27 \mathrm{~d})
\end{array}
$$

where $t$ is an auxiliary variable. Problem (7.28) is a non-convex optimisation problem. However, for any fixed value of $t$ the set of feasible $\mathbf{W}$ is convex and hence the problem is quasi convex. Therefore, for some given $t$, problem (7.28) can be expressed as the following convex feasibility problem

$$
\begin{array}{ll}
\text { find } & \mathbf{W} \\
\text { s.t. } & (7.28 \mathrm{~b}),(7.28 \mathrm{c}),(7.27 \mathrm{c}) \text { and }(7.27 \mathrm{~d}) .
\end{array}
$$

The bisection method [29] can be used to solve problem (7.29) in an iterative manner. Since the rank constraint was relaxed to obtain (7.29), W is not guaranteed to be a rank one matrix. However, in our extensive numerical simulations we have never obtained a solution that had a rank higher than one, therefore, the optimum beamforming vector, $\mathbf{w}^{*}$, is chosen to be the principal eigenvector of $\mathbf{W}$. This behaviour is similar to that reported in other beamforming problems [82, 99].

\subsubsection{Robust Maximum PU Outage Probability Minimisa- tion}

Since $\mathrm{PU}_{\mathrm{Rx}}$ SINR directly influences the $\mathrm{PU}_{\mathrm{Rx}}$ outage probability, minimisation of the maximum $\mathrm{PU}_{\mathrm{Rx}}$ outage probability is an analogous problem to the maximisation of the minimum $\mathrm{PU}_{\mathrm{Rx}} \mathrm{SINR}$ problem. Mathematically this is represented as 
the following relaxed SDP

$$
\begin{array}{ll}
\min _{\mathbf{W}} & \max _{i=1, \ldots, K}\left(1-\frac{P_{\mathrm{p}} \Omega_{c}^{(i)} \exp \left(-\frac{\gamma_{\mathrm{T}}^{(i)} \sigma_{\mathrm{p}}^{2}}{P_{\mathrm{p}} \Omega_{c}^{(i)}}\right)}{\gamma_{\mathrm{T}}^{(i)} \operatorname{tr}\left(\boldsymbol{\Sigma}_{\mathbf{f}_{i}} \mathbf{W}\right)+P_{\mathrm{p}} \Omega_{c}^{(i)}}\right) \\
\text { s.t. } & (7.24) \text { and }(7.25 \mathrm{~d}), \\
& \operatorname{tr}(\mathbf{W}) \leq P_{T, \max .}
\end{array}
$$

It is easily shown that problem (7.30) can be equivalently expressed as

$$
\begin{array}{ll}
\max _{\mathbf{W}} & \min _{i=1, \ldots, K}\left(\frac{P_{\mathrm{p}} \Omega_{c}^{(i)} \exp \left(-\frac{\gamma_{\mathrm{T}}^{(i)} \sigma_{\mathrm{p}}^{2}}{P_{\mathrm{p}} \Omega_{c}^{(i)}}\right)}{\gamma_{\mathrm{T}}^{(i)} \operatorname{tr}\left(\boldsymbol{\Sigma}_{\mathbf{f}_{i}} \mathbf{W}\right)+P_{\mathrm{p}} \Omega_{c}^{(i)}}\right) \\
\text { s.t. } & (7.24),(7.25 \mathrm{~d}) \text { and }(7.30 \mathrm{c}) .
\end{array}
$$

Using the epigraph form, problem (7.31) can be rewritten as

$$
\begin{array}{cl}
\max _{t, \mathbf{W}} & t \\
\text { s.t. } & (1-t)\left(\gamma_{\mathrm{T}}^{(i)} \operatorname{tr}\left(\Sigma_{\mathbf{f}_{i}} \mathbf{W}\right)+P_{\mathrm{p}} \Omega_{c}^{(i)}\right) \leq \\
& P_{\mathrm{p}} \Omega_{c}^{(i)} \exp \left(-\frac{\gamma_{\mathrm{T}}^{(i)} \sigma_{\mathrm{p}}^{2}}{P_{\mathrm{p}} \Omega_{c}^{(i)}}\right), \quad i=1 \ldots K \\
& (7.24),(7.25 \mathrm{~d}) \text { and }(7.30 \mathrm{c}) .
\end{array}
$$

Problem (7.32) is a non-convex optimisation problem. However, for any fixed value of $t$ the set of feasible $\mathbf{W}$ is convex and hence the problem is quasi convex. Therefore, for some given $t$, problem (7.32) can be expressed as the following convex feasibility problem

$$
\begin{array}{ll}
\text { find } & \mathbf{W} \\
\text { s.t. } & (7.24),(7.25 \mathrm{~d}),(7.30 \mathrm{c}) \text { and }(7.32 \mathrm{~b}) .
\end{array}
$$

The bisection method [29] can be used to solve problem (7.33) in an iterative manner. Since the rank constraint was relaxed to obtain (7.33), $\mathbf{W}$ is not guaranteed to be a rank one matrix. However, in our extensive numerical simulations we have never obtained a solution that had a rank higher than one, therefore, the optimum beamforming vector, $\mathbf{w}^{*}$, is chosen to be the principal eigenvector of $\mathbf{W}$. This behaviour is similar to that reported in other beamforming problems [82, 99]. 


\subsection{Simulation Results and Discussion}

We illustrate the performance of our proposed methods through numerical simulations in i.i.d. Rayleigh flat-fading channels. We consider a system with four PUs and one $\mathrm{SU}$ with four transmit antennas, i.e., $K=4$ and $M=4$. In all simulations we have set $P_{\mathrm{p}}=30 \mathrm{dBm}, \gamma_{\mathrm{T}}^{(i)}=10 \mathrm{~dB}, \gamma_{\mathrm{s}, \text { min }}=10 \mathrm{~dB}$ and the noise power at each $\mathrm{PU}$ and $\mathrm{SU}$ receiver is assumed to be $-30 \mathrm{dBm}$, i.e., $\sigma_{\mathrm{p}}^{2}=\sigma_{\mathrm{s}}^{2}=-30 \mathrm{dBm}$. The channel powers of all $\mathrm{PU}_{\mathrm{Tx}}$ to $\mathrm{PU}_{\mathrm{Rx}}$ links are set to $0 \mathrm{~dB}$, i.e., $\Omega_{c}^{(i)}=0 \mathrm{~dB}, \forall i$. The SICR at each $\mathrm{PU}_{\mathrm{Rx}}$ is set to $-20 \mathrm{~dB}$, i.e., the interference channel powers are 20 $\mathrm{dB}$ higher than the wanted signal channel powers. This corresponds to $\Omega_{\mathrm{f}_{i}}^{(j)}=-20$ $\mathrm{dB}, \forall i, j$. The channel powers of all links between the $\mathrm{SU}_{\mathrm{Tx}}$ and the $\mathrm{SU}_{\mathrm{Rx}}$ are set to $-10 \mathrm{~dB}$, i.e., $\Omega_{\mathrm{h}}^{(j)}=-10 \mathrm{~dB}, \forall j$. This set-up represents the scenario where the $\mathrm{PU}$ receivers experience high interference from the SU system and the link between the SU transmitter and receiver is weak. This makes it difficult for the SU system to guarantee the required QoS to the PU system while meeting its own QoS requirements.

The maximum PU outage probability, $\alpha^{(i)}$, in optimisation problems that impose PU outage probability constraints is set to $5 \%$. Similarly, the maximum SU outage probability, $\beta$ in optimisation problems that impose a SU outage probability constraint is also set to $5 \%$. According to CSI error model (7.14), $\boldsymbol{\Sigma}_{\mathrm{e}_{i i}}=$ $\left\|\Omega_{\mathrm{h}}^{1 / 2}\right\|_{2}^{2} / 4=-10 \mathrm{~dB}, \forall i$. To illustrate the impact of CSI errors and the effectiveness of our proposed method, we present simulation results for three different values of $\rho$, namely, $0.1,0.2$, and 0.3 .

Results of our proposed method are compared against the full CSI, worst-case and non-robust designs. The non-robust design treats the $\mathrm{SU}_{\mathrm{Tx}}$ to $\mathrm{SU}_{\mathrm{Rx}}$ channel estimate as perfect and imposes an instantaneous $\mathrm{SU}_{\mathrm{Rx}} \mathrm{SNR}$ constraint. The design of the worst-case beamformer is described in the next 2 paragraphs.

The worst-case beamformer that minimises the $\mathrm{SU}_{\mathrm{Tx}}$ transmit power is designed such that the SINR at the $i$ th $\mathrm{PU}_{\mathrm{Rx}}$ is above the threshold $\gamma_{\mathrm{T}}^{(i)}$ and the SNR at the $\mathrm{SU}_{\mathrm{Rx}}$ is above the threshold $\gamma_{\mathrm{s} \text {,min }}$ for every possible realisation of $c_{i}, \mathbf{f}_{i}$ and $\mathbf{e}$. Since instantaneous realisations of $c_{i}$ and $\mathbf{f}_{i}$ are not available, our worst-case design solves problem (7.6) based on the expected value of (7.5). Note that (7.5) is at its minimum when $\left|c_{i}\right|^{2}=\Omega_{c}^{(i)}-\epsilon_{c}$ and $\left|f_{i}^{(j)}\right|^{2}=\Omega_{\mathrm{f}_{i}}^{(j)}+\epsilon_{f}$, for some appropriately chosen values of $\epsilon_{c}, \epsilon_{f} \geq 0$. The worst-case beamformer ensures this minimum value is always above the threshold $\gamma_{\mathrm{T}}^{(i)}$. Furthermore, we impose a norm bound [238] on e, i.e., $\|\mathbf{e}\|_{2} \leq \epsilon_{e}$ and the worst-case beamformer ensures that the $\mathrm{SNR}$ at the $\mathrm{SU}_{\mathrm{Rx}}$ is above the threshold $\gamma_{\mathrm{s} \text {,min }}$ for every possible realisation of e within this bound. The worst-case $\mathrm{SU}_{\mathrm{Rx}}$ constraint is mathematically represented as

$$
\min _{\|\mathbf{e}\|_{2} \leq \epsilon_{e}}\left|\tilde{\mathbf{h}}^{H} \mathbf{w}+\mathbf{e}^{H} \mathbf{w}\right|^{2} \geq \sigma_{\mathrm{s}}^{2} \gamma_{\mathrm{s}, \min }
$$


Utilising the method used for developing the worst-case MVDR beamformer of Section 3.3.2 (Equation 3.58), the above worst-case constraint can be replaced by its lower bound:

$$
\left|\tilde{\mathbf{h}}^{H} \mathbf{w}\right|-\epsilon_{e}\|\mathbf{w}\|_{2} \geq \sqrt{\sigma_{\mathrm{s}}^{2} \gamma_{\mathbf{s}, \min }} .
$$

The worst-case beamformer that minimises the transmit power can therefore be stated as the following SOCP:

$$
\begin{array}{ll}
\min _{\mathbf{w}} & \|\mathbf{w}\|_{2} \\
\text { s.t. } & \frac{\left(\frac{P_{\mathrm{p}}\left(\Omega_{c}^{(i)}-\epsilon_{c}\right)}{\gamma_{\mathrm{T}}^{(i)}}-\sigma_{\mathrm{p}}^{2}\right)}{\Omega_{\mathrm{f}_{i}}^{(1)}+\epsilon_{f}} \geq\|\mathbf{w}\|_{2}, \quad i=1 \ldots K \\
& \mathbf{h}^{H} \mathbf{w} \geq \epsilon_{e}\|\mathbf{w}\|_{2}+\sigma_{\mathrm{s}} \sqrt{\gamma_{\mathrm{s}, \min } .}
\end{array}
$$

The facts that $\Omega_{\mathrm{f}_{i}}^{(j)}, \forall j$ are equal in this simulation scenario and neither the objective nor the constraints change if the beamforming vector undergoes an arbitrary phase rotation have been used in the derivation of (7.36).

To provide a fair comparison of the worst-case method with the method proposed in this chapter, $\epsilon_{c}, \epsilon_{f}$ and $\epsilon_{e}$ are chosen such that $\operatorname{Pr}\left\{\left|c_{i}\right|^{2} \geq \Omega_{c}^{(i)}-\epsilon_{c}\right\}$ $\prod_{j=1}^{R} \operatorname{Pr}\left\{\left|f_{i}^{(j)}\right|^{2} \leq \Omega_{\mathrm{f}_{i}}^{(j)}+\epsilon_{f}\right\}=1-\alpha^{(i)}, \forall i$ and $\operatorname{Pr}\left\{\|\mathbf{e}\|_{2} \leq \epsilon_{e}\right\}=1-\beta$. This ensures that the probability of encountering a scenario that the worst-case beamformer has not been designed for is the same as the required outage probability of the proposed methods. In our simulations of the worst-case beamformer, e is a zero-mean vector with independently and identically distributed complex Gaussian entries and the variance of each entry equal to $0.1^{2}\left\|\Omega_{\mathrm{h}}^{1 / 2}\right\|_{2}^{2} / 4=-30 \mathrm{~dB}$. This corresponds to the scenario where $\rho=0.1$ in the simulations of our proposed $\mathrm{SU}_{\mathrm{Tx}}$ transmit power minimising robust beamformer.

In Fig. 7.7, the CDF of the SINR at the $\mathrm{PU}_{\mathrm{Rx}} 1$ resulting from solving multiple realisations of the $\mathrm{SU}_{\mathrm{Tx}}$ power minimisation problem is provided. In each realisation of the problem, new instances of the required channels are generated and the beamforming problem is solved to obtain the beamformer weights. This procedure is followed for all results presented in this section. We see that the required probability of the achieved $\mathrm{PU}_{\mathrm{Rx}}$ SINR being below $10 \mathrm{~dB}$ is satisfied by the robust optimisation scheme proposed in this chapter for all three levels of CSI estimate error. We see that increasing the CSI estimate error does not significantly degrade the PU's performance. Being very conservative, the worst-case design corresponding to $\rho=0.1$ achieves a lower outage probability than the proposed robust method for all three levels of CSI estimate error. As a reference, the PU's achievable SINR when the SU doesn't exist is also provided. This has been obtained by assuming that the $\mathrm{PU}_{\mathrm{Rx}}$ is able to perfectly cancel the interference generated by other PUs. 


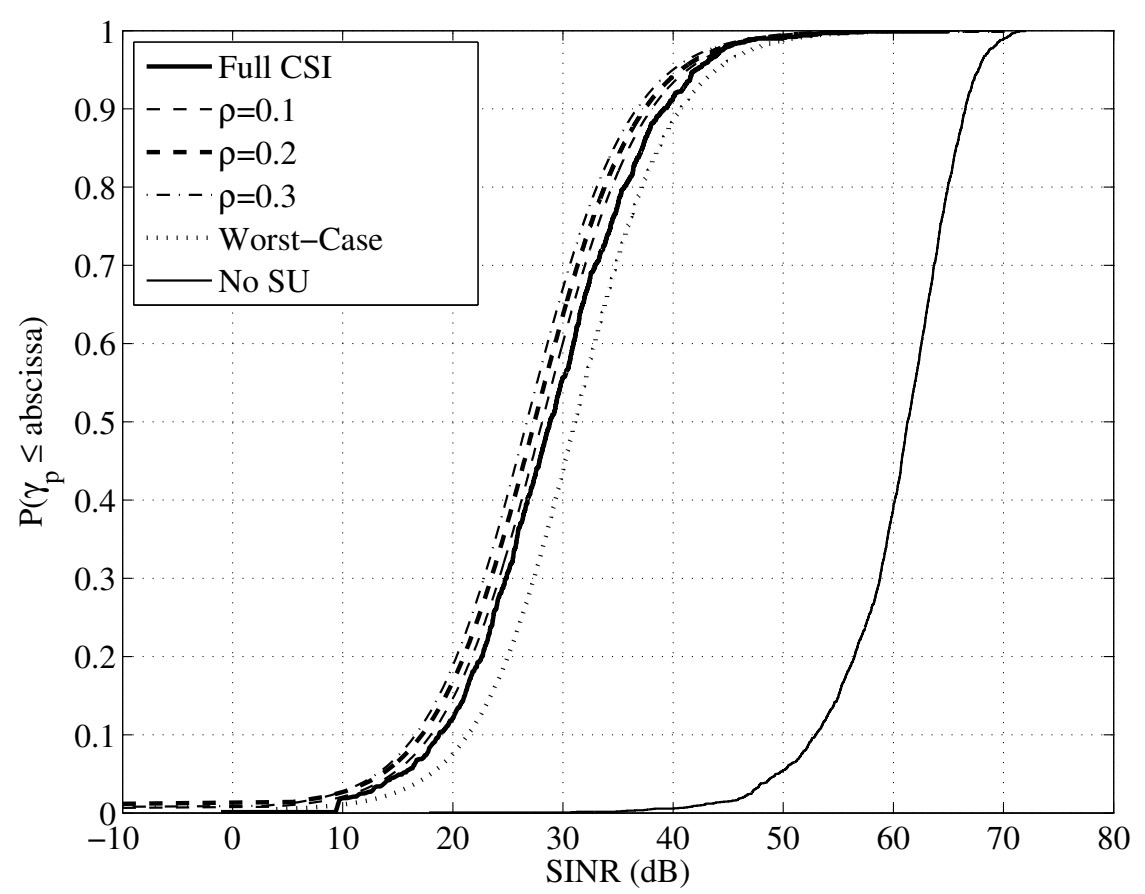

Figure 7.7: CDF of SINR at $\mathrm{PU}_{\mathrm{Rx}} 1$ for the $\mathrm{SU}_{\mathrm{Tx}}$ power minimisation problem.

The performance loss due to the SU's transmission is clearly visible.

The CDF of the $\mathrm{SNR}$ at the $\mathrm{SU}_{\mathrm{Rx}}$ obtained by solving the $\mathrm{SU}_{\mathrm{Tx}}$ power minimisation problem for the proposed robust method along with the full CSI, non-robust and the worst-case designs are shown in Fig. 7.8. We see that the probability of outage for the full CSI design is zero. The full CSI design performance serves as a benchmark for the other methods. The proposed robust beamformer satisfies the outage probability constraint for all three levels of CSI estimate error. We observe that as the level of the channel estimation error increases, the SNR curves move away from the ideal step function response of the full CSI case. The probability that the SNR is below $10 \mathrm{~dB}$ in the worst-case approach is almost zero which implies that very conservative solutions are obtained. Since the non-robust design does not take into consideration the error in the $\mathrm{SU}_{\mathrm{Tx}}$ to $\mathrm{SU}_{\mathrm{Rx}}$ channel estimate, the outage probability of this design is almost $50 \%$. This level of performance is generally unacceptable for most practical systems and highlights the importance of robust designs.

The extremely conservative nature of the worst-case design for the $\mathrm{SU}_{\mathrm{Tx}}$ power minimisation problem is further demonstrated by the SU blocking probabilities listed in Table 7.1. SU blocking probability is defined as the probability that the $\mathrm{SU}$ is not able to access the channel, i.e., the probability that the optimisation problem is infeasible due to either SU or PU QoS constraints not being able to be satisfied. As can be seen in Table 7.1, the worst-case design results in extremely high SU blocking probability which would render it impractical.

In Fig. 7.9, the CDF of the total SU transmit power obtained by solving the 


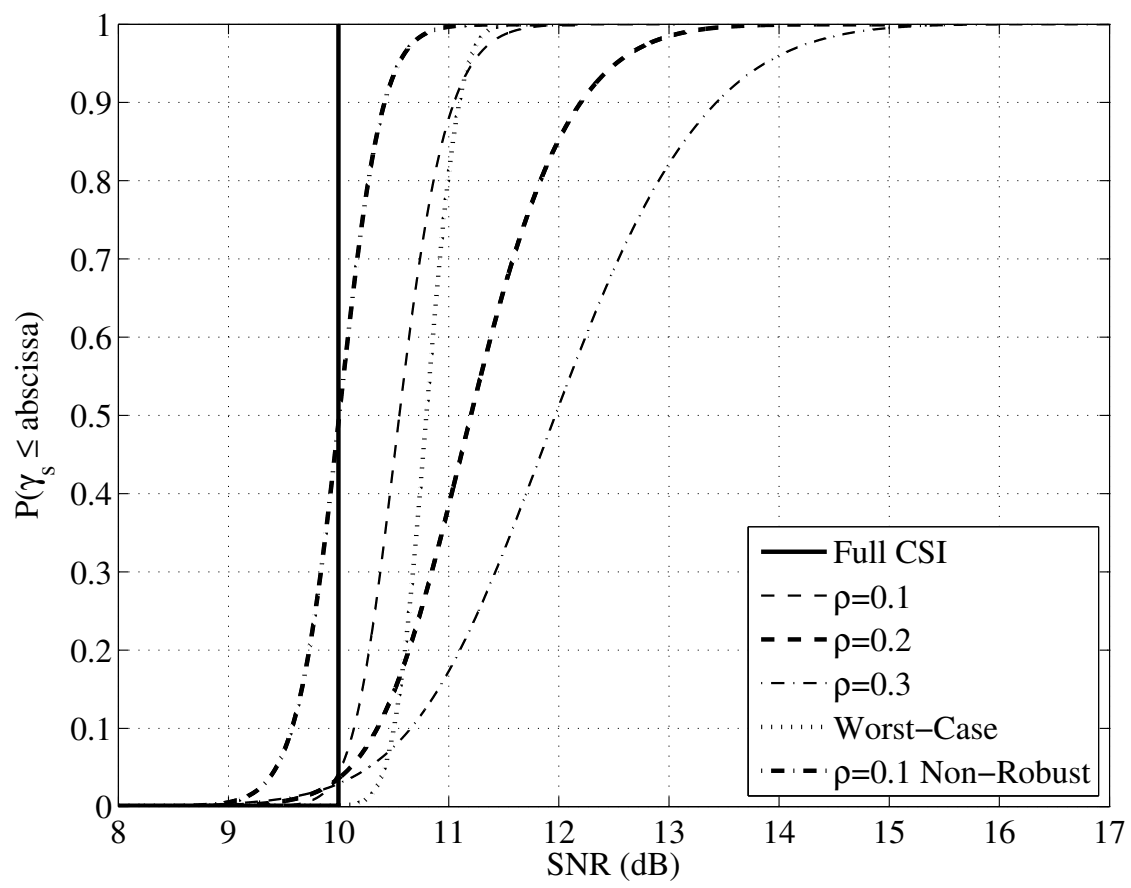

Figure 7.8: CDF of $\mathrm{SNR}$ at the $\mathrm{SU}_{\mathrm{Rx}}$ for the $\mathrm{SU}_{\mathrm{Tx}}$ power minimisation problem.

\begin{tabular}{c|c}
\hline \hline Problem & Blocking Probability (\%) \\
\hline \hline$(7.7)$, Full CSI & 0 \\
\hline$(7.26), \rho=0.1$ & 3.8 \\
\hline$(7.26), \rho=0.2$ & 6.6 \\
\hline$(7.26), \rho=0.3$ & 14.4 \\
\hline Worst-Case & 92.3 \\
\hline \hline
\end{tabular}

Table 7.1: SU Blocking Probabilities for the $\mathrm{SU}_{\mathrm{Tx}}$ Power Minimisation Problem. 


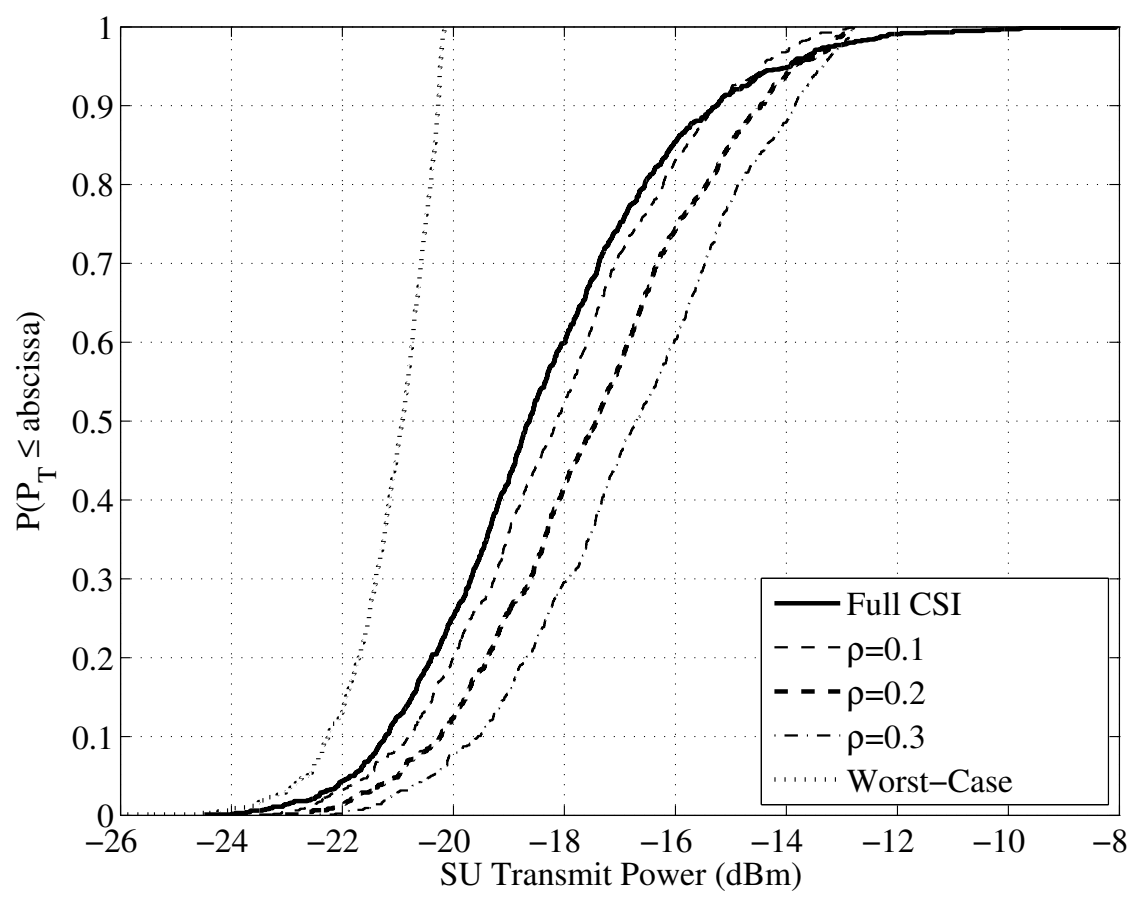

Figure 7.9: $\mathrm{CDF}$ of total $\mathrm{SU}$ transmit power for the $\mathrm{SU}_{\mathrm{Tx}}$ power minimisation problem.

$\mathrm{SU}_{\mathrm{Tx}}$ power minimisation problem is shown. Being very aggressive in protecting the PU, the worst-case design consumes the least amount of transmit power. We see that for the proposed robust design, the power consumption increases as the channel uncertainty increases. This is because the beamformer's beam width is increased to handle the increased channel uncertainty which reduces the amount of signal power that arrives at the $\mathrm{SU}_{\mathrm{Rx}}$. Hence, in order to meet the SU's outage probability requirement, the beamformer compensates for the reduction in the received power by increasing the transmitted power.

Figs. 7.10 and 7.11 compare the CDF of the SINR at $\mathrm{PU}_{\mathrm{Rx}} 1$ and the CDF of the $\mathrm{SNR}$ at the $\mathrm{SU}_{\mathrm{Rx}}$ obtained by solving the robust $\mathrm{SU}_{\mathrm{Tx}}$ power minimisation problem for two $\mathrm{PU}_{\mathrm{Rx}}$ outage probabilities, respectively. The CDFs shown are for $\mathrm{PU}_{\mathrm{Rx}}$ outage probabilities of $1 \%$ and $5 \%$, while $\rho=0.1$ and the $\mathrm{SU}_{\mathrm{Rx}}$ outage probability is set to $5 \%$ in both cases. We see that the required probability of the achieved $\mathrm{PU}_{\mathrm{Rx}}$ SINR being below $10 \mathrm{~dB}$ is satisfied for both cases. Similarly, the $\mathrm{SU}_{\mathrm{Rx}}$ outage probability requirement is also satisfied for both cases. As expected, the performance of the $\mathrm{PU}_{\mathrm{Rx}}$ improves when the $\mathrm{PU}_{\mathrm{Rx}}$ outage probability is reduced from $5 \%$ to $1 \%$. This is due to the reduction in the allowed level of $\mathrm{SU}$ interference at the PU. We also see that the $\mathrm{SU}_{\mathrm{Rx}} \mathrm{SNR}$ curve moves towards the ideal step function response of the full CSI case (see Fig. 7.8) when the $\mathrm{PU}_{\mathrm{Rx}}$ outage probability is reduced from $5 \%$ to $1 \%$. This is because the $\mathrm{SU}_{\mathrm{Tx}}$ has to reduce its transmitted power in order to satisfy the lower $\mathrm{PU}_{\mathrm{Rx}}$ outage probability requirement. Furthermore, we have observed that the SU blocking probability in- 


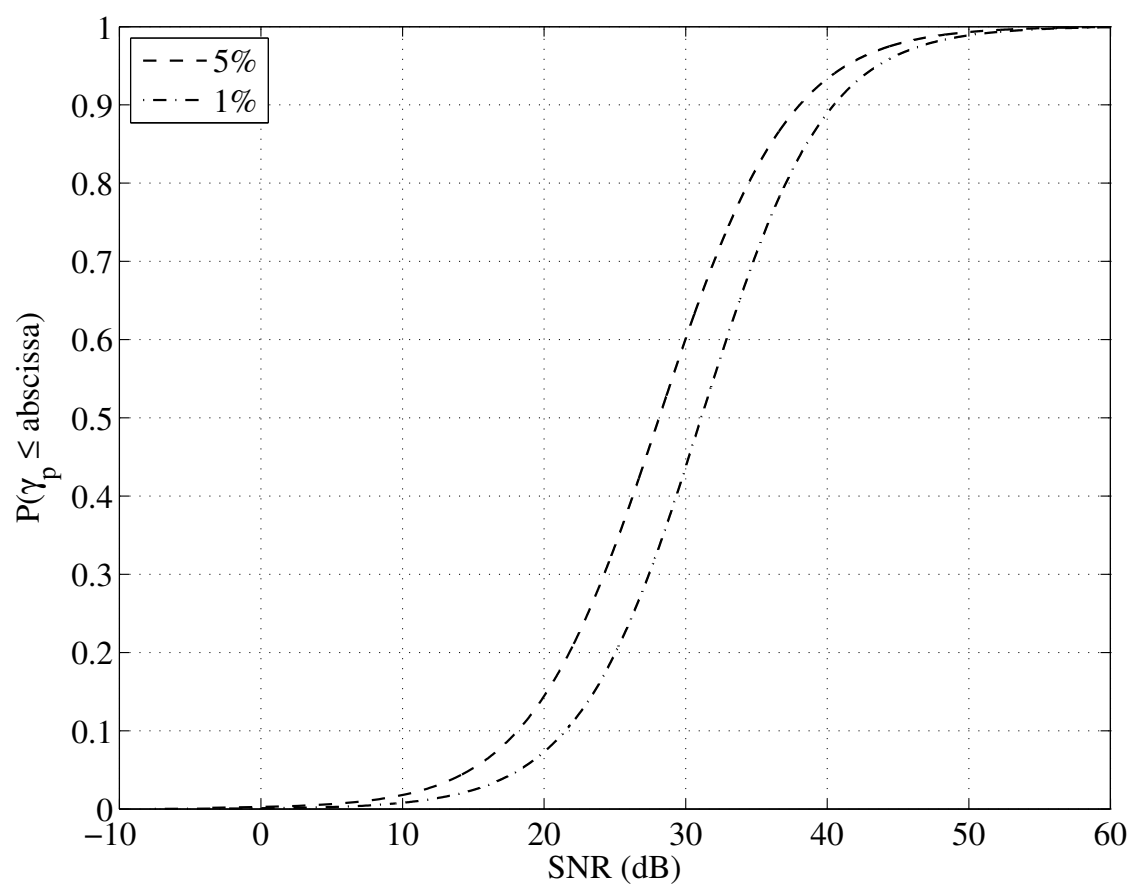

Figure 7.10: $\mathrm{CDF}$ of $\mathrm{SINR}$ at $\mathrm{PU}_{\mathrm{Rx}} 1$ for $\alpha^{(1)}=1 \%$ and $5 \%$ for the $\mathrm{SU}_{\mathrm{Tx}}$ power minimisation problem. $\rho=0.1$ and $\beta=5 \%$ in both cases.

creases from $3.8 \%$ to $40 \%$ when the $\mathrm{PU}_{\mathrm{Rx}}$ outage probability is reduced from $5 \%$ to $1 \%$. This is because the reduced $\mathrm{PU}_{\mathrm{Rx}}$ outage probability causes the $\mathrm{SU}_{\mathrm{Tx}}$ to reduce its transmitted power thus making it harder for the $\mathrm{SU}_{\mathrm{Rx}}$ outage probability requirement to be satisfied. This reduction in the $\mathrm{SU}_{\mathrm{Tx}}$ 's transmitted power can be clearly seen in Fig. 7.12.

Fig. 7.13 shows the CDF of the SINR at the $\mathrm{PU}_{\mathrm{Rx}} 1$ obtained by solving the $\mathrm{SU}_{\mathrm{Rx}}$ outage probability minimisation problem. We see that the required outage probability of $5 \%$ is satisfied for all three levels of CSI estimate error. The CDF of the attainable $\mathrm{SU}_{\mathrm{Rx}}$ outage probability in the simulated channel is shown in Fig. 7.14. This CDF is obtained by solving multiple instances of the $\mathrm{SU}_{\mathrm{Rx}}$ outage probability minimisation problem. In each realisation of the problem, new instances of the required channels are generated and the beamforming problem is solved to obtain the beamformer weights. The attained outage probability of each solution is calculated using the generalised Marcum's Q function [179]. It can be seen that the probability of achieving a certain outage probability decreases as the level of the CSI estimate error increases. This is particularly pronounced for outage probabilities close to zero; for instance, the probability of no outage is approximately $97 \%$ for $\rho=0.1,62 \%$ for $\rho=0.2$ and $15 \%$ for $\rho=0.3$.

In Fig. 7.15, the CDF of the attainable outage probability at $\mathrm{PU}_{\mathrm{Rx}} 1$ resulting from solving the maximum $\mathrm{PU}_{\mathrm{Rx}}$ outage probability minimisation problem is shown. This CDF is obtained by solving multiple instances of the $\mathrm{PU}_{\mathrm{Rx}}$ outage probability minimisation problem. In each realisation of the problem, new instances 


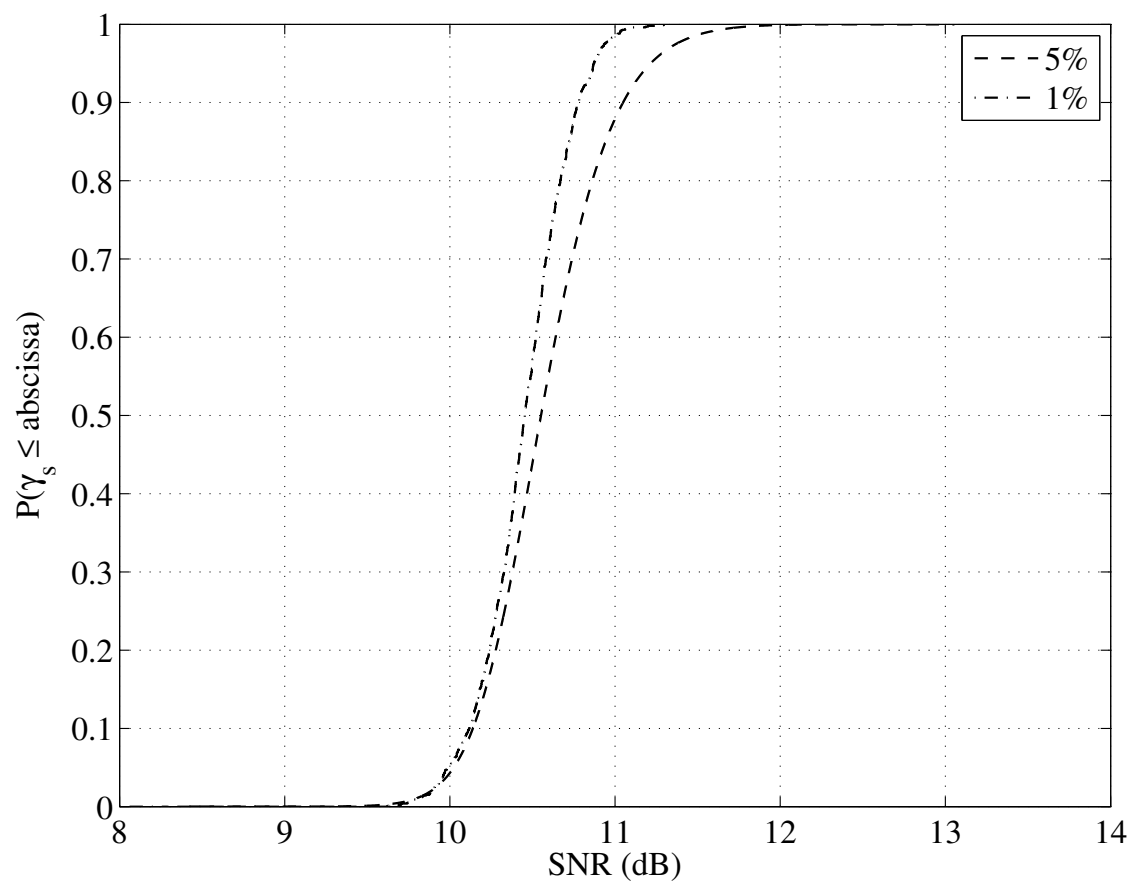

Figure 7.11: $\mathrm{CDF}$ of $\mathrm{SNR}$ at the $\mathrm{SU}_{\mathrm{Rx}}$ for $\alpha^{(1)}=1 \%$ and $5 \%$ for the $\mathrm{SU}_{\mathrm{Tx}}$ power minimisation problem. $\rho=0.1$ and $\beta=5 \%$ in both cases.

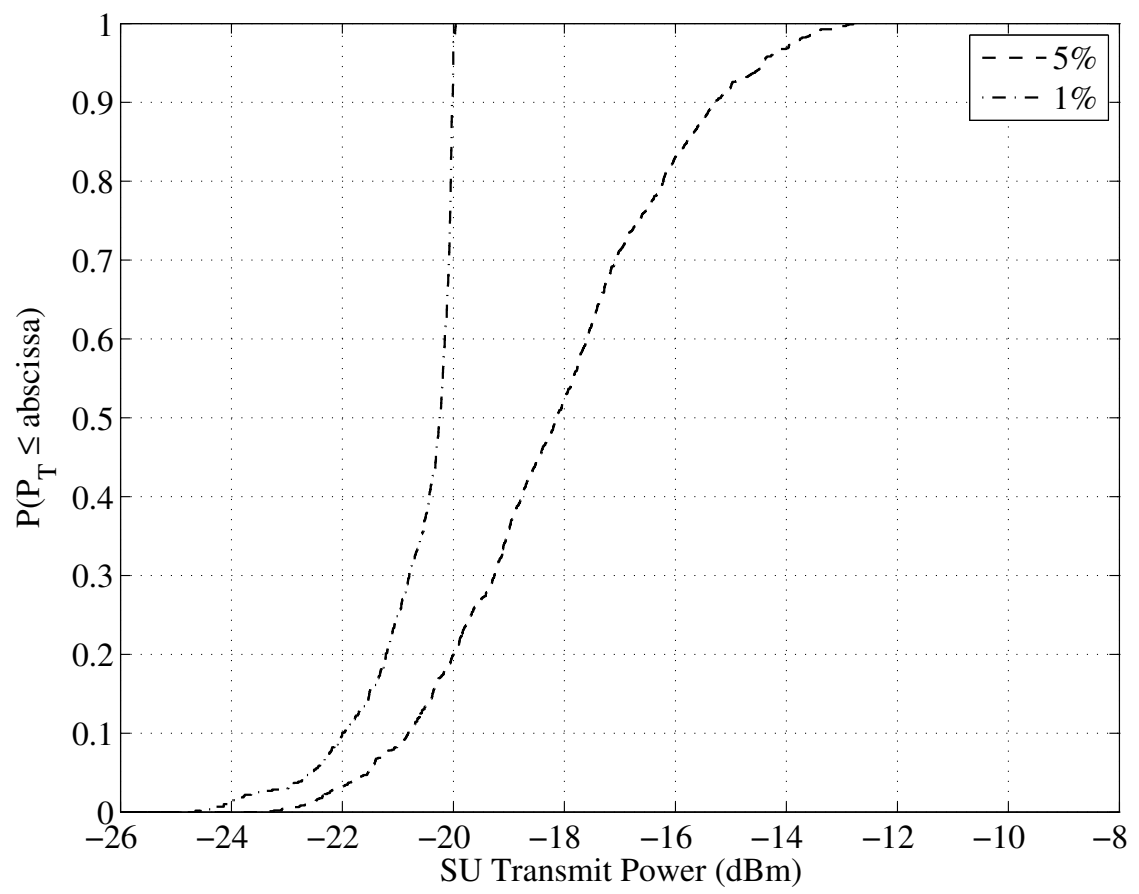

Figure 7.12: CDF of the $\mathrm{SU}_{\mathrm{Tx}}$ transmit power for $\alpha^{(1)}=1 \%$ and $5 \%$ for the $\mathrm{SU}_{\mathrm{Tx}}$ power minimisation problem. $\rho=0.1$ and $\beta=5 \%$ in both cases. 


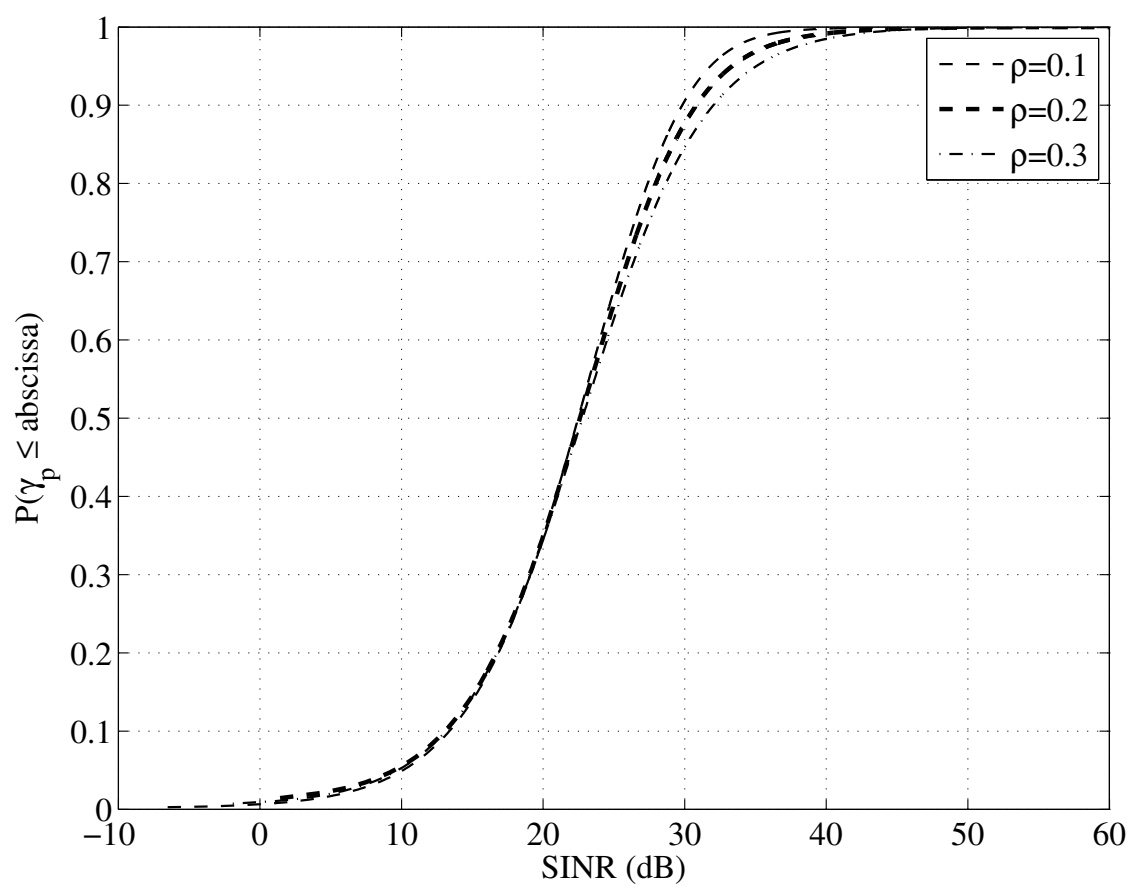

Figure 7.13: CDF of SINR at $\mathrm{PU}_{\mathrm{Rx}} 1$ for the $\mathrm{SU}_{\mathrm{Rx}}$ outage probability minimisation problem.

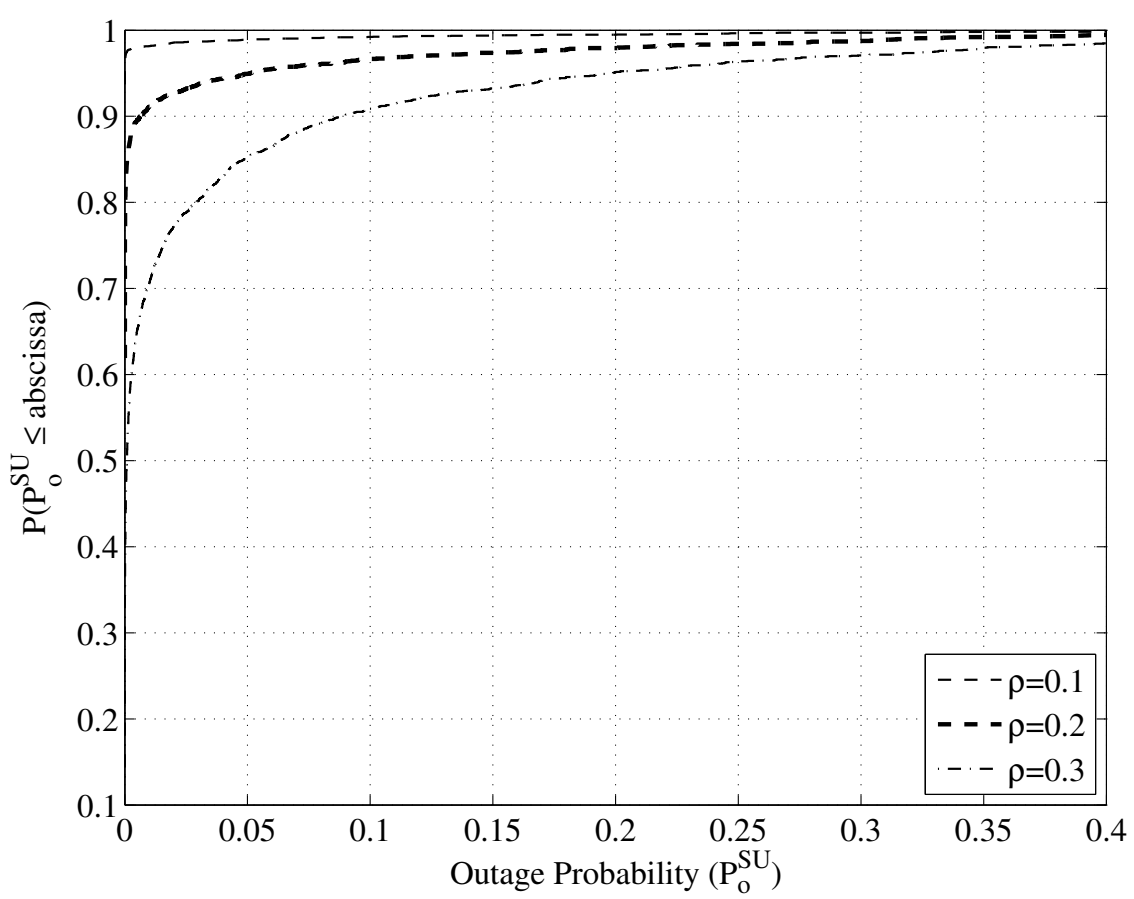

Figure 7.14: $\mathrm{CDF}$ of the $\mathrm{SU}_{\mathrm{Rx}}$ outage probability for the $\mathrm{SU}_{\mathrm{Rx}}$ outage probability minimisation problem. 


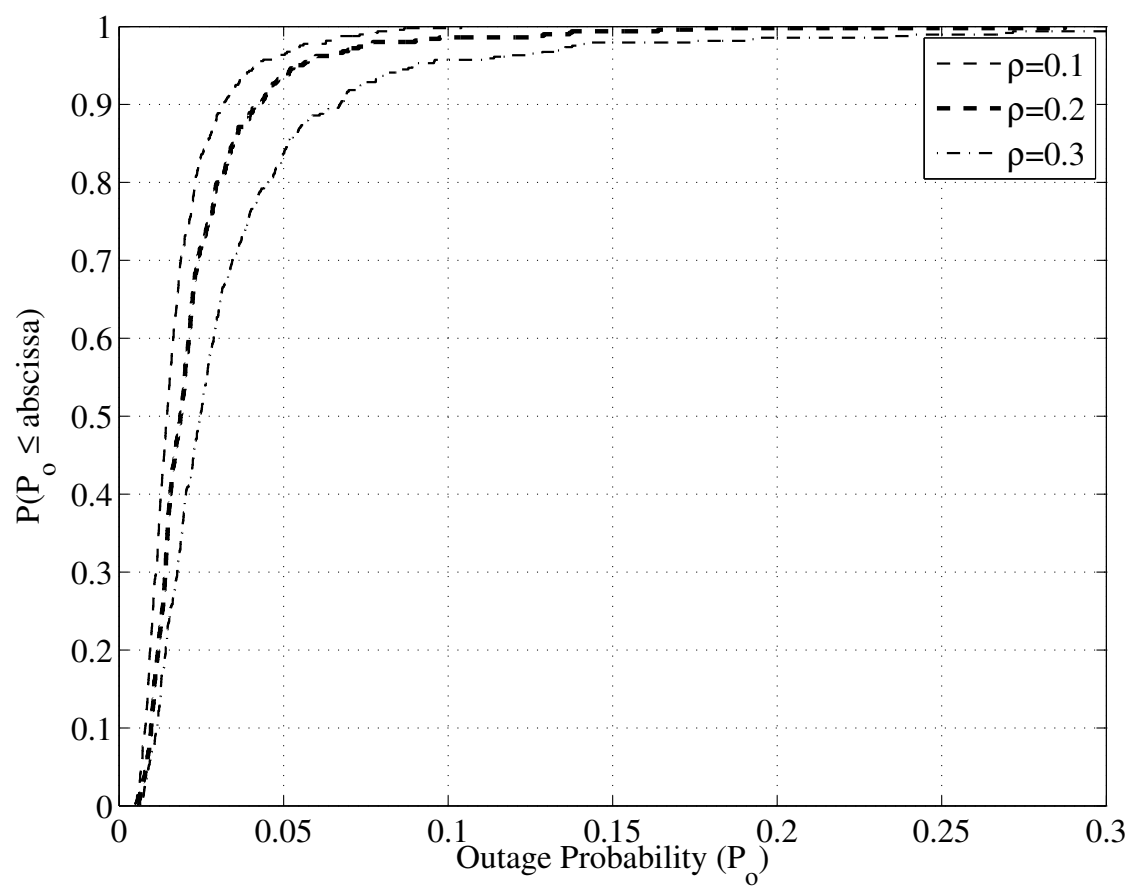

Figure 7.15: $\mathrm{CDF}$ of the $\mathrm{PU}_{\mathrm{Rx}} 1$ outage probability for the maximum $\mathrm{PU}$ outage probability minimisation problem.

of the required channels are generated and the beamforming problem is solved to obtain the beamformer weights. The attained outage probability of each solution is calculated using (7.19). It can be seen that the probability of achieving a certain outage probability decreases as the level of the CSI estimate error increases. This is because the beamformer increases its transmit power to deal with increased channel uncertainty (see Fig. 7.17), which in turn generates more interference at the $\mathrm{PU}$ and thereby increases the probability of outage at the $\mathrm{PU}_{\mathrm{Rx}}$.

The CDF of the SINR at the $\mathrm{SU}_{\mathrm{Rx}}$ obtained by solving the maximum $\mathrm{PU}_{\mathrm{Rx}}$ outage probability minimisation problem is given in Fig. 7.17. It can be seen that the proposed robust beamformer satisfies the outage probability constraint for all three levels of CSI estimate error.

\subsection{Summary}

This chapter has considered the problem of beamforming in an underlay CR network. Under the assumption of perfect CSI, three beamforming problems have been considered, namely i) the $\mathrm{SU}_{\mathrm{Tx}}$ power minimisation problem with $\mathrm{SU}_{\mathrm{Rx}}$ and $\mathrm{PU}_{\mathrm{Rx}}$ SINR constraints; ii) the $\mathrm{SU}_{\mathrm{Rx}}$ SINR maximisation problem with $\mathrm{SU}_{\mathrm{Tx}}$ power and $\mathrm{PU}_{\mathrm{Rx}}$ SINR constraints; and iii) the minimum $\mathrm{PU}_{\mathrm{Rx}}$ SINR maximisation problem with $\mathrm{SU}_{\mathrm{Tx}}$ power and $\mathrm{SU}_{\mathrm{Rx}} \mathrm{SINR}$ constraints. It has been shown that the $\mathrm{SU}_{\mathrm{Tx}}$ power minimisation and the $\mathrm{SU}_{\mathrm{Rx}}$ SINR maximisation problems can be transformed into convex SOCPs and the minimum $\mathrm{PU}_{\mathrm{Rx}}$ SINR maximisation problem can be 


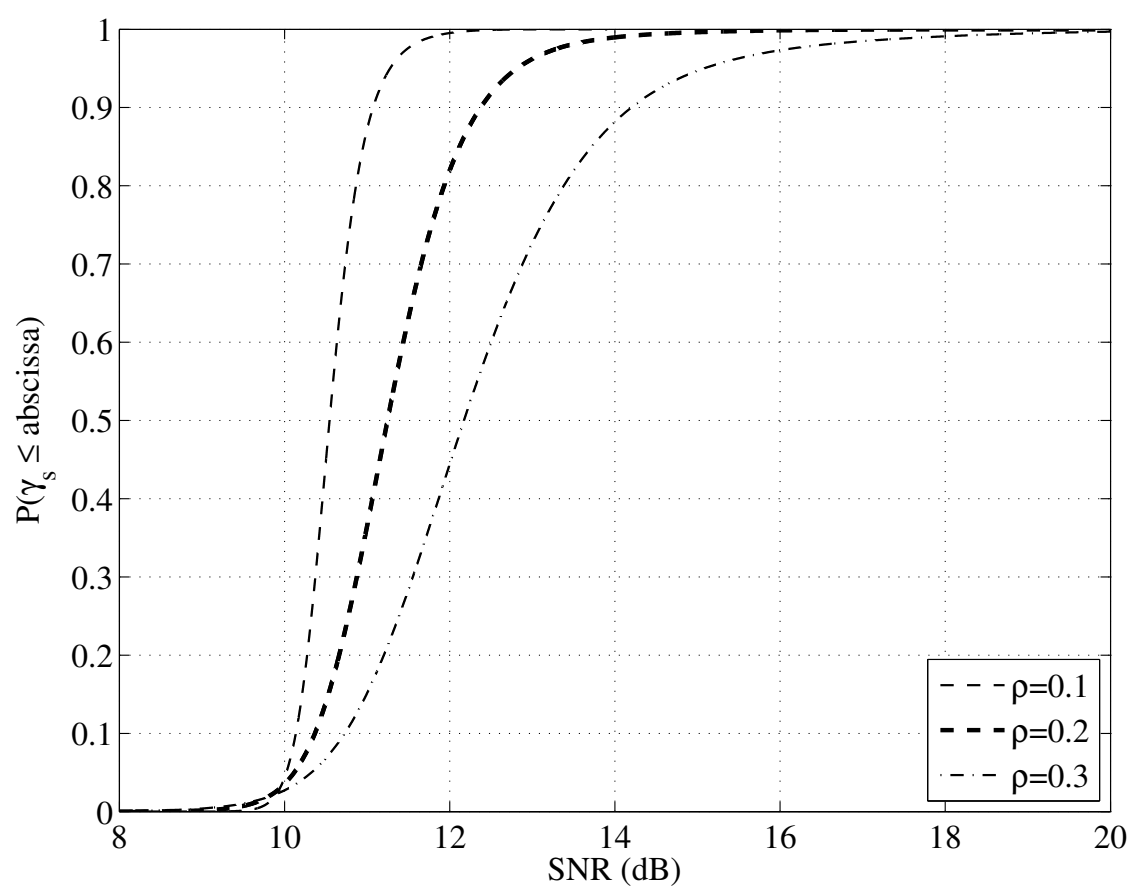

Figure 7.16: $\mathrm{CDF}$ of $\mathrm{SNR}$ at the $\mathrm{SU}_{\mathrm{Rx}}$ for the maximum PU outage probability minimisation problem.

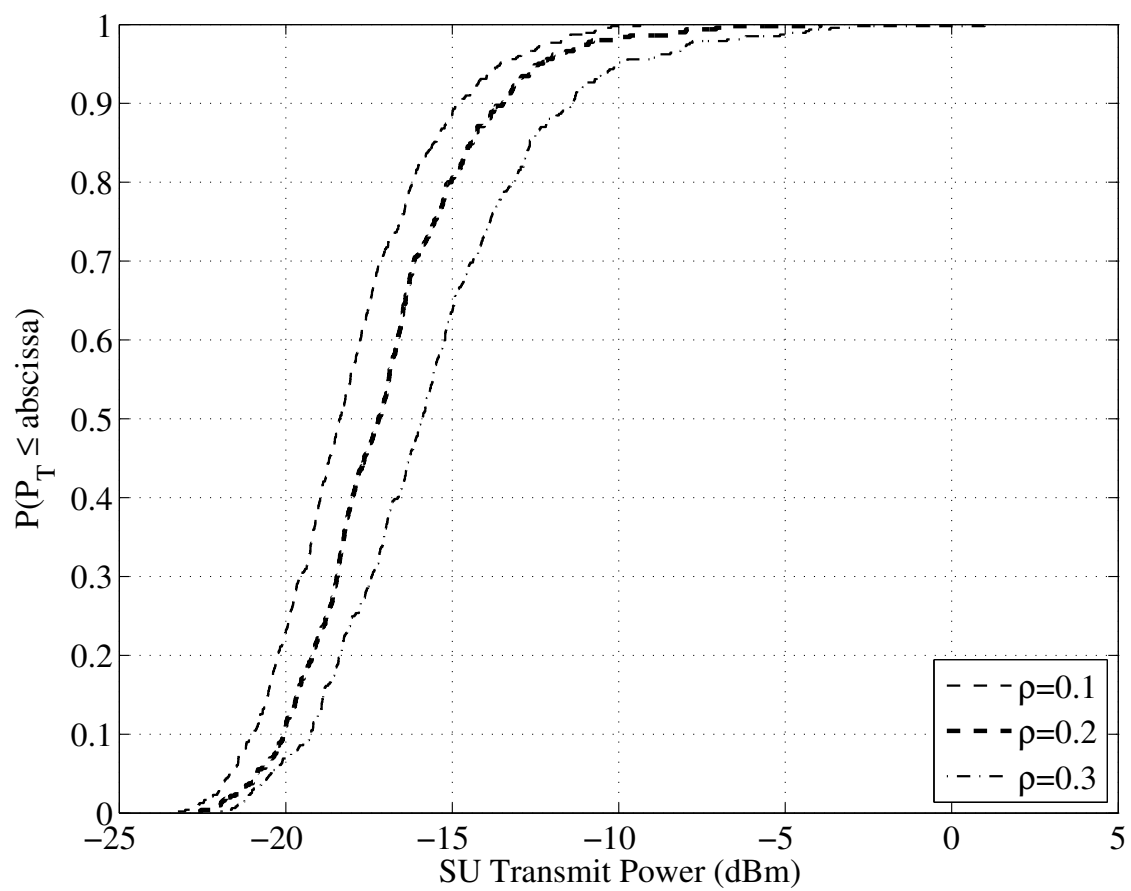

Figure 7.17: CDF of total SU transmit power for the maximum PU outage probability minimisation problem. 
transformed into a convex feasibility problem with SOC constraints.

Under the assumption of partial and imperfect CSI, $\mathrm{SU}_{\mathrm{Rx}}$ and $\mathrm{PU}_{\mathrm{Rx}}$ outage probability expressions have been derived which has led to the development of new robust beamformers. It was demonstrated that the robust $\mathrm{SU}_{\mathrm{Tx}}$ power minimisation problem can be transformed into a convex SDP. It has been shown that the robust $\mathrm{SU}_{\mathrm{Rx}}$ outage probability minimisation and the maximum $\mathrm{PU}_{\mathrm{Rx}}$ outage probability minimisation problems can be transformed into convex feasibility problems.

The performance of the proposed robust beamformers have been demonstrated through the CDFs of the $\mathrm{PU}_{\mathrm{Rx}} \mathrm{SINR}$, the $\mathrm{SU}_{\mathrm{Rx}} \mathrm{SNR}$ and the transmit power and the outage probability at the $\mathrm{PU}_{\mathrm{Rx}}$ and $\mathrm{SU}_{\mathrm{Rx}}$. Our results reveal that the robust beamformers meet the required performance targets for various levels of channel uncertainty. It was also seen that the $\mathrm{SU}_{\mathrm{Tx}}$ power increases with increasing levels of channel uncertainty. 


\section{Chapter 8}

\section{Conclusions and Further Research}

In this chapter the conclusions of this research are summarised, the presented work is critiqued, and further research directions are outlined.

\subsection{Conclusions}

The advent of ubiquitous mobile computing has resulted in an exponential increase in mobile data traffic. Industry projections [47] show that mobile data traffic will continue to grow at the same rate in the foreseeable future. Most countries are facing a looming spectrum crisis whereby customer demand is threatening to outstrip the capacity of the available spectrum. It has been identified that this crisis is largely due to inefficiencies in spectrum access rather than the physical scarcity of the spectrum $[48,60,112,130,158,159,220]$.

CR technology, with its ability to exploit underutilised spectral resources by reusing unused spectrum in a dynamic and opportunistic manner, has been proposed as a viable solution for the efficient use of the radio spectrum [163, 164]. CRs are able to access spectrum resources either on a mutually exclusive basis or concurrently with the PUs. Spectrum sharing or concurrent access has the potential to significantly degrade the PU's performance if interference generated by the SUs is not appropriately managed. Through an appropriately formulated optimisation problem, the transmission parameters of the SUs can be designed to achieve the goals of the CR system while guaranteeing QoS to the PUs.

The problem of spectrum sharing in underlay CR systems has been considered in detail in this thesis. A particular focus has been on the robust formulation of these problems in convex form. In practical wireless communication systems, the assumption of perfect CSI for all links may be over idealistic as it is rarely available. Hence, all of the spectrum sharing optimisation problems proposed in this thesis have sought to provide robustness against partial and imperfect CSI. Convex optimisation has recently been recognised as a powerful tool by the signal processing community. The main advantage of a convex formulation of a problem is 
that the optimisation problem has only one minimum, which is the global minimum. Hence, convex optimisation problems can always be solved, either analytically or numerically, to obtain the optimum solution.

Chapter 2 provided a review of convex optimisation theory and techniques. The concepts of convex sets, convex functions and convex optimisation problems were introduced. Lagrange duality and KKT conditions for optimality were reviewed. A powerful technique known as convex relaxation, which allows non-convex problems to be relaxed into convex problems was presented. Algorithms for solving convex optimisation problems were discussed.

Chapter 3 discussed robust optimisation techniques. Robust optimisation is generally used when there is some degree of uncertainty in the input data. The bounded uncertainty based optimisation and stochastic optimisation was reviewed. Application of these methods to communication problems, specifically, power control, conventional receive and transmit beamforming and cooperative relay beamforming were presented.

The cognitive radio concept and dynamic spectrum access techniques were reviewed in Chapter 4. The state of art spectrum sensing algorithms and spectrum sharing methods were reviewed.

The SU power allocation problem in an underlay CR system was formulated as a GP in Chapter 5. The effect of the PU's transmission was included in the formulations and the problems were studied in both high and low SINR scenarios. It was demonstrated that considering the system sum rate in the optimisation problem, in some circumstances, resulted in improved PU performance without a significant penalty in the SU's sum rate. Optimisation strategies for different channel conditions were presented. A novel method of detecting and removing infeasible SU's QoS constraints from the SU power allocation problem was proposed. Application of this method was shown to result in considerably improved SU performance. Robust SU power allocation problems under CSI uncertainties by considering a PU outage probability constraint were proposed. The results obtained in this chapter quantify the importance of $\mathrm{PU}_{\mathrm{Tx}}$ to $\mathrm{PU}_{\mathrm{Rx}}$ and $\mathrm{SU}_{\mathrm{Tx}} \mathrm{S}$ to $\mathrm{PU} \mathrm{Ux}_{\mathrm{Rx}} \mathrm{CSI}$ and large $\mathrm{SU}$ performance losses are expected if accurate CSI of these links are not available.

In Chapter 6, two cooperative beamformer problems for an underlay CR relay network were formulated. The first problem minimised the total relay transmit power subject to $\mathrm{PU}_{\mathrm{Rx}}$ and $\mathrm{SU}_{\mathrm{Rx}}$ QoS constraints. It was shown that this problem can be cast into a convex SOCP and solved efficiently using interior point methods. In the second problem, the $\mathrm{SU}_{\mathrm{Rx}}$ SINR was maximised subject to $\mathrm{PU}_{\mathrm{Rx}}$ QoS and individual relay transmit power constraints. It was demonstrated that this problem can be solved using three methods, namely, a convex feasibility SOCP, a convex feasibility SDP and a SDP that has the form of a linear-fractional program. The linear-fractional program formulation does not require an iterative procedure for 
solving it, hence, it is the most efficient formulation among the three proposed methods. New robust counterparts of the cooperative beamformer problems that guarantee a certain $\mathrm{PU}_{\mathrm{Rx}}$ outage probability under the assumption of partial and imperfect CSI were developed. These problems have the same structure as those formulated under the assumption of perfect CSI, and hence the same methods can be used to solve the robust formulations. Simulation results have shown how the achieved robustness varies with CSI uncertainty.

Three beamforming problems for an underlay CR network under the assumption of perfect CSI were studied in Chapter 7 . These were the $\mathrm{SU}_{\mathrm{Tx}}$ power minimisation problem with $\mathrm{SU}_{\mathrm{Rx}}$ and $\mathrm{PU}_{\mathrm{Rx}}$ SINR constraints, the $\mathrm{SU}_{\mathrm{Rx}}$ SINR maximisation problem with $\mathrm{SU}_{\mathrm{Tx}}$ power and $\mathrm{PU}_{\mathrm{Rx}}$ SINR constraints and the minimum $\mathrm{PU}_{\mathrm{Rx}}$ SINR maximisation problem with $\mathrm{SU}_{\mathrm{Tx}}$ power and $\mathrm{SU}_{\mathrm{Rx}}$ SINR constraints. It was shown that the $\mathrm{SU}_{\mathrm{Tx}}$ power minimisation and the $\mathrm{SU}_{\mathrm{Rx}} \mathrm{SINR}$ maximisation problems can be transformed into convex SOCPs and the minimum $\mathrm{PU}_{\mathrm{Rx}}$ SINR maximisation problem can be transformed into a convex feasibility problem with SOC constraints. Under the assumption of partial and imperfect CSI, new robust beamformers were developed. It was demonstrated that the robust $\mathrm{SU}_{\mathrm{Tx}}$ power minimisation problem can be transformed into a convex SDP. It was also shown that the robust $\mathrm{SU}_{\mathrm{Rx}}$ outage probability minimisation and the maximum $\mathrm{PU}_{\mathrm{Rx}}$ outage probability minimisation problems can be transformed into convex feasibility problems. The performance of the proposed robust beamformers was demonstrated through the CDFs of the SINR, the transmit power and the outage probability. Our results revealed that the robust beamformers meet the required performance targets for various levels of channel uncertainty.

\subsection{Discussion}

This section reflects on the application of the research presented in this thesis, particularly in the context of wireless system design.

The performance of wireless communication systems can be measured using a number of metrics, two of which include the data rate and the bit error rate (BER). Both the data rate and the BER are functions of either the SNR or the SINR. In a single user system, it suffices to optimise the system SNR or SINR as this directly determines the resulting data rate or BER. In a multi-user system, the definition of a global performance measure is not clear, since each user has their individual performance requirements. However, if a network-centric approach is taken then the system sum rate can be used as a global measure of the system-wide efficiency. While optimising the sum rate achieves the system-wide goal, individual users' QoS requirements also need to be satisfied. Hence, an optimisation problem that optimises a global performance metric must also include constraints for the 
minimum QoS requirements of individual users. Such a constrained optimisation problem represents the tradeoff between user-centric constraints and some networkcentric objective [46].

Chapter 5 considered the power allocation problem for a multi-user cognitive radio network. Hence, a network-centric approach was taken and the sum rate was maximised subject to user-centric constraints. This approach achieved the system-wide goal of maximising the system data throughput and also ensured that the individual users' demands were met. Although a network-centric approach is reasonable, it is also possible to tackle the problem in a user-centric manner. For instance, one could maximise the QoS metric of a user in the highest QoS class, or maximise the QoS metric of the user with the minimum QoS metric (as was done in Section 7.4.3). The user-centric approaches would provide valuable results for comparison against our proposed methods.

The interference from other SU's was treated as noise in the problems formulated in Chapter 5. While this is a valid approach, it does not give the full benefits. Instead of ignoring the information contained in the interference signal, it could be utilised to further improve the SU's performance. The proposed methods would thus benefit from the application of interference cancellation [50,197]. A joint interference cancellation and power control scheme could be explored to evaluate the achievable performance improvements.

Chapter 6 considered a single destination SU cognitive relay network. Since there was only one destination SU receiver, the SINR at the destination receiver was the most meaningful metric to optimise as any other metric is a function of it. The robust beamformers were designed to be robust against CSI uncertainty of the $\mathrm{PU}_{\mathrm{Tx}}$ to $\mathrm{PU}_{\mathrm{Tx}}$ and $\mathrm{SU}_{\mathrm{Rl}}$ to $\mathrm{PU} \mathrm{Rx}_{\mathrm{Rx}}$ links. CSI of these links would be the most difficult to acquire in a practical cognitive radio network and as a starting point, it is reasonable to focus on these links. However, the other links in the system are not immune against CSI uncertainty and the assumption of perfect CSI on these links may not be very practical. Hence, the proposed robust beamformer could be improved by considering uncertainty on other links.

The problems posed in Chapters 5 and 6 have been solved using a centralised algorithm, i.e., a central processor is required to acquire CSI for all links, solve the optimisation problems and distribute the resulting control parameters to all transmitting nodes in the CR network. A control channel is needed to facilitate the exchange of the required information. The bandwidth requirements for this control channel will grow as the network size increases and may become prohibitive for practical implementation. Distributed methods $[46,75]$ which minimise the amount of information exchanged to solve the proposed optimisation problems would be preferred.

Due to the existence of a single SU receiver in Chapter 7, the SNR at the SU 
receiver represents the most meaningful metric to optimise. The system model assumed that the SU receiver was located at a large distance away from the PU transmitters, hence, PU transmit powers were sufficiently attenuated by distance to be ignored at the SU receiver. A drawback of this approach is that the solution heavily relies on this assumption and no margin has been allocated to deal with any interference. The performance is expected to degrade in the presence of interference. Hence, the solution could be improved by taking into account the interference from the PUs.

Despite some of the shortcomings identified above, we believe that the research presented in this thesis provides valuable insights into robust methods of spectrum sharing for cognitive radio systems and lays a solid groundwork for future research in this area.

\subsection{Suggestions for Future Research}

As discussed above, the robust beamformers proposed in Chapter 6 could be improved by taking into account CSI uncertainty of all links in the system. A significant contribution could be made by developing robust cooperative beamformers that consider CSI uncertainty of all links in the system.

A significant contribution could be made by developing distributed algorithms for solving the optimisation problems proposed in Chapters 5 and 6 .

To realise the full benefits of transmit beamforming, full CSIT is required. Unfortunately, this requirement is impractical in real systems. Limited feedback systems [144] in which the receivers send highly quantised CSI to the transmitter have been shown to provide benefits nearly identical to unrealisable perfect transmitter channel knowledge systems when they are judiciously designed. A significant contribution could be made by extending the stochastic robust optimisation methods for imperfect CSI (Chapters 6 and 7) to limited feedback systems, both conventional and CR systems. Specifically, if one is able to compute the PDF of the error between un-quantised channel coefficients and the codebook entries, then the robust stochastic methods developed in this thesis could be directly applied.

Finally, base-station cooperation or coordinated downlink beamforming (CBF) [258] in cellular networks has received much recent attention as a means to raise overall data rate capacity. This idea could be extended to cellular CR systems. Significant contributions could be made to the problem of robust optimum user allocation under channel uncertainty and to methods that reduce the CSI exchange requirements between base-stations. 



\section{Bibliography}

[1] I. F Akyildiz, W. Y. Lee, M. C. Vuran, and S. Mohanty. NeXt generation/dynamic spectrum access/cognitive radio wireless networks: A survey. Comput. Netw., 50(13):2127-2159, 2006.

[2] M. Alayesh and N. Ghani. Game-theoretic approach for primary-secondary user power control under fast flat fading channels. IEEE Commun. Lett., 15(5):491-493, May 2011.

[3] M. Andersin, Z. Rosberg, and J. Zander. Gradual removals in cellular PCS with constrained power control and noise. Wireless Netw., 2(1):27-43, 1996.

[4] K. M. Anstreicher. Progress in interior point algorithms since 1984. SIAM News, 22:12-14, March 1989.

[5] S. Ariyavisitakul. SIR-based power control in a CDMA system. In Proc. IEEE GLOBECOM, pages 868-873, 1992.

[6] V. Asghari and S. Aissa. Performance of cooperative spectrum-sharing systems with amplify-and-forward relaying. IEEE Trans. Wireless Commun., 11(4):1295 -1300, Apr. 2012.

[7] D. Astély and B. Ottersten. The effects of local scattering on direction of arrival estimation with MUSIC. IEEE Trans. Signal Process., 47(12):3220$3234,1999$.

[8] M. Avriel, editor. Advances in Geometric Programming, volume 21 of Mathematical Concepts and Methods in Science and Engineering. Plenum Press, 1980.

[9] E. Axell and E. G. Larsson. A Bayesian approach to spectrum sensing, denoising and anomaly detection. In Proc. ICASSP, pages 2333-2336, 2009.

[10] E. Axell and E. G. Larsson. Spectrum sensing of orthogonal space-time block coded signals with multiple receive antennas. In Proc. ICASSP, pages 31103113, 2010. 
[11] E. Axell and E. G. Larsson. Optimal and sub-optimal spectrum sensing of OFDM signals in known and unknown noise variance. IEEE J. Sel. Areas Commun., 29(2):290-304, 2011.

[12] E. Axell and E. G. Larsson. A unified framework for GLRT-based spectrum sensing of signals with covariance matrices with known eigenvalue multiplicities. In Proc. ICASSP, pages 2956-2959, 2011.

[13] E. Axell, G. Leus, E. G. Larsson, and H. V. Poor. Spectrum sensing for cognitive radio: State-of-the-art and recent advances. IEEE Signal Process. Mag., 29(3):101-116, 2012.

[14] N. Bambos. Toward power-sensitive network architectures in wireless communications. IEEE Pers. Commun. Mag., 5(3):50-59, 1998.

[15] D. A. Bayer and J. C. Lagarias. The nonlinear geometry of linear programming, Part I : Affine and projective scaling trajectories. Transactions of the American Mathematical Society, 314(2):499-526, 1989.

[16] D. A. Bayer and J. C. Lagarias. The nonlinear geometry of linear programming, Part II : Legendre transform coordinates. Transactions of the American Mathematical Society, 314(2):527-581, 1989.

[17] A. Beck and M. Teboulle. Gradient-based algorithms with applications to signal-recovery problems. In D. P. Palomar and Y. C. Eldar, editors, Convex Optimization in Signal Processing and Communications, pages 42-88. Cambridge University Press, 2010.

[18] C. S. Beightler and D. T. Philips. Applied Geometric Programming. Wiley, 1976.

[19] A. Ben-Tal and A. Nemirovski. Robust convex optimization. Math. Oper. Res., 23(4):769-805, 1998.

[20] A. Ben-Tal and A. Nemirovski. Robust solutions of uncertain linear programs. Oper. Res. Lett., 25(1):1-13, 1999.

[21] A. Ben-Tal and A. Nemirovski. Robust solutions of linear programming problems contaminated with uncertain data. Math. Program., 88(3):411-424, 2000 .

[22] A. Ben-Tal and A. Nemirovski. Robust optimization-methodology and applications. Math. Program., 92(3):453-480, 2002.

[23] A. Ben-Tal, A. Nemirovski, and C. Roos. Robust solutions of uncertain quadratic and conic-quadratic problems. SIAM J. Optim., 13(2):535-560, 2002. 
[24] M. Bengtsson and B. Ottersten. Optimal downlink beamforming using semidefinite optimization. In Proc. Annual Allerton Conf. on Commun., Control, Comp., volume 37, pages 987-996, 1999.

[25] M. Bengtsson and B. Ottersten. Optimal and suboptimal transmit beamforming. In L. C. Godara, editor, Handbook on Antennas in Wireless Communications. CRC, 2002.

[26] D. Bertsimas, D. B. Brown, and C. Caramanis. Theory and applications of robust optimization. SIAM Review, 53(3):464-501, 2011.

[27] O. Besson and P. Stoica. Decoupled estimation of DOA and angular spread for a spatially distributed source. IEEE Trans. Signal Process., 48(7):1872$1882,2000$.

[28] J. R. Birge and F. Louveaux. Introduction to Stochastic Programming. Springer, New York, 1997.

[29] S. Boyd and L. Vandenberghe. Convex Optimization. Cambridge University Press, 2009.

[30] S. P. Boyd, Ghaoui E. L., E. Feron, and V. Balakrishnan. Linear matrix inequalities in system and control theory, volume 15. SIAM, 1994.

[31] L. E. Brennan, J. Mallett, and I. S. Reed. Adaptive arrays in airborne MTI radar. IEEE Trans. Antennas Propag., 24(5):607-615, 1976.

[32] V. Brik, E. Rozner, S. Banerjee, and P. Bahl. DSAP: a protocol for coordinated spectrum access. In Proc. IEEE DySPAN, volume 5, pages 611-614, 2005 .

[33] G. W. Brown and T. C. Koopmans. Computational suggestions for maximizing a linear function subject to linear inequalities. In T. C. Koopmans, editor, Activity Analysis of Production and Allocation, pages 377-380. John Wiley \& Sons, New York, NY, USA, 1951.

[34] D. Cabric, S. Mu. Mishra, and R. W. Brodersen. Implementation issues in spectrum sensing for cognitive radios. In Proc. Asilomar Conf Signals, Syst. Comput., volume 1, pages 772-776, 2004.

[35] J. Capon. High-resolution frequency-wavenumber spectrum analysis. Proc. IEEE, 57(8):1408-1418, 1969.

[36] B. D. Carlson. Covariance matrix estimation errors and diagonal loading in adaptive arrays. IEEE Trans. Aerosp. Electron. Syst., 24(4):397-401, 1988. 
[37] C. W. Caroll. The created response surface technique for optimizing nonlinear restrained systems. Oper. Res., 9(2):169-184, 1961.

[38] B. K. Chalise, S. Shahbazpanahi, A. Czylwik, and A. B. Gershman. Robust downlink beamforming based on outage probability specifications. IEEE Trans. Wireless Commun., 6(10):3498-3503, Oct. 2007.

[39] B. K. Chalise and L. Vandendorpe. MIMO relay design for multipoint-tomultipoint communications with imperfect channel state information. IEEE Trans. Signal Process., 57(7):2785-2796, 2009.

[40] L. Chang and C. C. Yeh. Performance of DMI and eigenspace-based beamformers. IEEE Trans. Antennas Propag., 40(11):1336-1347, 1992.

[41] T.-H. Chang, Z.-Q. Luo, and C.-Y. Chi. Approximation bounds for semidefinite relaxation of max-min-fair multicast transmit beamforming problem. IEEE Trans. Signal Process., 56(8):3932-3943, 2008.

[42] A. Charnes and W. W. Cooper. Programming with linear fractional functionals. Naval Research logistics quarterly, 9(3-4):181-186, 1962.

[43] S. Chaudhari, V. Koivunen, and H. V. Poor. Autocorrelation-based decentralized sequential detection of OFDM signals in cognitive radios. IEEE Trans. Signal Process., 57(7):2690-2700, 2009.

[44] H. S. Chen, W. Gao, and D. G. Daut. Spectrum sensing using cyclostationary properties and application to IEEE 802.22 WRAN. In Proc. IEEE GLOBECOM, pages 3133-3138, 2007.

[45] Y. Chen, G. Yu, , Z. Zhang, H. Chen, and P. Qiu. On cognitive radio networks with opportunistic power control strategies in fading channels. IEEE Trans. Wireless Commun., 7(7):2752-2761, Jul. 2008.

[46] M. Chiang, C. W. Tan, D. Palomar, D. O’Neill, and D. Julian. Power control by geometric programming. IEEE Trans. Wireless Commun., 6(7):26402651, Jul. 2007.

[47] Cisco. Cisco Visual Networking Index: Global Mobile Data Traffic Forecast Update, 2013-2018. Technical report, Feb. 2014.

[48] Federal Communications Commission. Spectrum Policy Task force. Technical report, Rep. ET Docket no. 02-135, Nov. 2002.

[49] Federal Communications Commission. Mobile broadband: The benefits of additional spectrum. Technical report, Oct. 2010. 
[50] M. H. M. Costa. On the Gaussian interference channel. IEEE Trans. Info. Theory, 31(5):607-615, 1985.

[51] R. Couillet and M. Debbah. A Bayesian framework for collaborative multisource signal sensing. IEEE Trans. Signal Process., 58(10):5186-5195, 2010.

[52] A. J. Coulson. Blind detection of wideband interference for cognitive radio applications. EURASIP J. Advances Signal Process., 2009:8, 2009.

[53] A. J. Coulson. Spectrum sensing using hidden Markov modeling. In Proc. IEEE ICC, pages 1-6, 2009.

[54] A. J. Coulson. Blind spectrum sensing using Bayesian sequential testing with dynamic update. In Proc. IEEE ICC, pages 1-6, 2011.

[55] H. Cox. Resolving power and sensitivity to mismatch of optimum array processors. J. Acoust. Soc. Amer., 54(3):771-785, 2005.

[56] H. Cox, R. M. Zeskind, and M. M. Owen. Robust adaptive beamforming. IEEE Trans. Acoust., Speech, Signal Process., 35(10):1365-1376, 1987.

[57] E. Dahlman, H. Ekstrom, A Furuskar, Y. Jading, J. Karlsson, M. Lundevall, and S. Parkvall. The 3G Long-Term Evolution - Radio Interface Concepts and Performance Evaluation. In Proc. IEEE VTC, volume 1, pages 137-141, May 2006.

[58] A. V. Dandawate and G. B. Giannakis. Statistical tests for presence of cyclostationarity. IEEE Trans. Signal Process., 42(9):2355-2369, 1994.

[59] G. B. Dantzig. Linear Programming and Extensions. Princeton University Press, 1963.

[60] D. Datla, A. M. Wyglinski, and G. J. Minden. A spectrum surveying framework for dynamic spectrum access networks. IEEE Trans. Veh. Technol., 58(8):4158-4168, 2009.

[61] A. De Maio, S. De Nicola, Y. Huang, S. Zhang, and A. Farina. Code design to optimize radar detection performance under accuracy and similarity constraints. IEEE Trans. Signal Process., 56(11):5618-5629, 2008.

[62] D. den Hertog. Interior Point Approach to Linear, Quadratic and Convex Programming. Kluwer, Norwell, MA, 1993.

[63] F. F. Digham, M. S. Alouini, and M. K. Simon. On the energy detection of unknown signals over fading channels. IEEE Trans. Commun., 55(1):21-24, 2007. 
[64] I. Dikin. Iterative solution of problems of linear and quadratic programming. Soviet Math. Dokl., 8:674-675, 1967.

[65] P. A. Dmochowski, H. A. Suraweera, P. J. Smith, and M. Shafi. Impact of channel knowledge on cognitive radio system capacity. In Proc. IEEE VTC2010-Fall, pages 1-5, Sept. 2010.

[66] L. El Ghaoui and H. Lebret. Robust solutions to least-squares problems with uncertain data. SIAM J. Matrix Anal. Appl., 18(4):1035-1064, 1997.

[67] L. El Ghaoui, F. Oustry, and H. Lebret. Robust solutions to uncertain semidefinite programs. SIAM J. Optim., 9(1):33-52, 1998.

[68] A. El-Keyi and B. Champagne. Collaborative uplink transmit beamforming with robustness against channel estimation errors. IEEE Trans. Veh. Technol., 58(1):126-139, 2009.

[69] A. El-Keyi, T. Kirubarajan, and A.B. Gershman. Wideband robust beamforming based on worst-case performance optimization. In IEEE 13th Workshop on Statistical Signal Process., pages 265 -270, Jul. 2005.

[70] M. H. Er and A. Cantoni. An alternative formulation for an optimum beamformer with robustness capability. IEE Proc. Commun., Radar, Signal Process., 132(6):447-460, 1985.

[71] D. D. Feldman and L. J. Griffiths. A projection approach for robust adaptive beamforming. IEEE Trans. Signal Process., 42(4):867-876, 1994.

[72] A. Fiacco and G. McCormick. Nonlinear programming: Sequential unconstrained minimization techniques. Wiley, New York, 1968. SIAM Classics in Applied Mathematics Series, 1990, reprint.

[73] C. Fischione, M. D'Angelo, and M. Butussi. Utility maximization via power and rate allocation with outage constraints in Nakagami-lognormal channels. IEEE Trans. Wireless Commun., 10(4):1108-1120, 2011.

[74] J. P. Fishburn and A. E. Dunlop. TILOS: A posynomial programming approach to transistor sizing. In The Best of ICCAD, pages 295-302. Springer, 2003.

[75] G. Foschini and Z. Miljanic. A simple distributed autonomous power control algorithm and its convergence. IEEE Trans. Veh. Technol., 42(4):641-646, Nov. 1993.

[76] A. L. Fradkov and V. A. Yakubovich. The S-procedure and duality relations in nonconvex problems of quadratic programming. Vestn. LGU, Ser. Mat., Mekh., Astron, (1):101-109, 1979. 
[77] K. R. Frisch. Principles of linear programming - With a particular reference to double gradient form of the logarithmic potential method. Memorandum, Institute of Economics, University of Oslo, Oslo, Norway, Oct. 1954.

[78] K. R. Frisch. The logarithmic potential method for convex programming. Unpublished manuscript, Institute of Economics, University of Oslo, Oslo, Norway, May 1955.

[79] K. R. Frisch. The multiplex method for linear programming. Sankhya, 18:329-362, 1957.

[80] W. A. Gardner. Exploitation of spectral redundancy in cyclostationary signals. IEEE Signal Process. Mag., 8(2):14-36, 1991.

[81] A. B. Gershman, E. Nemeth, and J. F. Böhme. Experimental performance of adaptive beamforming in a sonar environment with a towed array and moving interfering sources. IEEE Trans. Signal Process., 48(1):246-250, 2000.

[82] A. B. Gershman, N. D. Sidiropoulos, S. Shahbazpanahi, M. Bengtsson, and B. Ottersten. Convex optimization based beamforming. IEEE Signal Process. Mag., 27(3):62-75, May 2010.

[83] A. B. Gershman, V. I. Turchin, and V. A. Zverev. Experimental results of localization of moving underwater signal by adaptive beamforming. IEEE Trans. Signal Process., 43(10):2249-2257, 1995.

[84] A.B. Gershman, Z.-Q. Luo, S. Shahbazpanahi, and S.A. Vorobyov. Robust adaptive beamforming using worst-case performance optimization. In 37th Asilomar Conf. on Signals, Systems and Computers, volume 2, pages 13531357, Nov. 2003.

[85] A. Ghasemi and E. S. Sousa. Fundamental limits of spectrum-sharing in fading environments. IEEE Trans. Wireless Commun., 6:649-658, Feb. 2007.

[86] M. Ghozzi, F. Marx, M. Dohler, and J. Palicot. Cyclostationarity-based test for detection of vacant frequency bands. In Proc. IEEE Int. Conf. Cognitive Radio Oriented Wireless Netw. Commun., pages 1-5, 2006.

[87] L. Godara. The effect of phase-shifter errors on the performance of an antenna-array beamformer. IEEE J. Ocean. Eng., 10(3):278-284, 1985.

[88] L. C. Godara. Application of antenna arrays to mobile communications. II. Beam-forming and direction-of-arrival considerations. Proc. IEEE, 85(8):1195-1245, 1997. 
[89] M. X. Goemans and D. P. Williamson. Improved approximation algorithms for maximum cut and satisfiability problem using semi-definite programming. J. ACM, 42(6):1115-1145, 1995.

[90] A. Goldsmith, S. A. Jafar, I. Maric, and S. Srinivasa. Breaking spectrum gridlock with cognitive radios: An information theoretic perspective. Proc. IEEE, 97(5):894-914, 2009.

[91] C.C. Gonzaga. Path-following methods for linear programming. SIAM Review, 34:167-224, 1992.

[92] M. Grant and S. Boyd. Cvx: Matlab software for disciplined convex programming. Available: http://cvxr.com/cvx.

[93] L. J. Griffiths and C. W. Jim. An alternative approach to linearly constrained adaptive beamforming. IEEE Trans. Antennas Propag., 30(1):27-34, 1982.

[94] IEEE 802.22 Working Group. IEEE Standard for Cognitive Wireless Regional Area Networks (RAN) for Operation in TV Bands. IEEE Std. 802.22-2011, 2011.

[95] A. K. Gupta and D. K. Nagar. Matrix Variate Distributions. Chapman \& Hall/CRC, 1999.

[96] K. Hamdi, K. Zarifi, K. B. Letaief, and A. Ghrayeb. Beamforming in relayassisted cognitive radio systems: A convex optimization approach. In Proc. IEEE ICC 2011, pages 1-5, Jun. 2011.

[97] N. Han, S. Shon, J. H. Chung, and J. M. Kim. Spectral correlation based signal detection method for spectrum sensing in IEEE 802.22 WRAN systems. In Proc. ICACT, volume 3, pages 6-pp, 2006.

[98] K. Harmanci, J. Tabrikian, and J. L. Krolik. Relationships between adaptive minimum variance beamforming and optimal source localization. IEEE Trans. Signal Process., 48(1):1-12, 2000.

[99] V. Havary-Nassab, S. Shahbazpanahi, A. Grami, and Z. Luo. Distributed beamforming for relay networks based on second-order statistics of the channel state information. IEEE Trans. Signal Process., 56(9):4306-4316, Sept. 2008 .

[100] V. Havary-Nassab, S. Shahbazpanahi, A. Grami, and Z.-Q. Luo. Network beamforming based on second order statistics of the channel state information. In IEEE Int. Conf. Acoustics, Speech and Signal Process., pages 2605-2608, 2008. 
[101] S. Haykin. Digital Communications. John Wiley \& Sons, Inc, 1988.

[102] S. Haykin. Cognitive radio: brain-empowered wireless communications. IEEE J. Sel. Areas Commun., 23(2):201-220, 2005.

[103] S. Haykin, D. J. Thomson, and J. H. Reed. Spectrum sensing for cognitive radio. Proc. IEEE, 97(5):849-877, 2009.

[104] S. S. Haykin, J. Litva, and T. J. Shepherd. Radar array processing, volume 25. Springer, 1993.

[105] C. Helmberg. Semidefinite programming. European J. Oper. Res., 137(3):461-482, Mar. 2002.

[106] Y. J Hong, C. C. Yeh, and D. R. Ucci. The effect of a finite-distance signal source on a far-field steering applebaum array-two dimensional array case. IEEE Trans. Antennas Propag., 36(4):468-475, 1988.

[107] K. Hossain and B. Champagne. Wideband spectrum sensing for cognitive radios with correlated subband occupancy. IEEE Signal Process. Lett., 18(1):35-38, 2011.

[108] N. Hoven, R. Tandra, and A. Sahai. Some fundamental limits on cognitive radio. Wireless Foundations EECS, Univ. of California, Berkeley, 2005.

[109] J. Huang, R. A. Berry, and M. L. Honig. Spectrum sharing with distributed interference compensation. In NProc. IEEE DySPAN, pages 88-93, 2005.

[110] P. Huard. Resolution of mathematical programming with nonlinear constraints by the method of centers. In J. Abadie, editor, Nonlinear Programming, pages 207-219. 1967.

[111] M. C. Vuran I.F. Akyildiz, W.-Y. Lee and S. Mohanty. Next generation/dynamic spectrum access/cognitive radio wireless networks: a survey. Computer Networks, 50:2127-2159, 2006.

[112] M. H. Islam, C. L. Koh, S. W. Oh, X. Qing, Y. Y. Lai, C. Wang, Y. C. Liang, B. E. Toh, F. Chin, G. L. Tan, and W. Toh. Spectrum survey in Singapore: Occupancy measurements and analyses. In Proc. 3rd Int. Conf. CROWNCOM, pages 1-7, 2008.

[113] N. K. Jablon. Adaptive beamforming with the generalized sidelobe canceller in the presence of array imperfections. IEEE Trans. Antennas Propag., 34(8):996-1012, 1986. 
[114] S. A. Jafar and S. Srinivasa. Capacity limits of cognitive radio with distributed and dynamic spectral activity. IEEE J. Sel. Areas Commun., 25:529537, Apr. 2007.

[115] Q. Jin, D. Yuan, and Z. Guan. Distributed geometric-programming-based power control in cellular cognitive radio networks. In Proc. VTC 2009, pages 1-5, Apr. 2009.

[116] Y. Kameda and J. Ohga. Adaptive microphone-array system for noise reduction. IEEE Trans. Acoust., Speech, Signal Process., 34(6):1391-1400, 1986.

[117] S. Kandukuri and S. Boyd. Optimal power control in interference limited fading wireless channels with outage probability specifications. IEEE Trans. Wireless Commun., 1(1):46-55, Jan. 2002.

[118] X. Kang, Y.-C. Liang, A. Nallanathan, H. K. Garg, and R. Zhang. Optimal power allocation for fading channels in cognitive radio networks: Ergodic capacity and outage capacity. IEEE Trans. Wireless Commun., 8:940-950, Feb. 2009.

[119] E. Karipidis, N. D. Sidiropoulos, and Z.-Q. Luo. Far-field multicast beamforming for uniform linear antenna arrays. IEEE Trans. Signal Process., 55(10):4916-4927, 2007.

[120] E. Karipidis, N. D. Sidiropoulos, and Z.-Q. Luo. Quality of service and maxmin fair transmit beamforming to multiple cochannel multicast groups. IEEE Trans. Signal Process., 56(3):1268-1279, 2008.

[121] N. K. Karmarkar. A new polynomial time algorithm for linear programming. In Proc. 16th Annual ACM Symposium on Theory of Computing, pages 302$311,1984$.

[122] N. K. Karmarkar. A new polynomial time algorithm for linear programming. Combinatorica, 4:373-395, 1984.

[123] N. K. Karmarkar. Some comments on the significance of the new polynomialtime algorithm for linear programming. Technical report, AT \& T Bell Laboratories, Murray Hill, NJ 07974, USA, 1984.

[124] N. K. Karmarkar. Why is the new algorithm better than simplex method and ellipsoid method? Extended Abstract, AT \& T Bell Laboratories, Murray Hill, NJ 07974, USA, 1984/85.

[125] S. M. Kay. Fundamentals of statistical signal processing, Vol. II: Detection Theory. Prentice Hall, Upper Saddle River, NJ, USA, 1998. 
[126] N. Khambekar, L. Dong, and V. Chaudhary. Utilizing OFDM guard interval for spectrum sensing. In Proc. IEEE WCNC, pages 38-42, 2007.

[127] J. W. Kim and C. K. Un. An adaptive array robust to beam pointing error. IEEE Trans. Signal Process., 40(6):1582-1584, 1992.

[128] V. Klee and G. J. Minty. How good is the simplex algorithm? In Proc. of the Third Symposium on Inequalities, pages 159-175, Sept. 1969.

[129] M. Kojima, N. Megiddo, T. Noma, and A. Yoshise. A unified approach to interior point algorithms for linear complementarity problems. Lecture Notes in Computer Science, Springer-Verlag, New York, Berlin, 1991.

[130] P. Kolodzy et al. Next generation communications: Kickoff meeting. In Proc. DARPA, volume 10, 2001.

[131] J. L. Krolik. The performance of matched-field beamformers with Mediterranean vertical array data. IEEE Trans. Signal Process., 44(10):2605-2611, 1996.

[132] J. C. Lagarias. The nonlinear geometry of linear programming, Part III : Projective legrende transform coordinates and Hilbert geometry. Transactions of the American Mathematical Society, 320:193225, 1990.

[133] T.-H. Lee, J.-C. Lin, and Y. T. Su. Downlink power control algorithms for cellular radio systems. IEEE Trans. Veh. Technol., 44(1):89-94, 1995.

[134] Z. Lei and F. Chin. OFDM signal sensing for cognitive radios. In Proc. IEEE PIMRC, pages $1-5,2008$.

[135] D. Li and X. Dai. Power control in cooperative cognitive radio networks by geometric programming. In Proc. APCC 2009, pages 118-121, Oct. 2009.

[136] J. Li, A. P. Petropulu, and H. V. Poor. Cooperative transmission for relay networks based on second-order statistics of channel state information. IEEE Trans. Signal Process., 59(3):1280-1291, 2011.

[137] J. Li, P. Stoica, and Z. Wang. On robust capon beamforming and diagonal loading. IEEE Trans. Signal Process., 51(7):1702-1715, 2003.

[138] Y. C. Liang, K. C. Chen, G. Y. Li, and P. Mahonen. Cognitive radio networking and communications: An overview. IEEE Trans. Veh. Technol., 60(7):3386-3407, 2011.

[139] J.-C. Lin, T.-H. Lee, and Y.-T. Su. Power control algorithm for cellular radio systems. Elec. Lett., 30(3):195-197, 1994. 
[140] J. Liu, W. Chen, Z. Cao, and Y. J. A. Zhang. Cooperative beamforming for cognitive radio networks: A cross-layer design. IEEE Trans. Commun., 60(5):1420-1431, May 2012.

[141] F. A. Lootsma. Numerical Methods for Nonlinear Optimization. Academic Press, London, UK, 1972.

[142] R. G. Lorenz and S. P. Boyd. Robust minimum variance beamforming. IEEE Trans. Signal Process., 53(5):1684-1696, 2005.

[143] R. G. Lorenz and S. P. Boyd. Robust minimum variance beamforming. In J. Li and P. Stoica, editors, Robust Adaptive Beamforming. John Wiley \& Sons, Inc, 2006.

[144] D. J. Love, R. W. Heath, V. K. N. Lau, D. Gesbert, B. D. Rao, and M. Andrews. An overview of limited feedback in wireless communication systems. IEEE J. Sel. Areas in Commun., 26(8):1341-1365, 2008.

[145] J. Lundén, V. Koivunen, A. Huttunen, and H. V. Poor. Spectrum sensing in cognitive radios based on multiple cyclic frequencies. In Proc. CROWNCOM, pages 37-43, 2007.

[146] Z. Luo and T. Chang. SDP relaxation of homogeneous quadratic optimization: approximation bounds and applications. In D. P. Palomar and Y. C. Eldar, editors, Convex Optimization in Signal Processing and Communications, pages 117-165. Cambridge University Press, 2010.

[147] Z. Luo, W. Ma, A. So, Y. Ye, and S. Zhang. Semidefinite relaxation of quadratic optimization problems. IEEE Signal Process. Mag., 27(3):20-34, May 2010.

[148] Z. Luo and W. Yu. An introduction to convex optimization for communications and signal processing. IEEE J. Sel. Areas Commun., 24(8):1426-1438, Aug. 2006.

[149] Z.-Q. Luo, X. Luo, and M. Kisialiou. An efficient quasi-maximum likelihood decoder for PSK signals. In Proc. IEEE Int. Conf. on Acoustics, Speech, and Signal Process., volume 6, pages VI-561-IV-4, 2003.

[150] J. Ma, G. Y. Li, and B. H. Juang. Signal processing in cognitive radio. Proc. IEEE, 97(5):805-823, 2009.

[151] L. Ma, X. Han, and C. C. Shen. Dynamic open spectrum sharing MAC protocol for wireless ad hoc networks. In Proc. IEEE DySPAN, pages 203$213,2005$. 
[152] W.-K. Ma, P.-C. Ching, and Z. Ding. Semidefinite relaxation based multiuser detection for M-ary PSK multiuser systems. IEEE Trans. Signal Process., 52(10):2862-2872, 2004.

[153] W.-K. Ma, T. N. Davidson, K. M. Wong, Z.-Q. Luo, and P.-C. Ching. Quasimaximum-likelihood multiuser detection using semi-definite relaxation with application to synchronous CDMA. IEEE Trans. Signal Process., 50(4):912922, 2002.

[154] W.-K. Ma, C.-C. Su, J. Jaldén, T.-H. Chang, and C.-Y. Chi. The equivalence of semidefinite relaxation MIMO detectors for higher-order QAM. IEEE J. Sel. Topics Signal Process., 3(6):1038-1052, 2009.

[155] K. Maeda, A. Benjebbour, T. Asai, T. Furuno, and T. Ohya. Recognition among OFDM-based systems utilizing cyclostationarity-inducing transmission. In Proc. IEEE DySPAN, pages 516-523, 2007.

[156] Z. Mao, X. Wang, and X. Wang. Semidefinite programming relaxation approach for multiuser detection of QAM signals. IEEE Trans. Wireless Commun., 6(12):4275-4279, 2007.

[157] A. M. Mathai and Serge B. Provost. Quadratic Forms In Random Variables, Theory and Applications. Marcel Dekker, INC., 1992.

[158] M. McHenry. Frequency agile spectrum access technologies. In FCC Workshop Cogn. Radios, volume 19, 2003.

[159] M. A. McHenry. NSF spectrum occupancy measurements project summary. Shared spectrum company report, 2005.

[160] N. Megiddo. Progress in Mathematical Programming : Interior Point and Related Methods. Springer-Verlag, New York, NY, USA, 1993.

[161] A. Megretski and S. Treil. Power distribution inequalities in optimization and robustness of uncertain systems. J. Math. Syst. Estim. Control, 3(3):301-319, 1993.

[162] S. M. Mishra, S. ten Brink, R. Mahadevappa, and R. W. Brodersen. Cognitive technology for ultra-wideband/WiMax coexistence. In Proc. DySPAN, pages 179-186, 2007.

[163] J. Mitola. Cognitive radio: An integrated agent architecture for software defined radio. PhD thesis, KTH Royal Institute of Technology, Sweden, May 2000 . 
[164] J. Mitola and G. Q. Maguire Jr. Cognitive radio: making software radios more personal. IEEE Person. Commun., 6(4):13-18, 1999.

[165] D. Mitra. An asynchronous distributed algorithm for power control in cellular radio systems. In Wireless and Mobile Commun., pages 177-186. Springer, 1994.

[166] A. Mobasher, M. Taherzadeh, R. Sotirov, and A. K. Khandani. A nearmaximum-likelihood decoding algorithm for MIMO systems based on semidefinite programming. IEEE Trans. Inf. Theory, 53(11):3869-3886, 2007.

[167] C. L. Monma. Successful implementations of interior algorithms. SIAM News, 22(2):14-16, March 1989.

[168] R. A. Monzingo and T. W. Miller. Introduction to Adaptive Arrays. Wiley, New York, 1980.

[169] P. Mousavi, L. Shafai, B. Veidt, and P. Dewdney. Feed-reflector design for large adaptive reflector antenna (LAR). IEEE Trans Antennas Propag., 49(8):1142-1154, 2001.

[170] L. Musavian and S. Aissa. Fundamental capacity limits of spectrum-sharing channels with imperfect feedback. In Proc. IEEE GLOBECOM 200\%, pages 1385-1389, Nov. 2007.

[171] A. Nemirovski. Lecture notes: Interior point polynomial time methods in convex programming. Available: http://www2.isye.gatech.edu/ nemirovs/ Lect_IPM.pdf, 2004.

[172] Y. Nesterov and A. Nemirovski. A general approach to polynomial-time algorithms design for convex programming. Technical report, Centr. Econ. and Math. Inst., USSR Acad. Sci., Moscow, USSR, 1988.

[173] Y. Nesterov and A. Nemirovski. Self-concordant functions and polynomial time methods in convex programming. Technical report, Centr. Econ. and Math. Inst., USSR Acad. Sci., Moscow, USSR, 1990.

[174] Y. Nesterov and A. Nemirovski. Conic formulation of a convex programming problem and duality. Technical report, Centr. Econ. and Math. Inst., USSR Acad. Sci., Moscow, USSR, 1991.

[175] Y. Nesterov and A. Nemirovski. Interior Point Polynomial Methods in Convex Programming. SIAM Press, 1994.

[176] R. W. Nettleton and H. Alavi. Power control for a spread spectrum cellular mobile radio system. In Proc. IEEE VTC, volume 33, pages 242-246, 1983. 
[177] G. R. Parisot. Approximate numerical solution of linear programming problems by an application of the logarithmic programming. Revue Francaise Recherche Operationelle, 20:227-259, 1961.

[178] H. V. Poor. An Introduction to Signal Detection and Estimation. Springer, 1994.

[179] J. G. Proakis. Digital Communications. McGraw-Hill, 4th edition, 2001.

[180] P. Qihang, Z. Kun, W. Jun, and L. Shaoqian. A distributed spectrum sensing scheme based on credibility and evidence theory in cognitive radio context. In Proc. IEEE PIMRC, pages 1-5, 2006.

[181] Z. Qing, T. Liang, and A. Swami. Decentralized cognitive MAC for dynamic spectrum access. In Proc. IEEE DySPAN, pages 224-232, 2005.

[182] T. S. Rappaport. Smart Antennas: Adaptive Arrays, Algorithms, 86 Wireless Position Location. IEEE Press, 1998.

[183] F. Rashid-Farrokhi, K. J. R. Liu, and L. Tassiulas. Transmit beamforming and power control for cellular wireless systems. IEEE J. Sel. Areas Commun., 16(8):1437-1450, 1998.

[184] F. Rashid-Farrokhi, K. J. R. Liu, and L. Tassiulas. Transmit beamforming and power control for cellular wireless systems. IEEE J. Sel. Areas Commun., 16(8):1437 -1450, Oct. 1998.

[185] F. Rashid-Farrokhi, L. Tassiulas, and K. J. R. Liu. Joint optimal power control and beamforming in wireless networks using antenna arrays. IEEE Trans. Commun., 46(10):1313 -1324, Oct. 1998.

[186] M. Rasti, A. R. Sharafat, and J. Zander. Pareto and energy-efficient distributed power control with feasibility check in wireless networks. IEEE Trans. Inf. Theory, 57(1):245-255, 2011.

[187] I. S. Reed, J. D. Mallett, and L. E. Brennan. Rapid convergence rate in adaptive arrays. IEEE Trans. Aerosp. Electron. Syst., 10(6):853-863, 1974.

[188] W. Ren, Q. Zhao, and A. Swami. Power control in cognitive radio networks: How to cross a multi-lane highway. IEEE J. Sel. Areas Commun., 27(7):12831296, Sept. 2009.

[189] J. Renegar. A polynomial-time algorithm, based on Newtons method, for linear programming. Math. Program., 40:59-93, 1988. 
[190] J. Ringelstein, A. B. Gershman, and J. F. Böhme. Direction finding in random inhomogeneous media in the presence of multiplicative noise. IEEE Signal Process. Lett., 7(10):269-272, 2000.

[191] W. Roh and A. Paulraj. MIMO channel capacity for the distributed antenna. In Proc. VTC 2002-Fall, volume 2, pages 706-709, 2002.

[192] S. M. Ross. An Introduction to Mathematical Finance: Options and Other Topics. Cambridge University Press, 1999.

[193] A. Ruszczyński and A. Shapiro, editors. Stochastic Programming, volume 10 of Handbooks in Operations Research and Management Science. Elsevier, 2003.

[194] A. Sahai and D. Cabric. Spectrum sensing: fundamental limits and practical challenges. In Proc. IEEE DySPAN, 2005.

[195] S. S. Sapatnekar, V. B. Rao, P. M. Vaidya, and S.-M. Kang. An exact solution to the transistor sizing problem for CMOS circuits using convex optimization. Computer-Aided Design of Integrated Circuits and Systems, IEEE Transactions on, 12(11):1621-1634, 1993.

[196] C. U. Saraydar, N. B. Mandayam, and D. Goodman. Pricing and power control in a multicell wireless data network. IEEE J. Sel. Areas Commun., 19(10):1883-1892, 2001.

[197] H. Sato. The capacity of the Gaussian interference channel under strong interference (corresp.). IEEE Trans. Info. Theory, 27(6):786-788, 1981.

[198] L. L. Scharf. Statistical Signal Processing: Detection, Estimation, and Time Series Analysis. Addison-Wesley, Reading, MA, 1991.

[199] M. Schubert and H. Boche. Solution of the multiuser downlink beamforming problem with individual SINR constraints. IEEE Trans. Veh. Techol., 53(1):18 - 28, Jan. 2004.

[200] K. Sekihara, S. S. Nagarajan, D. Poeppel, and A. Marantz. Performance of an MEG adaptive-beamformer technique in the presence of correlated neural activities: effects on signal intensity and time-course estimates. IEEE Trans. Biomed. Eng., 49(12):1534-1546, 2002.

[201] A. Sendonaris, E. Erkip, and B. Aazhang. User cooperation diversity. Part I. System description. IEEE Trans. Commun., 51(11):1927-1938, Nov. 2003.

[202] A. Sendonaris, E. Erkip, and B. Aazhang. User cooperation diversity. Part II. Implementation aspects and performance analysis. IEEE Trans. Commun., 51(11):1939-1948, Nov. 2003. 
[203] P. Setoodeh and S. Haykin. Robust transmit power control for cognitive radio. Proc. IEEE, 97(5):915-939, May 2009.

[204] K. Seung-Jean, A. Magnani, A. Mutapcic, S.P. Boyd, and Zhi-Quan Luo. Robust Beamforming via Worst-Case SINR Maximization. IEEE Trans. Signal Process., 56(4):1539 -1547, Apr. 2008.

[205] S. Shahbazpanahi, A.B. Gershman, Zhi-Quan Luo, and Kon Max Wong. Robust adaptive beamforming for general-rank signal models. IEEE Trans. Signal Process., 51(9):2257 - 2269, Sep. 2003.

[206] N. S. Shankar, C. Cordeiro, and K. Challapali. Spectrum agile radios: utilization and sensing architectures. In IEEE DySPAN, pages 160-169, 2005.

[207] C. E. Shannon. The zero error capacity of a noisy channel. IRE Trans. Inf. Theory, 2(3):8-19, 1956.

[208] M. B. Shenouda and T. N. Davidson. On the design of linear transceivers for multiuser systems with channel uncertainty. IEEE J. Sel. Areas Commun., 26(6):1015-1024, 2008.

[209] M. B. Shenouda and T. N. Davidson. Probabilistically-constrained approaches to the design of the multiple antenna downlink. In 2008 42nd Asilomar Conf. Sig., Sys., Comp., pages 1120-1124, 2008.

[210] M. B. Shenouda and T. N. Davidson. Nonlinear and linear broadcasting with qos requirements: Tractable approaches for bounded channel uncertainties. IEEE Trans. Signal Process., 57(5):1936-1947, 2009.

[211] N. D. Sidiropoulos and T. N. Davidson. Broadcasting with channel state information. In Proc. IEEE SAM Workshop, pages 489-493, 2004.

[212] N. D. Sidiropoulos, T. N. Davidson, and Z.-Q. Luo. Transmit beamforming for physical-layer multicasting. IEEE Trans. Signal Process., 54(6):2239$2251,2006$.

[213] N. D. Sidiropoulos and Z.-Q. Luo. A semidefinite relaxation approach to MIMO detection for high-order QAM constellations. IEEE Signal Process. Lett., 13(9):525-528, 2006.

[214] P. Smith, P. Dmochowski, H. Suraweera, and M. Shafi. The effects of limited channel knowledge on cognitive radio system capacity. IEEE Trans. Veh. Technol., 62(2):927-933, Feb. 2013.

[215] P. Smith, T. King, L. Garth, and M. Dohler. A power scaling analysis of norm-based antenna selection techniques. IEEE Trans. on Wireless Commun., 7(8):3140-3149, Aug. 2008. 
[216] AM-C So. On the performance of semidefinite relaxation MIMO detectors for QAM constellations. In Proc. IEEE Int. Conf. on Acoustics, Speech and Signal Process., 2009, pages 2449-2452, 2009.

[217] A. Sonnenschein and P. M. Fishman. Radiometric detection of spreadspectrum signals in noise of uncertain power. IEEE Trans. Aerosp. Electron. Syst., 28(3):654-660, 1992.

[218] A. L. Soyster. Convex programming with set-inclusive constraints and applications to inexact linear programming. Oper. Res., 21(5):1154-1157, 1973.

[219] S. Srinivasa and S. A. Jafar. Soft sensing and optimal power control for cognitive radio. IEEE Trans. on Wireless Commun., 9(12):3638-3649, Dec. 2010.

[220] G. Staple and K. Werbach. The end of spectrum scarcity [spectrum allocation and utilization]. IEEE Spectrum, 41(3):48-52, 2004.

[221] P. Stoica, Z. Wang, and J. Li. Robust capon beamforming. IEEE Signal Process. Lett., 10(6):172-175, 2003.

[222] G. L. Stüber. Principles of Mobile Communication. Springer, 2011.

[223] C. W. Sung and W. S. Wong. Power control and rate management for wireless multimedia cdma systems. IEEE Trans. Commun., 49(7):1215-1226, 2001.

[224] H. A. Suraweera, P. J. Smith, and M. Shafi. Capacity limits and performance analysis of cognitive radio with imperfect channel knowledge. IEEE Trans. Veh. Technol., 59(4):1811-1822, 2010.

[225] H. A. Suraweera, P. J. Smith, M. Shafi, and M. Faulkner. Channel capacity limits of cognitive radio with imperfect channel knowledge. In Proc. IEEE GLOBECOM, pages 1-6, 2009.

[226] P. D. Sutton, J. Lotze, K. E. Nolan, and L. E. Doyle. Cyclostationary signature detection in multipath Rayleigh fading environments. In Proc. CROWNCOM, pages 408-413, 2007.

[227] P. D. Sutton, K. E. Nolan, and L. E. Doyle. Cyclostationary signatures in practical cognitive radio applications. IEEE J. Sel. Areas Commun., 26(1):13-24, 2008.

[228] P. H. Tan and L. K. Rasmussen. The application of semidefinite programming for detection in CDMA. IEEE J. Sel. Areas Commun., 19(8):1442-1449, 2001. 
[229] R. Tandra and A. Sahai. SNR walls for signal detection. IEEE J. Sel. Topics Signal Process., 2(1):4-17, 2008.

[230] H. Tang. Some physical layer issues of wide-band cognitive radio systems. In IEEE DySPAN, pages 151-159, 2005.

[231] L. Tang, H. Wang, and Q. Chen. Power allocation with min-max fairness for cognitive radio networks. In Proc. WCNIS 2010, pages 478-482, Jun. 2010.

[232] P. Ubaidulla and S. Aissa. Robust distributed cognitive relay beamforming. In Proc. VTC Spring 2012, pages 1-5, May 2012.

[233] H. Urkowitz. Energy detection of unknown deterministic signals. Proc. IEEE, 55(4):523-531, 1967.

[234] L. Vandenberghe and S. Boyd. Semidefinite programming. SIAM Review, 38(1):49-95, Mar. 1996.

[235] E. Visotsky and U. Madhow. Optimum beamforming using transmit antenna arrays. In Proc. VTC'99, volume 1, pages 851-856, 1999.

[236] S. A. Vorobyov, H. Chen, and A. B. Gershman. On the relationship between robust minimum variance beamformers with probabilistic and worst-case distortionless response constraints. IEEE Trans. Signal Process., 56(11):5719$5724,2008$.

[237] S. A. Vorobyov, A. B. Gershman, and Z.-Q. Luo. Robust adaptive beamforming using worst-case performance optimization via second-order cone programming. In Proc. Acoust., Speech, Signal Process. (ICASSP), volume 3, pages III-2901, 2002.

[238] S. A. Vorobyov, A. B. Gershman, and Z.-Q. Luo. Robust adaptive beamforming using worst-case performance optimization: A solution to the signal mismatch problem. IEEE Trans Signal Process., 51(2):313-324, 2003.

[239] N. Vucic and H. Boche. Robust QoS-constrained optimization of downlink multiuser MISO systems. IEEE Trans. Signal Process., 57(2):714-725, 2009.

[240] N. Vucic and H. Boche. A tractable method for chance-constrained power control in downlink multiuser MISO systems with channel uncertainty. IEEE Signal Process. Lett., 16(5):346-349, 2009.

[241] I. Wajid, Y. C. Eldar, and A. Gershman. Robust downlink beamforming using covariance channel state information. In Proc. Acoust., Speech, Signal Process. (ICASSP), pages 2285-2288, 2009. 
[242] B. Wang and K. J. R. Liu. Advances in cognitive radio networks: A survey. IEEE J. Sel. Topics Signal Process., 5(1):5-23, 2011.

[243] C.-X. Wang, X. Hong, H.-H. Chen, and J. Thompson. On capacity of cognitive radio networks with average interference power constraints. IEEE Trans. Wireless Commun., 8(4):1620-1625, Apr. 2009.

[244] P. Wang, J. Fang, N. Han, and H. Li. Multiantenna-assisted spectrum sensing for cognitive radio. IEEE Trans. Veh. Technol., 59(4):1791-1800, 2010.

[245] C. K. Wen, J. C. Chen, and P. Ting. Robust transmitter design for amplifyand-forward MIMO relay systems exploiting only channel statistics. Wireless Communications, IEEE Transactions on, 11(2):668 -682, Feb. 2012.

[246] A. Wiesel, Y. C. Eldar, and S. Shamai. Semidefinite relaxation for detection of 16-QAM signaling in MIMO channels. IEEE Signal Process. Lett., 12(9):653$656,2005$.

[247] A. Wiesel, Y.C. Eldar, and S. Shamai. Linear precoding via conic optimization for fixed MIMO receivers. IEEE Trans. Signal Process., 54(1):161 - 176, Jan. 2006.

[248] H. Wolkowicz, R. Saigal, and L. Vandenberghe, editors. Handbook of Semidefinite Programming. Kluwer's International Series. Kluwer Academic Publishers, 2003.

[249] V. A. Yakubovich. S-procedure in nonlinear control theory. Vestnik Leningrad Univ., 1:62-77, 1971.

[250] Y. Yang, C. Zhao, P. Zhou, and W. Xu. MIMO detection of 16-QAM signaling based on semidefinite relaxation. IEEE Signal Process. Lett., 14(11):797-800, 2007.

[251] Y. D. Yao and A. Sheikh. Outage probability analysis for microcell mobile radio systems with cochannel interferers in Rician/Rayleigh fading environment. Electron. Lett., 26(13):864-866, Jun. 1990.

[252] Y. D. Yao and A. Sheikh. Investigations into cochannel interference in microcellular mobile radio systems. IEEE Trans. Veh. Technol., 41(2):114-123, May 1992.

[253] R. D. Yates. A framework for uplink power control in cellular radio systems. IEEE J. Sel. Areas Commun., 13(7):1341-1347, 1995.

[254] R. D. Yates and C. Y. Huang. Integrated power control and base station assignment. IEEE Trans. Veh. Technol., 44(3):638-644, 1995. 
[255] Y.-S. Yeh and S. C. Schwartz. Outage probability in mobile telephony due to multiple log-normal interferers. IEEE Trans. Commun., 32(4):380-388, 1984 .

[256] T. Yucek and H. Arslan. Spectrum characterization for opportunistic cognitive radio systems. In Proc. IEEE Mil. Commun. Conf., pages 1-6, 2006.

[257] T. Yucek and H. Arslan. A survey of spectrum sensing algorithms for cognitive radio applications. IEEE Commun. Surveys Tuts., 11(1):116-130, 2009.

[258] R. Zakhour and S. V. Hanly. Base station cooperation on the downlink: Large system analysis. IEEE Trans. Info. Theory, 58(4):2079-2106, 2012.

[259] J. Zander. Performance of optimum transmitter power control in cellular radio systems. IEEE Trans. Veh. Technol., 41(1):57-62, 1992.

[260] S. A. Zekavat and X. Li. User-central wireless system: ultimate dynamic channel allocation. In Proc. IEEE DySPAN, pages 82-87, 2005.

[261] Y. Zeng, C. L. Koh, and Y. C. Liang. Maximum eigenvalue detection: theory and application. In Proc. IEEE ICC, pages 4160-4164, 2008.

[262] Y. Zeng and Y. C. Liang. Covariance based signal detections for cognitive radio. In Proc. IEEE DySPAN, pages 202-207, 2007.

[263] Y. Zeng and Y. C. Liang. Eigenvalue-based spectrum sensing algorithms for cognitive radio. IEEE Trans. Commun., 57(6):1784-1793, 2009.

[264] Y. Zeng and Y. C. Liang. Spectrum-sensing algorithms for cognitive radio based on statistical covariances. IEEE Trans. Veh. Technol., 58(4):1804-1815, 2009 .

[265] Y. Zeng, Y. C. Liang, A. T. Hoang, and R. Zhang. A review on spectrum sensing for cognitive radio: challenges and solutions. EURASIP J. Adv. Signal Process., 2010:2, 2010.

[266] J. Zhang and J. G. Andrews. Distributed antenna systems with randomness. IEEE Trans. Wireless Commun., 7(9):3636-3646, 2008.

[267] L. Zhang, Y. C. Liang, and Y. Xin. Joint beamforming and power allocation for multiple access channels in cognitive radio networks. IEEE J. Select. Areas Commun., 26(1):38 -51, Jan. 2008.

[268] L. Zhang, Y. C. Liang, Y. Xin, and H.V. Poor. Robust cognitive beamforming with partial channel state information. IEEE Trans. Wireless Commun., 8(8):4143 -4153, Aug. 2009. 
[269] R. Zhang, C. C. Chai, and Y. C. Liang. Joint beamforming and power control for multiantenna relay broadcast channel with QoS constraints. IEEE Trans. Signal Process., 57(2):726 -737, Feb. 2009.

[270] R. Zhang and Y. C. Liang. Exploiting multi-antennas for opportunistic spectrum sharing in cognitive radio networks. IEEE J. Select. Topics Signal Process., 2(1):88 -102, Feb. 2008.

[271] R. Zhang, Y. C. Liang, and S. Cui. Dynamic resource allocation in cognitive radio networks. IEEE Signal Process. Mag., 27(3):102-114, 2010.

[272] R. Zhang, Y. C. Liang, and S. Cui. Dynamic resource allocation in cognitive radio networks: A convex optimization perspective. arXiv, abs/1001.3187, 2010.

[273] R. Zhang, T. J. Lim, Y. C. Liang, and Y. Zeng. Multi-antenna based spectrum sensing for cognitive radios: A GLRT approach. IEEE Trans. Commun., 58(1):84-88, 2010.

[274] X. Zhang, D. P. Palomar, and B. Ottersten. Statistically robust design of linear MIMO transceivers. IEEE Trans. Signal Process., 56(8):3678-3689, 2008.

[275] Q. Zhao and B. M. Sadler. A survey of dynamic spectrum access. IEEE Signal Process. Mag., 24(3):79-89, 2007.

[276] G. Zheng, S. Ma, K-K. Wong, and T-S. Ng. Robust beamforming in cognitive radio. IEEE Trans. Wireless Commun., 9(2):570-576, 2010.

[277] G. Zheng, K. K. Wong, A. Paulraj, and B. Ottersten. Robust collaborativerelay beamforming. IEEE Trans. Signal Process., 57(8):3130-3143, Aug. 2009.

[278] H. Zheng and L. Cao. Device-centric spectrum management. In Proc. IEEE DySPAN, pages 56-65, 2005.

[279] Y. R. Zheng, R. A. Goubran, and M. El-Tanany. Experimental evaluation of a nested microphone array with adaptive noise cancellers. IEEE Trans. Instrum. Meas., 53(3):777-786, 2004.

[280] M. D. Zoltowski. On the performance analysis of the MVDR beamformer in the presence of correlated interference. IEEE Trans. Acoust., Speech, Signal Process., 36(6):945-947, 1988. 